\title{
Characterization of Industrial Process Waste Heat and Input Heat Streams
}
G. L. Wilfert
L. L. Fassbender
H. B. Huber
E. A. Griffin
R. E. Dodge
D. R. Brown
B. A. Garrett-Price
N. L. Moore

May 1984

Prepared for the U.S. Department of Energy under Contract DE-AC06-76RLO 1830

Pacific Northwest Laboratory Operated for the U.S. Department of Energy by Battelle Memorial Institute 


\title{
DISCLAIMER
}

This report was prepared as an account of work sponsored by an agency of the United States Government. Neither the United States Government nor any agency thereof, nor any of their employees, makes any warranty, express or implied, or assumes any legal liability or responsibility for the accuracy, completeness, or usefulness of any information, apparatus, product, or process disclosed, or represents that its use would not infringe privately owned rights. Reference herein to any specific commercial product, process, or service by trade name, trademark, manufacturer, or otherwise, does not necessarily constitute or imply its endorsement, recommendation, or favoring by the United States Government or any agency thereof. The views and opinions of authors expressed herein do not necessarily state or reflect those of the United States Government or any agency thereof.

\author{
PACIFIC NORTHWEST LABORATORY \\ operated by \\ BATTELLE \\ for the \\ UNITED STATES DEPARTMENT OF ENERGY \\ under Contract DE-AC06-76RLO 1830
}

\begin{tabular}{|c|c|}
\hline \multirow{2}{*}{\multicolumn{2}{|c|}{ Printed in the United States of America }} \\
\hline & \\
\hline \multicolumn{2}{|c|}{$\begin{array}{c}\text { Available from } \\
\text { A }\end{array}$} \\
\hline \multicolumn{2}{|c|}{ National Technical Information Service } \\
\hline \multirow{2}{*}{\multicolumn{2}{|c|}{$\begin{array}{c}5285 \text { Port Royal Road } \\
\text { Springfield, Virginia } 22161\end{array}$}} \\
\hline & \\
\hline \multirow{2}{*}{\multicolumn{2}{|c|}{$\begin{array}{l}\text { NTIS Price Codes } \\
\text { Microfiche A01 }\end{array}$}} \\
\hline & \\
\hline \multicolumn{2}{|c|}{ Printed Copy } \\
\hline & Price \\
\hline Pages & Codes \\
\hline $001-025$ & $\mathrm{~A} 02$ \\
\hline $026-050$ & A03 \\
\hline $051-075$ & $\mathrm{~A} 04$ \\
\hline $076-100$ & A05 \\
\hline $101-125$ & A06 \\
\hline $126-150$ & $\mathrm{~A} 07$ \\
\hline $151-175$ & A08 \\
\hline $176-200$ & A09 \\
\hline $201-225$ & A010 \\
\hline $226-250$ & A011 \\
\hline $251-275$ & A012 \\
\hline $276-300$ & A013 \\
\hline
\end{tabular}


PNL-4912

UC-95f

CHARACTERIZATION OF INDUSTRIAL PROCESS WASTE HEAT AND INPUT

HEAT STREAMS
G. L. Wilfert
H. B. Huber
R. E. Dodge
B. A. Garrett-Price
L. L. Fassbender
E. A. Griffin
D. R. Brown
N. L. Moore

May 1984

Prepared for

the U.S. Department of Energy

under Contract DE-AC06-76RLO 1830

Pacific Northwest Laboratory

Richland, Washington 99352 

. 


\section{EXECUTIVE SUMMARY}

Approximately one-third of the energy consumed in the U.S. is consumed by the manufacturing sector of the economy. Although considerable progress has been made toward increasing energy efficiency in manufacturing, many opportunities for further improvements remain. The greatest opportunities include the use of more efficient process equipment and the recovery and use of industrial waste heat.

The U.S. Department of Energy's (DOE) industrial energy conservation strategy emphasizes high-payoff, long-term, high-risk generic research and development programs. In support of this emphasis, the Pacific Northwest Laboratory was contracted to provide management planning information for the DOE's Waste Heat Recovery Branch.

This report identifies the nature and extent of industrial waste heat associated with the manufacturing sector of the U.S. economy. Specifically, this report addresses the following questions:

- What U.S. industries purchase large amounts of energy?

- What industrial processes generate large amounts of waste heat?

- Which unit operations and types of equipment discharge waste heat streams within these industrial processes?

- What is the energy content of these waste heat streams?

- What are the candidate uses for energy recovered from these waste heat streams?

- Does the temperature of the waste heat need to be reduced or elevated to be useful to candidate users?

- When the energy contained in waste heat streams at $T_{1}$ is compared with candidate energy needs at $T_{2}$, how much energy can be matched up?

Industries purchasing energy at market prices have considerable interest in implementing advanced waste heat recovery equipment to reduce purchased energy costs. In 1980, total manufacturing purchases were estimated to be $\$ 48.2$ billion for some 11.9 quadrillion Btu. Review of industry purchases of 
energy shows that one-third of all purchased energy by the manufacturing sector is purchased by 4 of some 450 four-digit SIC industries. The top 10 four-digit industries account for one-half of all energy purchases.

This report reviews industry energy information, and for planning purposes, estimates the energy content in waste heat streams emanating from 108 energy-intensive industrial processes. In total, these 108 industrial processes account for approximately $70 \%$ of the energy purchased by the manufacturing sector. The energy content in gaseous, liquid, and steam waste streams above $75^{\circ} \mathrm{F}$ is estimated to be nearly 7 quadrillion Btu for the industries addressed.

Of the 108 industrial processes evaluated, the 5 industries with the largest energy content in their gaseous, liquid, and steam waste streams are Petroleum Refining, Blast Furnaces and Steel Mills, Integrated Paper Board Mills, Integrated Paper Mills, and Hydraulic Cement.

Generic types of process equipment are identified in this report and the energy content in gaseous, liquid, and steam waste streams emanating from this equipment are evaluated. The equipment with the largest energy content in output streams includes furnaces, distillation equipment, drying equipment, boilers, and thermal-chemical reactor equipment.

The usefulness of any energy recovered from these waste steams depends largely on the immediate energy input needs of other unit operations in the plant. Thus, the emphasis of this report is to identify matchups between the energy content of waste heat streams and candidate uses. In this assessment only temperature difference and energy content were considered in the matchups.

The temperature spectrum from $80-2300+{ }^{\circ} \mathrm{F}$ is divided into 16 temperature ranges, and the energy content in waste heat and candidate input heat streams is estimated using an industrial energy flow data base. The three temperature ranges containing the largest waste heat are: $80-1190^{\circ} \mathrm{F}(2,522$ trillion Btu/ $y r), 400-499^{\circ} \mathrm{F}$ (598 trillion Btu/yr), and $600-799^{\circ} \mathrm{F}$ (571 trillion Btu/yr). The temperature ranges of the three candidate uses having the largest thermal input needs are: $250-299^{\circ} \mathrm{F}\left(1,244\right.$ trillion Btu/yr), 400-499 ${ }^{\circ} \mathrm{F}$ (592 trillion Btu/yr), and $600-799^{\circ} \mathrm{F}$ (137 trillion Btu/yr). 
In this report, the (thermal) energy of the waste heat stream is compared with candidate input heat requirements. Matrices are developed to compare this energy by temperature ranges. The resultant matrix identifies 256 source/ sink (waste heat/candidate input heat) temperature combinations. The maximum amount of energy that can be transferred from a source to a sink is the lesser of the input energy and the ouput energy for that source/sink combination. The maximum energy transferable and the temperature differential between the source and sink is useful when considering the applicability of specific waste heat recovery technologies.

Tables summarizing the maximum energy transferable from source to sink are provided for three classes of heat streams:

- liquid and gaseous heat streams combined

- liquid heat streams only

- gaseous heat streams only.

For each of these classes, the source/sink temperature combination with the largest energy matchup is evaluated to identify contributing industrial processes and generic types of equipment. 


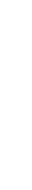




\section{CONTENTS}

EXECUTIVE SUMMARY

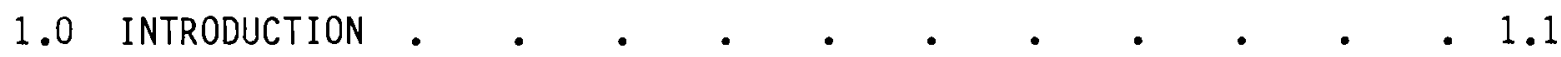

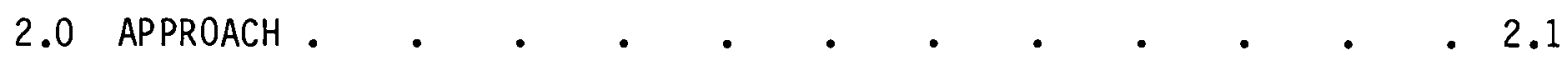

CLASSIFICATION OF INDUSTRIAL ACTIVITY $\quad \cdot \quad \cdot \quad \cdot \quad \cdot \quad \cdot 2.1$

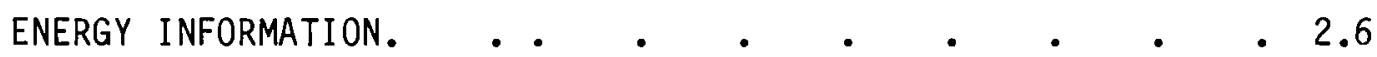

TYPICAL PLANT OPERATIONS • • • • • • • • • • $\quad$ • 2.6

COMPILATION OF ENERGY DATA BY TEMPERATURE RANGE $• \quad$ • $\quad 2.7$

ENERGY SOURCE/SINK INFORMATION $• . \quad \cdot \quad \cdot \quad \cdot \quad \cdot \quad \cdot 2.8$

3.0 INDUSTRY ENERGY INFORMATION

SPECIFIC INDUSTRY ENERGY INFORMATION $\quad \cdot \quad \cdot \quad \cdot \quad \cdot \quad \cdot 3.2$

Sector-Wide Information . • • • • • • 3.2

Individual Industry Information . $\quad . \quad$. $\quad . \quad 3.4$

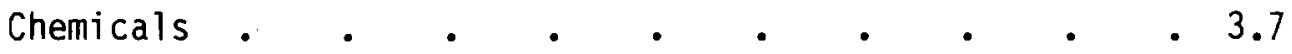

Industrial Organics . $\quad . \quad$. . . . . 3.10

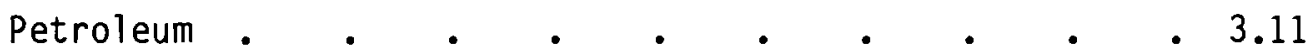

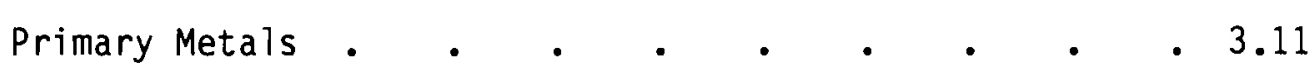

Paper and Allied Products . . . . . . 3.12

Stone, Clay and Glass . . . . . . . . 3.12

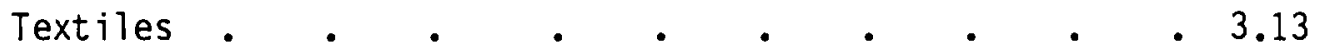

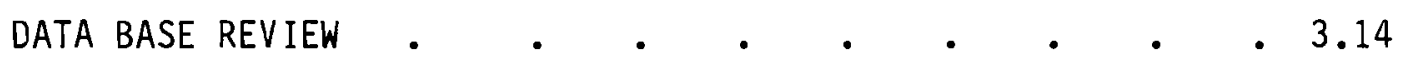

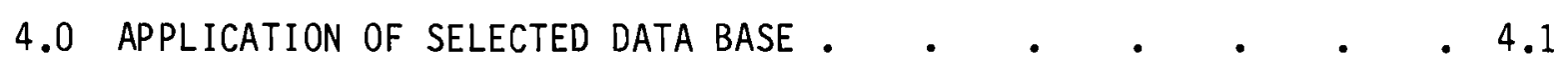

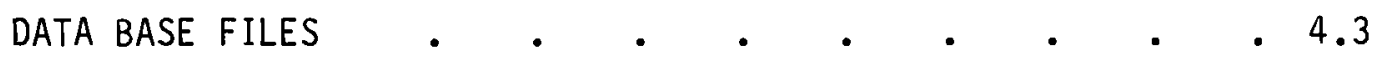

Updating Drexel Industrial Data Files . • • . 4.4

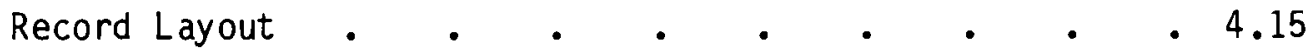

GENERAL CAPABILITIES OF SYSTEM 2000 DBMS $\quad$ • • • $\quad$ • 4.21

DATA BASE EXPANSION/ADJUSTMENTS . . • • • • . 4.23

Industry Production Information . . . . . 4.24

Adjustment Factors • • • . • • • . 4.32

WASTE HEAT STREAM ENERGY CONTENT • • • • • • • • 4.33

Waste Heat by Industry . • . • • • . 4.34

Waste Heat by Type of Equipment . . . . . 4.35 
5.0 SUBSTITUTING WASTE HEAT ENERGY FOR PROCESS HEAT NEEDS • • • 5.1

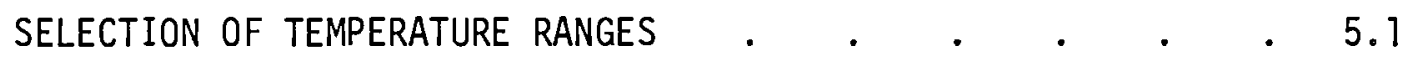

ENERGY STREAM DATA.$\quad \cdot \quad \cdot \quad \cdot \quad \cdot \quad \cdot \quad \cdot \quad \cdot \quad \cdot 5.3$

Compiled by Type of Waste Heat . . . . . . . 5.3

Compiled by Type of Equipment . . . . . . 5.9

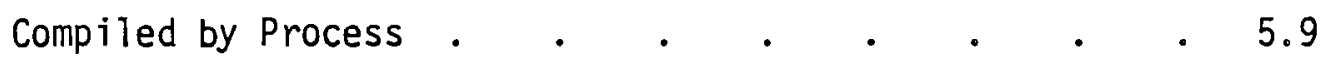

MATCHUP OF WASTE AND INPUT HEAT STREAMS . $\quad \cdot \quad \cdot \quad \cdot 5.9$

6.0 WASTE HEAT RECOVERY OPPORTUNITIES . . . . . . . . . . 6.1

SUMMARY OF SOURCE/SINK MATCHUPS . . . . . .

HEAT BOOSTING/HEAT EXCHANGER OPPORTUNITIES • • • $\cdot 6.7$

7.0 CHARACTERIZATION OF HIGH-ENERGY SOURCE/SINK COMBINATIONS • $~ 7.1$

COMBINED LIQUID AND GASEOUS HEAT STREAMS. • • • .7 .3

GASEOUS WASTE HEAT STREAMS $\cdot$.

LIQUID WASTE HEAT STREAMS. $. \quad . \quad . \quad . \quad . \quad \cdot \quad \cdot 7.8$

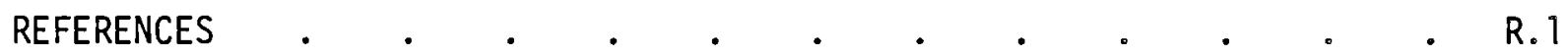

APPENDIX A - CHEMICALS - SIC 28 . . . . . . . . A. 1

APPENDIX B - PETROLEUM REFINING AND RELATED INDUSTRIES - SIC 29 • B. 1

APPENDIX C - PRIMARY METAL INDUSTRIES - SIC 33 . . . . . . . C.

APPENDIX D - PAPER AND ALLIED PRODUCTS - SIC 26 . • . . . . D. 1

APPENDIX E - STONE, CLAY AND GLASS - SIC 32 . . . . . . . E.1

APPENDIX F - TEXTILES - SIC 22 . $2 . \quad . \quad . \quad . \quad . \quad . \quad$. $\quad$ F.1 


\section{$\underline{\text { TABLES }}$}

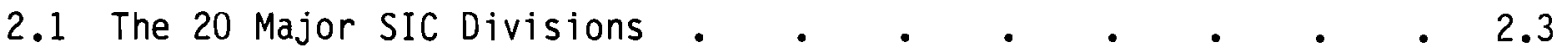

3.1 Percent of Total Energy Consumed by A11 Manufacturing

Industries for Selected Fuels and Purchased Electric Energy . 3.1

3.2 U.S. Manufacturing 1974 Energy Consumption by Primary Fuel and Functional End Use. . . . . . . . . . . 3.3

3.3 Energy Purchases by Two-Digit Level Industries . $\quad$ • $\quad$ • $\quad 3.4$

3.4 Energy Purchases by the Top 80 Four-Digit Level Industries $\quad 3.5$

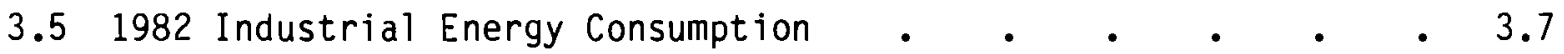

3.6 Waste Heat Potential in the Chemical Industry . $\quad . \quad$ e $\quad 3.8$

3.7 Waste Heat Stream Characteristics of Industrial

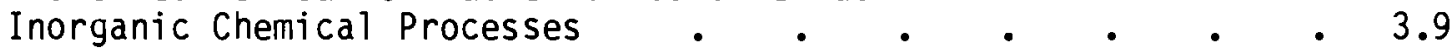

3.8 Waste Heat Streams from Ammonia Production . . . . . 3.10

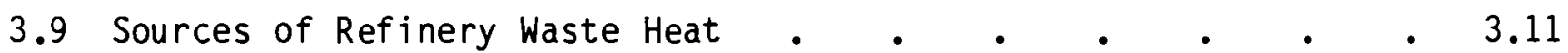

3.10 Annual Waste Heat Discharged by Primary Metals Industries . $\quad 3.11$

3.11 Waste Heat Streams - Pulp Mills . • • • • • • . 3.12

3.12 Sources and Temperatures of Waste Heat

from Stone, Clay and Glass Industries . . . . . . . 3.13

3.13 Quantity of Waste Heat Discharged from Cement, Lime
and Glass Production . . . . . . . . . . 3.14

3.14 Sources of Waste Heat from Textile Mill Operations . $\quad$ • 3.14

3.15 Twenty-Five Industrial Energy Data Bases and Summary of

3.16 Eleven Industrial Energy Data Bases and Summary

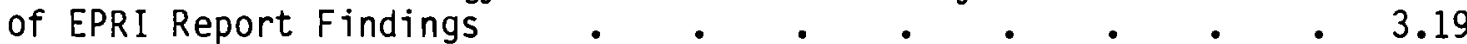

Process Industries With Significant Changes Between 1979
Tape and 1980 Drexel Manual ...
.

4.2 Changes Made to Update the 1979 Drexel Data Tape $\quad$ • $\quad 4.5$

4.3 Definition of Drexel Data Elements . . . . . . . . 4.17

4.4 Drexel Data Element Codes . . • • • • • • . . 4.18

4.5 Schema for Updated System 2000 Drexel Data Base . • . 4.20

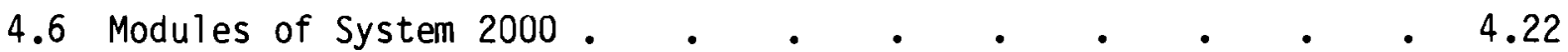

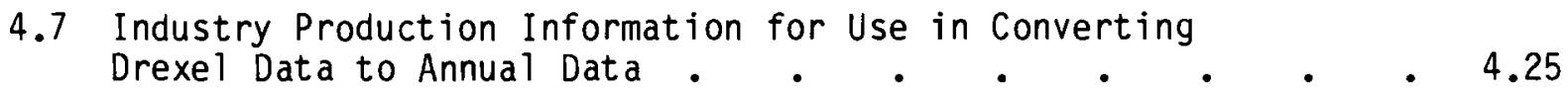


4.8 Comparison of Industrial Production Estimates . $\quad . \quad$. 4.29

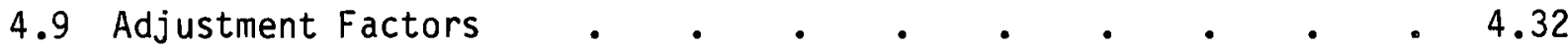

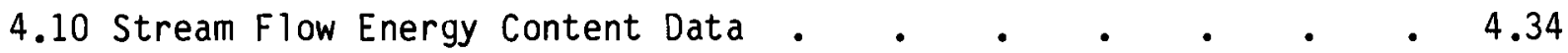

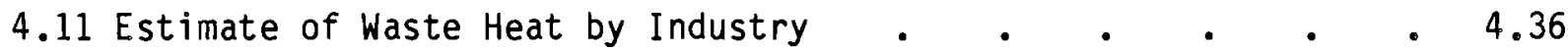

4.12 Estimate of Waste Heat by Type of Process Equipment . . . 4.39

5.1 Waste Heat/Input Energy Temperature Ranges . . • . . 5.2

5.2 Energy Content of Heat Streams by Temperature Range . • • 5.3

5.3 Waste Heat Energy Available $-10^{12}$ Btu/yr. • . • 5.4

5.4 Energy Content of Waste Heat and Input Heat Streams

5.5 Energy Content of Waste Heat and Input Heat Streams
for Industrial Process 2611-1, Pulp Mills . . . . . 5.7

5.6 Liquid and Gaseous Waste Heat Stream Utility Matrix -
Process 2011-1, Meat Packing . . . . . . . 5.8

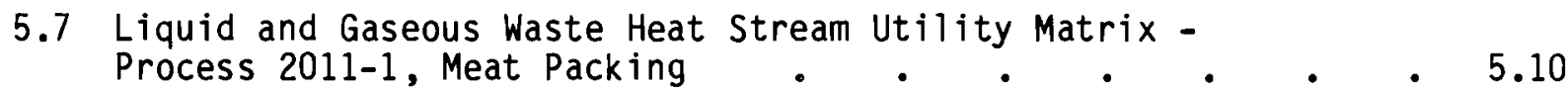

5.8 Liquid and Gaseous Waste Heat Stream Utility Matrix -

5.9 Liquid and Gaseous Waste Heat Stream Utility Matrix -
Process $2011-1$, Meat Packing . 5.13

5.10 Liquid and Gaseous Waste Heat Stream Utility Matrix -

Process 2611-1, Pulp Mills . . . . . . . . 5.14

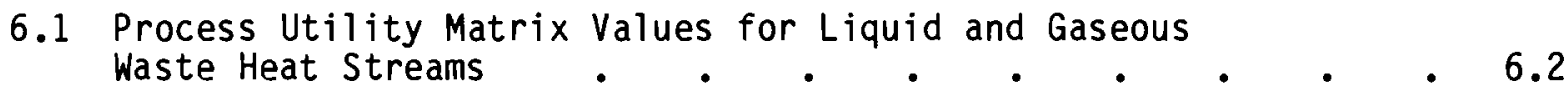

6.2 Summary of 108 Source/Sink Matchups: Combined Liquid
and Gaseous Heat Streams . $\quad . \quad . \quad . \quad . \quad . \quad .6 .4$

6.3 Summary of 108 Source/Sink Matchups: All Gas Heat Streams $\quad 6.5$

6.4 Summary of 108 Source/Sink Matchups: All Liquid Heat Streams $\quad 6.6$

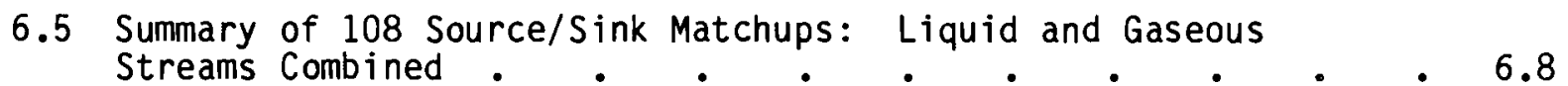

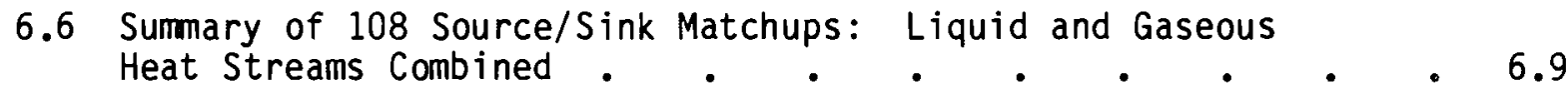

6.7 Summary of 108 Source/Sink Matchups: Liquid and Gaseous
Heat Streams Combined

7.1 Utility Summary Cells with the Highest Energy Values . • • 7.2 
7.2 Industry Processes Contributing to the $\left(80-119^{\circ} \mathrm{F}\right.$ Output

Stream) $/\left(400-499^{\circ} \mathrm{F}\right.$ Input Stream) Cell in the Combined

Liquid and Gaseous Heat Stream Utility Matrix Summary • • 7.3

7.3 Major Unit Operations Supplying the Energy Sources and Sinks

for the $\left(80-119^{\circ} \mathrm{F}\right.$ Output Stream)/(400-499 $\mathrm{F}$ Input Stream)

Cell in the Combined Liquid and Gaseous Heat Stream

Utility Matrix . • • • • • • • • • 7.4

7.4 Industry Processes Contributing to the $\left(80-119^{\circ} \mathrm{F}\right.$ Output

Stream $) /\left(250-299^{\circ} \mathrm{F}\right.$ Input Stream) Cell in the Combined

Liquid and Gaseous Heat Stream Utility Matrix Summary

7.5 Major Unit Operations Supplying the Energy Sources and Sinks

for the $\left(80-119^{\circ} \mathrm{F}\right.$ Output Stream) $\left(250-299^{\circ} \mathrm{F}\right.$ Input Stream)

Cell in the Combined Liquid and Gaseous Heat Stream

Utility Matrix Summary.

7.6 Industry Processes Contributing to the $\left(400-499^{\circ} \mathrm{F}\right.$ Output

Stream)/(250-299 F Input Stream) Cell in the Combined

Liquid and Gaseous Heat Stream Utility Matrix Summary

7.7 Major Unit Operations Supplying the Energy Sources and Sinks

for the $\left(400-499^{\circ} \mathrm{F}\right.$ Output Stream $) /\left(250-299^{\circ} \mathrm{F}\right.$ Input Stream)

Cell in the Combined Liquid and Gaseous Heat Stream

7.8 Industry Processes Contributing to the $\left(212-249^{\circ} \mathrm{F}\right.$ Output

Stream)/(250-299 $\mathrm{F}$ Input Stream) Cell in the Combined

Liquid and Gaseous Heat Stream Utility Matrix Sumary

7.9 Major Unit Operations Supplying the Energy Sources and Sinks

for the $\left(212-249^{\circ} \mathrm{F}\right.$ Output Stream)/(250-299 $\mathrm{F}$ Input Stream)

Cell in the Gas Waste Heat Stream Utility Matrix Summary

7.10 Industry Processes Contributing to the $\left(400-499^{\circ} \mathrm{F}\right.$ Output

Stream)/(250-299 $\mathrm{F}$ Input Stream) Cell in the Combined

Liquid and Gaseous Heat Stream Utility Matrix Summary

Major Unit Operations Supplying the Energy Sources and Sinks for the $\left(400-499^{\circ} \mathrm{F}\right.$ Output Stream)/(250-299 $\mathrm{F}$ Input Stream)

Cell in the Gaseous Waste Heat Stream Utility Matrix Summary

7.12 Industry Processes Contributing to the $\left(80-119^{\circ} \mathrm{F}\right.$ Output Stream $) /(140-211 \mathrm{~F}$ Input Stream) Cell in the Liquid Heat Stream Utility Matrix Summary

7.13 Major Unit Operations Supplying the Energy Sources and Sinks for the $\left(80-119^{\circ} \mathrm{F}\right.$ Output Stream $) /\left(140-211^{\mathrm{F}}\right.$ Input Stream)

Cell in the Liquid Waste Heat Stream Utility Matrix Summary

7.14 Industry Processes Contributing to the $\left(140-211^{\circ} \mathrm{F}\right.$ Output Stream)/(140-211 F Input Stream) Cell in the Liquid Heat Stream Utility Matrix Summary

Major Unit Operations Supplying the Energy Sources and Sinks

for the $\left(140-211^{\mathrm{F}}\right.$ Output Stream) $\left(140-211^{\mathrm{F}}\right.$ Input Stream)

Cell in the Liquid Waste Heat Stream Utility Matrix Sumary 


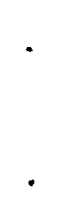




\section{FIGURES}

2.1 Process Flow - Pulp Mills, SIC 2611-1 • • • • • • • 2.5

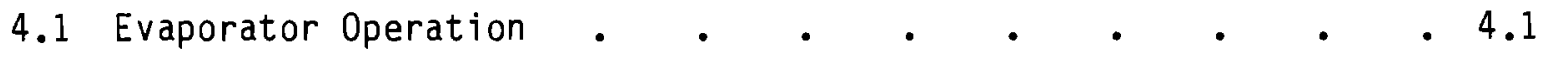

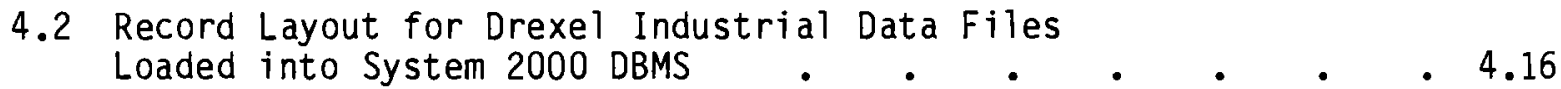

6.1 Development of the Source/Sink Summation Table $\quad$. $\quad$ • $\quad 6.3$ 


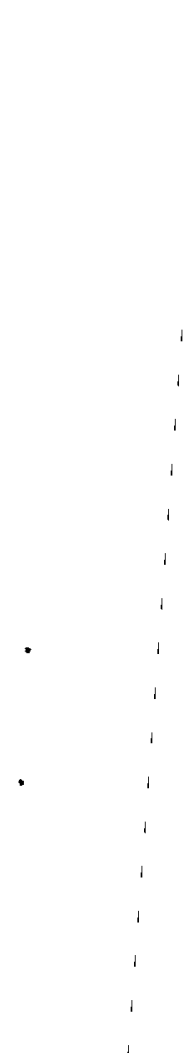


CHARACTERIZATION OF INDUSTRIAL PROCESS WASTE HEAT AND INPUT HEAT STREAMS

\subsection{INTRODUCTION}

\section{OBJECTIVES}

The research discussed herein addresses five objectives: 1) review the 1979 Drexel industrial data base and specify its strengths and weaknesses; 2) update the data base with 1980 data; 3) extend the data base with industry production information; 4) estimate the energy content of waste heat streams within a set of temperature ranges; and 5) identify for specific industries the unit operations that make the most important energy contribution to the liquid and gaseous waste stream totals. All of these objectives were met and are discussed fully in this report. The report also identifies candidate energy uses (sinks) that may use energy received from waste heat streams and matches these thermal energy needs to the thermal energy present in waste heat streams.

\section{BACKGROUND}

In 1974, the manufacturing sector purchased an estimated 13.5 quadrilion Btu (13.5 quads). Since the $0 i 1$ embargo of 1973-74 and its related energy shortages and higher prices, the manufacturing sector has aggressively pursued energy conservation. In 1980, total manufacturing sector purchases dropped to an estimated 11.9 quads, about 1.6 quads below the 1974 level (Department of Commerce 1982).

The immediate reaction to the energy shortage was to curtail unnecessary energy use, for example, curtailing nonessential energy uses. However, industry has since moved toward improving the management of energy systems by using the available energy more efficiently rather than "doing without." The results have been significant, and many companies have found that through conscientious management, energy requirements can be reduced by 10 to $30 \%$ without major capital investments or reduced production. Although most industries have tried some form of energy management, many opportunities for further improvements 
remain. In the long term, the greatest opportunities for improving energy efficiency are to substitute more energy-efficient equipment in industrial processes and to more fully use process waste heat, the subject of this report. Waste heat herein is defined to be the thermal energy from streams of hot air, hot exhaust gases, and hot liquids that leave industrial processes and enter the environment at temperatures high enough above the ambient temperature to permit the recovery of some fraction of that energy for useful purposes.

The Department of Energy's (DOE) revised R\&D strategy emphasizes highpayoff, long-term, high-risk generic R\&D programs. In support of this emphasis, the Pacific Northwest Laboratory (PNL) was contracted to provide management planning information for the Waste Heat Recovery Branch. This new planning effort revealed that in general the existing literature did not have the readily available data required to identify additional long-term R\&D opportunities for industrial waste heat recovery. This information is necessary, however, to identify future technology development programs that will significantly impact a wide spectrum of industries and to insure that federal research does not duplicate privately sponsored research.

\section{REPORT ORGANIZATION}

This project, Waste Heat Recovery Analysis, consists of two tasks. The purpose of Task 1 is to identify and evaluate waste heat streams from industrial processes, to estimate, for planning purposes, the energy content in waste streams and to match this energy with candidate uses. To obtain these estimates, many data bases were reviewed and the Drexel data base was selected. The purpose of Task 2 is to provide information on the availability of commercial waste heat recovery equipment and to identify recent trends in the equipment design and operation.

This Task 1 report provides an overview of the energy content of waste heat streams for specific industrial processes. By analyzing the energy content of waste heat streams (source) and the thermal energy input requirements for specific processes (sink), we estimate the relative magnitude of the energy that could be used through waste heat recovery technology. Although 
this assessment contains several assumptions and caveats, the energy source/ energy sink information provides new insights into the opportunities for waste heat recovery equipment.

This report is organized into seven chapters and six appendices. Chapter 2.0 describes the approach used in the assessment and discusses several limitations and caveats to be considered when using the data to estimate waste heat recovery opportunities. Review of energy use and industrial energy flow data bases is discussed in Chapter 3.0. Chapter 4.0 discusses the selection of the Drexel data base and its subsequent update, expansion and adjustment for use in this assessment. Chapter 5.0 identifies the temperature ranges used in compiling energy content data; compiles energy content data by type of waste stream, type of equipment and by industrial process; and describes the process for matching up the energy content of waste heat streams with input heat streams. Chapter 6.0 summarizes the source/sink information across 108 industry processes (these processes are estimated to represent approximately $70 \%$ of the energy purchased by industry). Chapter 7.0 looks at specific high-energy-content sources/sink situations and identifies the key industries and generic types of equipment involved.

The appendices are separated into discussions of six industries: Chemicals; Petroleum Refining and Related Industries; Primary Meta 7 Industries; Paper and Allied Products; Stone, Clay and Glass; and Textiles. Each appendix provides a process description, a discussion of major heat transfer operations, and a characterization of waste heat streams within that industry. 


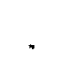

. 


\subsection{APPROACH}

Approximately one-third of the energy used in the U.S. is consumed by manufacturing processes. To evaluate how energy is used in industry and to estimate the extent to which advanced waste heat recovery technology may be applicable is complex and difficult. The manufacturing sector covers a broad spectrum of industries and products encompassing hundreds of thousands of establishments, each having its own set of process and management characteristics.

In this chapter, the four levels of the Standard Industrial Classification (SIC) system used to classify the thousands of establishments are explained as they relate to the manufacturing sector. Also, the approach used in assessing the energy content of waste heat streams is discussed. Finally, our assumptions and the limitations that should be considered when using the data to identify potential research opportunities for waste heat recovery are discussed.

\section{CLASSIFICATION OF INDUSTRIAL ACTIVITY}

Various classification systems have been developed to organize information on the broad spectrum of manufacturing activities, including systems for classifying according to products, occupations, and enterprises. The Standard Industrial Classification (SIC) of Establishments, however, is especially useful when addressing industrial processes because it classifies manufacturing establishments by their types of activities.

Three principals are used to classify establishments having similar activities (Department of Commerce 1972).

1. The classification structure should conform to the existing structure of American industry.

2. Establishments should be classified according to their primary activity.

3. The group of establishments constituting a classification category must be statistically significant in the number of persons employed, the volume of business done, and other measures of economic activity.

Because the SIC system is broadly based and well defined, it is widely used in tabulating industry information. Its organizational structure 
facilitates the collection, tabulation, presentation and analysis of industrial activity information. As such, the SIC code is used by various U.S. government agencies, state agencies, trade associations and private research organizations. For example, the U.S. Department of Commerce compiles manufacturing sector information at the two-, three- and four-digit level for its Census of Manufactures and for its Annual Survey of Manufactures publications.

In the SIC system, each manufacturing establishment is assigned an industry code (usually at the four-digit level) on the basis of its primary activity. The primary activity is determined by its principal product or group of products. The coding system has been set up so that all establishments primarily engaged in the same kind of economic activity are classified in the same four-digit industry, regardless of their type of ownership. The SIC coding scheme is hierarchical, consisting of two-, three-, and four-digit codes providing increasingly detailed industrial classification.

The manufacturing sector is identified by 20 major industrial groups defined by the two-digit SIC numbers 20 through 39 (Table 2.1). Each of these major groups of industries contains 5 or more three-digit industries. For example, Food and Kindred Products (Major Group 20) is subdivided into 9 threedigit industries:

201 Meat Products

202 Dairy Products

203 Canned and Preserved Fruits and Vegetables

204 Grain Mill Products

205 Bakery Products

206 Sugar and Confectionary Products

207 Fats and 0 ils

208 Beverages

209 Miscellaneous Food Preparation and Kindred Products. The digit "9" in the third or fourth position of the SIC code designates residual establishments whose activities are not homogeneous with the other specified activities identified within the three- or four-digit group of industries. 


\section{TABLE 2.1 The 20 Major SIC Divisions}

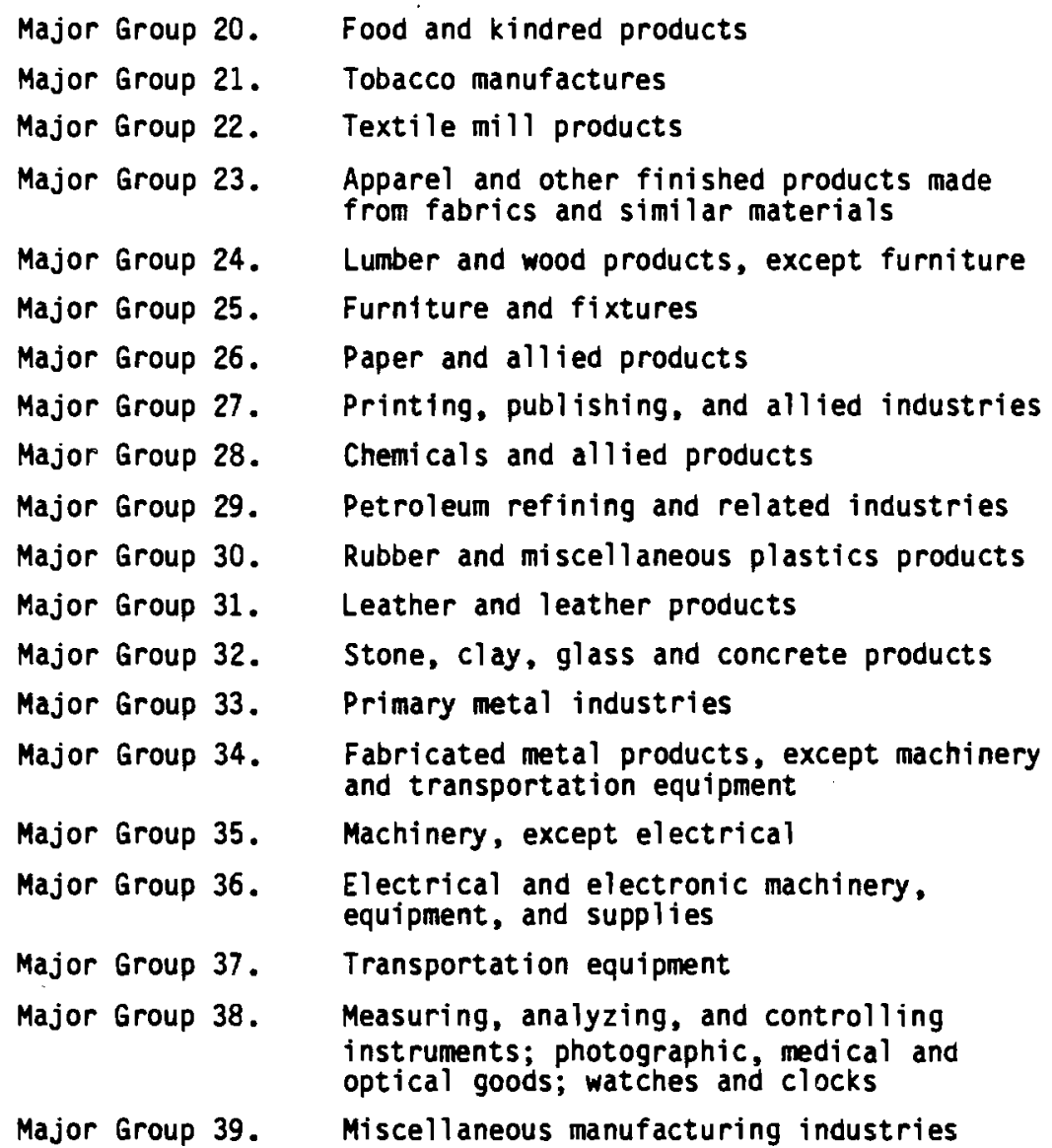

The four-digit level of classification is fairly narrow and specifically defined. An example of four-digit activities are those that comprise SIC 201Meat Products:

2011 Meat packing

2013 Sausage and other prepared meat products

2016 Poultry dressing plants

2017 Poultry and egg processing.

In this example, meat packing plants (SIC 2011) are defined as establishments primarily engaged in the slaughtering, for their own account or on a contract basis for the trade, of cattle, hogs, sheep, lambs, and calves for meat to be 
sold or to be used on the same premises in canning and curing, and in making sausage, lard and other products (Department of Commerce 1972). Establishments primarily engaged in killing, dressing, and packing poultry, rabbits, and other small game animals would not be included in SIC 2011, but are classified as Industry 2016. Also, the establishments primarily engaged in killing and processing horses and other nonfood animals would not be included in 2011, but are classified as SIC 2047, which is outside of the 201 series. Establishments primarily engaged in manufacturing sausages and meat specialties from purchased meats are classified in Industry 2013, and establishments primarily engaged in canning meat for baby food are classified 2032, which is from another threedigit SIC category, Canning and Preserving Fruits and Vegetables.

The four-digit level of the SIC code provides the lowest level of industry detail covered by the censuses and surveys of manufacturers. However, below this level of industry detail we can identify individual processes used to produce specific products and, within a particular process, the unit operations that make up a process. For example, there are three active processes for the production of pulp, SIC 2611 - Pulp mills (manufacture of pulp from wood or from other materials such as rags, linters, waste paper, and straws). In the data base that was selected for this assessment, different processes are identified by suffixing a fifth digit to the four-digit SIC number, for example as follows:

2611-1 Pulp mills - Kraft process

2611-2 Pulp mills - sulfite process

2611-3 Pulp mills - mechanical process.

Information at the process level is required to assess the usefulness of energy in waste heat streams. However, information even below the process level is also important. At this level, the specific unit operation level, energy streams take on specific characteristics that must be evaluated to identify opportunities for waste heat recovery. Figure 2.1 shows seventeen unit operations that are identified with the Kraft process method of producing pulp (Drexel University 1980). 


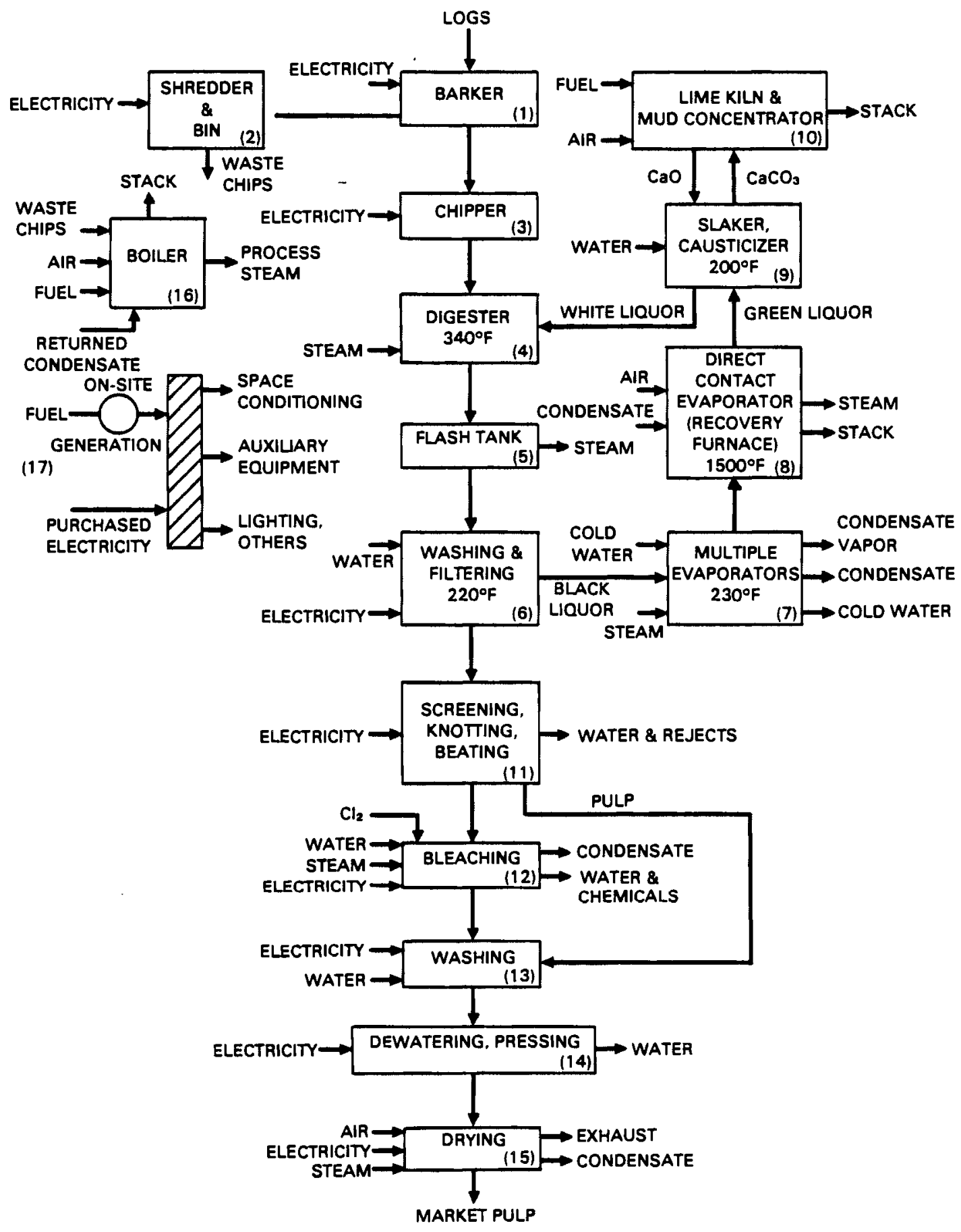

FIGURE 2.1 Process Flow - Pulp Mills, SIC 2611-1 (DOE 1980) 


\section{ENERGY INFORMATION}

As discussed above, an industrial process can be defined as a set of unit operations combined to process raw material into finished products. Each unit operation performs a specific function in the process. The material and energy stream flows coming into and out of each unit operation describe the nature of that process step. Outlet flow streams that contain heat above $80^{\circ} \mathrm{F}$ and that are released to the environment are classified as waste heat energy streams. Examples of waste heat streams are boiler stack gases (unit operation 16 in Figure 2.1), dryer exhaust (unit operation 15), dewatering water (unit operation 14), evaporator steam (unit operation 8), etc. In this study, the procedure used to evaluate waste streams at the unit operation level is to quantify the energy content of these energy streams for a typical plant.

For this planning assessment, we need to estimate for a full year the energy content of waste heat streams (sources) and input heat streams (sinks). Therefore, typical plant energy flow information (in Btus per unit operation) must be combined with industry production information at the national level to obtain annual energy flow information. We used industry production information provided by the Census of Manufactures and the DOE (discussed in Chapter 4.0). To estimate annual energy content of waste heat streams, energy flow information for typical plants at the four-digit SIC level was compiled in a data base. This PNL-modified data base provides the annual energy content for individual industry energy streams and forms the basis of our analysis on the energy content in waste and input heat streams.

\section{TYPCIAL PLANT OPERATIONS}

Unfortunately, the diversity of industrial operations even at the fourdigit SIC level makes an analysis of intra-industry energy flow extremely complex and difficult. Not only are there many plants to be considered, but most manufacturing plants have unique characteristics that influence energy flow patterns. The problem is compounded by the lack of reliable data and the difficulties in getting access to individual plants to develop primary data. Thus, 
even at the four-digit level, the lowest level of industry information, plants vary significantly in size, structure, operating procedures and energy flow.

A common approach to conducting broad based industry evaluations has been to develop a model by describing the operation of a typical plant for the industry. Our literature review has not produced an alternative to using a "typical plant" in broadly assessing the operation of an industry or particular process. Typical plant information generally substitutes for specific plant data because collecting data for every major industrial plant is restricted by cost and legal access constraints.

Thus, typical plant data provide the best information available for analyzing, generically, the energy flow patterns of industry, although the findings of such an analysis may be only partially valid for any specific plant. The typical plant approach is applicable when assessing for program planning purpose the energy content for waste heat and input heat streams within general temperature ranges. This temperature range information can be used to identify potential matchups between waste heat and input heat needs. The matchup (utilization) information can then be used to identify unit operations where opportunities may exist for using advanced waste heat recovery technology. Based on this information, specific plant engineering studies can be used to gather engineering data for waste heat recovery equipment design and operation information. The data in this assessment describing a typical plant's operation is, of course, not adequate nor intended to be used for detailed engineering design of heat recovery equipment. Engineering design of information can only be obtained through a site-specific engineering assessment.

\section{COMPILATION OF ENERGY DATA BY TEMPERATURE RANGE}

The usefulness of recovered energy from waste heat streams depends largely on the need of that energy in nearby unit operations. Recovered energy from waste heat may displace part of or all of the energy input needs for a specific unit operation within an industrial plant. The temperature differential between waste heat streams and input heat streams that must be overcome to make the energy in waste heat streams useful is one factor addressed in this assessment. 
The information about the temperature differential between sources of energy and sinks for reclaimed energy is useful when considering the applicability of specific waste heat recovery technologies. Thus, our research approach involved identifying temperature differences between waste heat and input heat streams and assessing the maximum amount of energy that might be transferred. These two parameters, temperature difference and energy transfer, are key factors in evaluating specific heat recovery opportunities.

Sixteen temperature ranges were selected for analysis and the waste heat data base was queried to obtain the energy content of waste heat streams and input energy streams within each temperature range.

\section{ENERGY SOURCE/SINK INFORMATION}

The energy content of waste heat streams and energy input needs within each of the 16 temperature ranges is the basis for our evaluating the uses of recovered waste heat. To identify situations where energy from waste heat streams may be substituted for input heat energy, 108 industrial processes were examined. For each process separate matrices were developed that match up waste heat energy sources with input heat sinks across the 16 temperature ranges. Matrices were developed for three classes of heat streams:

- liquid and gaseous input and output heat streams combined

- liquid input and output heat streams only

- gaseous input and output heat streams only.

As Chapter 6.0 will discuss, the use of waste heat energy identified for each specific process is summed across all industries. This summary represents the maximum amount of waste heat that can be used in each of 256 matrix cells defined by a source and sink temperature. Chapter 6.0 concentrates on those cells in the summary tables having the highest energy content. Because these cells have high energy content, it is valuable to know which industries, and which unit operations within those industries contribute importantly to the cells energy content value. Chapter 6.0 ends with the provision of this information. 


\subsection{INDUSTRY ENERGY INFORMATION}

In this chapter, energy use by the manufacturing sector is reviewed in terms of purchased fuels. Also, operation and energy-use characteristics of 6 two-digit SIC industries are summarized based on an extensive literature review. A more detailed write up for each of these 6 industries is provided in Appendices A through F. Finally, various industry-oriented energy data bases are briefly reviewed.

The 1980 Annual Survey of Manufactures (Department of Commerce 1982) indicates that 11.9 quads of purchased fuels were consumed by the manufacturing sector in 1980. These purchases were from a variety of primary and secondary energy sources including natural gas, crude petroleum products, coal, and hydro and nuclear power. Table 3.1 shows the relative importance of each energy source. Natural gas is by far the largest source of energy for the manufacturing sector. It contributed $51 \%$ of energy purchased in 1980 , whereas electricity provided $19 \%$ of the energy purchased. Comparing the right hand column of the table to the 1980 value shows how the mix of fuel has changed since 1974 .

TABLE 3.1 Percent of Total Energy Consumed by All Manufacturing Industries for Selected Fuels and Purchased Electric Energy (U.S. Department of Commerce 1982)

\begin{tabular}{|c|c|c|}
\hline Type of Fuel & $\begin{array}{l}\text { Percen } \\
\text { Energy } \\
1980\end{array}$ & $\begin{array}{c}\text { Total } \\
\text { chased } \\
1974 \\
\end{array}$ \\
\hline Residual fuel oil & 8 & 9 \\
\hline Bituminous coal, lignite, anthracite & 12 & 10 \\
\hline Coke and breeze & 4 & 3 \\
\hline Disttllate fuel oil & 2 & 5 \\
\hline Natural gas & 51 & 54 \\
\hline Purchased electric energy & 19 & 15 \\
\hline $\begin{array}{l}\text { Other fuels (includes liquefied petro- } \\
\text { leum gases) }\end{array}$ & 4 & 3 \\
\hline
\end{tabular}


Total energy purchased by the manufacturing sector in 1974 was 13.5 quads or 1.6 quads above the 1980 purchases. Over this six-year period, purchased fuels have favored electricity and coal, whereas the relative energy purchases of natural gas and fuel oil have declined.

An analysis of the functional end uses of the primary fuels consumed by the manufacturing sector in 1974 indicates that industrial process heat is by far the most important end use in the manufacturing sector (SRC 1980). Of the total energy consumed in 1974, 26.3\% was used for direct heat, $21.0 \%$ was used for process steam, and $19.2 \%$ for feed stocks (Table 3.2). Although industry consumption of purchased fuels has changed somewhat since 1974, direct heat, process steam and fuel feed stocks end uses still account for the majority of the fuels consumed by the manufacturing sector.

SPECIFIC INDUSTRY ENERGY INFORMATION

As discussed in Chapter 2.0, the manufacturing sector of the U.S. economy includes a broad spectrum of industries and products. The SIC code subdivides the manufacturing sector into 20 major groups identified by two-digit SIC numbers, 20 through 39. (SIC numbers and titles for these major groups are shown in Table 3.3.) Each of the major groups of industries is subdivided into the three-digit SIC divisions, and each of these divisions is subdivided further, identifying the four-digit SIC industries. The four-digit level provides the finest industry detail. A total of 452 four-digit SIC industries are identified within the SIC coding system.

Sector-Wide Information

Table 3.3 shows energy purchases by two-digit level industries. Of the 11.9 quads of energy purchased in 1980 by the manufacturing sector, Chemicals (SIC 28), Primary Metals (SIC 33), and Paper and Allied Products (SIC 26) accounted for $52.8 \%$. As the table shows, the top 10 two-digit industries accounted for $91.4 \%$ of all energy purchased by the manufacturing sector.

One-third of all purchased energy is purchased by 4 of the 452 four-digit industries. The top 10 four-digit industries account for one-half of all energy purchased by the manufacturing sector. Table 3.4 shows the purchased 
TABLE 3.2 U.S. Manufacturing 1974 Energy Consumption by Primary Fuel and Functional End Use (SERI/SRC 1980)

\begin{tabular}{|c|c|c|c|c|c|c|c|c|c|c|c|c|}
\hline Functional End Use & Coal & Coke & $\begin{array}{l}\text { Dist. } \\
\text { Fuel } \\
011 \\
\end{array}$ & $\begin{array}{l}\text { Res. } \\
\text { Fuei } \\
011\end{array}$ & $\begin{array}{l}\text { Misc. } \\
\text { Petro. } \\
\text { Products }\end{array}$ & LPG & $\begin{array}{c}\text { Natural } \\
\text { Gas } \\
\end{array}$ & $\begin{array}{l}\text { 0ther } \\
\text { Gas }\end{array}$ & Hydro & Elec. & $\begin{array}{l}\text { Other } \\
\text { Energy }\end{array}$ & Total \\
\hline Space heating & 0.1 & & 1.1 & 0.5 & & & 0.6 & & & 0.1 & & 0.3 \\
\hline Space cooling & & & & & & & & & & 1.3 & & 0.2 \\
\hline Space condtitioning & & & & & & & & & & 0.1 & & NS \\
\hline \multicolumn{13}{|l|}{ NSK/NEC } \\
\hline Lighting & & & & & & & & & & 1.8 & & 0.2 \\
\hline $\begin{array}{l}\text { Total direct heat } \\
\text { be } 1 \mathrm{O}^{\circ} 600^{\circ} \mathrm{F} \\
600^{\circ} \text { to } 1000^{\circ} \mathrm{F} \\
1000^{\circ} \mathrm{F} \text { to } 1500^{\circ} \mathrm{F} \\
\text { above } 1500^{\circ} \mathrm{F}\end{array}$ & 6.5 & 1.8 & $\begin{array}{r}26.2 \\
3.4 \\
5.2 \\
1.4 \\
2.3\end{array}$ & $\begin{array}{r}38.7 \\
3.8 \\
10.6 \\
0.7 \\
1.1\end{array}$ & $\begin{array}{l}32.0 \\
31.2\end{array}$ & $\begin{array}{l}2.6 \\
0.5 \\
1.6 \\
0.1\end{array}$ & $\begin{array}{r}38.1 \\
3.6 \\
9.0 \\
3.3 \\
1.8\end{array}$ & $\begin{array}{r}80.7 \\
12.4 \\
43.7 \\
2.9\end{array}$ & 1.7 & $\begin{array}{l}1.5 \\
0.1\end{array}$ & 3.1 & $\begin{array}{r}26.3 \\
2.5 \\
7.0 \\
3.6 \\
0.8\end{array}$ \\
\hline Direct heat, NSK & & 1.8 & 13.9 & 18.0 & 0.9 & 0.2 & 19.3 & 21.7 & 1.7 & 2.7 & 1.8 & 11.8 \\
\hline Raw Material & & 88.7 & & & 67.8 & 91.0 & 6.7 & & & & 2.8 & 19.3 \\
\hline Process steam & 15.0 & 0.1 & 25.6 & 37.1 & & 0.8 & 23.9 & 21.5 & & & 50.5 & 21.0 \\
\hline Electricity generation & 6.3 & 0.2 & 2.3 & 6.7 & 0.1 & 0.1 & 5.3 & 4.1 & -100.0 & -12.3 & 2.3 & 2.5 \\
\hline Coke production & 64.9 & -98.8 & 0.6 & & & & NS & -21.0 & & 0.3 & -6.3 & 1.8 \\
\hline Machine drive & & & 5.2 & 1.0 & 0.1 & 0.1 & 1.7 & 1.5 & 46.4 & 34.0 & & 5.0 \\
\hline Electrolyte processes & & & & & & & & 51.8 & 15.7 & & 1.9 & \\
\hline Other uses, MSK/NEC & 7.2 & 6.9 & 38.9 & 16.0 & & 5.4 & 23.7 & -7.6 & 0.1 & 42.5 & 41.4 & 21.4 \\
\hline Total & 100.0 & -1.2 & 100.0 & 100.0 & 100.0 & 100.0 & 100.0 & 79.4 & 0.0 & 87.7 & 93.7 & 100.0 \\
\hline
\end{tabular}


TABLE 3.3 Energy Purchases by Two-Digit Level Industries

\begin{tabular}{|c|c|c|c|c|c|}
\hline Rank & SIC & Industry & $\begin{array}{l}\text { Purchased Fuels } \\
\text { and Electricity } \\
\left(10^{12} \text { Btu/yr) }\right.\end{array}$ & $\begin{array}{r}\% \text { of } \\
\text { Total } \\
\end{array}$ & $\begin{array}{c}\text { Cumul at ive } \\
\frac{\%}{2}\end{array}$ \\
\hline 1 & 28 & Chemicals & 2716.7 & 22.9 & 22.9 \\
\hline 2 & 33 & Primary Metals & 2276.8 & 19.2 & 42.1 \\
\hline 3 & 26 & Paper \& Allied Products & 1278.4 & 10.8 & 52.8 \\
\hline 4 & 29 & Petroleum \& Coal & 1177.9 & 9.9 & 62.7 \\
\hline 5 & 32 & Stone, Clay \&lass & 1122.3 & 9.5 & 72.2 \\
\hline 6 & 20 & Food \& Kindred Products & 948.0 & 8.0 & 80.2 \\
\hline 7 & 34 & Fabricated Metal & 359.4 & 3.0 & 83.2 \\
\hline 8 & 37 & Transportation Equipment & 343.8 & 2.9 & 86.1 \\
\hline 9 & 35 & Machinery & 333.7 & 2.8 & 88.9 \\
\hline 10 & 22 & Textiles & 294.9 & 2.5 & 91.4 \\
\hline 11 & 36 & Electrical, Electronic & 239.9 & 2.0 & 93.4 \\
\hline 12 & 30 & Rubber, Miscellaneous Plastics & 223.0 & 1.9 & 95.3 \\
\hline 13 & 24 & Lumber \& Wood & 199.3 & 1.7 & 97.0 \\
\hline 14 & 27 & Printing \& Publishing & 88.2 & 0.7 & 97.7 \\
\hline 15 & 38 & Instruments & 79.7 & 0.7 & 98.4 \\
\hline 16 & 23 & Apparel & 58.2 & 0.5 & 98.9 \\
\hline 17 & 25 & Furniture \& Fixtures & 47.1 & 0.4 & 99.3 \\
\hline 18 & 39 & Miscellaneous & 45.0 & 0.4 & 99.7 \\
\hline 19 & 21 & Tobacco & 22.4 & 0.2 & 99.8 \\
\hline \multirow[t]{2}{*}{20} & 31 & Leather & 19.0 & 0.2 & 100.0 \\
\hline & & & $11,873.9$ & & \\
\hline
\end{tabular}

energy by industry at the four-digit SIC level for the top 80 energy-purchasing industries.

Individual Industry Information

An extensive literature review was conducted to determine the energy use characteristics of all two-digit SIC industries. Manufacturing processes, heattransfer operations, and waste heat stream characteristics of six industries are described in detail in Appendices $A$ through $F$. These industries are:

1) chemicals, 2) petroleum, 3) primary metals, 4) pulp and paper, 5) stone, clay and glass, and 6) textiles. These six industries account for almost $80 \%$ of the total energy used by the manufacturing sector. Table 3.5 shows the 
TABLE 3.4. Energy Purchases by the Top 80 Four-Digit Level Industries

\begin{tabular}{|c|c|c|c|c|c|}
\hline Rank & SIC & Industry & $\begin{array}{l}\text { Purchased Fuels } \\
\text { and Electricity } \\
\left(10^{12} \text { Btu/yr) }\right.\end{array}$ & $\begin{array}{l}\% \text { of } \\
\text { Total }\end{array}$ & Cumu lat ive \\
\hline 1 & 3312 & Blast Furnaces \& Steel Mills & 1282.1 & 10.8 & 10.8 \\
\hline 2 & 2911 & Petroleum Refining & 1098.5 & 9.3 & 20.0 \\
\hline 3 & 2869 & Industrial Organic Chemicals & 1005.9 & 8.5 & 28.5 \\
\hline 4 & 2621 & Papermilis & 583.0 & 4.9 & 33.4 \\
\hline 5 & 2631 & Paperboard Mills & 458.6 & 3.9 & 37.3 \\
\hline 6 & 3241 & Cement, Hydraulic & 390.8 & 3.3 & 40.6 \\
\hline 7 & 3334 & Primary Aluminum & 359.8 & 3.0 & 43.6 \\
\hline 8 & 2819 & Industrial, Inorganic Chemicals & 356.7 & 3.0 & 46.6 \\
\hline 9 & 2873 & Nitrogenous Fertilizers & 258.2 & 2.2 & 48.8 \\
\hline 10 & 2821 & Plastics Materials \& Resins & 187.3 & 1.6 & 50.4 \\
\hline 11 & 2865 & Cyclic Crudes \& Intermediates & 152.1 & 1.3 & 51.7 \\
\hline 12 & 3221 & Glass Containers & 132.0 & 1.1 & 52.8 \\
\hline 13 & 2824 & Organic Fibers, Noncellulosic & 127.4 & 1.1 & 53.9 \\
\hline 14 & 3079 & Misc. Plastics Products & 121.4 & 1.0 & 54.9 \\
\hline 15 & 3321 & Gray Iron Foundaries & 111.0 & 0.9 & 55.8 \\
\hline 16 & 3714 & Motor Vehicle Parts, Acc. & 110.9 & 0.9 & 56.7 \\
\hline 17 & 2812 & Alkalies and Chlorine & 100.2 & 0.8 & 57.5 \\
\hline 18 & 2611 & Pulpmills & 93.0 & 0.8 & 58.3 \\
\hline 19 & 2046 & Wet Corn Milling & 92.1 & 0.8 & 59.1 \\
\hline 20 & 3711 & Motor Vehicles \& Car Bodies & 89.7 & 0.8 & 59.9 \\
\hline 21 & 3274 & Lime & 85.6 & 0.7 & 60.6 \\
\hline 22 & 2063 & Beet Sugar & 71.7 & 0.6 & 61.2 \\
\hline 23 & 2011 & Meatpacking Plants & 69.6 & 0.6 & 61.8 \\
\hline 24 & 3353 & Aluminum Sheet, Plate \& Foil & 68.2 & 0.6 & 62.4 \\
\hline 25 & 3229 & Pressed and Blown Glass & 65.7 & 0.6 & 63.0 \\
\hline 26 & 2421 & Sawmills, Planing Mills & 59.2 & 0.5 & 63.5 \\
\hline 27 & 2813 & Industrial Gases & 58.1 & 0.5 & 64.0 \\
\hline 28 & 3251 & Brick \& Structural Clay Tile & 57.3 & 0.5 & 64.5 \\
\hline 29 & 2823 & Cellulosic Manmade Fibers & 56.6 & 0.5 & 65.0 \\
\hline 30 & 2874 & Phosphatic Fertilizers & 55.1 & 0.5 & 65.5 \\
\hline 31 & 3011 & Tires \& Inner Tubes & 54.0 & 0.5 & 66.0 \\
\hline 32 & 3296 & Mineral Wool & 53.9 & 0.5 & 66.5 \\
\hline 33 & 3331 & Primary Copper & 53.0 & 0.4 & 66.9 \\
\hline 34 & 2082 & Malt Beverages & 52.8 & 0.4 & 67.3 \\
\hline 35 & 3273 & Ready-Mix Concrete & 51.9 & 0.4 & 67.7 \\
\hline 36 & 3313 & Electrometallurgical Products & 51.0 & 0.4 & 68.1 \\
\hline 37 & 3211 & Flat Glass & 50.9 & 0.4 & 68.5 \\
\hline 38 & 2899 & Chemical Preparations, NEC & 49.3 & 0.4 & 68.9 \\
\hline
\end{tabular}


TABLE 3.4. (contd)

\begin{tabular}{|c|c|c|c|c|c|}
\hline Rank & SIC & Industry & $\begin{array}{l}\text { urchased Fuels } \\
\text { and Electricity } \\
\left(10^{12} \text { Btu/yr) }\right.\end{array}$ & $\begin{array}{r}\% \text { of } \\
\text { Total } \\
\end{array}$ & $\begin{array}{c}\text { Cumulat ive } \\
\end{array}$ \\
\hline 39 & 2075 & Soybean $0+1$ Mills & 48.0 & 0.4 & 69.3 \\
\hline 40 & 2221 & Weaving Mills, Manmade Fiber & 44.9 & 0.4 & 69.7 \\
\hline 41 & 2033 & Canned Fruits \& Vegetables & 44.7 & 0.4 & 70.1 \\
\hline 42 & 2822 & Synthetic Rubber & 42.6 & 0.4 & 70.5 \\
\hline 43 & 3275 & Gypsum Products & 42.5 & 0.4 & 70.9 \\
\hline 44 & 2816 & Inorganic Pigments & 41.2 & 0.3 & 71.2 \\
\hline 45 & 2051 & Bread, Cake, Related Products & 41.0 & 0.3 & 71.5 \\
\hline 46 & 2834 & Pharmaceutical Preparations & 40.0 & 0.3 & 71.8 \\
\hline 47 & 3462 & Iron \& Steel Forgings & 40.0 & 0.3 & 72.1 \\
\hline 48 & 3341 & Secondary Monferrous Metals & 39.3 & 0.3 & 72.4 \\
\hline 49 & 3531 & Construction Machinery & 37.6 & 0.3 & 72.7 \\
\hline 50 & 2653 & Corrugated Solid Fiber Boxes & 36.4 & 0.3 & 73.0 \\
\hline 51 & 2211 & Weaving Mills, Cotton & 35.9 & 0.3 & 73.3 \\
\hline 52 & 2951 & Paving Mixtures \& Blocks & 35.4 & 0.3 & 73.6 \\
\hline 53 & 3721 & Aircraft & 34.4 & 0.3 & 73.9 \\
\hline 54 & 2411 & Logging Camps, Log Contractors & 33.6 & 0.3 & 74.2 \\
\hline 55 & 2262 & Finishing Plants, Manmade Fiber & 33.3 & 0.3 & 74.5 \\
\hline 56 & 2026 & Fluid Milk & 32.9 & 0.3 & 74.8 \\
\hline 57 & 2099 & Food Preparations, NEC & 32.8 & 0.3 & 75.1 \\
\hline 58 & 2499 & Wood Products, NEC & 32.8 & 0.3 & 75.4 \\
\hline 59 & 3339 & Primary Nonferrous Metals, NEC & 32.8 & 0.3 & 75.7 \\
\hline 60 & 2062 & Cane Sugar Refining & 32.2 & 0.3 & 76.0 \\
\hline 61 & 2037 & Frozen Fruits \& Vegetables & 32.0 & 0.3 & 76.3 \\
\hline 62 & 2833 & Medicinals \& Botanicals & 31.4 & 0.3 & 76.6 \\
\hline 63 & 3523 & Farm Machinery \& Equipment & 30.3 & 0.3 & 76.9 \\
\hline 64 & 2077 & Animal \& Marine Fats \& 0ils & 28.9 & 0.2 & 77.1 \\
\hline 65 & 3069 & Fabricated Rubber Products, NEC & 28.4 & .0 .2 & 77.3 \\
\hline 66 & 2079 & Shortening \& Cooling 0ils & 28.0 & 0.2 & 77.5 \\
\hline 67 & 3361 & Aluminum Foundartes & 27.8 & 0.2 & 77.7 \\
\hline 68 & 3465 & Automotive Stampings & 27.7 & 0.2 & 77.9 \\
\hline 69 & 3295 & Minerals, Ground or Treated & 27.6 & 0.2 & 78.1 \\
\hline 70 & 3599 & Machinery, except Electrical, NEC & 27.5 & 0.2 & 78.3 \\
\hline 71 & 3411 & Metal Cans & 27.1 & 0.2 & 78.5 \\
\hline 72 & 2272 & Tufted Carpets \& Rugs & 26.8 & 0.2 & 78.2 \\
\hline 73 & 2661 & Building Paper \& Board Mills & 26.8 & 0.2 & 78.9 \\
\hline 74 & 2048 & Prepared Foods, MEC & 26.1 & 0.2 & 79.1 \\
\hline 75 & 2022 & Cheese, Natural \& Processed & 25.9 & 0.2 & 79.3 \\
\hline 76 & 2261 & Finishing Plants, Cotton & 25.9 & 0.2 & 79.5 \\
\hline
\end{tabular}


TABLE 3.4 (contd.)

\begin{tabular}{|c|c|c|c|c|c|}
\hline Rank & SIC & Industry & $\begin{array}{l}\text { Purchased Fuels } \\
\text { and } 1 \text { lectricity } \\
\left(10^{12} \text { Btu/yr) }\right.\end{array}$ & $\begin{array}{c}\% \text { of } \\
\text { Tota } 1 \\
\end{array}$ & $\begin{array}{c}\text { Cumulat ive } \\
\%\end{array}$ \\
\hline 77 & 3585 & Refrigeration, Heating Equipment & 25.5 & 0.2 & 79.7 \\
\hline 78 & 2879 & Agricultural Chemicals, NEC & 25.4 & 0.2 & 79.9 \\
\hline 79 & 2436 & Softwood Veneer \& Plywood & 24.7 & 0.2 & 80.1 \\
\hline 80 & 2752 & Conmercial Printing, Lithograph & 24.2 & 0.2 & 80.3 \\
\hline
\end{tabular}

Table 3.51982 Industrial Energy Consumption (adopted from DOE 1983)

\begin{tabular}{|c|c|c|c|}
\hline SIC & Industry & $\begin{array}{l}\text { Energy Use (a) } \\
\left(10^{1}{ }^{2} \text { Btu/yr) }\right.\end{array}$ & $\begin{array}{l}\% \text { of } \\
\text { Total } \\
\end{array}$ \\
\hline 28 & Chemicals & 5636.6 & 24.5 \\
\hline 29 & Petroleum & 5551.1 & 24.1 \\
\hline 33 & Primary metals & 3747.9 & 16.3 \\
\hline 26 & Pulp and paper & 1813.7 & 7.9 \\
\hline 32 & Stone, clay. and glass & 1148.1 & 5.0 \\
\hline \multirow[t]{3}{*}{22} & Textiles & 452.6 & 2.0 \\
\hline & Others & 4665.2 & 20.2 \\
\hline & Total & $23,015.2$ & 100.0 \\
\hline
\end{tabular}

(a) Includes captive fuels, purchased fuels, and fuels used to generate purchased electricity.

energy use for these six industries in 1982 on an absolute and percentage basis. These energy use figures include internally generated (captive) fuels, purchased fuels, and energy conservation losses in generating purchased electricity. Summaries of the waste heat stream information gathered for these six industries are provided in the following sections.

Chemicals

The chemical industry encompasses the manufacture of thousands of organic and inorganic chemicals by a variety of processes. The chemical industry is the largest user of energy in the manufacturing sector. Four 3-digit SIC product groups consume more than $90 \%$ of the energy consumed by the chemical 
industry: 1) SIC 281--Industrial Inorganic Chemicals, 2) SIC 282--Plastics and Synthetics, 3) SIC 286--Industrial Organic Chemicals, and 4) SIC 287-Agricultural Chemicals.

Table 3.6 lists the waste heat potential for the chemical industry by source. The waste heat stream information collected for specific chemical processes within the four top energy-consuming classifications is summarized below.

Industrial Inorganic Chemicals

The following industrial inorganic chemicals are examined in detail in the Appendix A:

- Chlorine

- Soda Ash

- Nitrogen

- Elemental phosphorus
- Caustic Soda

- Oxygen

- Sulfuric acid

- Phosphoric acid

Table 3.7 summarizes the waste heat temperatures and quantities for these chemicals. No significant waste heat streams were identified for the sulfuric acid

TABLE 3.6 Waste Heat Potential in the Chemical Industry $\left(10^{12} /\right.$ Btu/yr) (Nydick 1976)

\begin{tabular}{|c|c|c|c|c|c|}
\hline \multirow[b]{2}{*}{ Source } & \multicolumn{5}{|c|}{ Temperature ${ }^{\circ} \mathrm{F}$} \\
\hline & $\leq 150$ & $\begin{array}{l}150- \\
300 \\
\end{array}$ & $\begin{array}{l}300- \\
600 \\
\end{array}$ & $>600$ & Total \\
\hline Flue gas & $-\infty$ & -- & 350 & 60 & 410 \\
\hline Engine exhaust & - & $-\infty$ & 10 & 10 & 20 \\
\hline Waste steam & -- & -- & -- & -- & $-\infty$ \\
\hline $\begin{array}{l}\text { Gas cooling and } \\
\text { gas to liquid } \\
\text { condensation }\end{array}$ & 20 & 700 & 300 & 10 & 1030 \\
\hline $\begin{array}{l}\text { Solid products } \\
\text { and effluents }\end{array}$ & -- & 10 & $-\infty$ & 20 & 30 \\
\hline $\begin{array}{l}\text { Liquid products } \\
\text { and effluents }\end{array}$ & 120 & 190 & -- & -- & 310 \\
\hline Subtotal & 140 & 900 & 660 & 100 & 1800 \\
\hline $\begin{array}{l}\text { Radiation/convec- } \\
\text { tion/conduction } \\
\text { losses }\end{array}$ & - & & & & 200 \\
\hline Total heat releases & & & & & 2000 \\
\hline
\end{tabular}


TABLE 3.7 Waste Heat Stream Characteristics of Industrial Inorganic Chemical Processes

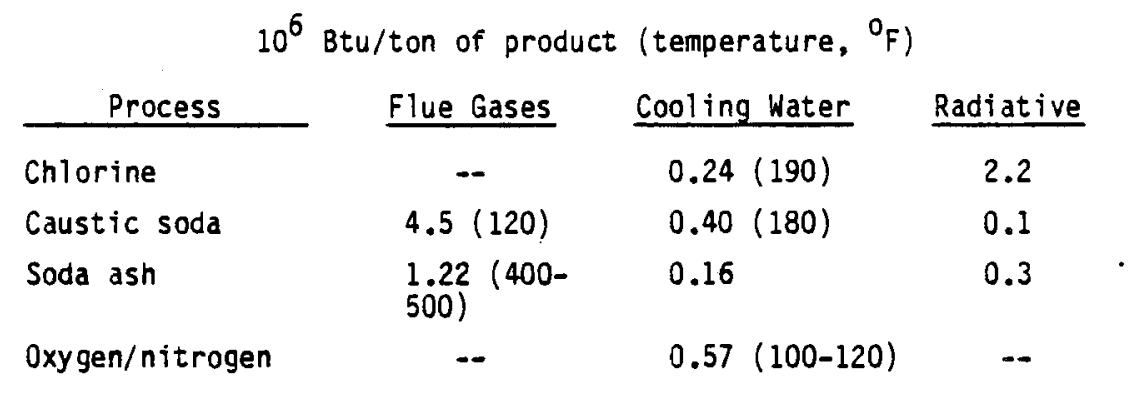

process. Two unit operations in this process are exothermic: sulfur burning and catalytic conversion of sulfur dioxide to sulfur trioxide. Waste heat is recovered from these operations to generate steam that is used either elsewhere in the plant or is exported. Little information was available on the waste heat from phosphorus and phosphoric acid production.

\section{Plastics and Synthetics}

The following plastics and synthetic materials were reviewed:
- low-density polyethylene
- polyester
- high-density polyethylene
- nylon
- polyvinyl chloride
- acrylic
- polystyrene

These processes are all characterized exothermic polymerization reactions, which may require high temperatures to initiate. Most of the process heat is provided in the form of steam and the condensate is returned to the boilers. The most important source of waste heat in these industries is cooling water, both contact and noncontact. Cooling water discharged from the high-density polyethylene process is the waste stream sufficiently large enough for waste heat recovery. The temperature of this cooling water typically ranges from 100 and $120^{\circ} \mathrm{F}$. The other source of waste heat is the flue gas from boilers used to generate process steam. These gases are at temperatures of about $400^{\circ} \mathrm{F}$. 


\section{Industrial Organics}

The following industrial organic chemicals are described in Appendix A:

$$
\begin{aligned}
& \text { - polyester } \\
& \text { - acrylic } \\
& \text { - phenol }
\end{aligned}
$$

- nylon

- styrene

- ethylene

The most significant process in terms of waste heat discharged is the styrene process. Between 0.6 and $0.7 \mathrm{million}$ Btu of waste heat is discharged per ton of styrene produced in flue gases at temperatures between 400 and $500^{\circ} \mathrm{F}$ (Latour 1981, UTC 1980). However, almost 50 times more waste heat is discharged in the cooling water from the styrene process, or $28.98 \mathrm{million} \mathrm{Btu}$ per ton of styrene at 100 to $120^{\circ} \mathrm{F}$ (Latour 1981). About $1.86 \mathrm{million}$ Btu per ton of ethylene are discharged in flue gases at temperatures around $400^{\circ} \mathrm{F}$ (Reding et al. 1975). The polyester process discharges flue gases in the range of 90 to $110^{\circ} \mathrm{F}$, although information on the quantity discharged was not available. Cooling water from polyester and nylon production ranges from 100 to $120^{\circ} \mathrm{F}$, again with no estimate of the quantities available.

\section{Nitrogenous Fertilizers}

The most significant chemical in this category is ammonia. This chemical accounts for most of the production of agricultural fertilizers and many of the remaining fertilizers are derived from this. Table 3.8 summarizes the waste

\begin{tabular}{|c|c|c|}
\hline Source & $\begin{array}{c}\text { Temperature } \\
\mathrm{F})\end{array}$ & $\begin{array}{l}\text { quantity } \\
\text { Btu/ton })\end{array}$ \\
\hline Reformer fiue gas & 400 & $2.4^{(a)}$ \\
\hline $\begin{array}{l}\text { Compressor cooling } \\
\text { water }\end{array}$ & $100-120$ & $0.5^{(\mathrm{a})}$ \\
\hline Boiler blowdown & $200^{(b)}$ & 0.03 \\
\hline Cooling tower blowdown & $20(b)$ & 0.2 \\
\hline Process condensate & $70^{(b)}$ & 0.2 \\
\hline
\end{tabular}
heat temperatures and quantities associated with ammonia production.

TABLE 3.8 Waste Heat Streams from Ammonia Production

(a) Estimate based on information for 1200 ton/day plant. (b) Temperature above ambient. 
Petroleum

There are only two significant sources of waste heat in a petroleum refinery: 1) hot flue gases from fired heaters, and 2) hot liquid product streams that are currently cooled to safe storage tempertures by water or air. Table 3.9 summarizes the sources and temperatures of these waste streams. Primary Metals

A significant amount of waste heat is rejected in the primary metals industries, both in flue gases and in waste cooling and process water. Estimates of the total waste heat rejected in flue gas as waste water for the top four energy-consuming industries are provided in Table 3.10. Almost half of

\section{TABLE 3.9 Sources of Refinery Waste Heat}

\begin{tabular}{lccc} 
Source & Temperature $\left({ }^{\circ} \mathrm{F}\right)$ & & $\begin{array}{c}10^{3} \text { Btu/bbl of } \\
\text { Oil Charge }\end{array}$ \\
\cline { 4 - 4 } $\begin{array}{llc}\text { Heater and boilerstacks } \\
\text { - Crude distillation }\end{array}$ & & $700-900^{\circ} \mathrm{F}$ & -- \\
- Catalytic reforming & $700-950^{\circ} \mathrm{F}$ & -- \\
- Coking & $800-1000^{\circ} \mathrm{F}$ & -- \\
- Hydrocracking & $700-900^{\circ} \mathrm{F}$ & -- \\
- Hydrogen production & $600-1000^{\circ} \mathrm{F}$ & -- \\
- Others & $300-1000^{\circ} \mathrm{F}$ & -- \\
Air coolers & $250-350^{\circ} \mathrm{F}$ & 174 \\
Water coolers & $150-250^{\circ} \mathrm{F}$ & 137
\end{tabular}

TABLE 3.10 Annual Waste Heat Discharged by Primary Metals Industries (Latour 1981)

\begin{tabular}{lc} 
& $\frac{10^{12} \text { Btu/yr }}{\text { Blast furnaces and steel mills }}$ \\
Gray iron foundaries & 1414.2 \\
Primary copper & 73.4 \\
Primary aluminum & 76.4 \\
\hline
\end{tabular}


the energy is rejected by these four processes is at temperatures less than $125^{\circ} \mathrm{F}$ (mostly cooling and process water). About $25 \%$ is at temperatures from 125 to $500^{\circ} \mathrm{F}, 20 \%$ is between 500 and $1000^{\circ} \mathrm{F}$, and the remainder is above $1000^{\circ} \mathrm{F}$.

Paper and Allied Products

Waste waters and saturated vapors at temperatures less than $350^{\circ} \mathrm{F}$ ae the most significant waste heat streams discharged from pulp mills (Labour 1981). Table 3.11 summarizes for pulp mills the types of waste heat sources and their heat content. The most significant sources of waste heat in paper and paperboard mills are 1) hot, moist air containing about $3.1 \times 10^{6}$ Btu/BDT, and 2) hot water containing about $0.69 \times 10^{6}$ Btu/BDT at about $250^{\circ} \mathrm{F}$. Stone, Clay and Glass

The major products in this classification are cement, glass (flat, container, pressed and blown), gypsum, brick and structural clay, mineral wool, and ready-mix concrete. With the exception of ready-mix concrete, the production process for each of these products is characterized by a high-temperature, direct-heat operation. Three of these industries, cement, lime, and glass

TABLE 3.11 Waste Heat Streams - Pulp Mills

(Latour 1981; UTC CTAS 1980;

Palmer et al. 1980)

\begin{tabular}{|c|c|c|}
\hline Source & Medium & $\begin{array}{c}\text { Reported } \\
\text { Theoretical } \\
\text { Heat Content } \\
\left(10^{6} \text { Btu/BDT }\right.\end{array}$ \\
\hline 1. Ground wood bleaching & Bleaching effluent & 1.1 \\
\hline 2. Digestor blowdown & Steam & 2.8 \\
\hline 3. Kraft bleaching & Bleach effluent & $0.4-1.2$ \\
\hline $\begin{array}{l}\text { 4. Multiple effect } \\
\text { evaporators }\end{array}$ & $\begin{array}{l}\text { Steam } \\
\text { Fou } 1 \text { condensate }\end{array}$ & $\begin{array}{l}3.3 \\
1.9\end{array}$ \\
\hline $\begin{array}{l}\text { 5. Recovery boilers } \\
\text { 6. Kiln }\end{array}$ & $\begin{array}{l}\text { Flue gases } \\
\text { Off gases }\end{array}$ & $\begin{array}{l}5.4 \\
0.82\end{array}$ \\
\hline
\end{tabular}

Sources: Latour 1981; UTC CTAS 1980; Paimer 1980. 
(including flat glass, container glass, pressed and blown glass, and mineral wool) consume $70 \%$ of the energy used by this industry classification. These three industries reject almost 0.4 quads $\left(393 \times 10^{12}\right.$ Btu) of waste heat each year in kiln and furnace exhausts (Latour 1981).

Sources and temperatures of waste heat are summarized in Table 3.12 and quantities of waste heat are summarized in Table 3.13 for cement, glass and lime.

Textiles

Sources and approximate temperatures of waste heat streams from textile operations are summarized in Table 3.14. A large portion of the energy is

TABLE 3.12 Sources and Temperatures of Waste Heat from Stone, Clay and Glass Industries

\begin{tabular}{|c|c|c|}
\hline Process & Source & Temperature $\left({ }^{\circ} \mathrm{F}\right)$ \\
\hline \multirow[t]{9}{*}{ Cement } & Kiln exhaust - wet & 150 \\
\hline & dry & 700 \\
\hline & preheater & $500-700$ \\
\hline & bypass & $1700-2000$ \\
\hline & Clinker cooler - wet & $150-250$ \\
\hline & dry & $300-450$ \\
\hline & preheater & $300-450$ \\
\hline & Finishing mill & 160 \\
\hline & Cooling water & $<100$ \\
\hline \multirow[t]{6}{*}{ Glass } & Melting furnace & $2000-2800$ \\
\hline & Primary regenerator & $1200-1500$ \\
\hline & Seconday regenerator & $800-1000$ \\
\hline & Metallic recuperator & $1600-1800$ \\
\hline & Annealing lehrs & $1000-1200$ \\
\hline & Cooling water & $<125$ \\
\hline \multirow[t]{3}{*}{ Lime } & Kfln exhaust & $1800-2000$ \\
\hline & Preheater exhaust & $450-550$ \\
\hline & Cooling water & $<100$ \\
\hline \multirow[t]{2}{*}{ Gypsum } & Calcining kettles & $700-1100$ \\
\hline & Dryers & $<200$ \\
\hline Brick \& clay & Kilns & $300-400$ \\
\hline
\end{tabular}


TABLE 3.13 Quantity of Waste Heat Discharged from Cement, Lime and Glass Production

\begin{tabular}{lc} 
& $10^{6}$ Btu/ton \\
\cline { 2 - 2 } Cement & \\
Kiln exit gases & 2.40 \\
Clinker cooling air & 0.54 \\
Cooling water & 0.05 \\
Shell losses & 0.90 \\
Glass & \\
Melter exit gases & 1.40 \\
Wall losses & 3.40 \\
Lime $\quad$ \\
Kiln exit gases \\
Product cooling air
\end{tabular}

TABLE 3.14 Sources of Waste Heat from Textile Mill Operations

\begin{tabular}{|c|c|c|}
\hline Type & Source & $\frac{\text { Temperature }}{(\mathrm{F})}$ \\
\hline \multirow[t]{3}{*}{ Exhaust gas } & Boilers & $400-600$ \\
\hline & Singeing ovens & $390-400$ \\
\hline & Tenter-frame aryers & $250-400$ \\
\hline Process water & $\begin{array}{l}\text { Slashing, oleaching, } \\
\text { washing, mercerizing, } \\
\text { dyeing }\end{array}$ & $100-120$ \\
\hline $\begin{array}{l}\text { Non-contact cooling } \\
\text { water }\end{array}$ & Product cooling & 140 \\
\hline
\end{tabular}

rejected from batch operations, such as drying. Exhausts from fabric ovens and dryers contain moisture, lint and finishing chemicals.

DATA BASE REVIEW

Information characterizing the industrial sector has been collected for years through censuses and surveys of manufacturers. Annual surveys of the fuels and electric energy purchased by industry are conducted by the U.S. Department of Commerce, Bureau of Census. Thus, considerable information is 
TABLE 3.15. Twenty-Five Industrial Energy Data Bases and Summary of SERI Report Findings (SERI 1980)

1. Federal Energy Administration (FEA) Energy Conservation Data Base for nine industries, developed by Gordian Associates, Inc. (1975)

2. Dow Chemical Company's Survey of Industrial Energy Use (1977)

3. DOE's End-Use Energy Consumption Data Base (ECDB) (1978b)

4. The data base associated with the Industrial Sector Technology Use Model (ISTUM)(Energy and Environmental Analyses, Inc. 1978)

5. The Industrial Process Energy Data Base of the Drexel University Industrial Applications Study (Hamel et al., 1979)

6. The Industrial Plant, Energy Profiles (IPEP) Data Base developed by General Energy Associates (1979)--a derivative of the Drexel data base

7. The Facility Energy Utilization Data System (FEUDS) developed by Ultrasystems, Inc. (undated)

8. Industrial Process Energy Data Base developed by Intertechnology Corporation (ITC 1977)

9. Industrial Process Heat Data Base developed by Battelle Columbus Laboratories (1977)

10. The Industrial Process Heat Data Base developed by SERI under the Industrial Energy Data Collection Cooperative effort (Green 1979)

11. Ongoing efforts at the Oak Ridge National Laboratory to develop a data base on industrial steam use (Barnes 1980)

12. The Environmental Protection Agency's (EPA) National Emissions Data System (1976)

13. Characterization of industrial process energy services by the Institute for Energy Analysis of Oak Ridge Associated Universities (1979)

14. Energy price distribution studies performed by Gruman Energy Data Systems (1979)

15. DOE's Price Data Base (1978) as part of the Federal Energy Data System

16. DOE's Major Fuel-Burning Installations (MFBI) Data Base (1975)

17. The Boiler Data Base available from the American Boiler Manufactures Association (ABMA, 1979)

18. Current DOE efforts to conduct a large-scale survey of industrial plants to obtain detailed end-use information (Wotecki 1980)

19. Other special studies resulting in data bases:

- Studies of industrial cogeneration by the Rocket Research Company (1978) for the Pacific Northwest

- Studies of industrial energy consumption in Missouri by Synergic Resources Corporation (1980c)

- Studies by Thermo Electron Corporation (1976) of industrial cogeneration

- Study by Resource Planning Associates (RPA, 1977) of industrial cogeneration for DOE

- Case studies of industrial cogeneration conducted by Synergic Resources Corporation (1980a) for the Electric Power Research Institute (EPRI)

- The industrial energy reporting system at DOE 
TABLE 3.15. (contd)

Major Findings

- Many data bases on industrial energy end use exist, but generally, the information reported was inconsistent.

- With the exception of ITC's and Battelle Laboratories' data bases, which were developed specifically to study solar industrial applications, none provide the information required for evaluating solar energy.

- Most data bases that report end-use information by quality and quantity of energy used represent only hypothetical or reference plants.

- Most data bases that have real plant data do not provide the detail required on the quality and quantity of energy end uses.

- No single data base can be considered uniformly better than any other.

- The Drexel University Industrial Process Energy Data Base, which details data on 108 industrial processes and uses the ITC and Battelle Information, is the most detailed data source for end uses at the 4-digit SIC level.

- The data bases vary significantly, perhaps corresponding to the variant energy consumption patterns throughout the U.S. Unfortunately, no attempt has been made to provide statistical measures of the actual variation in existing plants.

- Although considerable time and resources have been expended in developing the data bases, little effort has been devoted to verifying or validating the data.

- A considerable gap exists between what these data bases claim to provide and what they actualiy provide.

- A significant need exists for site-specific energy consumption data that can be used to develop realistic case studies on industrial solar energy applications. DOE is conducting a large-scale survey to develop such a data base, but it probably will not be available for several years. 
available on purchased energy inputs to manufacturing. However, for less information is available describing the flow of purchased and self-generated energy within industrial processes. Chapter 2.0 briefly discussed the problems of collecting energy flow data at the plant level and the complexity of the problem because of the diversity of industrial processes. This section reviews the work of others to establish industry energy data bases.

In recent years a number of independent studies have been conducted on the use of energy in industry. Many of the data bases associated with these studies have been reviewed by Synergic Resources Corporation (SRC) in conjunction with Solar Energy Research Institute (SERI) and for the Electric Power Research Institute (EPRI). The SERI/SRC evaluation (SERI/SRC 1980) investigated the application of several industrial energy data bases to obtain information for 1) industrial process heat market assessments, and 2) comparative evaluation of solar thermal systems for industrial process heat applications. A similar study conducted for EPRI (SRC 1982) focused on the needs of the electrical utility industry for information addressing the following items:

- end-use data

- type and size of loads

- technological changes

- time of day

- process demand profiles

- fuel substitution opportunities

- electricity conservation and cogeneration potential

- load management

- economic data.

The focus of these two reviews of industrial energy data bases are somewhat different from the focus for this report, which is to identify opportunities for using waste heat recovery technology. However, their discussion of the strengths and weaknesses of various data bases is applicable to this study. The SERI/SRC (1980) report identifies 24 data bases. These data bases and the major findings of the review are identified in Table 3.15. In the 
study two years later for EPRI, SRC (1982) reviewed in detail 11 major energy consumption data bases and concluded that none provided energy data in a form that would satisfy all the requirements of utilities. Table 3.16 identifies the 11 data bases reviewed in that study and the major findings (SRC 1982).

Each of these data bases were found by SRC to have significant weaknesses. Generally, they found that verification of data is a weakness of all data bases and that data bases were limited in providing detailed plant specific and geographic information.

From our review and SRC's, PNL determined that the Drexel University Industrial Process Energy Data Base had the best overall design capacity to handle the project needs. This data base is discussed in more detail in the next chapter. 
TABLE 3.16. Eleven Industrial Energy Data Bases and Summary of EPRI Report Findings (SRC 1982)

\section{Industrial Energy Data Bases}

1. The Potential for Energy Conservation in Nine Selected Industries: The Data Base, prepared for the Federal Energy Administration by Gordian Associates, June 1974.

2. Evaluation of the Theoretical Potential in Energy Conservation in Seven Basic Industries, prepared for the Federal Energy Administration by Battelle Columbus Laboratories, July 1975.

3. Energy Consumption: Fuel Utilization and Conservation in Industry, prepared for the Environmental Protection Agency by Dow Chemical Company, September 1975.

4. Environmental Considerations of Selected Energy Conservation Manufacturing Process Options, 15 volumes prepared for the Environmental Protection Agency by Aruthur'D. Little, Inc., December 1976.

5. Survey of the Applications of Solar Energy Systems to Industrial Process Heat, Final Report, prepared for the Energy Research and Development Administration by Battelle Columbus Laboratories, January 1977.

6. Analysis of the Economic Potential of Solar Thermal Energy to Provide Industrial Process Heat, Final Report, prepared for the Energy Research and Development Administration by InterTechnology Corporation (ITC), February 1977.

7. Energy Consumption Data Base, prepared for the Federal Energy Administration by Energy and Environmental Analysis (EEA), June 1977.

8. Energy Analysis of 108 Industrial Processes, prepared by Drexel University for the U.S. Department of Energy, June 1979.

9. Cogeneration Technology Alternative Study (CTAS), Volume II, Industrial Process Characteristics, Final Report, prepared for the U.S. Department of Energy NASA by United Technologies Corporation, January 1980.

10. Industrial Energy Data Book, prepared by Oak Ridge Associated Universities (ORAU) for the U.S. Department of Energy.

11. Industrial Plant Energy Profiles (IPEP), a proprietary data base developed by General Energy Associates (GEA).

\section{Major Findings}

- The data bases were not desgined to fulfill utility requirements for detail concerning electrical end uses--in fact, many of the data bases tend to ignore electrical end uses.

- Generally the data bases were developed to provide input for studies or models. This required that a wide range of data for plants with different characteristics be reduced to values for a "representative plant" because of the significant plant-to-plant variation within an industry. Such data are not useful when analyzing specific utility service areas. 



\subsection{APPLICATION OF SELECTED DATA BASE}

This chapter discusses the data base used in our analysis. Several steps were followed to implement an industrial energy-flow data base:

- After review of many data bases (Chapter 3.0), Drexel University's Industrial Process Energy Data Base was selected.

- The Drexel data base was updated from a 1979 data tape to incorporate 1980 data-base information.

- The Drexel data base was expanded to account for annual industry production.

- Calculated energy content values were adjusted to account for obvious limitations.

Our review and SRC's review of industrial energy data bases indicate that the data base with the best overall design capacity for handiing the information needs of this waste heat recovery assessment is the Drexel University Industrial Process Energy Data Base. This data base's design at the unit operation level gives it the capacity to handle detailed energy flow information more comprehensively than other data bases. For example, the data base allows for collection of unit operation temperature, inlet and outlet stream flow temperature, and mass and energy content data. Figure 4.1 shows a blowup of this information for a unit operation from the process flow diagram shown in Figure 2.1 of Chapter 2.0.

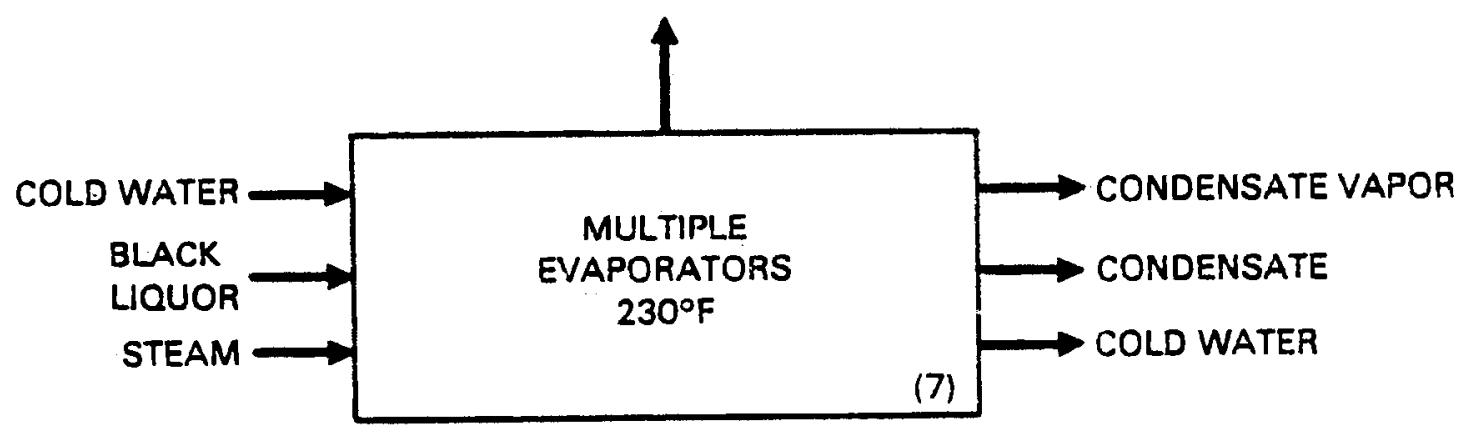

FIGURE 4.1 Evaporator Operation 
The Drexel data base includes typical process flow configurations for 108 industrial processes. These processes account for approximately $75 \%$ of U.S. industry purchased energy and fall within 61 four-digit SIC industries and 13 two-digit SIC industrial groups.

The 108 process flow configurations used in the Drexel data base are for typical plant operations for each process. The typical plant configurations developed are reported to be based on available references (engineering texts and handbooks, studies performed by private organizations and government agencies), limited on-site visits and consultant information (SERI/SRC 1980, p. 12). These data sources were used to develop heat and mass flow data on a per-poundof-product basis for each unit operation in an industrial process. Because the Drexel data base is designed to handle detailed data at the unit operation level, it is the most capable data base to address waste heat recovery source and sink questions.

Generally, the design of the Drexel data base matches well with the assessment needs of this study. The main criticism of the Drexel data base is that the data-base values are not documented. Review of Drexel data-base information indicates that it is not possible to determine the sources used to develop energy and mass balances for particular industries and that it is difficult to determine implicit assumptions contained in the data.

SRC's review of the Drexel data was based on three sources: Hamel et al. 1979; Hedman and Brown 1980; and 1980 computer printouts of unit operation flow charts. As part of their review, SRC evaluated one process and noted several questions that could not be resolved. In their overall review of the Drexel data base, SRC identified four limitations: 1) only hypothetical or reference plants are represented without any variation in energy-use patterns by geographic region or by plant size; 2) no systematic validation has been performed; 3) some relevant 4-digit groups are not covered; and 4) the quality and reliability of information used to compile the data vary significantly. Despite these limitations, SRC concluded that the end-use data provided by the Drexel can be valuable, particularly if some primary data are available to compare, to validate, or to modify the typical plant data. SRC also concluded that the data base represents the most detailed end-use data available on industrial energy 
consumption and is the only data base that provides information for a large number of 4-digit SIC industries.

Since the SRC review, Drexel University has published an additional document that considerably changes several industry process flow diagrams and changes many industry stream flow values (Drexel University 1980). These changes are discussed in some detail later. Although this latest report does provide considerable changes to the data in the Drexel data base, it still does not provide the additional documentation required. As a result, the Drexel data base as presently documented still has significant limitations placed on its usefulness.

Our review of the Drexel data base and its limitations indicates that the data base is not adequate for engineering level detail analysis nor for industry detailed analysis. The data base is most useful in assessing the general extent to which waste heat streams exit, their expected temperature ranges, and areas where recovered energy from waste heat streams may be used. Because this study's needs were for planning information, the Drexel data base was considered appropriate. Therefore, the 1979 data tape was placed on a System 2000 data base management system for review and updating. Once the data were updated, information for all 108 industrial processes was compiled. The data base was then expanded to calculate energy flow information over a one-year period and, where necessary, adjustments to these values were made.

\section{DATA BASE FILES}

Under contract with DOE, Drexel University had developed the industrial energy data base and placed the data base on computer tapes. That issue of the Drexel data base is herein referred to as the 1979 Drexel data base. The data tape as obtained from DOE came without instructions. Drexel University and others familiar with the Drexel data base were contacted for information about. operating the data base. The information we were able to obtain was helpful but was not complete. We then spent considerable time in developing a program to read the 1979 data tape and in implementing a data base management system. Once the data base became operational, the 1979 data tape and the 1980 edition of the data base were compared, and the 1979 data base was upgraded. 
Updating Drexel Industrial Data Files

The updated 1979 Drexel industrial data files are stored on computer disk files and backed up on magnetic tape on the UNIVAC 1100/44 computer at the Hanford site. The industrial data files consist of three types of records: General Industry Information Records, Unit Operation Records, and Flow Stream Records. Update changes were made to the Unit Operation Records and Flow Stream Records for each of the 108 five-digit SIC industries to make them agree with the 1980 Drexel documentation.

For the 14 industrial processes shown in Table 4.1, significant changes were made to the process flow diagram or to the energy flow values. For each of these processes, significant changes were made to the character of the process. For each of the 108 industrial processes, Table 4.2 identifies the kind of changes made to update the data tape.

TABLE 4.1 Process Industries With Significant Changes Between 1979 Tape and 1980 Drexel Manual

22 TEXTILE MILL PRODUCTS

2221-1 Weaving Mills, Man-Made Fibers

2262-1 Finishing Plants, Man-Made Fibers

24 LUMBER AND WOOD PRODUCTS

2411-1 Logging Camps, Log Contracts

2499-1 Wood Products, Fiberboard - Changed unit operation configuration

28 CHEMICALS

2812-1 Alkalies and Chlorine (Caustic Soda and Chlorine Diaphragm Cel1)

2822-2 Synthetic Rubbers (Buty1 Rubber)

2865-1 Cyclic Crudes and Intermediates (Styrene and Ethylbenzene)

2869-1 Industrial Organic Chemicals (Ethylene) - Changed unit operation configuration

2869-2 Industrial Organic Chemicals (Methanol) - Changed unit operation configuration

29 PETROLEUM

2911-1 Petroleum Refining

33 PRIMARY METALS

3312-1 Blast Furnaces and Steel Mills - Changed unit operation configuration

3313-1 Electrometallurgical Products

3331-1 Primary Copper

3334-1 Primary Aluminum 
TABLE 4.2. Changes Made to Update the 1979 Drexel Data Tape

20 FOOD AND KINDRED PRODUCTS

2011-1 Meat Packing Plants

Several changes to fuels used. Air conditioning and utility are deleted as separate unit operations.

2026-1 Fluid Milk

Several changes to fuels used. Space cooling and utility are deleted as separate unit operations.

2033-1 Canned Fuits and Vegetables (Canned Fruits)

Several changes to fuels used. Space cooling and utility are deleted as separate unit operations.

2033-2 Canned Fruits and Vegetables (Canned Vegetables)

Several label changes and changes to fuels used. Air conditioning and utility are deleted as separate unit operations.

2033-3 Canned Fruits and Vegetables (Canned Juices \& Drinks)

Several changes to fuels used. Air conditioning and utility are deleted as separate unit operations.

2046-1 Wet Com Milling

Several changes to fuels used. Space conditioning and utility are deleted as separate unit operations.

2051-1 Bread, Cake and Related Products (Bread)

Several changes to fuels used. Air conditioning and utility are deleted as separate unit operations.

2051-2 Bread, Cake, and Related Products (Cakes \& Pies)

Several changes to fuels used. Air conditioning and utility are deleted as separate unit operations.

2062-1 Cane Sugar Refining

Several label changes and changes to fuels used. Utility is deleted as a separate unit operation.

2063-1 Beet Sugar

Several changes to fuels used. Utility is deleted as a separate unit operation.

2075-1 Soybean $011 \mathrm{Mi} 11 \mathrm{~s}$

Seven unit operations are missing from the 1980 Manual on Page 47. Several changes to fuels used. Utility is deleted as a separate unit operation. 


\section{TABLE 4.2. (contd)}

\section{2-1 Malt Beverages}

Eight unit operations are missing from the 1980 Manual on Page 48. Several changes to fuels used. Utility is deleted as a separate unit operation.

\section{TEXTILE MILL PRODUCTS}

\section{1-1 Weaving Mi11s, Man-Made Fibers}

Massive numerical changes and label changes to many flow streams. Several changes to fuels used. Space heating, space cooling, and utility are deleted as separate unit operations.

\section{2-1 Finishing Plants Man-Made Fabrics}

Massive numerical changes to flow streams. Several changes to fuels used. Space heating, space cooling, and utility are deleted as separate unit operations.

24 LUMBER AND WOOD PRODUCTS

\section{1-1 Logging Camps, Log Contracts}

About one dozen numeric changes to flow streams. Several changes to fuels used. Utility is deleted as a separate unit operation.

2421-1 Saw Mills \& Planning Mills

One label change to flow stream name. Several changes to fuels used.

2499-1 Wood Products (Fiber Board)

Previously called Wood Products (Miscellaneous). Massive label and numerical changes to many unit operations and flow streams. Recode completely from 1980 Manual, Page 63.

\section{PAPER AND ALLIED PRODUCTS}

\section{1-1 Pulp Mills (Kraft Process)}

Minor label changes to flow stream names. Several changes to fuels used. Unit operations of space heating, space cooling, and utility are deleted as separate unit operations.

\section{1-2 Pulp Mills (Sulfite Process)}

Several minor label changes to flow stream names. Several changes to fuels used. Unit operations of space heating, space cooling, and utility are deleted as separate unit operations.

\section{1-3 Pulp Mills (Mechanical Process)}

Several dozen label changes to flow stream names. Several changes to fuels used. Unit operations of space heating, space cooling, and utility are deleted as separate unit operations.

2621-1 Paper Mills (Finishing Plant)

Several changes to fuels used. Unit operations of space heating, space cooling, and utility are deleted as separate unit operations. 


\section{TABLE 4.2. (contd)}

2621-2 Paper Mi11 (Integrated Mi11s)

Several changes to fuels used. Unit operations of space heating, space cooling, and utility are deleted as separate unit operations.

2631-1 Paperboard Mills (Finishing Plant)

Several changes to fuels used. Unit operations of space heating, space cooling, and utility are deleted as separate unit operations.

2631-2 Paperboard Mills (Integrated Plant)

Several changes to fuels used. Unit operations of space heating, space cooling, and utility are deleted as separate unit operations.

2653-1 Corrugated Solid Fiber Boxes

Several changes to fuels used. Unit operations of space cooling and utility are deleted as separate unit operations.

\section{1-1 Building Paper and Board Mills}

Several changes to fuels used. Unit operation of space heating, space cooling, and utility are deleted as separate unit operations.

2661-2 Building Paper \& Board Mills Integrated Plant

Several changes to fuels used. Unit operations of space heating, space cooling, and utility are deleted as separate unit operations.

28 CMEMICALS

2812-1 Alkalies and Chlorine (Caustic Soda and Chlorine-Diaphragm Cell)

Massive changes to numeric fields and labels of many unit operations and flow streams. Space heating, space cooling, and utility are deleted as separate unit operations.

2812-2 Alkalies and Chlorine ( $\mathrm{NAOH}, \mathrm{Cl}_{2}$--Mercury Process)

Card missing on tape for first flow stream (sait) for first unit operation -- Brine Preparation. Several changes to fueis used. Space heating, space cooling, and utility are deleted as separate unit operations.

2812-3 Alkalies and Chlorine (Soda Ash-Solvay Process)

Several changes to fuels used. Space heating, space cooling, and utility are deleted as separate unit operations.

2812-4 Alkalies and Chlorine (Soda Ash-Trona Deposits)

Several changes to fuels used. Space heating, space cooling, and utility are deleted as separate unit operatins.

2813-1 Inorganic Gases $\left(\mathrm{N}_{2}, \mathrm{O}_{2}\right)$

Space heating, space cooling, and utility are deleted as separate unit operations. 


\section{TABLE 4.2. (contd)}

2813-2 Inorganic Gases (H2-Steam Reform)

Several minor label changes and numeric changes to fuels used. Utility is deleted as a separate unit operation.

2813-2 Inorganic Gases (Acetylene)

Several changes to fuels used. Space heating, space cooling, and utility are deleted as separate unit operations.

2813-3 Inorganic Pigments ( $\mathrm{TiO}_{2}$-Chloride Process)

Several changes to fuels used. Space heating, space cooling, and utility are deleted as separate unit operations.

2816-1 Inorganic Pigments (TiO 2 -Sulfate Process)

Several changes to fuels used. Space heating, space cooling, and utility are deleted as separate unit operations.

2816-2 Inorganic Pigments (Zno-French Process)

Several changes to fuels used. Space heating and utility are deleted as separate unit operations.

2816-3 Inorganic Pigments (Zno-American Process)

One set of changes to fuels used. Space heating, space cooling, and utility are deleted as separate unit operations.

2816-5 Inorganic Figments (ZnO-Electrothermal Process)

One set of changes to fuels used. Space heating, space cooling and utility are deleted as separate unit operations.

2819-1 Irdustrial Inorganic Chemicals $\left(\mathrm{Al}_{2} \underline{\mathrm{O}}_{3}\right)$

Several changes to fuels used. Utility is deleted as a separate unit operation.

2819-2 Industrial Inorganic Chemicals (Sulfuric Acid)

Several label changes and utility is deleted as a separate unit operation.

2819-3 Industrial Inorganic Chemicals (Phosphorus)

Several changes to fuels used. Space heating, space cooling, and utility are deleted as separate unit operations.

2819-4 Industrial Inorganic Chemicals (Potash-Flotation Method)

Several changes to fuels used. Utility is deleted as a separate unit operation.

2819-5 Industrial Inorganic Chemicals (Sodium Metal)

Several changes to fuels used. Space cooling and utility are deleted as separate unit operations. 
TABLE 4.2. ( contd)

2821-1 Plastic Materials and Resins (PVC-Suspension Process)

Several changes to fuels used. Space heating, space cooling, and utility are deleted as separate unit operations.

2821-2 Plastic Materials and Resins (Polystyrene-Mass Process)

Several changes to fuels used. Space heating, space cooling, and utility are deleted as separate unit operations.

2821-3 Plastic Materials and Resins (LOPE)

Several changes to fuels used. Space heating, space cooling, and utility are deleted as separate unit operations.

28214 Plastic Materials and Resins (HDPE-Slurry Process)

Several changes to fuels used. Space heating, space cooling, and utility are deleted as separate unit operations.

2821-5 Plastic Materials and Resins (HDPE-Solution Process)

Several changes to fuels used. Space heating, space cooling, and utility are deleted as separate unit operations.

2822-1 Synthetic Rubbers (SBR)

Several changes to fuels used. Space heating, space cooling, and utility are deleted as separate unit operations.

2822-2 Synthetic Rubbers (Buty1 Rubber)

Several flow streams added to two unit operations. Several changes to fuels used. Space heating, space cooling, and utility are deleted as separate unit operations.

2822-3 Synthetic Rubbers (Polybutadiene Rubber)

Several changes to fuels used. Space heating, space cooling, and utility are deleted as separate unit operations.

2822-4 Synthetic Rubbers (Polyisoprene)

Several changes to fuels used. Space heating, space cooling, and utility are deleted as separate unit operations.

2822-5 Synthetic Rubbers (EP Rubber)

Several changes to fuels used. Space heating, space cooling, and utility are deleted as separate unit operations.

2823-1 Cellulosic Man-Made Fibers (Rayon)

Several changes to fuels used. Space heating, space cooling, and utility are deleted as separate unit operations. 
TABLE 4.2. (contd)

2823-2 Cellulosic Man-Made Fibers (Cellose Acetate)

Several label changes to flow streams and changes to fuels used. Space heating, space cooling, and utility are deleted as separate unit operations.

2824-1 Organic Fibers, Non-Cellulosic (Polyester)

Several changes to fuels used. Space heating, space cooling, and utility are deleted as separate unit operations.

2824-2 Organic Fibers, Non-Cellulosic (Polypropylene Fibers)

Several changes to fuels used. Space heating, space cooling, and utility are deleted as separate unit operations.

2824-3 Organic Fibers, Non-Cellulosic (Acrylics)

Several changes to fuels used. Space heating, space cooling, ard utility are deleted as separate unit operations.

2824-4 Organic Fibers, Non-Cellulosic (Nylon 6, 6)

Several changes to fuels used. Utility is deleted as a separate unit operation.

2824-5 Organic Fibers, Non-Cellulosic (Ny] on 6)

Several changes to fuels used. Space cooling and utility are deleted as separate unit operations.

2834-1 Pharmaceutical Preparations

Several changes to fuels used. Space heating, space cooling, and utility are deleted as separate unit operations.

2865-1 Cyclic Crudes and Intermediates (Styrene and Ethylbenzene)

Massive changes to numeric values for many unit operations and flow streams. Space heating and utility are deleted as separate unit operations.

2865-2 Cyclic Crudes and Intermediates (Cumene and Phenol)

Several changes to fuels used. Space heating, space cooling, and utility are deleted as separate unit operations.

2865-3 Cyclic Crudes and Intermediates (Cyclohexane)

Several changes to fuels used. Space heating, space cooling, and utility are deleted as separate unit operations.

2869-1 Industrial Organic Chemicals (Ethylene)

Massive label and numerical changes to many unit operations and flow streams. Recode completely from 1980 Manual, Pages 203-204. Space heating and utility are deleted as separate unit operations. 
TABLE 4.2. (contd)

2869-2 Industrial Organic Chemicals (Methanol)

Massive label and numerical changes to many unit operations and flow streams. Recode completely from 1980 Manual, Pages 206-207. Space heating, space cooling, and utility are deleted as separate unit operations.

2869-3 Industrial Organic Chemicals (Formaldehyde)

Several changes to fuels used. Space heating, space cooling, and utility are deleted as separate unit operations.

2873-1 Nitrogenous Fertilizers (Ammonia)

Several label changes to flow streams. Several changes to fuels used. Space heating, space cooling, and utility are deleted as separate unit operations.

2874-1 Phosphatic Fertilizers (Phosphoric Acid-Wet Process)

Several label changes to flow streams. Several changes to fuels used. Space heating, space cooling, and utility are deleted as separate unit operations.

2874-2 Phosphatic Fertilizers (Phosphoric Acid-Oxidation Method)

Several changes to fuels used. Space heating, space cooling, and utility are deleted as separate unit operations.

2874-3 Phosphatic Fertilizers (Superphosphates)

Several changes to labels of flow streams and to fuels used. Space heating, space cooling, and utility are deleted as separate unit operations.

2874-4 Phosphatic Fertilizers (Ammonium Phosphates)

Several changes to labels of flow streams and to fuels used.

Space heating, space cooling, and utility are deleted as separate unit operations.

2899-1 Chemical Preparations, NEC (Salt)

Several changes to fuels used. Space heating, space cooling, and utility are deleted as separate unit operations.

2899-2 Chemical Preparations, NEC (Fatty Acids-Continuous Process)

Several changes to fuels used. Space heating, space cooling, and utility are deleted as separate unit operations.

29 PETROLEUM

\section{1-1 Petroleum Refining}

Dozens of numeric changes and some label changes to flow streams. Utility is deleted as a separate unit operation. Several changes to fuels used.

2951-1 Paving Mixtures and Blocks

Several changes to fuels used. Space heating, space cooling, and utility are deleted as separate unit operations. 


\section{TABLE 4.2. (contd)}

30 RUBBER AND MISCELLANEOUS PLASTIC PRODUCTS

3011-1 Tires and Inner Tubes

Several changes to fuels used. Space cooling, space heating, and utility are deleted as separate unit operations.

3069-1 Fabricated Rubber Products, NEC (Dipped Latex)

Several label changes and changes to fuels used. Space cooling, space heating, and utility are deleted as separate unit operations.

3069-2 Fabricated Rubber Products, NEC (Molded Latex)

Several label changes and changes to fuels used. Space cooling, space heating, and utility are deleted as separate unit operations.

3069-3 Fabricated Rubber Products, NEC (Molded Products)

One label change and one change to fuels used. Space cooling and utility are deleted as separate unit operations.

3079-1 Miscellaneous Plastic Products

Several changes to fuels used. Space heating, space cooling, and utility are deleted as separate unit operations.

32 STONE, CLAY, AND GLASS

$3211-1 \quad$ Flat Glass

One numeric change to flow stream temperature. Several changes to fuels used. Space heating, space cooling, and utility are deleted as separate unit operations.

3221-1 Glass Contajners

Several changes to fuels used. Space heating, space cooling, and utility are deleted as separate unit operations.

3229-1 Pressed \& Blown Glass

Twenty-six minor label changes to flow stream names. Unit operations of space heating, space cooling, and utility are deleted. Several changes to fuels used.

3241-1 Cement, Hydraulic

Unit operations of space cooling, space heating, and utility are deleted. Several changes to fuels used.

3251-1 Brick and Structural Clay Tile

Fourteen minor label changes to flow stream names. Unit operations of space heating, space cooling, and utility are deleted. Several changes to fuels used.

3273-1 Ready Mix Cement

Five minor label changes to flow stream names. Unit operations of space cooling, space heating and utility are deleted. Several changes to fuels used. 
TABLE 4.2. (contd)

3274-1 Lime

Eighteen minor label changes to flow stream names. Unit operations of space heating, space cooling, and utility are deleted. Several changes to fuels used.

3275-1 Gypsum Products

Two minor label changes to flow stream names. Unit operations of space heating, space cooling, and utility are deleted. Several changes to fuels used.

3296-1 Mineral Wool

Three minor label changes to flow stream names. Unit operations of space heating, space cooling, and utility are deleted. Several changes to fuels used.

33 PRIMARY METALS

\section{2-1 Blast Furnaces and Steel Mills}

Massive label and numeric changes to many unit operations and flow streams. One dozen old unit operations are deleted. Redo completely from 1980 manual, Pages 268-272.

3313-1 Electrometallurgical Products

Several dozen changes to numeric data and labels of flow streams. Unit operations of space heating, space cooling, and utility are deleted. Several changes to fuels used.

3321-1 Gray Iron Foundries

Unit operation of utility is deleted. Several changes to fuels used.

3331-1 Primary Copper

Several dozen changes to numeric data and labels of flow streams. Unit operation of utility is deleted. Several changes to fuel used.

3334-1 Primary Aluminum

About one dozen changes to numeric data and labels of flow streams. Unit operation of utility is deleted. Several changes to fuels used.

3341-1 Secondary Non Ferrous Metal 5-Aluminum

Minor label changes to flow streams. Unit operation of utility is deleted. Several changes to fuels used.

3341-2 Secondary Non Ferrous Metals (Lead)

Minor changes to labels of flow streams. Utility operation is deleted. Several changes to fuels used. 


\section{TABLE 4.2. (contd)}

3341-3 Secondary Non Ferrous Metals (Zinc)

Minor changes to labels of flow streams. Utility operation is deleted. Several changes to fuels used.

3341-4 Secondary Non Ferrous Metals (Copper)

Utility operation is deleted. Several changes to fuels used.

3353-1 Aluminum Sheet Plate Foil

Minor label changes to flow streams. Utility operation is deleted. Several changes to fuels used.

\section{FABRICATED METALS}

3462-1 Iron and Steel Forging

Several changes to fuels used. Space heating, space cooling, and utility are deleted as separate unit operations.

35 MACHINERY, EXCEPT ELECTRICAL

3523-1 Farm Machinery and Equipment

Several changes to fuels used. Space heating, space cooling, and utility are deleted as separate unit operations.

3531-1 Construction Machinery

Several changes to fuels used. Utility is deleted as a separate unit operation.

37 TRANSPORTATION EQUIPMENT

3711-1 Motor Vehicles and Car Bodies

Several changes to fuels used. Space heating, space cooling, and utility are deleted as separate unit operations.

3714-1 Motor Vehicle Parts and Accessories

Several changes to fuels used. Space heating, space cooling, and utility are deleted as separate unit operations.

38 INSTRUMENTS AND RELATED PRODUCTS

3861-1 Photographic Film

Several changes to fuels used. Space cooling, space heating, and utility are deleted as separate unit operations.

3861-2 Pnotographic Equipment

Several changes to fuels used. Space heating, space cooling, and utility are deleted as separate unit operations. 
Record Layout

The record layout for the Unit Operation Records in the Drexel industrial data files was slightly revised to incorporate a new data element called "Total Fuels Used." Figure 4.2 shows the revised record layout for the Drexel industrial data files.

The data elements are defined in Table 4.3 and the codes for specific elements are shown in Table 4.4. The new data element, "Total Fuels Used," was inserted into the third card-image data record of the Unit Operation Records in columns 51-60. The rationale for adding the new data element is discussed below.

Research analysts at Drexel University originally itemized fuel consumption in each of the five-digit SIC process codes by five categories: coal, distilled oil, residual oil, natural gas, and other fuels. However, it was noticed that the consumption of natural gas, distilled oil, and residual oil always followed one of two ratios:

1. $50 \%$ by natural gas, $25 \%$ by distilled $0 i 1$, and $25 \%$ by residual oil, or 2. $75 \%$ by natural gas, $12.5 \%$ by distilled 011 , and $12.5 \%$ by residual $0 i 1$. No documentation was provided by Drexel University to justify this "assumed" pattern of fuel consumption for natural gas, distilled oil, and residual oil across the 108 five-digit codes. Consequently, the updated files (as well as the 1980 Drexel report) provide only a total fuel consumption value for each industry. Energy consumption is no longer reported by subtotals for natural gas, distilled oil, residual oil, coal and other fuels; however, the electrical consumption and unrecoverable losses are still reported separately in the updated files. This format follows the 1980 Drexel document.

- Space heating, space cooling, and utility operations were also dropped as separate unit operations in the updated files to conform to the 1980 Drexel document. The boiler operation and electric generation operation were retained, both in the 1980 Drexel document and the updated files. 


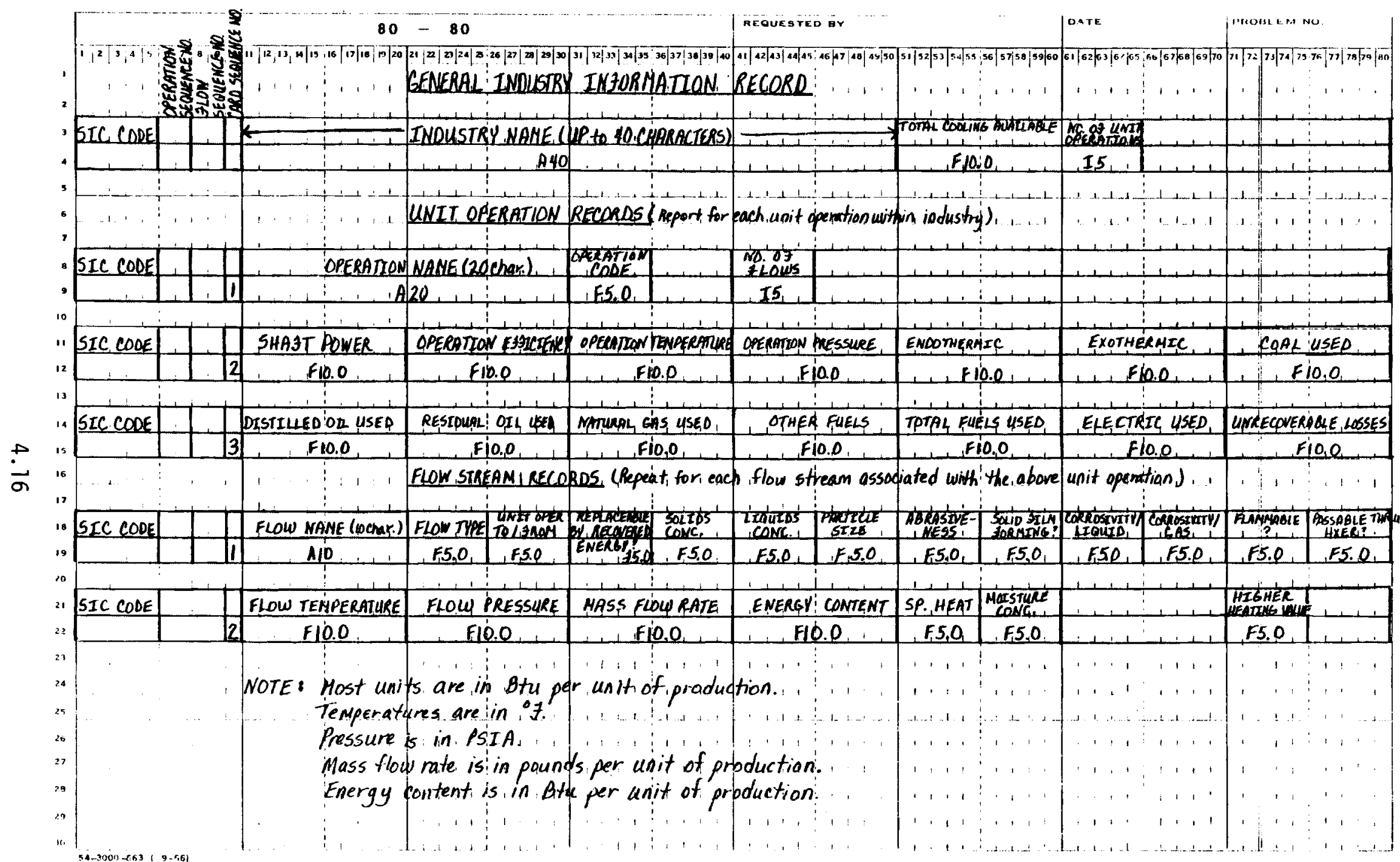

FIGURE 4.2 Record Layout for Drexel Industrial Data Files Loaded into System 2000 DBMS 


\section{TABLE 4.3 Definition of Drexel Data Elements}

General Industry Information Record--Description of Inputs

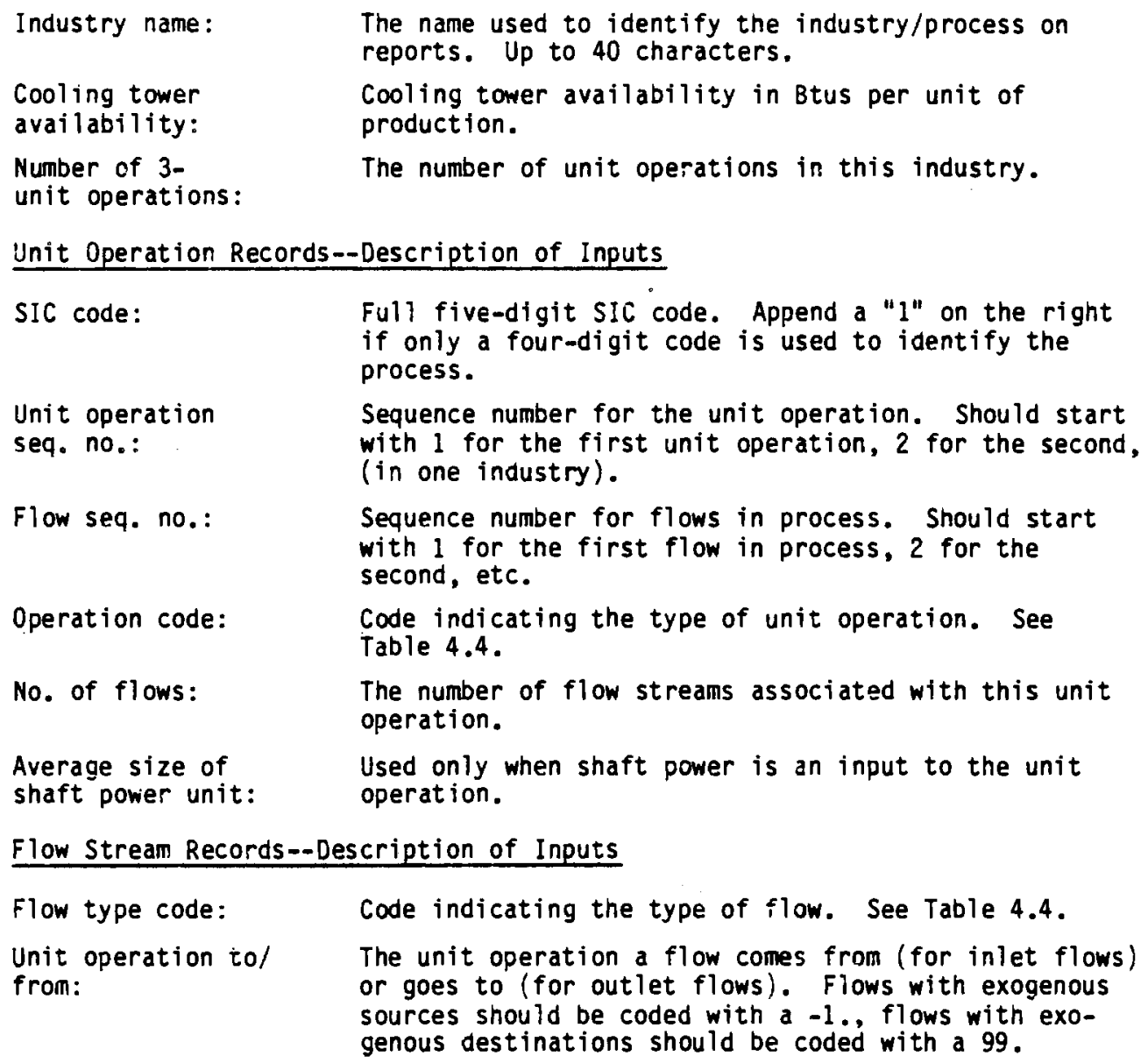

The updated Drexel industrial data files for the 108 five-digit codes were then loaded into the System 2000 Data Base Management System (DBMS) on the UNIVAC 1100/44 computer at the Hanford site. This data base was used for on1ine query, updating, and report generation by using System 2000 DBMS commands at our computer terminals. The definition of the records and data elements ("schema") for the resulting System 2000 data base is shown in Table 4.5 . Simple English-like commands, described in the next subsection, were used to query, sort, select, and update data from the System 2000 data base. 


\section{TABLE 4.4 Drexel Data Element Codes (a)}

Unit Operation Code

Mechanical $(1-19)$

1. Compressor

2. Refrigeration

10. Centrifuge

3. Mixing

4. Crushing, grinding

11. Pumps

5. Separation

12. Major mech. proc. (misc.)

6. Filter

13. Packaging

7. Extruding

14. Filling

8. Rolling

9. Cutting, triming

15. Settling

16. Spinning, weaving

17. Storage, holding tank

18. Misc.

Therma 1 (20-40)

20. Furnace

21. Drying

31. Condenser

22. Cooking

32. Distillation

23. Ovens

33. Flash separator

34. Prime mover, elect.

24. Washing

25. Evaporation

35. Prime mover, mech.

36. Turbine

26. Annealing

37. Cooling tower

27. Pasteurizing

38. Cooling

28. Casting, molding

39. Heating

29. Boiler

40. Misc.

30. Heat exchangers

Thermal-Chemical (41-49)
41. Reactors
42. Coking
43. Electrolytic cells
44. Extraction

45. Absorbing

46. Stripping

47. Leaching

48. Crystallization

Misc. (50-59)
50. Feedstocks
51. Transportation
52. Lighting

53. Space heating

54. Space cooling

55. Electricity

56. Misc.

(a) Other data elements in Figure 3.2 are self-explanatory. 


\section{TABLE 4.4 (contd)}

Inlet Flows

1.0 Electrical

2.0 Shaft power

3.1 Process hot air

3.2 Process hot water

3.3 Process steam

4.1 Process cool air

4.2 Process cool water

6.0 Refrigeration

7.0 Space heating

8.0 Space cooling

9.1 Product gas

9.2 Product liquid

9.3 Product solid

9.4 Return Condensate

Stream Flow Characteristics
Outlet Flows

21.0 Waste gas

22.0 Waste water

23.0 Waste steam

24.0 Solid waste

25.0 Return condensate

26.0 Gas product

27.0 Liquid product

28.0 Solid product

29.0 Process steam

30.0 Electricity

31.0 Undefined losses

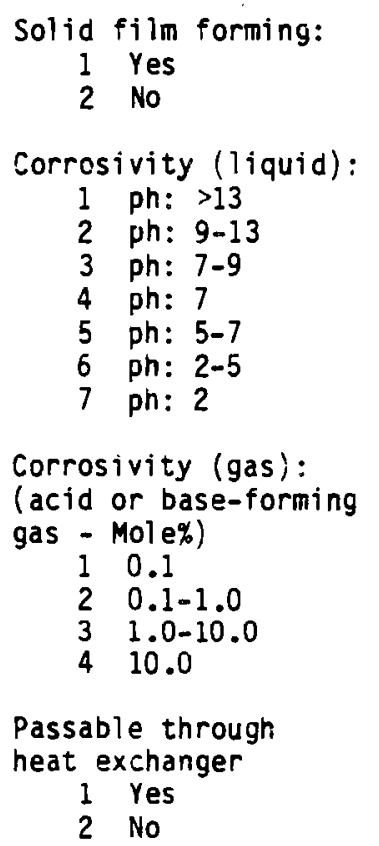


TABLE 4.5. Schema for Updated System 2000 Drexel Data Base

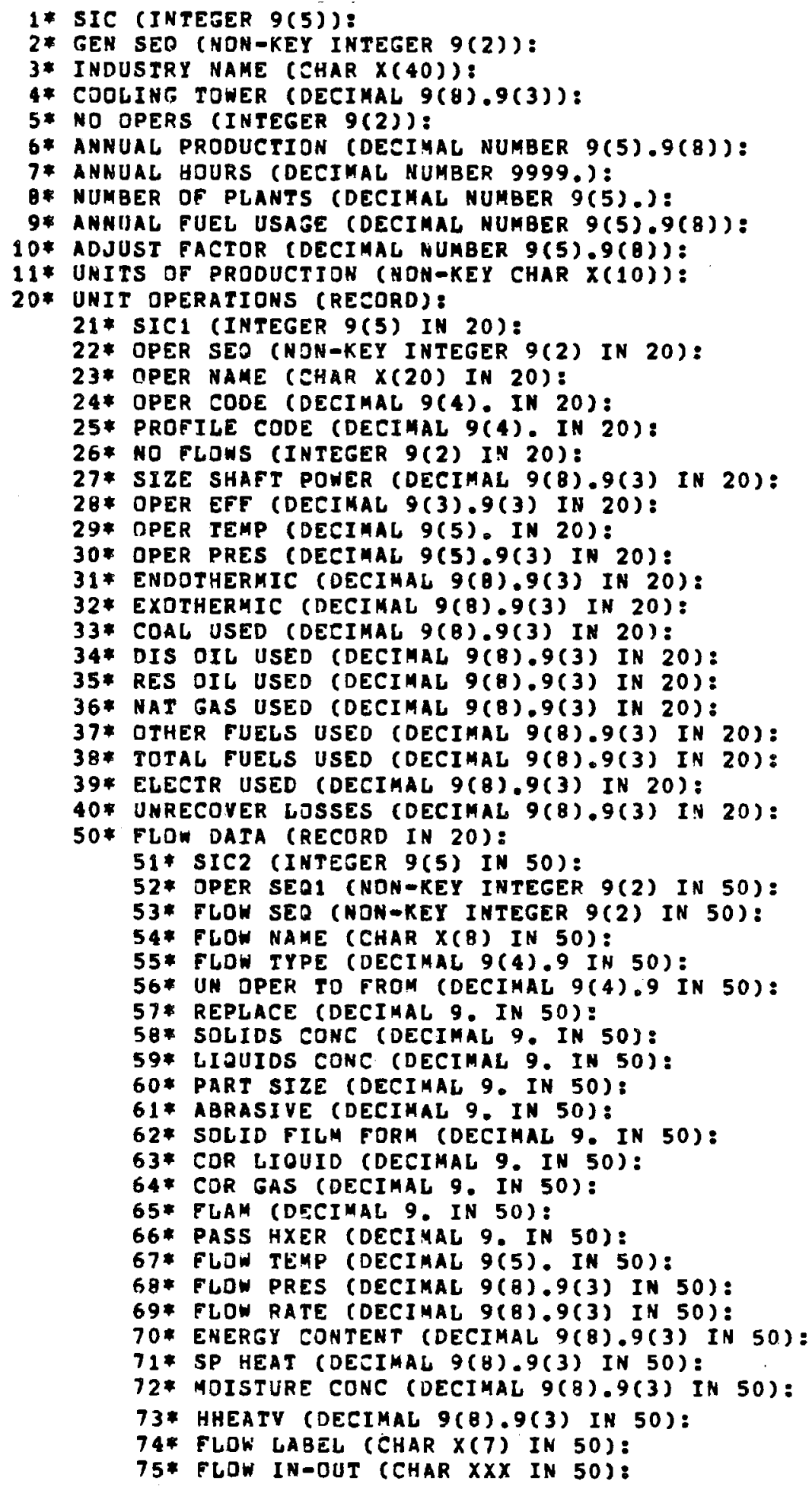


GENERAL CAPABILITIES OF SYSTEM 2000 DBMS

System 2000 is a Data Base Management System that was developed and is maintained by Intel Corporation in Austin, Texas. The system operates on IBM, Univac, Amdahl, and CDC large-scale computers. Most of the information on System 2000 described in this section was obtained from Intel's System 2000 Professional User's Guide.

System 2000 contains multiple modules, i.e., English-like command languages, to provide the user with commands to name, organize, load, archive, query, sort, update, and release data bases. Some of the key modules of System 2000 are shown in Table 4.6.

Users can dial into an on-1ine System 2000 data base located at a host computer site that has the System 2000 DBMS, such as the DOE headquarters or the Hanford site, through the FTS telephone network or through some other commercially available time-sharing network. Because the QUEST language is the module that users would most frequently use at their terminal to access information and to browse through a System 2000 data base, the remainder of the discussion in this subsection will focus on this module.

Commands in the QUEST 1 anguage are easy to learn and use. The most frequently used QUEST commands for data retrieval and data browsing are DESCRIBE, TALLY, PRINT, and LIST. Each of these commands is discussed briefly below.

DESCRIBE shows the user the data base definition. This includes the names of the data items, their data type (numeric or alphanumeric), their maximum number of anticipated digits or characters (their "picture"), the relationships among the data records, and various other attributes.

TALLY is used to count the distinct values of a data item, to determine the number of times each distinct value occurs in the data base, and to display the minimum and maximum values for each item.

PRINT will display requested data values. Either the entire data base or a subset of the data base can be scanned with this command.

LIST will produce columns of data with either default or user-specified column headings. Again, either the entire data base or a subset can be scanned. 
TABLE 4.6 Modules of System 2000

Module

DEFINE language

CONTROL language

QUEST (immediate

access) language

REPORT language

PLEX (programming

language extension)

Genius

Quex
Purpose

To specify the names of the data items, the types of data, and the relationships among the data records in a data base (i.e., the data base definition).

To save or to restore a data base and to provide security.

To load, query, retrieve, sort, and update a data base.

To generate reports.

To load and retrieve large volumes of data through a programing language such FORTRAN, COBOL, PL/1, or Assembler.

To generate reports and graphic charts through a series of conversational prompts and user responses from a terminal.

To enter and retrieve data interactively from teminal:

A wide variety of options are available with each of these four main commands. The user can select a subset of data items to be displayed by specifying a where-clause and selection criteria in the PRINT or LIST commands. The user can also sort the output by giving an ordering-clause whenever a whereclause is given in a PRINT or LIST command.

The following commands in the QUEST language can be used to update the data base:

$\underline{A D D}$ is used to add an item value where none currently exists. ASSIGN is used to assign a value to an existing data item. CHANGE is used to change an existing item value.

- REMOVE deletes an item value.

INSERT TREE inserts a new data tree of records and values. REMOVE TREE deletes a data tree of records and values.

Detailed information on each of these commands, including examples, and information on each of the above modules is available through the following publications by Intel: 
Language Specification Manuals

DEFINE language

QUEST language, including the QUEUE option

Programming Facility (PLEX)

Administrative Facilities, including CONTROL language

Messages and Codes (MAC)

CREATE facility

User's Guides

System 2000 Professional User's Guide

Genius

Quex

Pocket Guides

Syntex Guide

Glossary

DATA BASE EXPANSION/ADJUSTMENTS

At this point the data base was operational, and the energy flow information associated with the unit operations of selected industrial processes was specified in Btus per unit of production. To estimate the annual energy content embodied in these various heat streams, annual production information developed for a prior DOE report (G.E. 1981) was compared to information from the 1977 Census of Manufactures. The relationship among industrial processes characterized in the Drexel data base and the industries characterized in the Census of Manufactures is not always direct. Differences occurred in three areas:

1. The Drexel data base often divides SIC codes into subunits based on the different types of processes used to produce a product (i.e., three types of pulp mills), whereas the 1977 Census of Manufactures divides the SIC codes by types of goods--not processes. This makes it difficult to assign Census of Manufactures production levels to some Drexel processes.

2. In several cases, the production units used by Drexel and the Census of Manufactures were not the same. 
3. In some industries, Census of Manufactures estimates of annual production were unavailable or undisclosed.

Industry Production Information

The Drexel data base provides energy flow information in Btus per pound of product for typical industrial processes. To estimate the quantity of waste heat energy that a plant or industry might generate annually, industrial production information must be incorporated into the Drexel data base. Using G.E. and Census of Manufactures data, production values for each of the 108 processes characterized by the Drexel data base were compiled.

Efforts were made to independently relate the Drexel industrial processes to the Census of Manufactures product data. Where the Census of Manufactures data was used, the results are reported in Table 4.7. The first two columns of the table shows the Drexel data base code used for all 108 industrial processes and the production units used in describing stream flows. Column three reports the production units used in the Census of Manufactures. Column four provides the conversion factor for converting Drexel data base production unit to the production unit used in the Census of Manufactures. Column five identifies the 1977 estimates of production obtained from the Census of Manufactures and column six provides the multiplier used for converting Drexel data base stream flow information into annual data.

This assessment uses the G.E. values as the basic estimate of production and modifies them based on the 1977 Census of Manufactures data. The revised values are close to G.E.'s original values, except for processes 2262-1, 2951-1, and 3273-1. In the case of Process 2262-1, the conversion factor used to convert linear feet of material to pounds is corrected. For Process 2951-1 and 3273-1, differences appear to stem from different interpretations of Census of Manufactures information and possibly different conversion factors.

Table 4.8 compares the U.S. industrial production estimate used in this report to those used by G.E. 
TABLE 4.7 Industry Production Information for Use in
Converting Drexel Data to Annual Data

\begin{tabular}{|c|c|c|c|c|c|}
\hline $\begin{array}{l}\text { Drexel } \\
\text { Industry } \\
\text { Code } \\
\end{array}$ & $\begin{array}{l}\text { Drexel } \\
\text { Units of } \\
\text { Production } \\
\end{array}$ & $\begin{array}{c}\text { Census of } \\
\text { Menufacturers } \\
\text { Units of Production }\end{array}$ & $\begin{array}{l}\text { Conversion from } \\
\text { Drexel inits to } \\
\text { and) Units } \\
\end{array}$ & $\begin{array}{c}1977 \\
\text { Or (a) } \\
\text { Production }\end{array}$ & $\begin{array}{l}\text { Multipl ier for } \\
\text { Converting Drexel } \\
\text { Data to Annual Data }\end{array}$ \\
\hline $2011-1$ & Pounds & Milition pounds & $1,000,000$ & $31,675.1$ & $31,575 \times 10^{6}$ \\
\hline $2026-1$ & Pounds & Millian pands & $1,000,000$ & $15,816.4$ & \\
\hline $2026-1$ & Pands & Mllion quarts & $202025 \times 10^{6}$ & $22,065 \mathrm{~A}$ & \\
\hline 2026 total & Pounds & & & & $61,786 \times 10^{6}$ \\
\hline $2033-1$ & Case & 1,000 cases & 1,000 & $150 J 48$ & $151 \times 10^{6}$ \\
\hline $20033-2$ & Case & 1,000 cases & 1,000 & 251.127 & $251 \times 10^{6}$ \\
\hline $2033-3$ & Case & 1,000 cases & 1,000 & 157000 & $157 \times 10^{6}$ \\
\hline $2046-1$ & $\begin{array}{l}\text { Pand of } \\
\text { com input }\end{array}$ & Million pounds & $1,000,000$ & 15,180 & $15.18 \times 10^{9}$ \\
\hline $2051-1$ & 100 pands & Million pounds & 10,000 & 10,883 & $109 \times 10^{6}$ \\
\hline $2051-2$ & 100 pounds & Milion pounds & 10,000 & 6,300 & $63 \times 10^{6}$ \\
\hline $2062-1$ & 100 pounds & $1000 \mathrm{~s}$ tons & 20,000 & 7,250 & $145 \times 10^{6}$ \\
\hline $2063-1$ & $\begin{array}{l}\text { Pound of } \\
\text { input }\end{array}$ & $1000 \mathrm{~s}$ tons & $2,000,000$ & 23279 & $46,558 \times 10^{6}$ \\
\hline $2075-1$ & Pand of oil & Million pands & $1,000,000$ & 16,512 & $16,512 \times 10^{6}$ \\
\hline $2082-1$ & Pound & $1000 \mathrm{bbl}$ & $2.624 \times 10^{5}$ & & $40,962 \times 10^{6}$ \\
\hline $2221-1$ & Pand & $\begin{array}{l}\text { Mllition } 1 \text { inear } \\
\text { yards }\end{array}$ & $229 \times 10^{6}$ & 6,568 & $15,040 \times 10^{6}$ \\
\hline $2262-1$ & Pand & $\begin{array}{l}\text { Mllion finished } \\
\text { linear yard }\end{array}$ & $2.29 \times 10^{6}$ & 6,731 & $15,413 \times 10^{6}$ \\
\hline $2411-1$ & $\begin{array}{l}\text { Pound rough } \\
\text { ait }\end{array}$ & $\begin{array}{l}\text { Nillion feet } \\
(\log \text { seale) }\end{array}$ & $4.99 \times 10^{6}$ & 16,776 & $83,711 \times 10^{6}$ \\
\hline $2421-1$ & Pounds & $\begin{array}{l}\text { Million board } \\
\text { feet }\end{array}$ & $2 \times 10^{6}$ & 37,096 & $74,192 \times 10^{6}$ \\
\hline $2499-1$ & Square feet & $\begin{array}{l}\text { Million square } \\
\text { feet }\end{array}$ & $1,000,000$ & 506.3 & $506 \times 10^{6}$ \\
\hline $2611-1$ & Pound & $1000 \mathrm{~s}$ tons & $2,000,000$ & 4,717 & $9,435 \times 10^{6}$ \\
\hline $2611-2$ & Pound & $1000 \mathrm{~s}$ tans & $2,000,000$ & 726 & $1,452 \times 10^{6}$ \\
\hline $2611-3$ & Pound & $1000 \mathrm{~s}$ tons & $2,000,000$ & 122 & $244 \times 10^{6}$ \\
\hline $2621-1$ & Pound & $1000 \mathrm{~s}$ tans & $2,000,000$ & & $8,900 \times 10^{6}$ \\
\hline $2621-2$ & Pand & $1000 \mathrm{~s}$ tons & $2,000,000$ & 20,150 & $40,300 \times 10^{6}$ \\
\hline $2531-1$ & Pound & $1000 \mathrm{~s}$ tons & $2,000,000$ & & $9,400 \times 10^{6}$ \\
\hline
\end{tabular}




\section{TABLE 4.7 (contd)}

\begin{tabular}{|c|c|c|c|c|c|}
\hline $\begin{array}{l}\text { Drexel } \\
\text { Industry } \\
\text { Code } \\
\end{array}$ & $\begin{array}{l}\text { Drexel } \\
\text { Units of } \\
\text { Prodiction } \\
\end{array}$ & $\begin{array}{c}\text { Census of } \\
\text { Mnufacturers } \\
\text { Units of Production } \\
\end{array}$ & $\begin{array}{l}\text { Conversion from } \\
\text { Orexel Units to } \\
\text { Cand (nits } \\
\end{array}$ & $\begin{array}{c}1977 \\
\text { cyla) } \\
\text { Production } \\
\end{array}$ & $\begin{array}{l}\text { Mul tipl ler for } \\
\text { Converting Drexel } \\
\text { Data to Annual Data } \\
\end{array}$ \\
\hline $2631-2$ & Pand & $1000 \mathrm{~s}$ tons & $2,000,000$ & & $54,800 \times 10^{6}$ \\
\hline $2653-1$ & Square foot & $1000 \mathrm{~s}$ tons & $13,888.87 \times 10^{3}$ & $15,449.2$ & $214,572 \times 10^{6}$ \\
\hline 2661-1 & Pand & $1000 \mathrm{~s}$ tans & $2,000,000$ & & $3,600 \times 10^{6}$ \\
\hline $2661-2$ & Pand & $1000 \mathrm{~s}$ tons & $2, \infty 00,000$ & & $7,000 \times 10^{6}$ \\
\hline $2812-1$ & Pand & $1000 \mathrm{~s}$ tans & $2,000,000$ & & $17,000 \times 10^{6}$ \\
\hline $2812-2$ & Pand & $1000 \mathrm{~s}$ tans & $2,000,000$ & & $4,300 \times 10^{6}$ \\
\hline $2812-3$ & Pound & $1000 \mathrm{~s}$ tons & $2,000,000$ & & $8,100 \times 10^{6}$ \\
\hline $2812-4$ & Pound & Milition pounds & $2,000,000$ & & $8,000 \times 10^{6}$ \\
\hline $2813-1$ & Pound & Mllion $\mathrm{ft}^{3}$ & 89,200 & & $31,600 \times 10^{6}$ \\
\hline $2813-2$ & Pand & Million $\mathrm{ft}^{3}$ & 5,610 & & $445 \times 10^{6}$ \\
\hline $2813-3$ & Pound & Million $\mathrm{ft}^{3}$ & 84,160 & & $405 \times 10^{6}$ \\
\hline $2816-1$ & Pand & $1000 \mathrm{~s}$ tans & $2,000,000$ & & $750 \times 10^{6}$ \\
\hline $2816-2$ & Pand & $1000 \mathrm{~s}$ tons & $2,000,000$ & & $750 \times 10^{6}$ \\
\hline $2816-3$ & Pand & $1000 \mathrm{~s}$ tons & $2,000,000$ & & $130 \times 10^{6}$ \\
\hline $2816-4$ & Pand & $1000 \mathrm{~s}$ tans & $2,000,000$ & & $130 \times 10^{6}$ \\
\hline $2816-5$ & Pand & $1000 \mathrm{~s}$ tons & $2,000,000$ & & $130 \times 10^{6}$ \\
\hline 2819-1 & Pound & $1000 \mathrm{~s}$ tons & $2,000,000$ & & $11,900 \times 10^{6}$ \\
\hline $2819-2$ & Ton & $1000 \mathrm{~s}$ tons & 1,000 & & $36 \times 10^{6}$ \\
\hline $2819-3$ & Pand & $1000 \mathrm{~s}$ tans & $2,000,000$ & & $800 \times 10^{6}$ \\
\hline $2819-4$ & Pand & $1000 \mathrm{~s}$ tons & $2,000,000$ & & $4900 \times 10^{6}$ \\
\hline $2819-5$ & Pand & $1000 \mathrm{~s}$ tans & $2,000,000$ & & $320 \times 10^{6}$ \\
\hline $2821-1$ & Pand & Million pounds & $1,000,000$ & & $5,200 \times 10^{6}$ \\
\hline $2821-2$ & Pand & Mallion pounds & $1,000,000$ & & $5,400 \times 10^{6}$ \\
\hline $2821-3$ & Pand & Mfllion pounds & $1,000,000$ & & $6,500 \times 10^{6}$ \\
\hline $2821-4$ & Pound & Million pounds & $1,000,000$ & & $1,800 \times 10^{6}$ \\
\hline $2821-5$ & Pand & Mllion pounds & $1,000,000$ & & $1,800 \times 10^{6}$ \\
\hline $2822-1$ & Pand & Mllion pounds & $1,000,000$ & & $3,080 \times 10^{5}$ \\
\hline $2822-2$ & Pand & $1000 \mathrm{~s}$ tons & $2,000,000$ & & $330 \times 10^{6}$ \\
\hline $2822-3$ & Pand & Mllion pounds & $1,000,000$ & & $790 \times 10^{6}$ \\
\hline $2822-4$ & Pound & Mtlition pounds & $1, \infty 00,000$ & & $140 \times 10^{6}$ \\
\hline
\end{tabular}




\section{TABLE 4.7 (contd)}

\begin{tabular}{|c|c|c|c|c|c|}
\hline $\begin{array}{c}\text { Drexel } \\
\text { Industry } \\
\text { Code }\end{array}$ & $\begin{array}{c}\text { Drexel } \\
\text { Chits of } \\
\text { Production } \\
\end{array}$ & $\begin{array}{c}\text { Census of } \\
\text { Menufacturers } \\
\text { Units of Production } \\
\end{array}$ & $\begin{array}{l}\text { Conversion fram } \\
\text { Drexel (hits to } \\
\text { units } \\
\end{array}$ & $\begin{array}{c}1977 \\
\text { CM(a) } \\
\text { Production }\end{array}$ & $\begin{array}{l}\text { Multipl ier for } \\
\text { Converting Drexel } \\
\text { Data to Annual Data } \\
\end{array}$ \\
\hline $2822-5$ & Pand & Million pounds & $1,000,000$ & & $158 \times 10^{6}$ \\
\hline $2823-1$ & Pand & Million pounds & $1,000,000$ & & $606 \times 10^{6}$ \\
\hline $2823-2$ & Pound & Million pounds & $1,000,000$ & & $324 \times 10^{6}$ \\
\hline $2824-1$ & Pand & Million pands & $1,000,000$ & & $4,200 \times 10^{6}$ \\
\hline $2824-2$ & Pand & Mllion pounds & $1,000,000$ & 38000 & $3,800 \times 10^{6}$ \\
\hline $2824-3$ & Pand & Mllion pounds & $1,000,000$ & & $761 \times 10^{6}$ \\
\hline $2824-4$ & Pound & illion pounds & $1,000,000$ & 1900 & $1,900 \times 10^{6}$ \\
\hline $2824-5$ & Pound & Million pounds & $1,000,000$ & 600 & $600 \times 10^{6}$ \\
\hline $2834-1$ & \$ sales & Million \$ shiprents & 10 & 9,639 & $9,640 \times 10^{6}$ \\
\hline $2865-1$ & Pand & Billion pands & $10^{9}$ & & $6,900 \times 10^{6}$ \\
\hline $2865-2$ & Pand & Btllion pounds & $10^{9}$ & & $2,600 \times 10^{6}$ \\
\hline $2865-3$ & Pound & Billion pands & $10^{9}$ & & $2,300 \times 10^{6}$ \\
\hline $2869-1$ & Pand & Billion pounds & $10^{9}$ & & $25,200 \times 10^{6}$ \\
\hline $2869-2$ & Pand & Btllion pounds & $10^{9}$ & & $6,500 \times 10^{6}$ \\
\hline $2869-3$ & Pand & Btllion pounds & $10^{9}$ & & $6,000 \times 10^{6}$ \\
\hline $2873-1$ & Pand & $1000 \mathrm{~s}$ tans & $2,000,000$ & & $38,800 \times 10^{6}$ \\
\hline $2874-1$ & Pound & $1000 \mathrm{~s}$ tons & $2,000,000$ & & $15,200 \times 10^{6}$ \\
\hline $2874-2$ & Pand & $1000 \mathrm{~s}$ tons & $1,000,000$ & & $1,700 \times 10^{6}$ \\
\hline $2874-3$ & Pand & $1000 \mathrm{~s}$ tons & $2,000,000$ & & $4,000 \times 10^{6}$ \\
\hline $2874-4$ & Tons & 1000 s tons & 1,000 & & $9,600 \times 10^{6}$ \\
\hline $2899-1$ & Pand & Mllion s tons & $2 \times 10^{9}$ & & $1,030 \times 10^{6}$ \\
\hline $2899-2$ & Pound & itillion s tons & $2 \times 10^{9}$ & & $1,030 \times 10^{5}$ \\
\hline $2911-1$ & $\begin{array}{l}\text { Pand of } \\
\text { crude oil }\end{array}$ & $\begin{array}{l}\text { million BQR } \\
\text { crude sil }\end{array}$ & $308 \times 10^{6}$ & $5,108,5$ & $1,578 \times 10^{9}$ \\
\hline $2951-1$ & Pand & $1000 \mathrm{~s}$ tans & $2,000,000$ & 58,013 & $116,027 \times 10^{6}$ \\
\hline $301-1$ & Pand & Million pounds & $1,000,000$ & 8,946 & $8,946 \times 10^{6}$ \\
\hline $3069-1$ & Pand & Million pounds & $1,000,000$ & & $505 \times 10^{6}$ \\
\hline $3059-2$ & Pand & Million pounds & $1,000,000$ & & $506 \times 10^{5}$ \\
\hline $3069-3$ & Pand & Million pands & $1,000,000$ & & $1,990 \times 10^{6}$ \\
\hline $3079-1$ & Pand & Mllion pounds & $1,000,000$ & 16,633 & $16,533 \times 10^{6}$ \\
\hline
\end{tabular}




\section{TABLE 4.7. (contd)}

\begin{tabular}{|c|c|c|c|c|c|}
\hline $\begin{array}{l}\text { Drexel } \\
\text { Industry } \\
\text { Code }\end{array}$ & $\begin{array}{l}\text { Drexel } \\
\text { Units of } \\
\text { Production } \\
\end{array}$ & $\begin{array}{c}\text { Census of } \\
\text { Menufacturers } \\
\text { Units of Production } \\
\end{array}$ & $\begin{array}{l}\text { Conversion from } \\
\text { Drexel (nits to } \\
\text { ond inits } \\
\end{array}$ & $\begin{array}{c}1977 \\
\text { Cra (a) } \\
\text { Production }\end{array}$ & $\begin{array}{l}\text { Multiplier for } \\
\text { Converting Drexel } \\
\text { Data to Annual Data }\end{array}$ \\
\hline $3211-1$ & Pound & $1000 \mathrm{~s}$ tans & $2,000,000$ & 2,900 & $5,800 \times 10^{6}$ \\
\hline $3221-1$ & Pand & $1000 \mathrm{~s}$ tons & $2,000,000$ & 15,120 & $30,240 \times 10^{6}$ \\
\hline $3220-1$ & Pound & $1000 \mathrm{~s}$ tons & $2,000,000$ & 1635 & $3270 \times 10^{6}$ \\
\hline $3241-1$ & Pand & Mllion s tons & $2 \times 10^{9}$ & 77.9 & $155,800 \times 10^{6}$ \\
\hline $3251-1$ & Pound & $1000 \mathrm{~s}$ tans & $2,000,000$ & 9895 & $19,790 \times 10^{6}$ \\
\hline $3273-1$ & Pand & Million yards ${ }^{3}$ & $2.54 \times 10^{9}$ & 203.4 & $516,600 \times 10^{6}$ \\
\hline $3274-1$ & Pound & $1000 \mathrm{~s}$ tons & $2,000,000$ & 1395.3 & $2,790 \times 10^{6}$ \\
\hline $3275-1$ & Pand & $1000 \mathrm{~s}$ tons & $2,000,000$ & 13,300 & $26,600 \times 10^{6}$ \\
\hline $3296-1$ & Pound & $1000 \mathrm{~s}$ tons & $2,000,000$ & 1630 & $3260 \times 10^{6}$ \\
\hline $3312-1$ & Pand & $1000 \mathrm{~s}$ tons & $2,000,000$ & & $200,000 \times 10^{6}$ \\
\hline $3313-1$ & Pound & $1000 \mathrm{~s}$ tans & $2,000,000$ & & $4,360 \times 10^{6}$ \\
\hline $3321-1$ & Pand & $1000 \mathrm{~s}$ tons & $2,000,000$ & & $25,000 \times 10^{6}$ \\
\hline $3331-1$ & Pound & $1000 \mathrm{~s}$ tans & $2,000,000$ & & $7,160 \times 10^{6}$ \\
\hline $3334-1$ & Pound & $1000 \mathrm{~s}$ tons & $2,000,000$ & & $9,280 \times 10^{6}$ \\
\hline $3341-1$ & Pound & $1000 \mathrm{~s}$ tans & $2,000,000$ & & $2,300 \times 10^{6}$ \\
\hline $3341-2$ & Pand & $1000 \mathrm{~s}$ tons & $2,000,000$ & & $1570 \times 10^{6}$ \\
\hline $3341-3$ & Pound & $1000 \mathrm{~s}$ tons & $2,000,000$ & & $442 \times 10^{6}$ \\
\hline $3341-4$ & Pand & $1000 \mathrm{~s}$ tons & $2,000,000$ & & $918 \times 10^{6}$ \\
\hline $3353-1$ & Pand & $1000 \mathrm{~s}$ tons & $2,000,000$ & & $8,240 \times 10^{6}$ \\
\hline $3462-1$ & Pound & $1000 \mathrm{~s}$ tans & $2,000,000$ & & $2,500 \times 10^{6}$ \\
\hline $3523-1$ & \# sales & Mllion \$ shiptents & $1,000,000$ & $\$ 9548.9$ & $9,550 \times 10^{6}$ \\
\hline $3531-1$ & $\$$ sales & Milion \$ shipments & $1,000,000$ & $\$ 11,569.9$ & $11,570 \times 10^{6}$ \\
\hline $3711-1$ & Pounds & Mallion pounds & $1,000,000$ & & $16,600 \times 10^{6}$ \\
\hline $374-1$ & Pounds & Million pands & $1,000,000$ & & $32,100 \times 10^{6}$ \\
\hline $3861-1$ & Pounds & Mllion pounds & $1,000,000$ & & $1,200 \times 10^{6}$ \\
\hline $3851-2$ & $\$$ of sales & Million $\$$ sales & $1,000,000$ & $\$ 1104$ & $1,104 \times 10^{6}$ \\
\hline
\end{tabular}


TABLE 4.8 Comparison of Industrial Production Estimates (G.E. 1981)

\begin{tabular}{|c|c|c|c|c|c|}
\hline $\begin{array}{l}\text { Drexel } \\
\text { Code } \\
\end{array}$ & $\begin{array}{l}\text { SIC } \\
\text { Code } \\
\end{array}$ & $\begin{array}{l}\text { Units In } \\
\text { Millions }\end{array}$ & $\frac{\text { U.S. Produe }}{\text { Value Used }}$ & $\frac{n \text { Estimate }}{\mathrm{GE}^{(1)}}$ & $\begin{array}{c}\text { Value } \\
\text { Used/GE } \\
\end{array}$ \\
\hline $2011-1$ & 2011 & Pounds & 31,675 & 25,000 & 1.27 \\
\hline $2026-1$ & 2026 & Pounds & 61,768 & 52,000 & 1.19 \\
\hline $2033-1$ & 2033 & Cases & 151 & 230 & 0.66 \\
\hline $2033-2$ & 2033 & Cases & 251 & 295 & 0.85 \\
\hline $2033-3$ & 2033 & Cases & 157 & 220 & 0.71 \\
\hline $2046-1$ & 2046 & Pounds & 15,180 & 22,000 & 0.69 \\
\hline 2051-1 & 2051 & Hundred wt. & 109 & 109 & 1.00 \\
\hline $2051-2$ & 2051 & Hundred wt. & 63 & 67 & 0.94 \\
\hline 2062-1 & 2062 & Hundred Wt. & 145 & 145 & 1.00 \\
\hline $2063-1$ & 2063 & Pounds & 46,558 & 11,110 & 4.19 \\
\hline $2075-1$ & 2075 & Pounds & 16,512 & 9,500 & 1.74 \\
\hline $2082-1$ & 2082 & Pounds & 40,962 & 38,000 & 1.08 \\
\hline $2221-1$ & 2221 & Pounds & 15,040 & 1,900 & 7.92 \\
\hline $2262-1$ & 2262 & Pounds & 15,413 & 1,000 & 15.4 \\
\hline $2411-1$ & 2411 & Pounds & 83,711 & 101 Cords & - \\
\hline $2421-1$ & 2421 & Pounds & 74,192 & 41,000 & 1.81 \\
\hline $2499-1$ & 2499 & sq. ft. & 506 & 594 & 0.85 \\
\hline $2611-1$ & 2611 & Pounds & 9,435 & 5,400 & 1.89 \\
\hline $2611-2$ & 2611 & Pounds & 1,452 & 1,100 & 1.32 \\
\hline $2611-3$ & 2611 & Pounds & 244 & 700 & 0.35 \\
\hline $2621-1$ & 2621 & Pounds & 8,900 & 8,900 & 1.00 \\
\hline $2621-2$ & 2621 & Pounds & 40,300 & 40,300 & 1.00 \\
\hline $2631-1$ & 2631 & Pounds & 9,400 & 9,400 & 1.00 \\
\hline $2631-2$ & 2631 & Pounds & 54,800 & 54,800 & 1.00 \\
\hline $2653-1$ & 2653 & sq. ft. & 214,572 & 260,600 & 0.83 \\
\hline $2661-1$ & 2661 & Pounds & 3,600 & 3,600 & 1.00 \\
\hline $2661-2$ & 2661 & Pounds & 7,000 & 7,000 & 1.00 \\
\hline $2812-1$ & 2812 & Pounds & 17,000 & 17,000 & 1.00 \\
\hline $2812-2$ & 2812 & Pounds & 4,300 & 4,300 & 1.00 \\
\hline $2812-3$ & 2812 & Pounds & 8,100 & 8,100 & 1.00 \\
\hline $2812-4$ & 2812 & Pounds & 8,000 & 8,000 & 1.00 \\
\hline $2813-1$ & 2813 & Pounds & 31,600 & 31,600 & 1.00 \\
\hline $2813-2$ & 2813 & Pounds & 445 & 445 & 1.00 \\
\hline $2813-3$ & 2813 & Pounds & 405 & 405 & 1.00 \\
\hline $2816-1$ & 2816 & Pounds & 750 & 750 & 1.00 \\
\hline $2816-2$ & 2815 & Pounds & 750 & 750 & 1.00 \\
\hline $2816-3$ & 2816 & Pounds & 130 & 130 & 1.00 \\
\hline
\end{tabular}

(1) Industrial Process Heat Pump Service Study prepared by General ETectric Company for U.S. Department of Energy April 1981. 
TABLE 4.8 (contd)

\begin{tabular}{|c|c|c|c|c|c|}
\hline $\begin{array}{l}\text { Drexe1 } \\
\text { Code } \\
\end{array}$ & $\begin{array}{l}\text { SIC } \\
\text { Code }\end{array}$ & $\begin{array}{l}\text { Units In } \\
\text { Militions }\end{array}$ & $\frac{\text { U.S. Product }}{\text { Value Used }}$ & $\frac{\text { Estimate }}{G E(1)}$ & $\begin{array}{r}\text { Value } \\
\text { Used/GE }\end{array}$ \\
\hline $2816-4$ & 2816 & Pounds & 130 & 130 & 1.00 \\
\hline $2816-5$ & 2816 & Pounds & 130 & 130 & 1.00 \\
\hline $2819-1$ & 2819 & Pounds & 11,900 & 11,900 & 1.00 \\
\hline $2819-2$ & 2819 & Tons & 36 & 36 & 1.00 \\
\hline $2819-3$ & 2819 & Pounds & 800 & 800 & 1.00 \\
\hline $2819-4$ & 2819 & Pounds & 4,900 & 4,900 & 1.00 \\
\hline $2819-5$ & 2819 & Pounds & 320 & 320 & 1.00 \\
\hline $2821-1$ & 2821 & Pour.ds & 5,200 & 5,200 & 1.00 \\
\hline $2821-2$ & 2821 & Pounds & 5,400 & 5,400 & 1.00 \\
\hline $2821-3$ & 2821 & Pounds & 6,500 & 6,500 & 1.00 \\
\hline $2821-4$ & 2821 & Pounds & 1,800 & 1,800 & 1.00 \\
\hline $2821-5$ & 2821 & Pounds & 1,800 & 1,800 & 1.00 \\
\hline $2822-1$ & 2822 & Pounds & 3080 & 3080 & 1.00 \\
\hline $2822-2$ & 2822 & Pounds & 330 & 330 & 1.00 \\
\hline $2822-3$ & 2822 & Pounds & 790 & 790 & 1.00 \\
\hline $2822-4$ & 2822 & Pounds & 140 & 140 & 1.00 \\
\hline $2822-5$ & 2822 & Pounds & 158 & 158 & 1.00 \\
\hline $2823-1$ & 2823 & Pounds & 606 & 606 & 1.00 \\
\hline $2823-2$ & 2823 & Pounds & 324 & 324 & 1.00 \\
\hline $2824-1$ & 2824 & Pounds & 4,200 & 4,200 & 1.00 \\
\hline $2824-2$ & 2824 & Pounds & 3,800 & 3,800 & 1.00 \\
\hline $2824-3$ & 2824 & Pounds & 761 & 761 & 1.00 \\
\hline $2824-4$ & 2824 & Pounds & 1,900 & 1,900 & 1.00 \\
\hline $2824-5$ & 2824 & Pounds & 600 & 600 & 1.00 \\
\hline $2834-1$ & 2834 & \$ sales & 9,640 & 79 Btu E12 & - \\
\hline $2865-1$ & 2865 & Pounds & 6,900 & 6,900 & 1.00 \\
\hline $2865-2$ & 2865 & Pounds & 2,600 & 2,600 & 1.00 \\
\hline $2865-3$ & 2865 & Pounds & 2,300 & 2,300 & 1.00 \\
\hline $2869-1$ & 2869 & Pounds & 25,200 & 25,200 & 1.00 \\
\hline $2869-2$ & 2869 & Pounds & 6,500 & 6,500 & 1.00 \\
\hline $2869-3$ & 2869 & Pounds & 6,000 & 6,000 & 1.00 \\
\hline $2873-1$ & 2873 & Pounds & 38,800 & 38,800 & 1.00 \\
\hline $2874-1$ & 2874 & Pounds & 15,200 & 15,200 & 1.00 \\
\hline $2874-2$ & 2874 & Pounds & 1,700 & 1,700 & 1.00 \\
\hline $2874-3$ & 2874 & Pounds & 4,000 & 4,000 & 1.00 \\
\hline $2874-4$ & 2874 & Pounds & 9,600 & 9,600 & 1.00 \\
\hline $2899-1$ & 2899 & Pounds & 1,030 & 1,030 & 1.00 \\
\hline
\end{tabular}


TABLE 4.8 (contd)

\begin{tabular}{|c|c|c|c|c|c|}
\hline $\begin{array}{l}\text { Drexel } \\
\text { Code }\end{array}$ & $\begin{array}{l}\text { SIC } \\
\text { Code }\end{array}$ & $\begin{array}{l}\text { Units In } \\
\text { Millions }\end{array}$ & $\frac{\text { U.S. Produc }}{\text { Value Used }}$ & $\frac{\text { ion Estimate }}{G^{(1)}}$ & $\begin{array}{c}\text { Value } \\
\text { Used/GE }\end{array}$ \\
\hline $2899-2$ & 2899 & Pounds & 1,030 & 1,030 & 1.00 \\
\hline $2911-1$ & 2911 & Pounds & $1,578,000$ & $1,46,000$ & 1.00 \\
\hline $2951-1$ & 2951 & Pounds & 116,027 & 9,300 & 12.5 \\
\hline $3011-1$ & 3011 & Pounds & 8,946 & 10,200 & 0.88 \\
\hline $3069-1$ & 3069 & Pounds & 505 & 505 & 1.00 \\
\hline $3069-2$ & 3069 & Pounds & 506 & 506 & 1.00 \\
\hline $3069-3$ & 3069 & Pounds & 1990 & 1,990 & 1.00 \\
\hline $3079-1$ & 3079 & Pounds & 16,633 & 15,800 & 1.05 \\
\hline $3211-1$ & 3211 & Pounds & 5,800 & 12,700 & 0.69 \\
\hline $3221-1$ & 3221 & Pounds & 30,240 & 23,400 & 1.29 \\
\hline $3229-1$ & 3229 & Pounds & 3,270 & 2,440 & 1.34 \\
\hline $3241-1$ & 3241 & Pounds & 155,800 & 168,000 & 0.93 \\
\hline $3251-1$ & 3251 & Pounds & 19,790 & 54,400 & 0.36 \\
\hline $3273-1$ & 3273 & Pounds & 516,600 & 34,400 & 15.0 \\
\hline $3274-1$ & 3274 & Pounds & 2,790 & 29,200 & 0.10 \\
\hline $3275-1$ & 3275 & Pounds & 26,600 & 33,600 & 0.79 \\
\hline $3296-1$ & 3296 & Pounds & 3,260 & 3,660 & 0.89 \\
\hline $3312-1$ & 3312 & Pounds & 290,000 & 290,000 & 1.00 \\
\hline $3313-1$ & 3313 & Pounds & 4,360 & 4,360 & 1.00 \\
\hline $3321-1$ & 3321 & Pounds & 25,000 & 25,000 & 1.00 \\
\hline $3331-1$ & 3331 & Pounds & 7,160 & 7,160 & 1.00 \\
\hline $3334-1$ & 3334 & Pounds & 9,280 & 9,280 & 1.00 \\
\hline $3341-1$ & 3341 & Pounds & 2,300 & 2,300 & 1.00 \\
\hline $3341-2$ & 3341 & Pounds & 1570 & 1,570 & 1.00 \\
\hline $3341-3$ & 3341 & Pounds & 442 & 442 & 1.00 \\
\hline $3341-4$ & 3341 & Pounds & 918 & 918 & 1.00 \\
\hline $3353-1$ & 3353 & Pounds & 8,240 & 8,240 & 1.00 \\
\hline $3462-1$ & 3462 & Pounds & 2,500 & 2,500 & 1.00 \\
\hline $3523-1$ & 3523 & $\$$ sales & 9,550 & $5,800 \mathrm{lbs}$ & -- \\
\hline $3531-1$ & 3531 & $\$$ sales & 11,570 & 13 units & -- \\
\hline $3711-1$ & 3711 & Pounds & 16,600 & 16,600 & 1.00 \\
\hline $3714-1$ & 3714 & Pounds & 32,100 & 32,100 & 1.00 \\
\hline $3861-1$ & 3861 & Pounds & 1,200 & 1,200 & 1.00 \\
\hline $3861-2$ & 3861 & $\$$ sales & 1,104 & 18 units & -- \\
\hline
\end{tabular}


Adjustment Factors

Review of the waste heat stream energy content data, calculated by multiplying Drexel process data by the production multipliers, indicated that for 12 specific processes the calculated energy content appears to be unreasonably high. Therefore, specific adjustment factors for each of these processes were developed. The adjustment factor and the basis for its value is shown in Table 4.9. For processes 2411-1 and 2874-4, adjustments are necessary to correct the production unit discrepancies between those initially indicated by the Drexel data base and the one actually used. The other processes required

\section{TABLE 4.9 Adjustment Factors}

$\begin{array}{lll}\begin{array}{c}\text { Process } \\ \text { Number }\end{array} & \begin{array}{c}\text { Adjustment } \\ \text { Factor }\end{array} & \end{array}$


adjustments to more closely relate expected waste heat stream energy content with industry input energy as specified by the 1980 Survey of Manufactures information on purchased fuels. For some industries, the comparison of purchased fuels to waste heat is not a good one because the industry may generate energy from feedstock materials. However, for these 10 industries the developed adjustment factors appear reasonable.

\section{WASTE HEAT STREAM ENERGY CONTENT}

The industry energy flow data base as discussed above estimates the annual energy content in specific waste heat streams (gas, water, and steam) and specific input heat streams (process air, water, and steam).

Waste heat stream and energy input stream information was compiled for a11 108 industrial processes. Each industrial process contains an average of 15 unit operations and each unit operation often contains more than one energy stream. Table 4.10 illustrates the type of information available from the industry energy flow data base. The header for Table 4.10 includes process number and name. The first four digits of the code relate to the SIC code (for example, 2011 - Meat Packing Plant) and the fifth digit reflects the number of the process addressed. The table header also gives the number of plants classified under the five-digit process code $(2580)$, the expected annual hours of operation (2100), and the annual production (31.7 billion pounds). The table contains the following information:

- process flow, input or waste

0 type of heat stream, e.g., gas, liquid

0 the temperature of the stream. Temperatures are arranged from lowest to highest within each stream type.

0 energy content of the stream in Btu per unit of product. (The unit of product is given in the header information.)

o total energy content of each stream in trillions of Btu annually. This number is calculated by multiplying the energy content per unit of product by the annual production rate. (Annual production information is also given in the table header.) 


\section{TABLE 4.10 Steam Flow Energy Content Data}

\begin{tabular}{|c|c|c|c|c|c|}
\hline $\begin{array}{l}\quad \text { Process } \\
\text { Energy Flows } \\
\text { Input/Waste } \\
\end{array}$ & Stream & Temp (F) & \multicolumn{2}{|c|}{$\begin{array}{l}\text { Energy Content } \\
\text { (Btu/Unit } \\
\text { Production) } \\
\end{array}$} & $\begin{array}{r}\text { Total Energy } \\
\left(10{ }^{12} \mathrm{Btu} / \mathrm{yr}\right) \\
\end{array}$ \\
\hline \multirow[t]{2}{*}{ Input } & Gas & 250 & $\begin{array}{r}73.0 \\
83.4 \\
127.8 \\
511.0 \\
73.0 \\
31.0\end{array}$ & Total & $\begin{array}{r}2.31 \\
2.64 \\
4.05 \\
16.19 \\
2.31 \\
0.99 \\
28.49\end{array}$ \\
\hline & Liquid & 140 & $\begin{array}{r}85.2 \\
170.3\end{array}$ & Total & $\begin{array}{l}2.70 \\
5.39 \\
8.09\end{array}$ \\
\hline \multirow[t]{6}{*}{ Waste } & Gas & 212 & $\begin{array}{l}19.5 \\
82.4\end{array}$ & Total & $\begin{array}{l}0.62 \\
2.61 \\
3.23\end{array}$ \\
\hline & & 220 & 57.7 & Total & $\frac{1.83}{1.83}$ \\
\hline & & 400 & 123.2 & Total & $\frac{3.90}{3.90}$ \\
\hline & & 500 & $\begin{array}{r}38.4 \\
590.5\end{array}$ & Total & $\begin{array}{r}1.22 \\
\frac{18.70}{19.92}\end{array}$ \\
\hline & Liquid & 95 & 420.5 & Total & $\frac{13.32}{13.32}$ \\
\hline & & 120 & $\begin{array}{r}69.5 \\
138.2\end{array}$ & Total & $\begin{array}{l}2.20 \\
4.38 \\
6.58\end{array}$ \\
\hline
\end{tabular}

SIC number is: $2011-1$

Industry: meat packing plants

Number of plants: 2580

Annual hours: 2100

Annual production: 31.7 billion

Units of production: pounds

\begin{tabular}{l} 
Process \\
Energy Flows \\
Input/Waste \\
\hline
\end{tabular}

Input

Waste
Gas

- the total energy in the input/output stream(s) for each temperature. Within a particular process, several energy input streams may have the same temperature value. For example, a stream at $250^{\circ} \mathrm{F}$ may be used in several unit operations. Similarly, several unit operations may exhaust gas at, for example, $500^{\circ} \mathrm{F}$.

Waste Heat by Industry

The industry energy flow data base was used to estimate the energy content for each waste heat stream within an industrial process and to total these 
values for all gaseous and liquid waste streams with temperatures above $80^{\circ} \mathrm{F}$. Table 4.11 estimates the annual waste heat rejected by each of the 108 industrial processes. The total energy content of waste heat streams for all 108 industrial processes is estimated to be 6,670.1 trillion Btu (6.7 quad). The five processes with the highest waste heat stream energy content are Petroleum Refining $(1,740.7)$, Blast Furnaces (898.4), Integrated Paper Board Mills (575.6), Integrated Paper Mills (490.8), and Cement (251.3)(units are $\left.10^{12} \mathrm{Btu}\right)$.

Waste Heat by Type of Equipment

The terms used in describing various unit operations within the 108 industrial processes are not narrowly defined. For example, Furnaces are classified to include the following unit operations: Autoclave, Blast Furnace, Burner, Distillation Furnace, Electric Furnace, Furnace, Induction Furnace, Kettle Furnace, Kiln, Melting Furnace, Open Hearth Furnace, Recovery Furnace, Reforming Furnace, Reheat Furnace, Rotary Furnace, Smelting Furnace, Sweating Furnace and 0thers. Given that the description of the unit operation and its related equipment is broadly defined, Table 4.12 estimates the level of waste heat associated with each generic type of equipment. The five generic types of equipment having the largest waste heat energy streams are Furnaces $(1,217.4)$, Distillation Equipment (939.7), Drying Equipment (778.4), Boilers (575.2), and Thermal-Chemical Reactors (486.2)(units are $10^{12}$ Btu). 
TABLE 4.11. Estimate of Waste Heat by Industry

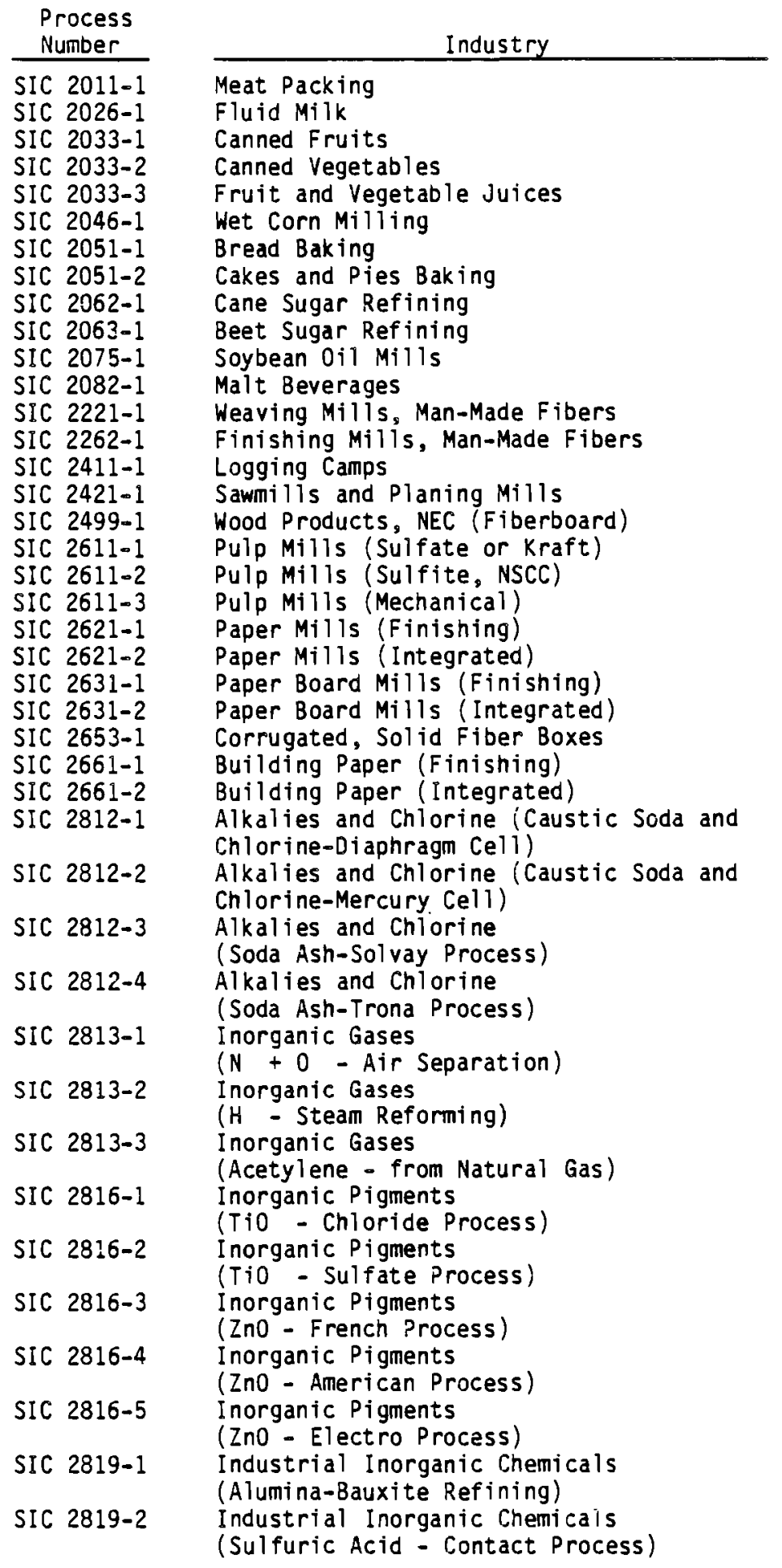

Trillion

Btu

48.8

21.4

4.7

8.1

3.6

37.2

4.7

2.4

29.2

53.2

36.2

41.9

31.6

23.5

0.3

144.7

4.1

59.0

5.3

0.9

42.3

490.8

49.8

575.6

15.4

14.4

30.0

49.8

6.7

5.8

7.8

19.8

36.2

19.0

2.6

14.7

1.3

3.9

1.6

84.4

2.3 
TABLE 4.11. (contd)

Process

Number

SIC 2819-3

SIC 2819-4

SIC 2819-5

SIC 2821-1

SIC 2821-2

SIC 2821-3

SIC 2821-4

SIC 2821-5

SIC 2822-1

SIC 2822-2

SIC 2822-3

SIC 2822-4

SIC 2822-5

SIC 2823-1

SIC 2823-2

SIC 2824-1

SIC 2824-2

SIC 2824-3

SIC 2824-4

SIC 2824-5

SIC 2334-1

SIC 2865-1

SIC 2865-2

SIC 2865-3

SIC 2869-1

SIC 2869-2

SIC 2869-3

SIC 2873-1

SIC 2874-1

SIC 2874-2

SIC 2874-3

SIC 2874-4
Industry

Industrial Inorganic Chemicals (Phosphorous-Elect Furnace)

Industrial Inorganic Chemicals (Potash-Fiotation Method)

Industrial Inorganic Chemicals

(Sodium Metal - Electrolysis)

Plastic Materials and Resins

(PVC) Suspension

Plastic Materials and Resins

(Polystyrene) Mass

Plastic Materials and Resins

(LDPE)

Plastic Materials and Resins

(HDPE) Slurry Process

Plastic Materials and Resins

(HDPE) Solution Process

Synthetic Rubbers (SBR)

Synthetic Rubbers (Buty 1)

Synthet ic Rubbers (Polybutadiene)

Synthetic Rubbers (Polyisoprene)

Synthetic Rubbers (EP Rubber)

Cellulosic Man-Made Fibers (Rayon)

Cellulosic Man-Made Fibers (Acetate)

Organic Fibers, Non-Cellulosic (Polyester)

Organic Fibers, Non-Cellulosic

(Polypropylene)

Organic Fibers, Non-Cellulosic

(Acrylics)

Organic Fibers, Non-Celluiosic

(Nylon, 6,6)

Organic Fibers, Non-Celluiosic (Nylon 6)

Pharmaceutical Preparations

Cyclic Crudes and Intermediates

(Ethylbenzene and Styrene)

Cyclic Crudes and Intermediates

(Cumene and Phenol)

Cyclic Crudes and Intermediates

(Cyclohexane)

Industrial Organic Chemicals

(Ethylene)

Industrial Organic Chemicals

(Methanol)

Industrial Organic Chemicals

(Forma ldehyde)

Nitrogenous Fertilizers

(Armonia-Gas Reforming)

Phosphatic Fertilizers

(Phorphoric Acid-Wet Digestion)

Phosphatic Fertilizers

(Phosphoric Acid-Furnace)

Phosphatic Fertilizers

(TSP-Superphosphates)

Phosphatic Fertilizers

(Ammonium-Phosphates)
Trillion

Btu

6.9

5.6

1.1

5.8

3.2

26.2

3.4

3.9

8.8

5.3

11.3

1.7

1.8

20.5

4.9

56.7

48.4

21.7

17.6

4.04

6.1

97.2

28.1

6.1

198.3

33.4

7.4

247.2

28.3

28.6

3.0

2.8 
TABLE 4.11. (contd)

Process
Number

SIC 2899-1

SIC 2899-2

SIC 2911-1

SIC 2951-1

SIC 3011-1

SIC 3069-1

SIC 3069-2

SIC 3069-3

SIC 3079-1

SIC 3211-1

SIC 3221-1

SIC 3229-1

SIC 3241-1

SIC 3251-1

SIC 3273-1

SIC 3274-1

SIC 3275-1

SIC 3296-1

SIC 3312-1

SIC 3313-1

SIC 3321-1

SIC 3331-1

SIC 3334-1

SIC 3341-1

SIC $3341-2$

SIC $3341-3$

SIC 3341-4

SIC 3353-1

SIC 3462-1

SIC 3523-1

SIC 3531-1

SIC 3711-1

SIC 3714-1

SIC 3861-1

SIC 3861-2
Industry

Chemical Preparations, NEC (Salts)

Chemical Preparations (Fatty Acids)

Petroleum Refining

Paving Mixtures and Blocks

Tires and Inner Tubes

Fabricated Rubber Products, NEC

(Dipped Latex)

Fabricated Rubber Products, NEC

(Molded Latex)

Fabricated Rubber Products, NEC

(Molded Rubber)

Miscellaneous Plastic Products

Flat Glass

Glass Containers

Pressed and Blown Glass

Cement, Hydraulic

Brick and Structural Clay Tile

Ready Mix Cement

Lime

Gypsum Products

Mineral Wood

Blast Furnaces and Steel Mills

Electrometallurgical Products

Gray Iron Foundries

Primary Copper

Primary Alumi num

Secondary Non-Ferrous Metals

(Alumi num)

Secondary Non-Ferrous Metals

(Lead)

Secondary Non-Ferrous Metals

(Zinc)

Secondary Non-Ferrous Metals

(Copper)

Aluminum Finish Forming

Iron and Steel Forging

Farm Machinery and Equipment

Construction Machinery

Motor Vehicles and Car Bodies

Motor Vehicles Parts and Accessories

Photographic Film

Photographic Equipment -

Fabrication and Assembly

Total for all 108 processes

(Total may differ from sum of process values due to rounding.)
Trillion

Btu

1.4

1.7

$1,740.7$

7.2

9.3

9.7

9.6

10.7

35.6

12.9

89.2

11.0

251.3

32.9

1.2

3.8

27.3

18.8

898.4

16.5

117.7

46.5

89.6

3.7

0.5

0.7

1.0

21.8

32.8

30.7

32.3

44.4

74.2

0.5

3.5

$6,670.1$ 
TABLE 4.12. Estimate of Waste Heat by Type of Process Equipment

\begin{tabular}{|c|c|}
\hline Type of Equipment & $\begin{array}{c}\text { Trilition } \\
\text { Btu } \\
\end{array}$ \\
\hline $\begin{array}{l}\text { Absorption } \\
\text { Annealing } \\
\text { Boiler } \\
\text { Casting, molding } \\
\text { Centrifuge } \\
\text { Coking } \\
\text { Compressor } \\
\text { Condenser } \\
\text { Cooking } \\
\text { Cooling } \\
\text { Crystallization } \\
\text { Crushing, grinding } \\
\text { Cutting, trimming } \\
\text { Distillation } \\
\text { Orying } \\
\text { Electrical generation } \\
\text { Electrolytic cells } \\
\text { Extraction } \\
\text { Extruding } \\
\text { Flash separator } \\
\text { Filter } \\
\text { Furnace } \\
\text { Heat exchanger } \\
\text { Heating } \\
\text { Leaching } \\
\text { Mechanical prime mover } \\
\text { Mechanical processing } \\
\text { Mixing } \\
\text { Ovens } \\
\text { Packaging } \\
\text { Pasturizing } \\
\text { Rolling } \\
\text { Separation } \\
\text { Settling } \\
\text { Storage, holding tank } \\
\text { Thermal-chemical reactors } \\
\text { Washing } \\
\text { Other equipment } \\
\text { Total }\end{array}$ & $\begin{array}{r}70.3 \\
93.3 \\
575.2 \\
51.8 \\
1.1 \\
34.4 \\
82.4 \\
53.0 \\
18.2 \\
202.2 \\
5.9 \\
16.0 \\
1.0 \\
939.7 \\
778.4 \\
358.1 \\
27.0 \\
12.7 \\
0.8 \\
1.4 \\
9.1 \\
1,217.4 \\
85.3 \\
93.1 \\
13.5 \\
83.8 \\
0.8 \\
7.8 \\
259.5 \\
2.4 \\
8.3 \\
157.8 \\
68.4 \\
3.0 \\
126.9 \\
486.2 \\
26.6 \\
696.3 \\
6,670.1\end{array}$ \\
\hline
\end{tabular}




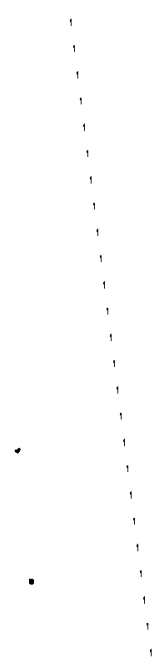




\subsection{SUBSTITUTING WASTE HEAT FOR PROCESS HEAT NEEDS}

The usefulness (utility) of any energy recovered from waste heat streams depends largely on the immediate energy input needs of the plant or process from which the heat emanates. However, the energy supplied by waste heat recovery technology does not need to match the time of need exactly. Using existing or advanced technology, the energy can be stored.

Recovered waste heat can displace part or all of the energy input needs for specific unit operations within an industrial plant. A broad spectrum of advanced energy recovery technologies exist that could be used in recovering energy from waste heat streams. The various waste heat recovery equipment available, or soon to be available, is discussed in the Task 2 report for this project. Government and industry also are studying advanced waste heat recovery materials, components and systems that could be available in the future.

This analysis of waste heat streams and the recoverable energy is generic in that it is not tied to any particular recovery technology. Looking across industries, the emphasis of this report is on identifying matchups between the energy available in waste heat streams and the thermal energy inputs to process operations. No attempt has been made to evaluate how much energy loss would take place in transferring energy from waste heat sources to energy input uses. This thermodynamic analys is must be done in conjunction with specific heat recovery and transfer technology assessments.

This chapter discusses the selection of the temperature ranges used to assess the applicability of energy recovery. The compilation of energy stream data by temperature range for 3 types of waste heat streams, 40 generic types of process equipment, and 108 industrial processes is presented. Matchup of waste and input heat streams is also addressed.

\section{SELECTION OF TEMPERATURE RANGES}

One key factor in assessing the applicability of energy recovery technology is the temperature difference between the waste heat stream and process input stream (the source and sink, respectively). The industrial energy flow 
data base associates specific temperatures with energy streams. Waste heat streams can have temperatures from slightly above ambient to over $2000^{\circ} \mathrm{F}$. Energy streams at ambient conditions are assumed to have a temperature of $75^{\circ} \mathrm{F}$. Researchers involved in assessing the applicability of advanced energy recovery indicated that the full spectrum of waste heat temperatures should be subdivided.

Sixteen temperature ranges were selected for this assessment. The 16 ranges span the temperature spectrum from $80^{\circ} \mathrm{F}$ to $2300+{ }^{\circ} \mathrm{F}$. Table 5.1 identifies the temperatures associated with each range. Temperature ranges at the lower end of this spectrum are relatively small compared to those at the higher temperature end of the spectrum. After the industry energy flow data were evaluated with participants in DOE's Chemical Heat Pump Program, it was agreed that the same temperature ranges for classifying waste heat stream temperatures should also be applied to input stream temperatures.

TABLE 5.1 Waste Heat/Input Energy Temperature Ranges

\begin{tabular}{cc} 
Temperature Range & Degrees Fahrenhe \\
\cline { 2 - 3 } 1 & $80-119$ \\
2 & $120-139$ \\
3 & $140-211$ \\
4 & $212-249$ \\
5 & $250-299$ \\
6 & $300-349$ \\
7 & $350-399$ \\
8 & $400-499$ \\
9 & $500-599$ \\
10 & $600-799$ \\
11 & $800-999$ \\
12 & $1000-1199$ \\
13 & $1200-1499$ \\
14 & $1500-1399$ \\
15 & $1900-2299$ \\
16 & $2300+$
\end{tabular}




\section{ENERGY STREAM DATA}

In this section, heat stream data by temperature range are presented in three different ways: by type of waste stream (liquid and gaseous streams combined, liquid only, and gaseous only), by generic type of equipment ( 40 types), and by industrial process (two examples).

\section{Compiled by Type of Waste Stream}

The annual energy content for the waste heat streams and for the energy input streams within each of the 16 temperature ranges was compiled for each of the 108 industrial processes. Three classes of streams were compiled: liquid and gaseous waste and input heat streams combined, only liquid waste and input heat streams, and only gaseous waste and input heat streams. The separation of these streams into three classes can be useful for specific technology applications. Table 5.2 summarizes for all 108 processes the estimated energy content of heat streams in each of the 16 temperature ranges. Estimated values in trillions of Btu are shown for all these classes. The waste heat streams are grouped together as are the process input heat streams. As expected, the majority of the liquid waste heat streams are in the three lowest temperature ranges, whereas gaseous waste heat streams span the full temperature range spectrum.

TABLE 5.2 Energy Content of Heat Streams by Temperature Range

\begin{tabular}{l} 
Temperature \\
Range F \\
\hline $80-119$ \\
$120-139$ \\
$140-211$ \\
$212-249$ \\
$250-299$ \\
$300-349$ \\
$350-399$ \\
$400-499$ \\
$500-599$ \\
$600-i 99$ \\
$800-999$ \\
$1000-1199$ \\
$1200-1499$ \\
$1500-1899$ \\
$1900-2299$ \\
$2300-+$
\end{tabular}

\begin{tabular}{|c|c|c|}
\hline $\begin{array}{l}\text { Liquid \& } \\
\text { Gaseous } \\
\end{array}$ & Liquid & Gaseous \\
\hline $2,522.0$ & 2473.1 & 48.9 \\
\hline 124.7 & 100.6 & 24.2 \\
\hline 533.2 & 337.9 & 195.3 \\
\hline 562.3 & 9.5 & 552.8 \\
\hline 103.5 & 0.0 & 103.5 \\
\hline 299.4 & 1.1 & 298.3 \\
\hline 140.3 & 0.3 & 140.0 \\
\hline 597.6 & 0.0 & 597.6 \\
\hline 191.5 & 0.0 & 191.5 \\
\hline 571.1 & 0.0 & 571.1 \\
\hline 269.7 & 0.0 & 269.7 \\
\hline 119.6 & 0.0 & 119.6 \\
\hline 327.2 & 0.0 & 327.2 \\
\hline 115.3 & 0.0 & 115.3 \\
\hline 192.5 & 0.0 & 192.5 \\
\hline 0.2 & 0.0 & 0.2 \\
\hline
\end{tabular}

\begin{tabular}{|c|c|c|}
\hline $\begin{array}{l}\text { Liquid \& } \\
\text { Gaseous }\end{array}$ & Liquid & Gaseous \\
\hline 29.7 & 0.0 & 29.7 \\
\hline 6.1 & 6.1 & 0.0 \\
\hline 48.7 & 48.7 & 0.0 \\
\hline 53.1 & 1.4 & $5 i .6$ \\
\hline 1244.0 & 0.0 & 1244.0 \\
\hline 420.8 & 0.0 & 420.8 \\
\hline 228.00 & 23.3 & 205.3 \\
\hline 592.1 & 0.0 & 592.1 \\
\hline 80.8 & 0.0 & 80.8 \\
\hline 137.1 & 0.0 & 137.1 \\
\hline 81.1 & 0.0 & 81.1 \\
\hline 119.3 & 0.0 & 119.3 \\
\hline 65.8 & 0.0 & 65.8 \\
\hline 124.6 & 0.0 & 124.6 \\
\hline 0.0 & 0.0 & 0.0 \\
\hline 0.0 & 0.0 & 0.0 \\
\hline
\end{tabular}


Compiled by Type of Equipment

The annual energy content of gas, liquid and steam waste heat streams were compiled for 40 types of process equipment. The results of the combined liquid and gaseous waste heat streams are shown in Table 5.3. Distillation (727.8) and chemical reactors $(334.6)$ have very large waste heat streams in the $80-$ $119^{\circ} \mathrm{F}$ temperature range. Boiler waste stream temperatures center around 400$499^{\circ} \mathrm{F}$, whereas furnace waste stream temperatures are more spread out because of the use of combustion preheat in some industries.

Compiled by Process

Table 5.4 provides an example of the data-base information compiled for a single process (2011-1 Meat Packing). In this process, the temperature of the combined liquid and gaseous waste heat streams (column 3) falls into five temperature ranges and process input heat (column 4) falls within two temperature ranges. This pattern is typical of many industrial processes. An industrial process seldom has more than 7 waste heat temperature ranges or more than 3 input heat temperature ranges. The total annual energy content of the liquid and gaseous waste heat streams (column 3) for this process is estimated to be about 50 trillion Btu. Energy input streams to the meat packing process include direct use of electricity and fossil fuels, as well as heated liquids, heated gases and steam. The input energy streams noted in Table 5.4 are only those associated with thermal energy streams--hot liquids, hot gases and steam. Table 5.5 provides another example of waste heat and input heat stream data, this time for industrial process 2611-1, Pulp Mills (Kraft). Similar data were compiled for all 108 industrial processes.

\section{MATCHUP OF WASTE AND INPUT HEAT STREAMS}

- As mentioned at the beginning of this chapter, the utility of any energy recovered from waste heat streams depends largely on the immediate energy input needs of unit operations within the process. Table 5.4 indicates that industry process 2011-1 contains waste heat streams in 5 temperature categories, 80-119, 120-139, 212-249, 400-499 and 500-599. The energy content of these streams can be used to displace the process input heat needs in the temperature ranges of 140-211 and 250-299. Table 5.6 shows the waste heat (output energy) values for 
TABLE 5.3 Waste Heat Energy By Type of Equipment - $10^{12} \mathrm{Btu} / \mathrm{Yr}$

\begin{tabular}{|c|c|c|c|c|c|c|c|c|c|c|c|c|c|c|c|c|c|}
\hline rype of Process Equiprent & $\begin{array}{l}80- \\
119^{\circ} \mathrm{F}\end{array}$ & $\begin{array}{l}120- \\
139^{\circ} \mathrm{F}\end{array}$ & $\begin{array}{l}140- \\
211^{\circ} \mathrm{F}\end{array}$ & $\begin{array}{l}212- \\
249^{\circ} \mathrm{F} \\
\end{array}$ & $\begin{array}{l}250- \\
299^{\circ} \mathrm{F}\end{array}$ & $\begin{array}{l}300- \\
349^{\circ} \mathrm{F} \\
\end{array}$ & $\begin{array}{l}350- \\
399^{\circ} \mathrm{F} \\
\end{array}$ & $\begin{array}{l}400- \\
499^{\circ} \mathrm{F}\end{array}$ & $\begin{array}{l}500- \\
599^{\circ} \mathrm{F} \\
\end{array}$ & $\begin{array}{l}600- \\
799^{\circ} \mathrm{F}\end{array}$ & $\begin{array}{l}800- \\
999^{\circ} \mathrm{F} \\
\end{array}$ & $\begin{array}{l}1000- \\
1199^{\circ} \mathrm{F}\end{array}$ & $\begin{array}{l}1200- \\
1499^{\circ} \mathrm{F}\end{array}$ & $\begin{array}{l}1500- \\
1899^{\circ} \mathrm{F}\end{array}$ & $\begin{array}{l}1900- \\
2299^{\circ} \mathrm{F}\end{array}$ & $\begin{array}{l}2300- \\
U^{\circ} \mathrm{F}\end{array}$ & TOTAL \\
\hline Absorption & 13.5 & & 55.2 & 1.59 & & & & & & & & & & & & & 70.3 \\
\hline Annealing & & & & & 51.0 & & & & & 1.71 & 2.50 & 9.74 & 13.6 & 14.7 & & & 93.3 \\
\hline Boller & & & & & & 0.49 & 0.81 & 447.3 & 74.3 & 51.1 & 1.13 & & & & & & 575.2 \\
\hline Casting. Molding & 3.62 & & 10.4 & 7.69 & & 29.2 & & & 0.84 & & & & & & & & 51.8 \\
\hline Centrifuge & 0.47 & 0.02 & 0.60 & & & & & & & & & & & & & & 1.1 \\
\hline Coking & 27.9 & & & & & & & & & & & 6.47 & & & & & 34.4 \\
\hline Compressor & 81.9 & & 0.56 & & & & & & & & & & & & & & 82.5 \\
\hline Condenser & 50.9 & 0.21 & 0.29 & 1.65 & & & & & & & & & & & & & 53.0 \\
\hline Cooking & & & 0.16 & 2.94 & & & & 5.41 & & & & & 9.65 & & & & 18.2 \\
\hline Cooling & 89.1 & 54.3 & 43.5 & 1.08 & 0.81 & 1.85 & 0.18 & & 17.3 & & & 0.04 & & & & & 208.2 \\
\hline Crushing, Grinding & 16.0 & & & & & & & & & & & & & & & & 16.0 \\
\hline Crystallization & 6.31 & & 0.58 & & & & & & & & & & & & & & 6.9 \\
\hline Cutting, Trinuing & 1.01 & & & & & & & & & & & & & & & & 1.0 \\
\hline Distillation & 727.8 & & 0.28 & 0.30 & & & & & & 68.8 & 142.5 & & & & & & 939.7 \\
\hline Drying. & 158.1 & & 113.8 & 215.1 & 13.5 & 11.2 & 106.6 & 57.4 & 0.16 & 92.3 & & 10.2 & & & & & 778.4 \\
\hline Electric Generation & 185.2 & 37.1 & 13.6 & & & & 0.73 & 1.25 & 0.20 & 119.9 & 0.09 & & & & & & 358.1 \\
\hline Electrolytic Cells & & & & & & & & & & & & 0.06 & & 26.9 & & & 27.0 \\
\hline Evaporation & 189.2 & 0.27 & 119.3 & 0.48 & & & & & & & & & & & & & 309.2 \\
\hline Extraction & 3.52 & 8.12 & & & & & & & & & & 1.08 & & & & & 12.7 \\
\hline Extruding & 0.77 & & & & & & & & & & & & & & & & 0.8 \\
\hline filter & 5.71 & & 1.00 & & & & & & & & 2.37 & & & & & & 9.1 \\
\hline
\end{tabular}


TABLE 5.3. (contd)

\begin{tabular}{|c|c|c|c|c|c|c|c|c|c|c|c|c|c|c|c|c|c|}
\hline Type of Process Equipment & $\begin{array}{l}80- \\
119^{\circ} \mathrm{F}\end{array}$ & $\begin{array}{l}120- \\
139^{\circ} \mathrm{F}\end{array}$ & $\begin{array}{l}140- \\
211^{\circ} \mathrm{F}\end{array}$ & $\begin{array}{l}212- \\
249^{\circ} \mathrm{F} \\
\end{array}$ & $\begin{array}{l}250- \\
299^{\circ} \mathrm{F} \\
\end{array}$ & $\begin{array}{l}300- \\
349^{\circ} \mathrm{F} \\
\end{array}$ & $\begin{array}{l}350- \\
399^{\circ} \mathrm{F} \\
\end{array}$ & $\begin{array}{l}400- \\
499^{\circ} \mathrm{F} \\
\end{array}$ & $\begin{array}{l}500- \\
599^{\circ} \mathrm{F}\end{array}$ & $\begin{array}{l}600- \\
799^{\circ} \mathrm{F} \\
\end{array}$ & $\begin{array}{l}800- \\
999^{\circ} \mathrm{F}\end{array}$ & $\begin{array}{l}1000- \\
1199^{\circ} \mathrm{F} \\
\end{array}$ & $\begin{array}{l}1200- \\
1499^{\circ} \mathrm{F}\end{array}$ & $\begin{array}{l}1500- \\
1899^{\circ} \mathrm{F}\end{array}$ & $\begin{array}{l}1900- \\
2299^{\circ} \mathrm{F}\end{array}$ & $\begin{array}{l}2300- \\
U P^{\circ} \mathrm{F} \\
\end{array}$ & IOIAL \\
\hline Flash Separator & 0.02 & & 0.22 & 1.14 & & & & & & & & & & & & & 1.4 \\
\hline Furnace & 168.4 & & 12.5 & & 19.4 & 204.5 & & 13.3 & 79.2 & 44.5 & 49.4 & 91.7 & 296.6 & 64.4 & 173.7 & & $1,217.4$ \\
\hline Heat Exchangers & 79.8 & & 4.69 & 0.23 & & & & & & & 0.65 & & & & & & 85.3 \\
\hline Heating & 27.6 & & & 2.69 & 18.8 & 4.65 & 4.47 & 6.05 & 3.43 & 11.2 & 0.89 & 0.06 & & 7.03 & & 0.20 & 93.1 \\
\hline Leaching & 11.5 & & 1.93 & & & & & & 0.03 & & & & & & & & 13.5 \\
\hline Major Mech. Proc. & 0.75 & & 0.10 & & & & & & & & & & & & & & 0.8 \\
\hline Misc. Mechanical & 1.41 & 9.00 & 21.7 & 5.11 & & & & 1.84 & & 2.04 & & & & & & & 47.1 \\
\hline Mixing & 0.51 & 0.31 & 5.51 & & & 1.50 & & & & & & & & & & & 1.8 \\
\hline Ovens & & 0.26 & 74.0 & 33.6 & 0.01 & 0.53 & 24.2 & 18.0 & 0.56 & 77.4 & 22.2 & & 2.12 & & 6.61 & & 259.5 \\
\hline Packaying & 2.28 & & & 0.08 & 0.08 & & & & & & & & & & & & 2.4 \\
\hline Pasteurizing & & & 7.17 & 1.11 & & & & & & & & & & & & & 8.3 \\
\hline Prine Mover, Mech. & 73.1 & & 7.97 & & & . & & & 2.22 & 0.54 & & & & & & & 83.8 \\
\hline Reactors & 334.6 & & 22.6 & 4.31 & & 0.23 & 3.26 & 46.0 & 10.4 & 4.26 & 47.9 & & 0.45 & & 12.3 & & 486.2 \\
\hline Rolling & 1.02 & & & 154.8 & & & & & & 1.98 & & & & & & & 157.8 \\
\hline Separation & 64.2 & & 2.03 & 0.66 & & 1.51 & & & & & & & & & & & 68.4 \\
\hline Settling & & & 2.14 & & & & & & & & & 0.25 & & 0.66 & & & 3.0 \\
\hline Storage, Holding Tank & & & & 126.9 & & & & & & & & & & & & & 126.9 \\
\hline Stripping & 97.3 & & & & & 37.5 & & & & & & & & & & & 134.8 \\
\hline Washing & 2.33 & 15.1 & 9.18 & & & & & & & & & & & & & & 26.6 \\
\hline otlier & 90.10 & & 2.29 & 0.91 & & 6.18 & & 0.97 & 2.89 & 89.28 & & & 4.76 & 1.58 & & & 199.0 \\
\hline TOTAL & $2,522.0$ & 124.7 & 533.2 & 562.3 & 103.5 & 299.4 & 140.3 & 597.6 & 191.5 & 534.1 & 269.7 & 119.6 & 327.2 & 115.3 & 192.5 & 0.20 & 6.670 .1 \\
\hline
\end{tabular}


TABLE 5.4. Energy Content of Waste Heat and Input Heat Streams for Industrial Process 2011-1, Meat Packing

Temperature Range

$\begin{array}{rc}1 & 80-119 \\ 2 & 120-139 \\ 3 & 140-211 \\ 4 & 212-249 \\ 5 & 250-299 \\ 6 & 300-349 \\ 7 & 350-399 \\ 8 & 400-499 \\ 9 & 500-599 \\ 10 & 600-799 \\ 11 & 800-999 \\ 12 & 1000-1199 \\ 13 & 1200-1499 \\ 14 & 1500-1899 \\ 15 & 1900-2299 \\ 16 & 2300^{+}\end{array}$

$2300^{+}$
Energy Content in Trillions of Btu $\left(10^{12}\right)$ Liquid \& Gaseous Streams Waste Heat Input Heat

\begin{tabular}{|c|c|}
\hline 13.32 & $-\ldots$ \\
\hline 6.58 & --- \\
\hline--- & 8.09 \\
\hline 5.05 & $-\cdots$ \\
\hline$-\ldots$ & 28.49 \\
\hline$-\ldots$ & $\ldots$ \\
\hline$-\cdots$ & $-\cdots$ \\
\hline 3.90 & --- \\
\hline 19.92 & --- \\
\hline--- & -..- \\
\hline--- & $-\cdots$ \\
\hline -- & -- \\
\hline--- & --- \\
\hline--- &.-- \\
\hline--- & $-\cdots$ \\
\hline$\cdots$ & $\ldots$ \\
\hline
\end{tabular}

$$
\begin{array}{r}
13.32 \\
6.58 \\
- \\
- \\
- \\
- \\
-
\end{array}
$$

Gaseous Streams Waste Heat Input Heat$$
-\cdots
$$$$
-
$$$$
8.09
$$$$
---
$$$$
\text { -.-. }
$$$$
5.05
$$$$
\text { -... }
$$ 
TABLE 5.5. Energy Content of Waste Heat and Input Heat Streams for Industrial Process 2611-1, Pulp Mills (Kraft)

Energy Content in Trillions of Btu $\left(10^{12}\right)$

Temperature

Range

1

2

3

4

5

6

7

\begin{tabular}{c} 
Temperature \\
\hline $80-119$ \\
$120-139$ \\
$140-211$ \\
$212-249$ \\
$250-299$ \\
$300-349$ \\
$350-399$ \\
$400-499$ \\
$500-599$ \\
$600-799$ \\
$800-999$ \\
$1000-1199$ \\
$1200-1499$ \\
$1500-1899$ \\
$1900-2299$ \\
$2300^{+}$
\end{tabular}

Liquid \& Gaseous Streams Waste Heat Input Heat

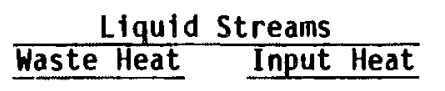

20.40

$\cdots$

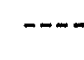

4.46

17.79

-..-

9.60

3.15

$---$

1.64

1.98

$-\cdots$

$-\cdots$

$--\cdot$
Gaseous Streams Waste Heat Input Heat

\begin{tabular}{|c|c|}
\hline 20.40 & $-\ldots$ \\
\hline--- & $-\ldots$ \\
\hline 4.46 & $\ldots$ \\
\hline$\ldots$ & $\cdots$ \\
\hline-- & $-\cdots$ \\
\hline -.-- & $-\infty$ \\
\hline--- & $-\cdots$ \\
\hline -.- & $-\cdots$ \\
\hline -..- & --- \\
\hline--- & $-\cdots$ \\
\hline--- & - \\
\hline--- & -- \\
\hline$-\cdots$ & \\
\hline$\cdots-$ & --- \\
\hline--- & - \\
\hline$\cdots$ & $\rightarrow$ \\
\hline
\end{tabular}

\begin{tabular}{|c|c|}
\hline$\cdots$ & $-\cdots$ \\
\hline-- & $\ldots$ \\
\hline-- & --- \\
\hline 17.79 & -.- \\
\hline--- & 37.32 \\
\hline 9.60 & 10.18 \\
\hline--- & --- \\
\hline 3.15 & $-\ldots$ \\
\hline.-- & --- \\
\hline 1.64 & -- \\
\hline 1.98 & --- \\
\hline--- & --- \\
\hline$\cdots$ & --- \\
\hline$-\cdots$ & --- \\
\hline-- & \\
\hline$-\ldots$ & \\
\hline
\end{tabular}


TABLE 5.6. Liquid and Gaseous Waste Heat Stream Utility Matrix Process 2011-1, Meat Packing

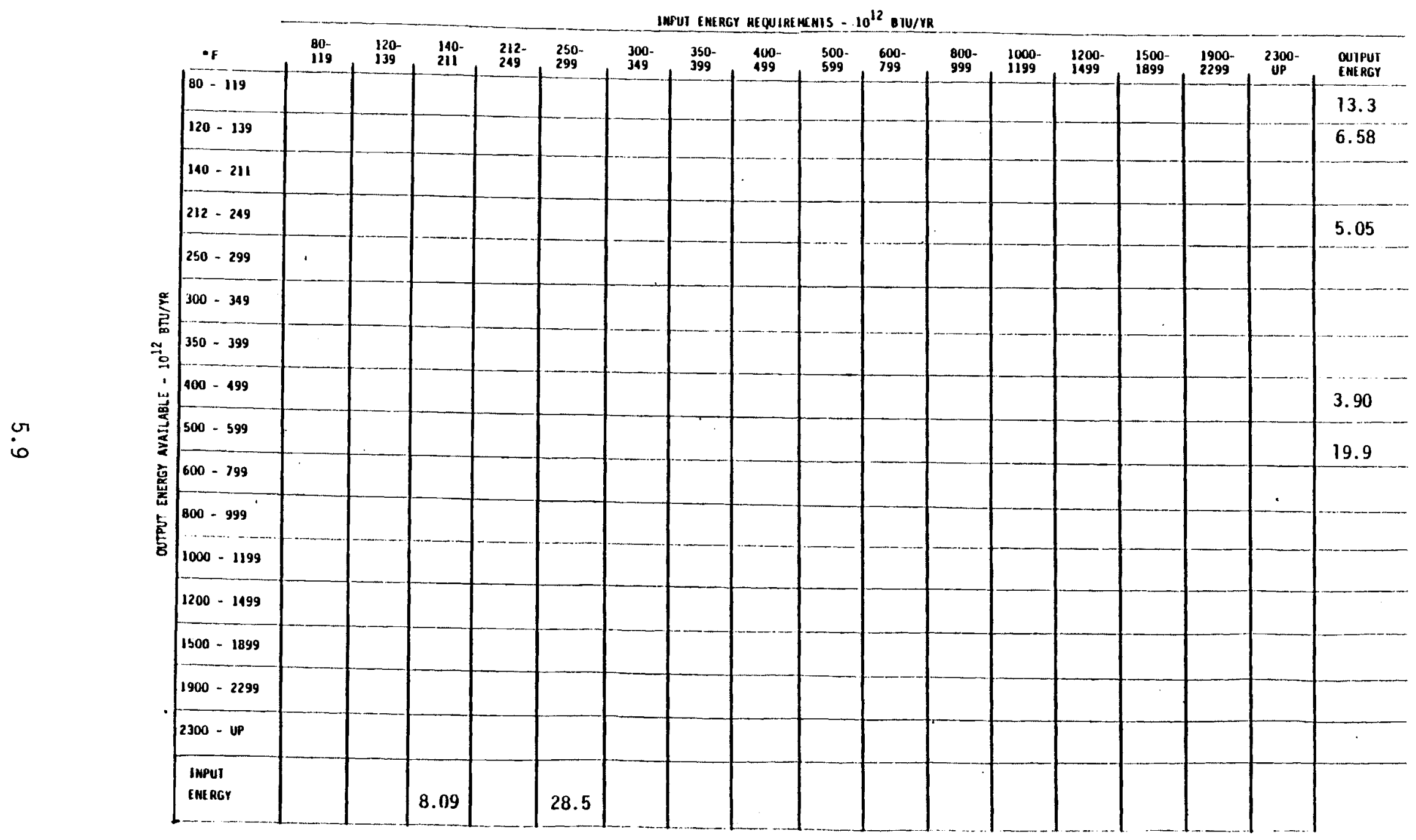


each of 5 temperature ranges in the right hand column, and the two input heat (input energy) values in the bottom row. In Table 5.6, no attempt has been made to match source (output energy) with sink (input energy) values. In Table 5.7 the output energy for temperature range $80-119^{\circ} \mathrm{F}\left(13.3 \times 10^{12} \mathrm{Btu}\right)$ is compared to the need for heat energy in the $140-211^{\circ} \mathrm{F}$ temperature range $(8.09$ $\times 10^{12} \mathrm{Btu}$ ) and the "input energy" value (the lesser of the two) selected for the cell. The $8.09 \times 10^{12}$ Btu value was placed in the cell because that is the maximum amount of heat required at temperature range $140-211^{\circ} \mathrm{F}$ by the process. Table 5.8 next compares the $80-119^{\circ} \mathrm{F}$ output energy value $(13.3 \mathrm{x}$ $\left.10^{12} \mathrm{Btu}\right)$ to the input energy requirement for the $250-299^{\circ} \mathrm{F}$ temperature range $\left(28.5 \times 10^{12} \mathrm{Btu}\right)$. This time the input energy requirements $(28.5 \times$ $\left.10^{12} \mathrm{Btu}\right)$ exceed the output energy $\left(13.3 \times 10^{12} \mathrm{Btu}\right)$ and the 13.3 value is placed in the cell. Table 5.9 shows the utility matrix completed for process 2011-1. The 5 output energy temperature ranges intersect the 2 input energy temperature ranges in 10 of the matrices 256 cells. In cells where an output energy and input energy value intersect the smaller of the 2 values is entered.

Table 5.10 presents the utility matrix for Process 2611-1 (Pulp Mills, Kraft). This process contains waste heat streams that fall within 7 temperature ranges, and input heat requirements that fall within 2 temperature ranges. Note that the full amount of the energy value in the output streams for temperature ranges $140-211^{\circ} \mathrm{F}, 300-349^{\circ} \mathrm{F}, 400-499^{\circ} \mathrm{F}, 600-799^{\circ} \mathrm{F}$, and $800-999^{\circ} \mathrm{F}$ is available to both of the input energy temperature ranges. No attempt is made in these utility matrices to prioritize how waste heat should be used.

The utility value is the maximum amount of input heat energy that could be displaced given $100 \%$ efficiency in transfering energy from source to sink. (0f course, the laws of thermodynamics prevents this. These values should be adjusted based on the efficiency of the heat recovery equipment being assessed.) 
TABLE 5.7. Liquid and Gaseous Waste Heat Stream Utility Matrix Process 2011-1 Meat Packing

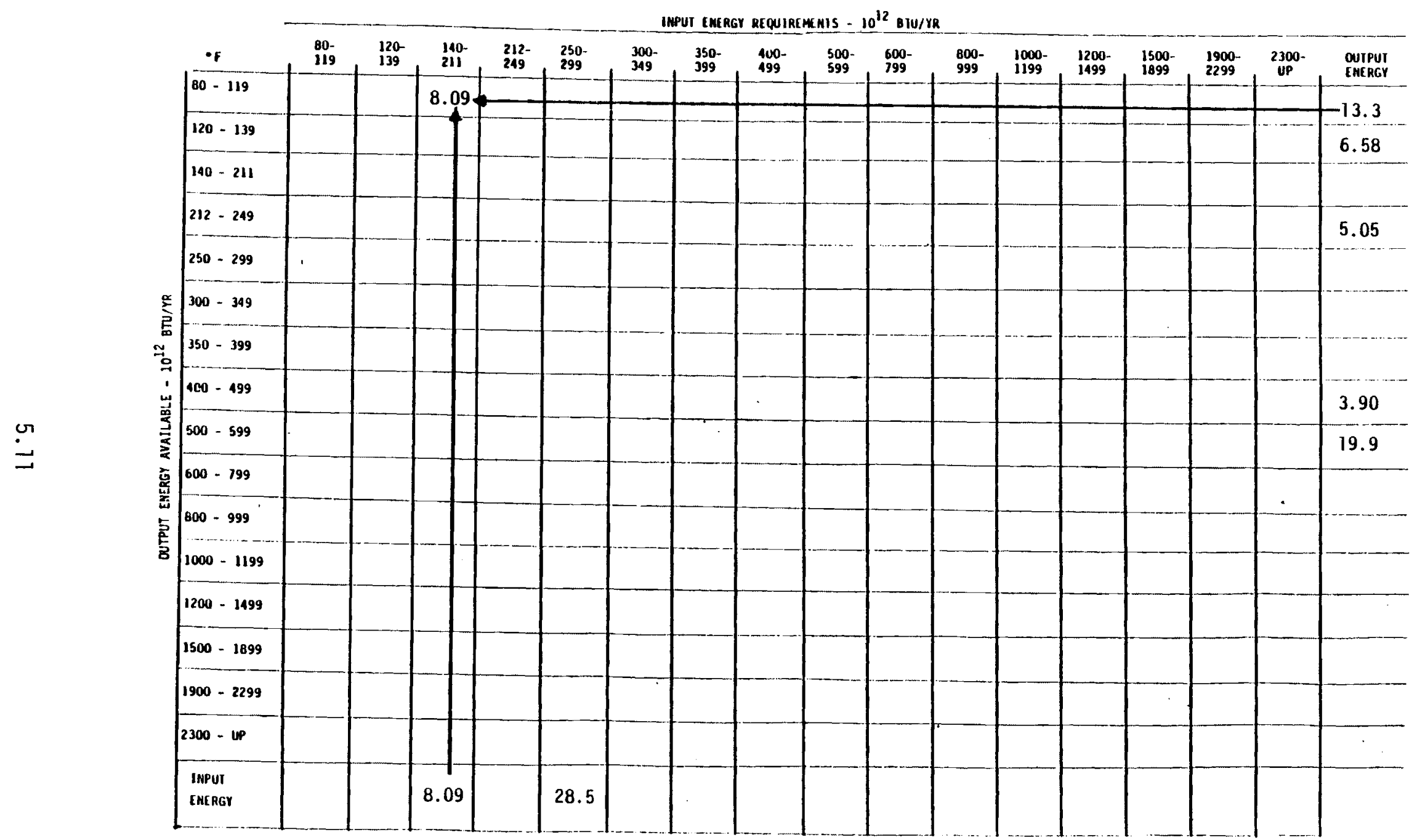


IABLE 5.8. Liquid and Gaseous Waste Heat Stream Utility Matrix Process 2011-1, Meat Packing

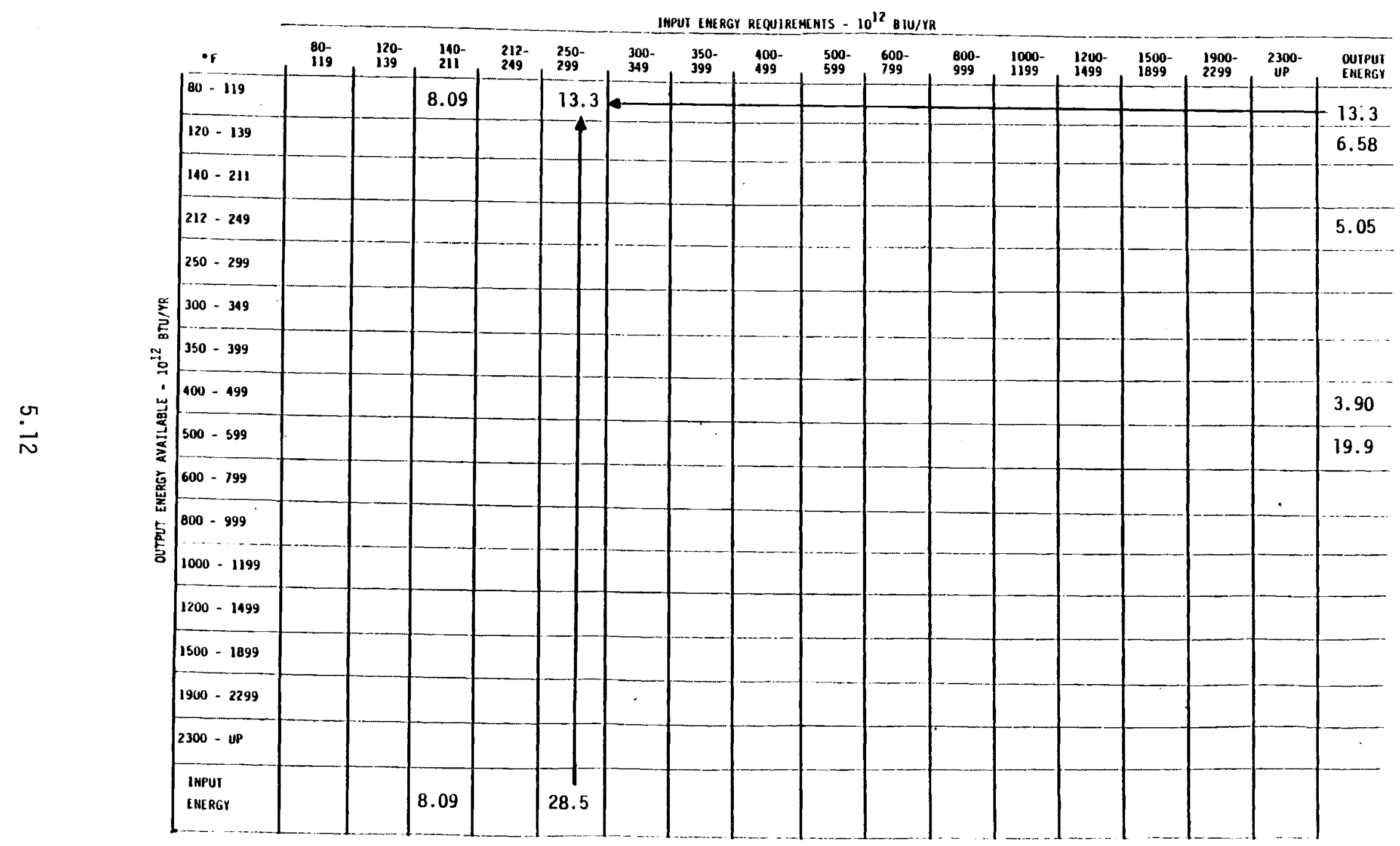


TABLE 5.9. Liquid and Gaseous Waste Heat Stream Utility Matrix -
Process 2011-1, Meat Packing

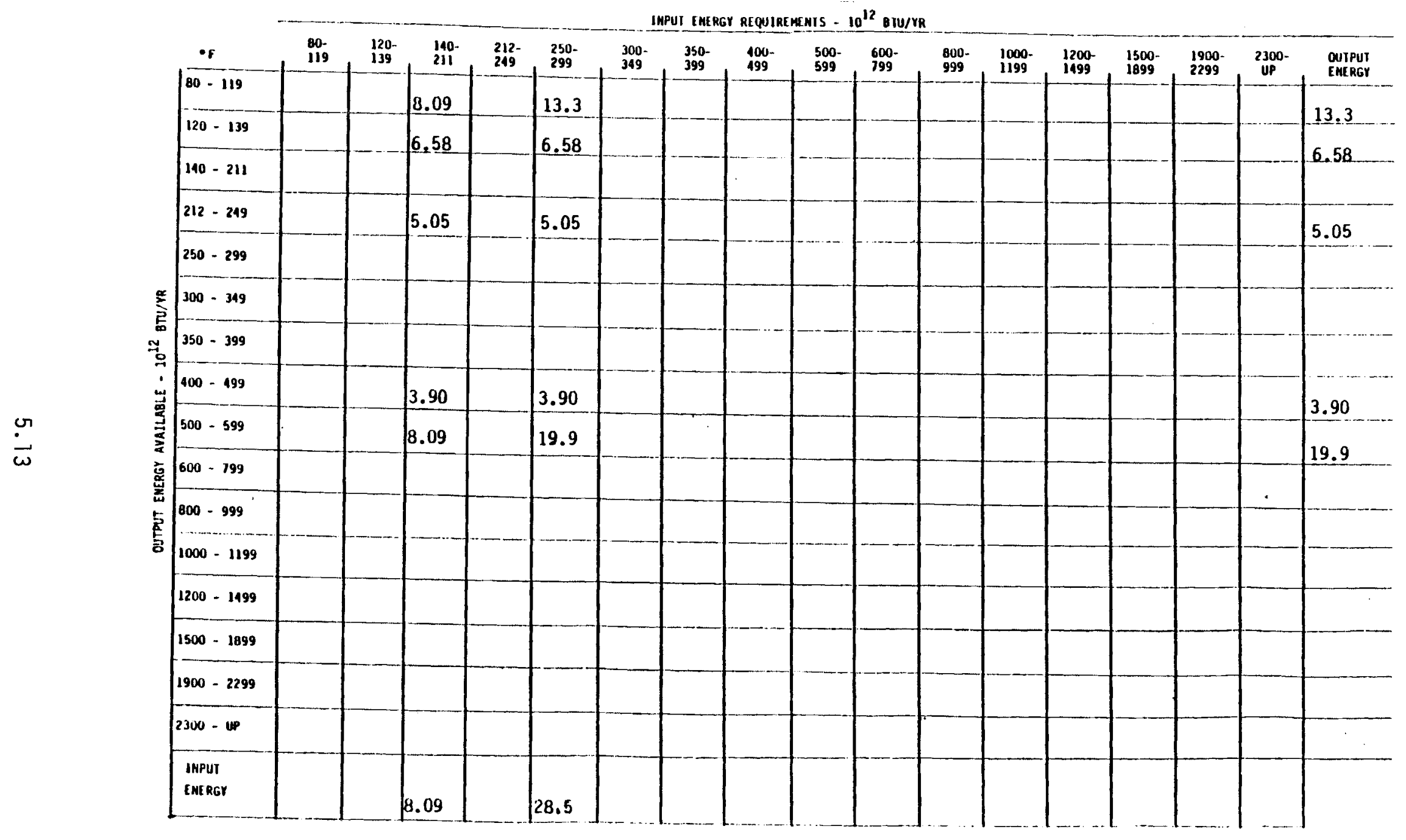


IABLE 5.10. Liquid and Gaseous Waste Heat Stream Utility Matrix Process 2611-1, Pulp Mills (Kraft)

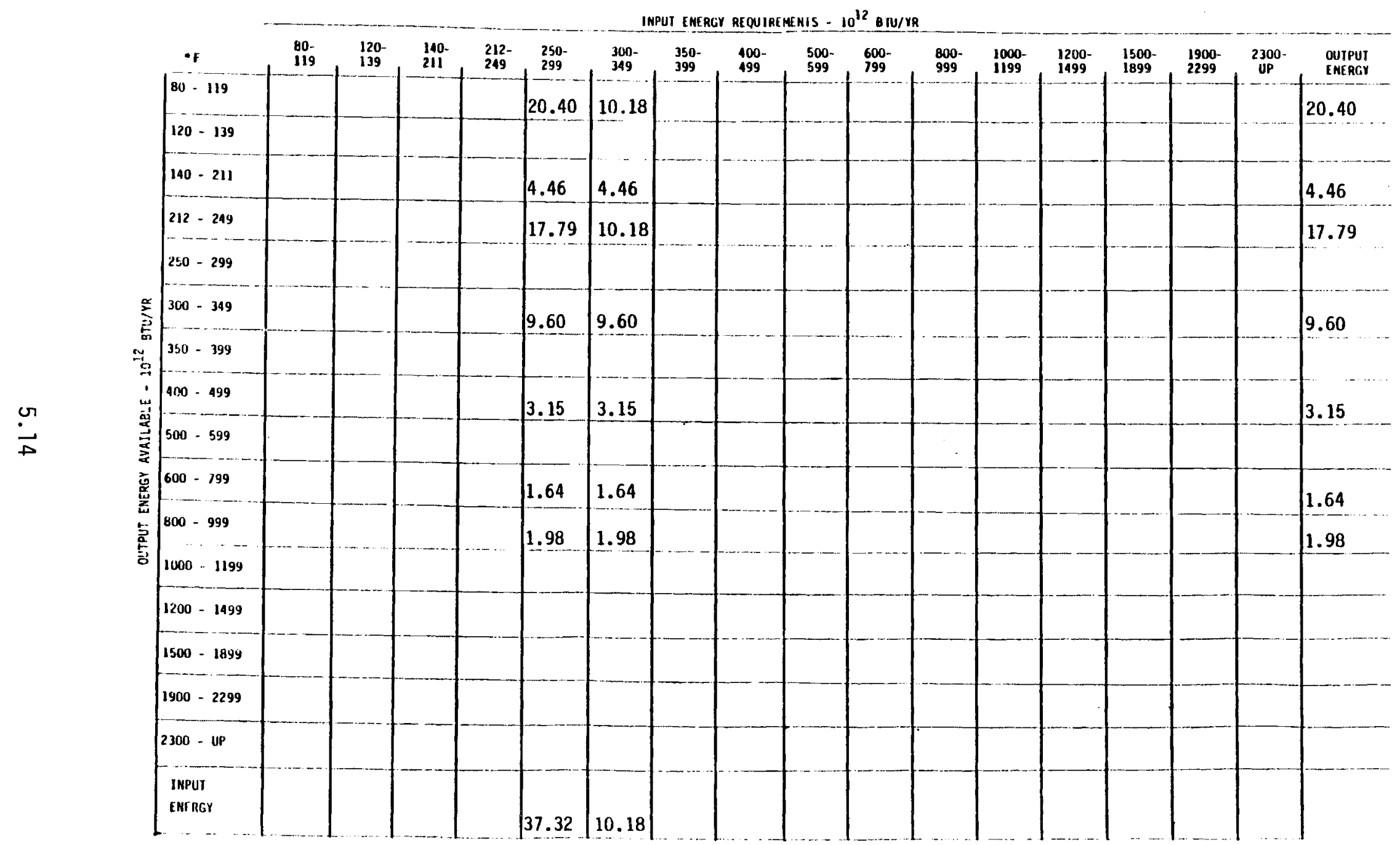




\subsection{WASTE HEAT RECOVERY OPPORTUNITIES}

Chapter 5.0 described the maximum amount of energy (per year) transferable from energy sources to energy uses in each of 108 industrial processes and the method used to determine source/sink matchup values. For individual industry processes, an energy matchup value was tabulated for each source/sink temperature combination and placed in a 256 cell matrix (defined as a utility matrix). This chapter summarizes the data from all 108 industrial processes addressed and indicates the amount of energy in waste heat streams that can be potentially used. Summary tables of source/sink matchups represent opportunities for waste heat recovery applications across the manufacturing sector of the U.S. economy. In Chapter 5.0 the maximum transferable energy determined from source/sink matchups (comparisons) was presented in utility matrices for specific industrial processes. A separate process utility matrix was developed for each of the 108 industrial processes evaluated.

To summarize the Chapter 5.0 utility matrix data across all 108 industrial processes, the individual matrix values for each source/sink temperature combination were added. For example, a review of all 108 utility matrices indicates that 11 of the 108 industrial processes had utility matrix values for the source/sink temperature combination defined by $80-119^{\circ} \mathrm{F}$ source and 140 $211^{\circ} \mathrm{F}$ sink. These 11 industries are shown in Table 6.1. Figure 6.1 shows how these 11 values were summed and entered into the liquid and gaseous heat stream summary table. This process was repeated for all 256 source/sink temperature combinations in the utility matrices.

The summary of the 108 industrial processes for the liquid and gas waste heat streams combined, gas only waste heat streams, and liquid only waste heat streams are shown in Tables $6.2,6.3$ and 6.4 , respectively. Remember that in the industry-specific utility matrices in Chapter 5.0 no attempt was made to apportion the output energy among the input energy streams. The objective was to identify the temperature range matchups that offer the largest energy savings potential. The total energy content of an output stream is therefore assumed to be available to any of the input streams. Therefore, it is 
TABLE 6.1 Process Utility Matrix Values for Liquid and Gaseous Waste Heat Streams (source/sink temperature combination $80^{\circ} \mathrm{F}$ to $119^{\circ} \mathrm{F} /$ $140^{\circ} \mathrm{F}$ to $217^{\circ} \mathrm{F}$ )

\begin{tabular}{c} 
Process \# \\
\hline $2011-1$ \\
$2033-1$ \\
$2033-2$ \\
$2033-3$ \\
$2062-1$ \\
$2063-1$ \\
$2082-1$ \\
$2822-2$ \\
$2823-2$ \\
$2899-2$ \\
$3861-1$
\end{tabular}

\begin{tabular}{l} 
Industry Name \\
\hline Meat packing \\
Canned fruits \\
Canned vegetables \\
Fruit and vegetable juices \\
Cane sugar refining \\
Beet sugar refining \\
Malt beverages \\
Synthetic rubber (Butyl) \\
Celluloisic man-made fibers (Acetate) \\
Chemical preparations (fatty acids) \\
Photographic film
\end{tabular}

\begin{tabular}{r}
$\begin{array}{r}\text { Cell Utility } \\
\left.\text { Value (Btu } \times 10^{2}\right)\end{array}$ \\
\hline 8.1 \\
0.50 \\
1.2 \\
0.38 \\
9.3 \\
2.7 \\
13.1 \\
0.14 \\
0.12 \\
0.010 \\
0.014 \\
35.6
\end{tabular}

inappropriate to sum the energy content in rows or columns in either the utility matrices of Chapter 5.0 or in the summary tables presented in Tables 6.2, 6.3, and 6.4.

SUMMARY OF SOURCE/SINK MATCHUPS

Within the temperature range region discussed earlier, waste heat streams and candidate use temperature streams were each grouped into 16 temperature ranges. A total of 256 energy source/sink temperature combinations are possible. Table 6.2 presents a summary across all 108 industrial processes of the energy matchups between potential energy available from waste streams and potential utilization from candidate unit operations. The values in Table 6.2 reflect two basic constraints: 1) the energy available in waste heat streams is not transferred between industrial sites nor industrial processes, and 2) only waste heat energy that can be matched to a candidate use is counted.

As discussed earlier, the total energy content of any waste heat stream is available to any candidate use within an industrial process. Until a specific use actually taps an energy source, the energy source is available to any use. 
IABLE 6.2 Sumuary of 108 Source/Sink Matchups: Combined Liquid and Gaseous Heat Streams

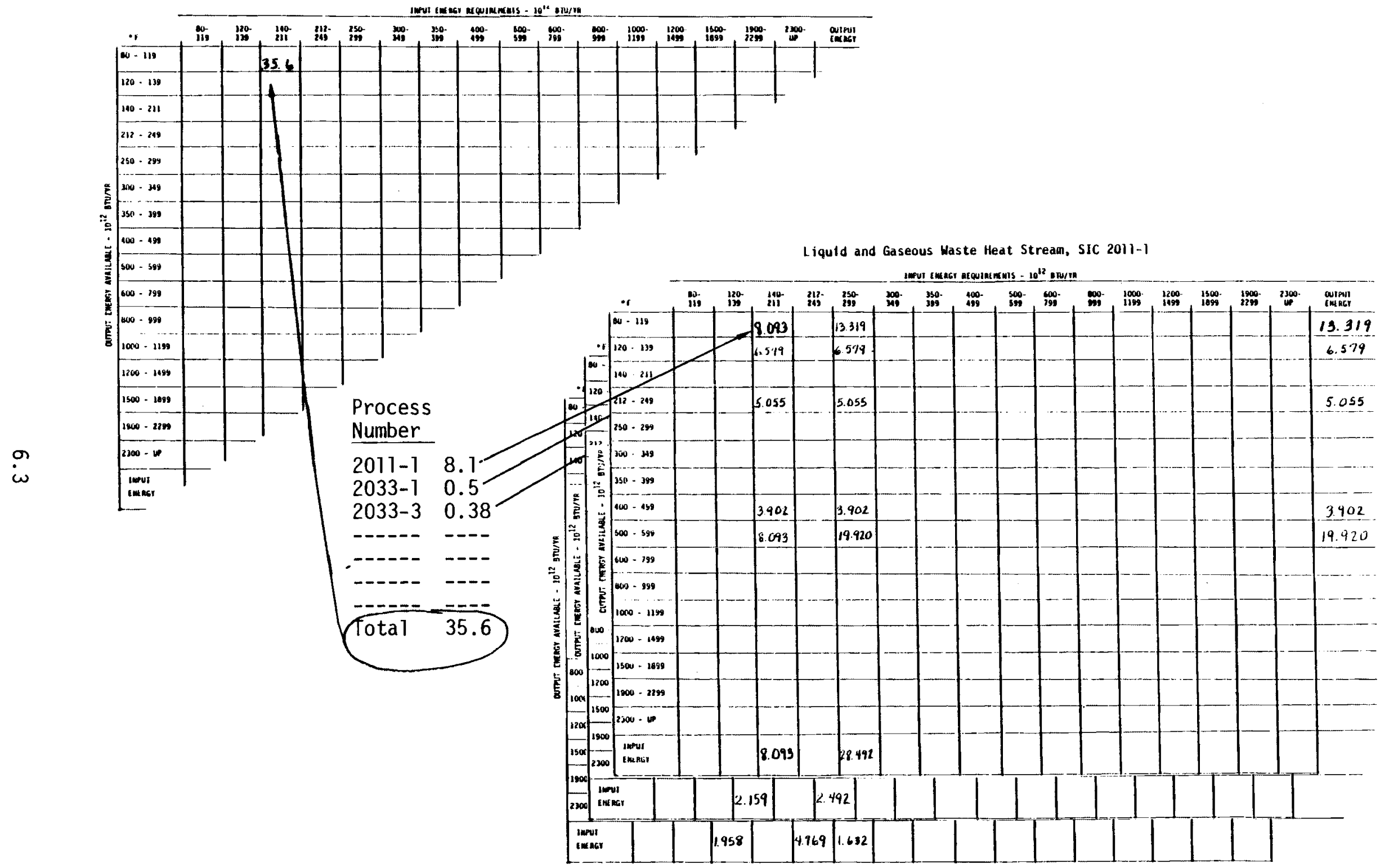

FIGURE 6.1. Development of the Source/Sink Summation Table

(cell values are the lesser of the input \& output energy) 
TABLE 6.2 Summary of 108 Source/Sink Matchups: Combined Liquid and Gaseous Heat Streams

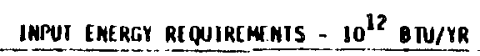

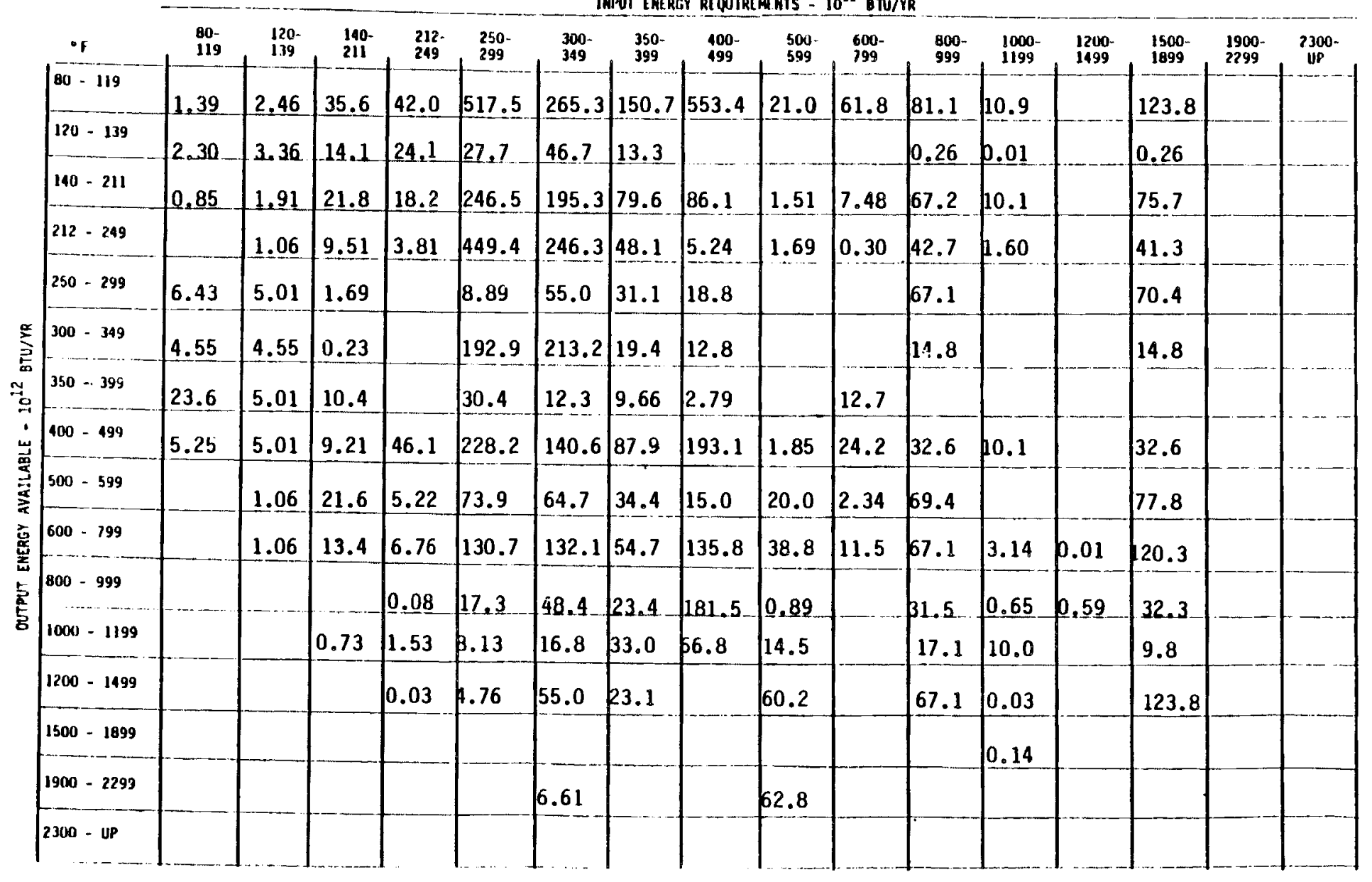


TABLE 6.3 Summary of 108 Source/Sink Matchups: All Gas Heat Streams

\begin{tabular}{|c|c|c|c|c|c|c|c|c|c|c|c|c|c|c|c|c|}
\hline & & & & & & & APUT IMER & IY REQUIRE & MENIS - I & $0^{12}$ BIU & & & & & & \\
\hline - $\mathrm{F}$ & $\begin{array}{l}80- \\
119\end{array}$ & $\begin{array}{l}120- \\
139 \\
\end{array}$ & $\begin{array}{l}140- \\
211\end{array}$ & ${ }_{249}^{212-}$ & $\begin{array}{l}250- \\
299\end{array}$ & $\begin{array}{l}300- \\
349\end{array}$ & $\begin{array}{l}350- \\
399\end{array}$ & 400. & $\begin{array}{l}500- \\
599\end{array}$ & $\begin{array}{l}600- \\
799\end{array}$ & $\begin{array}{l}800- \\
999\end{array}$ & $\begin{array}{l}1000- \\
1199\end{array}$ & $\begin{array}{l}1200- \\
1499\end{array}$ & $\begin{array}{l}1500- \\
1899\end{array}$ & $\begin{array}{l}1900- \\
2299\end{array}$ & $\underset{U P}{2300-}$ \\
\hline $80-119$ & & & & 0.07 & 5.05 & 27.0 & 5.36 & 0.36 & 0.08 & 1.62 & 2.95 & 0.76 & & 2.95 & & \\
\hline $120-139$ & & & & 23.8 & 0.35 & 23.8 & & & & & & 0.01 & & & & \\
\hline $140-211$ & & & & 1.44 & 109.3 & 8.18 & 0.91 & 72.1 & 0.84 & 0.19 & & 0.02 & & & & \\
\hline $212-249$ & & & & 2.37 & 496.6 & 238.6 & 46.5 & 3.80 & 0.66 & 0.30 & 33.6 & & & 33.6 & & \\
\hline $250-299$ & 6.43 & & & & 7.29 & 53.4 & 31.1 & 18.8 & & & 67.1 & & & 70.4 & & \\
\hline $300-349$ & 4.54 & & & & 200.0 & 214.7 & 19.4 & 12.7 & & & 14.8 & & & 14.8 & & \\
\hline $350-399$ & 9.66 & & & & 27.8 & 7.91 & 9.81 & 2.54 & & 12.7 & & & & & & \\
\hline $400-499$ & 5.24 & & & 46.1 & 234.4 & 145.0 & 75.6 & 192.9 & 2.66 & 24.2 & 32.6 & 5.83 & 14.9 & 32.6 & & \\
\hline $500-599$ & & & & 3.78 & 73.2 & 70.7 & 35.0 & 15.0 & 19.5 & 2.34 & 69.4 & & 2.34 & 77.8 & & \\
\hline $600 \cdot 799$ & & & & 6.67 & 132.3 & 126.0 & 53.5 & 135.8 & 40.0 & 11.5 & 64.1 & 29.0 & 0.01 & 120.3 & & \\
\hline $800-999$ & & & & 0.08 & 17.3 & 48.4 & 23.4 & 181.5 & 1.95 & & 31.5 & 0.65 & 0.59 & 31.5 & & \\
\hline $1000-1199$ & & & & 10.3 & 9.21 & 27.0 & 9.75 & 66.8 & 14.5 & & 17.1 & 0.05 & & 9.75 & & \\
\hline $1200-1499$ & & & & 0.04 & 5.84 & 55.0 & 23.1 & & 60.2 & & 67.1 & 0.04 & & 123.8 & & \\
\hline $1500-1899$ & & & & 0.03 & & & & & & & & 0.16 & & & & \\
\hline $1900-2299$ & & & & & 1.07 & 6.61 & & & 62.80 & & & & & & & \\
\hline $2300-4$ & & & & & & & & & & & & & & & & \\
\hline
\end{tabular}


TABLE 6.4 Summary of 108 Source/Sink Matchups: All Liquid Heat Streams

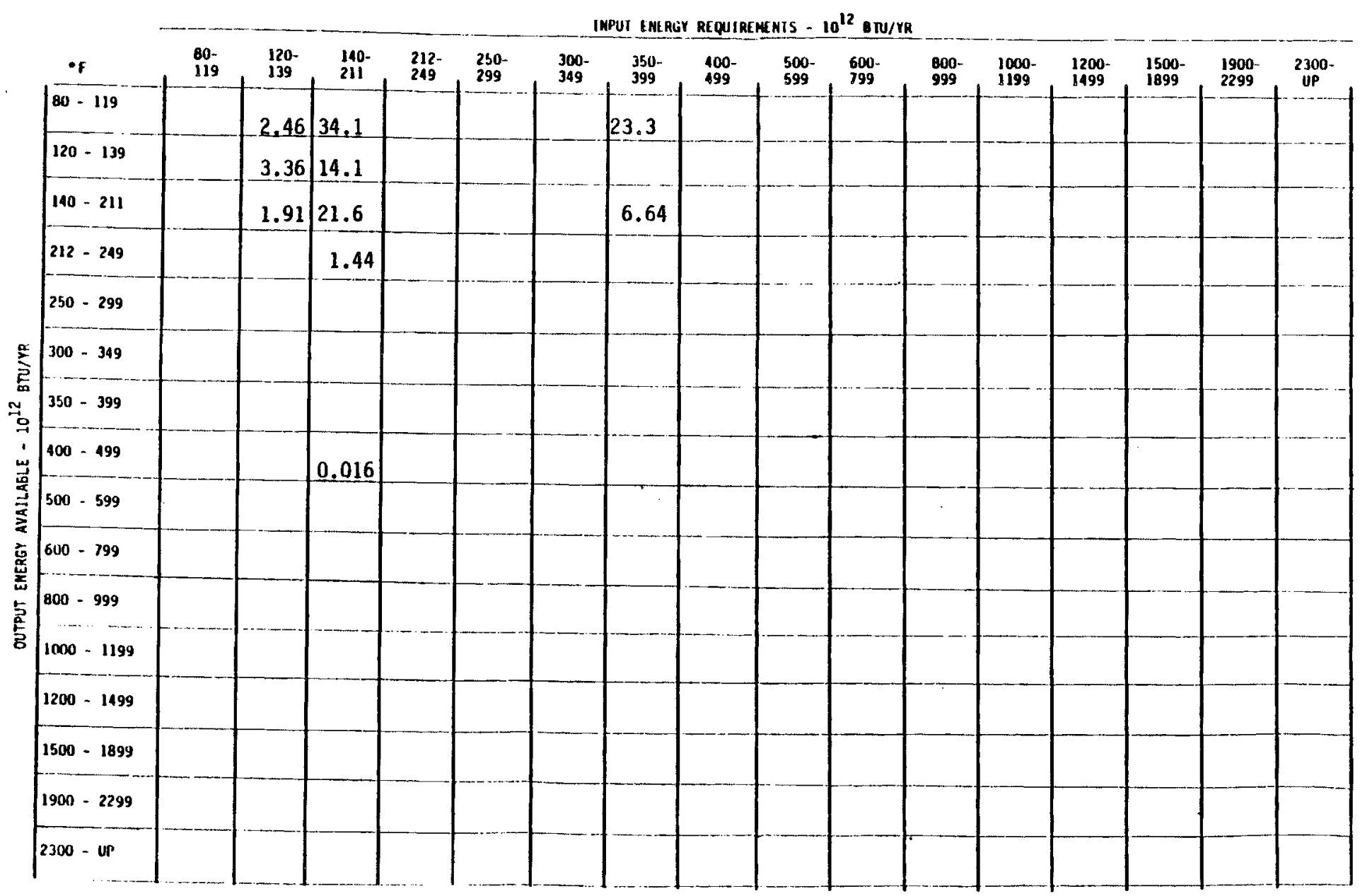


The energy values in Table 6.2 represent the maximum matchup between energy source/energy sink values, and thus the maximum waste heat recovery opportunity. In total, 142 of the 256 matrix cells in Table 6.2 show energy values. Energy values range from 10 billion Btu per year to 553 trillion Btu per year.

Nearly half (116 of 256) of the cells in Table 6.3 have non-zero energy values. The values range from 10 billion to 496.6 trillion Btu per year. In the liquid only heat stream summation table (Table 6.4), 10 of the 256 cells in the table have non-zero values. The number of cells is low because most of the liquid streams are water, which vaporizes at a relatively low temperature.

\section{HEAT BOOSTING/HEAT EXCHANGER OPPORTUNITIES}

Although this task does not address the specific heat recovery technologies currently available, it is important to note the two major classes of heat recovery equipment - temperature boosting equipment and heat exchangers. The second law of thermodynamics states that for two bodies in thermal contact, energy will flow from the higher temperature body to the lower temperature body. Therefore, to transfer energy from lower temperature waste streams to higher temperature input streams, the temperature of the waste energy must be increased.

Table 6.5 shows the diagonal cells from Table 6.2. The source and sink temperature ranges are the same for these cells. For example, the source energy (waste heat) temperature range for the first cell in the diagonal is $80^{\circ} \mathrm{F}$ to $119^{\circ} \mathrm{F}$. That also is the temperature range of the sink energy (input energy).

Table 6.6 shows the set of cells where source/sink temperature combinations require temperature boosting technology, that is, where the source temperature is less than the sink temperature. An example of a temperature boosting technology is the electrically driven heat pump.

Table 6.7 shows the set of cells where source/sink temperature combinations can use heat exchanger heat recovery technology. In this set of source/ sink combinations, the source temperature is above the sink temperature. 
TABLE 6.5 Summary of 108 Source/Sink Matchups: Liquid and Gaseous Heat Streams Combined

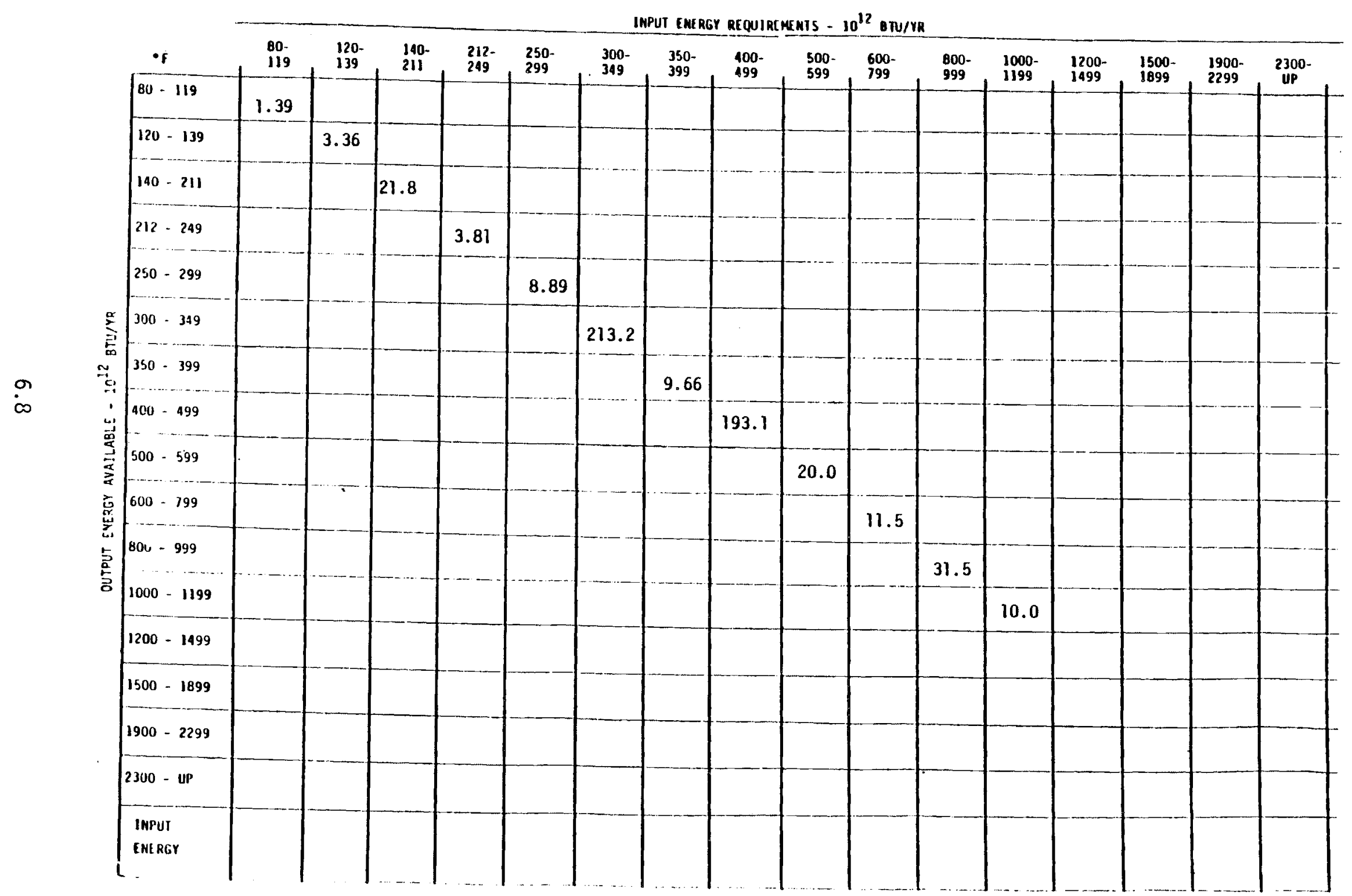


TABLE 6.6 Summary of 108 Source/Sink Matchups: Liquid and Gaseous Heat Streams Combined

\begin{tabular}{|c|c|c|c|c|c|c|c|c|c|c|c|c|c|c|c|c|c|}
\hline & & & & & & & & PUI ENERG & REQUIRE & HENIS - 1 & $0^{12} \quad 8 \pi / r$ & & & & & & \\
\hline & $-i$ & $\begin{array}{l}80- \\
119 \\
\end{array}$ & $\begin{array}{l}120- \\
139 \\
\end{array}$ & $\begin{array}{l}140- \\
211\end{array}$ & $\begin{array}{l}212- \\
249\end{array}$ & $\begin{array}{r}250- \\
299\end{array}$ & $\begin{array}{l}300- \\
349\end{array}$ & $\begin{array}{l}350- \\
399\end{array}$ & $\begin{array}{l}400- \\
499 \\
\end{array}$ & $\begin{array}{l}500- \\
599 \\
\end{array}$ & $\begin{array}{l}600- \\
799 \\
\end{array}$ & $\begin{array}{l}800- \\
999 \\
\end{array}$ & $\begin{array}{l}1000- \\
1199 \\
\end{array}$ & $\begin{array}{l}1200- \\
1499 \\
\end{array}$ & $\begin{array}{l}1500- \\
1899\end{array}$ & $\begin{array}{l}1900- \\
2299\end{array}$ & $\underset{\text { UP }}{2300-}$ \\
\hline & $80-119$ & & 2.46 & 35.6 & 42.0 & 517.5 & 265.3 & 150.7 & 553.4 & 21.0 & 61.8 & 81.1 & 10.9 & & 123.8 & & \\
\hline & $120-139$ & & & 14.1 & 24.1 & 27.7 & 46.7 & 13.3 & & & & 0.26 & 0.01 & & 0.26 & & \\
\hline & $140-211$ & & & & 18.2 & 246.5 & 195.3 & 79.6 & 86.1 & 1.51 & 7.48 & 67.2 & 10.1 & & 75.7 & & \\
\hline & $212-249$ & & & & & 449.4 & 246.3 & 48.1 & 5.24 & 1.69 & 0.30 & 42.7 & 1.60 & & 41.3 & & \\
\hline & $250-299$ & & & & & & 55.0 & 31.1 & 18.8 & & & 67.1 & & & 70.4 & & \\
\hline 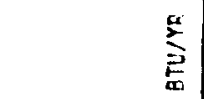 & $300-379$ & & & & & & & 19.4 & 12.8 & & & 14.8 & & & 14.8 & & \\
\hline 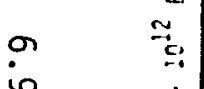 & $350-399$ & & & & & & & & 2.79 & & 12.7 & & & & & & \\
\hline $\begin{array}{l}\dot{u} \\
\ddot{\vec{a}} \\
\dot{a}\end{array}$ & $400-499$ & & & & & & & & & 1.85 & 24.2 & 32.6 & 10.1 & & 32.6 & & \\
\hline$\underset{3}{\vec{a}}$ & $500-599$ & & & & & & & & & & 2.34 & 69.4 & & & 77.8 & & \\
\hline 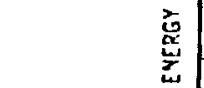 & $600-799$ & & & & & & & & & & & 67.1 & 3.14 & 0.01 & 120.3 & & \\
\hline $\begin{array}{l}5 \\
5 \\
5 \\
5\end{array}$ & $800-999$ & & & & & & & & & & & & 0.65 & 0.59 & 32.3 & & \\
\hline & $1000-1199$ & & & & & & & & & & & & & & 9.8 & & \\
\hline & $12 n 0-1499$ & & & & & & & & & & & & & & 123.8 & & \\
\hline & $1500-1899$ & & & & & & & & & & & & & & & & \\
\hline & $1900-2299$ & & & & & & & & & & & & & & & & \\
\hline & $2300-$ UP & & & & & & & & & & & & & & & & \\
\hline & $\begin{array}{l}\text { IMPUI } \\
\text { ENI RGY }\end{array}$ & & & & & & & & & & & & & & & & \\
\hline
\end{tabular}


TABLE 6.7 Summary of 108 Source/Sink Matchups: Liquid and Gaseous Heat Streams Combined

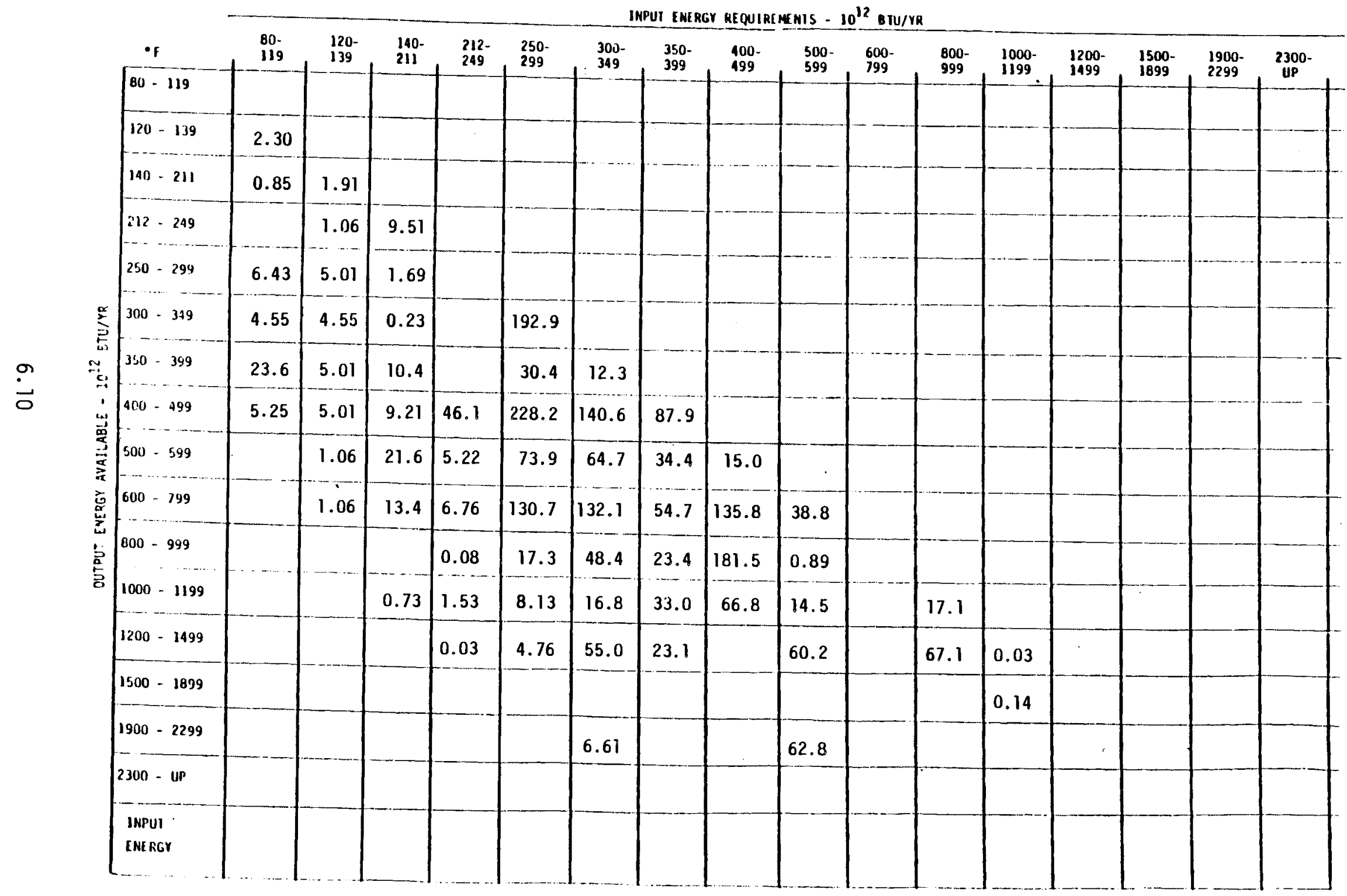




\subsection{CHARACTERIZATION OF HIGH-ENERGY SOURCE/SINK COMBINATIONS}

In this chapter the specific source/sink temperature combinations (cells) that have the greatest energy content in the summary matrix discussed in Chapter 6.0 are examined more closely. With these cells identified, the specific industry processes contributing to the highest energy cells are specified and the generic types of equipment involved identified.

Identifying the energy content of these source/sink combinations of energy streams can provide important insight to program planners as they evaluate the need and potential market for advanced waste heat recovery technology.

In the preceding section a summary matrix of source/sink values was developed for each of three classes of waste streams. In this section, the source/ sink temperature combinations that have the highest energy content are examined from these summary matrices. Table 7.1 identifies the cells that have the largest energy content for each class of heat stream. This table shows the source (output energy) and the sink (input energy) temperature, the cell's source/sink energy, and the cell's energy content ranked (from 1 to 5).

This assessment will now focus on selected high-energy source/sink combinations for each class of waste stream. Two criteria were used in selecting source/sink combinations to be evaluated. The first criterion was the energy content of source/sink temperature combinations. The second involved recognizing the fundamental difference between equipment that transfers heat from a higher temperature source to a lower temperature sink (heat exchangers) and equipment that thermodynamically boosts process stream temperatures (for example, heat pumps). These criteria suggest addressing two high-energy source/ sink combinations from each of the three classes of heat streams. The first source/sink combination to be examined has the source (output) temperature lower than the sink (input) temperature. Candidate source/sink combinations that meet these definitions are located above the diagonal running from the top left hand corner to the lower right hand corner of the summary matrix. The second source/sink combination examined has the source stream temperature above the sink temperature (below the diagonal). 
TABLE 7.1 Utility Summary Cells with the Highest Energy Values LIQUID AND GASEOUS HEAT STREAMS COMBINED

\begin{tabular}{lrll}
\hline $\begin{array}{l}\text { CELL } \\
\text { RANK }\end{array}$ & $\begin{array}{c}\text { OUTPUT ENERGY } \\
\text { TEMPERATURE RANGE }\end{array}$ & $\begin{array}{c}\text { INPUT ENERGY } \\
\text { TEMPERATURE RANGE }\end{array}$ & $\begin{array}{c}\text { UTILITY VALUE } \\
\left(10^{\star \star} 12 \mathrm{~B} \text { tu/Yr) }\right.\end{array}$ \\
\hline & $80-119 \mathrm{~F}$ & $400-499 \mathrm{~F}$ & 553.42 \\
2 & $80-119 \mathrm{~F}$ & $250-299 \mathrm{~F}$ & 524.39 \\
3 & $212-249 \mathrm{~F}$ & $250-299 \mathrm{~F}$ & 449.39 \\
4 & $80-119 \mathrm{~F}$ & $300-349 \mathrm{~F}$ & 265.26 \\
5 & $140-211 \mathrm{~F}$ & $250-299 \mathrm{~F}$ & 246.45 \\
\hline
\end{tabular}

LIQUID HEAT STREAMS ONLY

\begin{tabular}{lccc}
\hline $\begin{array}{l}\text { CELL } \\
\text { RANK }\end{array}$ & $\begin{array}{c}\text { OUTPUT ENERGY } \\
\text { TEMPERATURE RANGE }\end{array}$ & $\begin{array}{c}\text { INPUT ENERGY } \\
\text { TEMPERATURE RANGE }\end{array}$ & $\begin{array}{c}\text { UTILITY VALUE } \\
\left(10^{*} 12 \text { Btu/Yr) }\right.\end{array}$ \\
\hline 1 & $80-119 \mathrm{~F}$ & $140-211 \mathrm{~F}$ & 34.13 \\
2 & $80-119 \mathrm{~F}$ & $350-399 \mathrm{~F}$ & 23.28 \\
3 & $140-211 \mathrm{~F}$ & $140-211 \mathrm{~F}$ & 21.55 \\
4 & $120-139 \mathrm{~F}$ & $140-211 \mathrm{~F}$ & 14.07 \\
5 & $140-211 \mathrm{~F}$ & $350-399 \mathrm{~F}$ & 6.64 \\
\hline
\end{tabular}

GASEOUS HEAT STREAMS ONLY

\begin{tabular}{lccc}
\hline $\begin{array}{c}\text { CELL } \\
\text { RANK }\end{array}$ & $\begin{array}{c}\text { OUTPUT ENERGY } \\
\text { TEMPERATURE RANGE }\end{array}$ & $\begin{array}{c}\text { INPUT ENERGY } \\
\text { TEMPERATURE RANGE }\end{array}$ & $\begin{array}{c}\text { UTILITY VALUE } \\
\left(10^{* \star 12} \mathrm{~B} \text { tu/Yr) }\right.\end{array}$ \\
\hline & $212-249 \mathrm{~F}$ & $250-299 \mathrm{~F}$ & 496.61 \\
1 & $400-499 \mathrm{~F}$ & $250-299 \mathrm{~F}$ & 234.42 \\
3 & $300-349 \mathrm{~F}$ & $300-349 \mathrm{~F}$ & 214.72 \\
4 & $300-349 \mathrm{~F}$ & $250-299 \mathrm{~F}$ & 199.97 \\
5 & $400-499 \mathrm{~F}$ & $400-499 \mathrm{~F}$ & 192.91 \\
\hline
\end{tabular}




\section{COMBINED LIQUID AND GASEOUS HEAT STREAMS}

For combined liquid and gaseous heat streams (Table 6.2), the cell with the highest energy content above the diagonal has the source/sink temperature combination of $80-119^{\circ} \mathrm{F} / 400-499^{\circ} \mathrm{F}$ (553.4 trillion Btu/yr). Industry processes that contribute substantially to this cell's source/sink value are presented in Table 7.2. Table 7.2 shows the contribution that each industrial process makes to the total energy content of the cell (column 4). The industries are ranked in decreasing order of energy contribution. Cumulative contributions, as a percent of the total, are provided in column 5 . Petroleum Refining (2911-1) accounts for nearly $80 \%$ of the cel1's total energy content. The top two industries (Petroleum Refining and Nitrogenous Fertilizer) account for $96.59 \%$ of the total energy content. Eleven industries contribute to this cell of the summary matrix.

The types of process equipment that produce waste heat streams in the 80 - $119^{\circ} \mathrm{F}$ temperature range and process equipment that require input heat streams in the $400-499^{\circ} \mathrm{F}$ temperature range are listed in Table 7.3 for the top two industries (Petroleum Refining and for Nitrogenous Fertilizers). The

TABLE 7.2 Industry Processes Contributing to the $\left(80-119^{\circ} \mathrm{F}\right.$ Output Stream) $/\left(400-499^{0} \mathrm{~F}\right.$ Input Stream) Cell in the Combined Liquid and Gaseous Heat Stream Utility Matrix Summary

\begin{tabular}{|c|c|c|c|c|}
\hline $\begin{array}{l}\text { PROCESS } \\
\text { NUMBER }\end{array}$ & $\begin{array}{l}\text { INDUSTRY } \\
\text { PROCESS }\end{array}$ & $\begin{array}{c}\text { ENERGY } \\
\text { CONTIRUTION } \\
(10 \star \pi] 2 \text { Btu } / Y r) \\
\end{array}$ & $\begin{array}{l}\text { PER CEENT } \\
\text { OF TOTAL }\end{array}$ & $\begin{array}{l}\text { CUMAULATIVE } \\
\text { PER CENT }\end{array}$ \\
\hline $2911-1$ & PETROLEUM REFINING & 441.52 & 79.78 & 79.78 \\
\hline 2873-1 & NITROGENOUS FERTILIZER & 93.00 & 16.80 & 96.59 \\
\hline $2865-1$ & ETHYLBENZENE AND STYRENE & 4.49 & 0.81 & 97.40 \\
\hline $2821-3$ & PLASTICS, LDPE & 3.91 & 0.71 & 98.11 \\
\hline $2865-2$ & CUMENE AND PHENOL & 3.39 & 0.61 & 98.72 \\
\hline $2869-2$ & METHANOL & 2.60 & 0.47 & 99.19 \\
\hline \multirow[t]{2}{*}{$2821-5$} & PLASTICS, HDPE & 2.06 & 0.37 & 99.56 \\
\hline & OTHER (4) & $\frac{2.46}{553.4}$ & 0.44 & 100.00 \\
\hline
\end{tabular}


TABLE 7.3 Major Unit 8perations Supplying the Energy Sources and Sinks for the (80-119 $\mathrm{F}$ Output Stream)/(400-499 $\mathrm{F}$ Input Stream) Cell in the Combined Liquid and Gaseous Heat Stream Utility Matrix

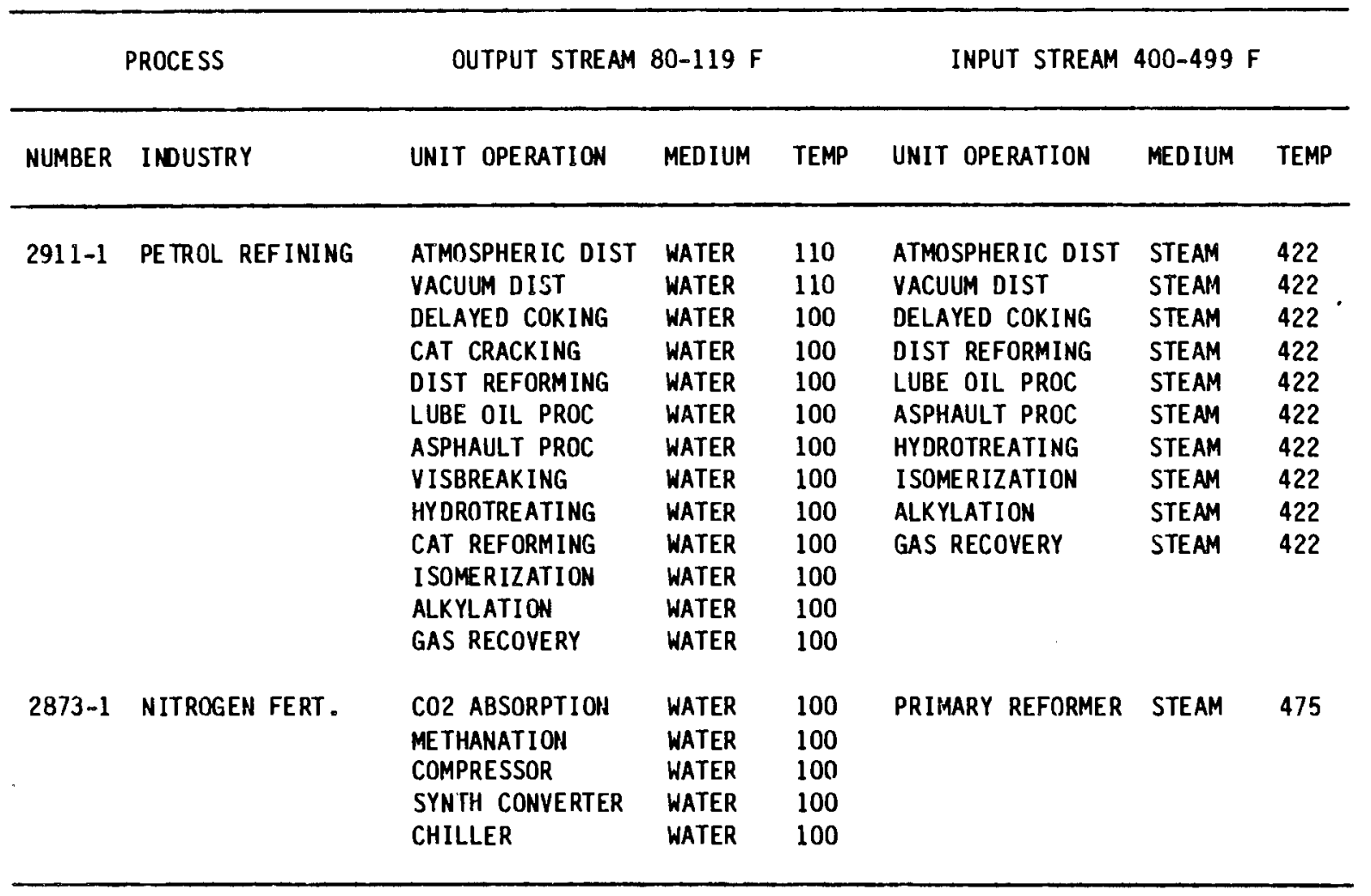

stream medium (in this case water or steam) and the stream temperature are also shown. Thirteen unit operations in Petroleum Refining produce hot waste water at 100 to $110^{\circ} \mathrm{F}$. Ten unit operations require steam at $422^{\circ} \mathrm{F}$.

Petroleum refineries typically have available large quantities of relatively low-cost by-product fuels. Justifying the expense of equipment to recover low-level waste heat may be more difficult in Petroleum Refining than in many other industries. For this reason, a second source/sink combination from the set of matrix cells above the diagonal is also examined. The source/ sink temperature combination $80-119^{\circ} \mathrm{F} / 250-299^{\circ} \mathrm{F}$ has an energy content of 517.5 trillion Btu/yr. The principal industries contributing to this total are listed in Table 7.4. Although two industry processes, Integrated Paper Mills (2621-2) and Integrated Paper Board Mills (2631-2), account for almost 
TABLE 7.4 Industry Processes Contributing to the $\left(80-119^{\circ} \mathrm{F}\right.$ Output Stream)/(250-299 $\mathrm{F}$ Input Stream) Cell in the Combined Liquid and Gaseous Heat Stream Utility Matrix Summary

\begin{tabular}{|c|c|c|c|c|}
\hline $\begin{array}{l}\text { PROCESS } \\
\text { NUMBER }\end{array}$ & $\begin{array}{l}\text { INDUSTRY } \\
\text { PROCESS }\end{array}$ & $\begin{array}{c}\text { ENERGY } \\
\text { CONTRIBUTION } \\
\left(10^{* * 12} \mathrm{Btu} / \mathrm{Yr}\right)\end{array}$ & $\begin{array}{l}\text { PER CENT } \\
\text { OF TOOTAL }\end{array}$ & $\begin{array}{l}\text { CUMULATI YE } \\
\text { PER CENT }\end{array}$ \\
\hline $2621-2$ & INTEGRATED PAPER MILLS & 166.40 & 32.16 & 32.16 \\
\hline $2631-2$ & INTEGRATED PAPER BOARD & 140.29 & 27.11 & 59.27 \\
\hline 2063-1 & BEET SUGAR REFINING & 25.66 & 4.96 & 64.23 \\
\hline $2874-1$ & PHOSPHATIC FERTILIZER & 22.08 & 4.27 & 68.49 \\
\hline $2611-1$ & KRAFT PULP MILLS & 20.40 & 3.94 & 72.43 \\
\hline $2046-1$ & WET CORN MILLING & 13.80 & 2.67 & 75.10 \\
\hline $2865-2$ & CUMENE AND PHENDL & 13.53 & 2.62 & 77.71 \\
\hline $2011-1$ & MEAT PACKING & 13.32 & 2.57 & 80.29 \\
\hline $2631-1$ & PAPER BOARD FINISHING & 10.15 & 1.96 & 82.25 \\
\hline $2062-1$ & CANE SUGAR REFINING & 9.59 & 1.85 & 84.10 \\
\hline $2869-2$ & METHANOL & 8.74 & 1.69 & 85.79 \\
\hline \multirow[t]{2}{*}{$2026-1$} & FLUID MILK & 7.88 & 1.52 & 87.31 \\
\hline & OTHER (39) & $\frac{65.66}{817.5}$ & 12.69 & 100.00 \\
\hline
\end{tabular}

$60 \%$ of the energy in this cel1, 51 industries have source/sink temperature combinations defined by this cell. The unit operations for the top 5 energy contributors to this cell are listed in Table 7.5. The source streams are typically water or water slurries at 82 to $95^{\circ} \mathrm{F}$ and the sink streams use steam at 250 to $292^{\circ} \mathrm{F}$.

The $400-499^{\circ} \mathrm{F} / 250-299^{\circ} \mathrm{F}$ cell from the liquid and gas stream summary has the highest energy content (228.2 trillion Btu/yr) of those cells with input stream temperatures less than the output stream temperature (below the diagonal from top left to bottom right). The major industry processes that contribute to this cell are listed in Table 7.6. Of the 28 industries that contribute to this source/sink combination, the top 5 account for $53.74 \%$ of the cell's total energy content. 
TABLE 7.5 Major Unit Operations Supplying the Energy Sources and Sinks for the $\left(80-119^{\circ} \mathrm{F}\right.$ Output Stream)/(250-299 $\mathrm{F}$ Input Stream) Cell in the Combined Liquid and Gaseous Heat Stream Utility Matrix Summary

\begin{tabular}{|c|c|c|c|c|c|c|c|}
\hline \multicolumn{2}{|r|}{ PROCESS } & \multicolumn{3}{|c|}{ OUTPUT STREAM 80-119 F } & \multicolumn{3}{|c|}{ INPUT STREAM 250-299 F } \\
\hline NUMBER & INDUSTRY & UNIT OPERATION & MEDIUM & TEMP & UNIT OPERATION & MED IUM & TFMP \\
\hline $2621-2$ & INTEG PAPER MILL & $\begin{array}{l}\text { MULT EVAP } \\
\text { BLEACHING } \\
\text { GENERATOR }\end{array}$ & $\begin{array}{l}\text { WATER } \\
\text { PLP \&WTR } \\
\text { WATER }\end{array}$ & $\begin{array}{l}95 \\
85 \\
95\end{array}$ & $\begin{array}{l}\text { MILT EVAP } \\
\text { BLEACHING } \\
\text { ORIER }\end{array}$ & $\begin{array}{l}\text { STEAM } \\
\text { STEAM } \\
\text { STEAM }\end{array}$ & $\begin{array}{l}292 \\
260 \\
250\end{array}$ \\
\hline $2631-2$ & INTEG PAPER BOARO & $\begin{array}{l}\text { MULT EVAP } \\
\text { HASHING } \\
\text { DECKER } \\
\text { REF INING }\end{array}$ & $\begin{array}{l}\text { WATER } \\
\text { PLP \&WTR } \\
\text { PLP\&WTR } \\
\text { PLP \&WTR }\end{array}$ & $\begin{array}{l}95 \\
90 \\
90 \\
95\end{array}$ & $\begin{array}{l}\text { MULT EVAP } \\
\text { DRIER }\end{array}$ & $\begin{array}{l}\text { STEAM } \\
\text { STEAM }\end{array}$ & $\begin{array}{l}292 \\
250\end{array}$ \\
\hline 2063-1 & BEET SUGAR & $\begin{array}{l}\text { VACUUM PANS } \\
\text { GENERATOR }\end{array}$ & $\begin{array}{l}\text { WATER } \\
\text { WATER }\end{array}$ & $\begin{array}{l}95 \\
95\end{array}$ & $\begin{array}{l}\text { DIFFUSION } \\
\text { JUICE IITR } \\
\text { HEATER } \\
\text { EVAPORATOR } \\
\text { GRAN. DRIER } \\
\text { VACUUM PANS }\end{array}$ & $\begin{array}{l}\text { STEAM } \\
\text { STEAM } \\
\text { STEAM } \\
\text { STEAM } \\
\text { STEAM } \\
\text { STEAM }\end{array}$ & $\begin{array}{l}250 \\
250 \\
250 \\
250 \\
250 \\
280\end{array}$ \\
\hline $2874-1$ & PHOS. FERTILIZER & $\begin{array}{l}\text { FILTER } \\
\text { EVAPORATOR } \\
\text { GENERATOR }\end{array}$ & $\begin{array}{l}\text { GYP } / W T R \\
\text { WATER } \\
\text { WATER }\end{array}$ & $\begin{array}{l}82 \\
92 \\
95\end{array}$ & EVAPORATOR & STEAM & 250 \\
\hline $2611-1$ & KRAFT PULP MILL & $\begin{array}{l}\text { MULT EVAP } \\
\text { BLEACHING } \\
\text { WASHING } \\
\text { DEWATER ING } \\
\text { GENERATOR }\end{array}$ & $\begin{array}{l}\text { WATER } \\
\text { PLP \&WTR } \\
\text { PLP \&WTR } \\
\text { WATER } \\
\text { WATER }\end{array}$ & $\begin{array}{l}95 \\
85 \\
90 \\
90 \\
95\end{array}$ & $\begin{array}{l}\text { MULT EVAP } \\
\text { RLEACHING } \\
\text { DRIER }\end{array}$ & $\begin{array}{l}\text { STEAM } \\
\text { STEAM } \\
\text { STEAM }\end{array}$ & $\begin{array}{l}292 \\
260 \\
260\end{array}$ \\
\hline
\end{tabular}

The major unit operations that produce waste heat streams in the 400 $499^{\circ} \mathrm{F}$ range and the unit operations that require heat streams in the 250 $299^{\circ} \mathrm{F}$ range are 1 isted in Table 7.7 . Here, the output streams are primarily stack gases and steam and the input streams are steam.

\section{GASEOUS WASTE HEAT STREAMS}

A similar analyses is provided using the summary matrix for gaseous only source/sink combinations. The $212-249^{\circ} \mathrm{F} / 250-299^{\circ} \mathrm{F}$ combination in the 
TABLE 7.6 Industry Processes Contributing to the $\left(400-499^{\circ} \mathrm{F}\right.$ Output Stream) $/\left(250-299^{\circ} \mathrm{F}\right.$ Input Stream) Cell in the Combined Liquid and Gaseous Heat Stream Utility Matrix

\begin{tabular}{|c|c|c|c|c|}
\hline $\begin{array}{l}\text { PROCESS } \\
\text { NUMBER }\end{array}$ & $\begin{array}{l}\text { INDUSTRY } \\
\text { PROCESS }\end{array}$ & $\begin{array}{c}\text { ENERGY } \\
\text { CONTRIBUTION } \\
\left(10^{\star \star \star 1} 12 \text { Btu }(Y r)\right.\end{array}$ & $\begin{array}{l}\text { PER CENT } \\
\text { OF TÓTAL }\end{array}$ & $\begin{array}{l}\text { CUMULATIVE } \\
\text { PER CENT }\end{array}$ \\
\hline $2421-1$ & SAW AND PLANING MILLS & 39.22 & 17.22 & 17.22 \\
\hline $2621-2$ & INTEGRATED PAPER MILLS & 27.61 & 12.10 & 29.32 \\
\hline $2631-2$ & INTEGRATED PAPER BOARD & 26.58 & 11.64 & 40.97 \\
\hline $2063-1$ & BEET SUGAR REF INING & 16.94 & 7.42 & 48.39 \\
\hline $2824-2$ & POLYPROPYLENE & 12.21 & 5.35 & 53.74 \\
\hline $2824-1$ & POLYESTER & 11.25 & 4.92 & $58.65^{\circ}$ \\
\hline $2221-1$ & PLASTICS, PVC & 10.66 & 4.67 & 63.33 \\
\hline $2631-1$ & PAPER BOARD FINISHING & 10.01 & 4.39 & 67.71 \\
\hline $2821-3$ & PLASTICS, LDPE & 8.81 & 3.86 & 71.57 \\
\hline $2621-1$ & PAPER MILLS, FINISHING & 8.01 & 3.51 & 75.08 \\
\hline $2026-1$ & FLUID MILK & 7.88 & 3.45 & 78.53 \\
\hline \multirow[t]{2}{*}{$2262-1$} & MAN-MADE FIBERS, FIN. & 7.52 & 3.30 & 81.83 \\
\hline & OTHER (16) & $\frac{46.50}{228.2}$ & 18.17 & 100.00 \\
\hline
\end{tabular}

gaseous only matrix has the highest energy content (496.6 trillion Btu/yr). The industrial processes contributing to this cell are listed in Table 7.8. Of the 21 industrial processes contributing to this cell, Integrated Paper Board Mills (2631-2) and Integrated Paper Mills (2621-2) together account for threequarters of the energy content. The unit operations for the 5 top energy contributing industries are listed in Table 7.9 with corresponding stream medium and stream temperature.

Similarly, the $400-499^{\circ} \mathrm{F} / 250-299^{\circ} \mathrm{F}$ source/sink combination in the gas only summary matrix has the highest energy content of cells with output stream temperature greater than input stream temperature $(234.4$ trillion Btu/ $y r)$. There are 35 industry processes in our data base with input and output streams having temperatures defined by this cell. The top 14 industrial 
TABLE 7.7 Major Unit Operations Supplying the Energy Sources and Sinks for the $\left(400-499^{\circ} \mathrm{F}\right.$ Output Stream $) /\left(250-299^{\circ} \mathrm{F}\right.$ Input Stream) Cel 1 in the Combined Liquid and Gaseous Heat Stream Utility Matrix Summary

\begin{tabular}{|c|c|c|c|c|c|c|c|}
\hline \multicolumn{2}{|r|}{ PROCESS } & \multicolumn{3}{|c|}{ OUTPUT STREAM 400-499 F } & \multicolumn{3}{|c|}{ INPUT STREAM 250-299 F } \\
\hline NUMBER & INDUSTRY & UNIT PROCESS & MED IUM & TEMP & UNIT PROCESS & MEDIUM & TEMP \\
\hline $2421-1$ & SAWMILLS & BOILER & STACK & 450 & DRIER KILN & STEAM & 250 \\
\hline \multirow[t]{3}{*}{$2621-2$} & INTEG PAPER MILL & EVAPORATOR & STEAM & 400 & MULT EVAP & STEAM & 292 \\
\hline & & BOILER & STACK & 450 & BLEACHING & STEAM & 260 \\
\hline & & & & & DR IER & STEAM & 250 \\
\hline \multirow{3}{*}{$2631-2$} & INTEG PAPER BOARD & EVAPORATOR & STEAM & 400 & MULT EVAP & STEAM & 292 \\
\hline & & KILN & STACK & 400 & DRIER & STEAM & 250 \\
\hline & & BOILER & STACK & 450 & & & \\
\hline \multirow[t]{6}{*}{$2063-1$} & BEET SUGAR & KILN DRIER & STACK & 400 & DIFFUSION & STEAM & 250 \\
\hline & & LIMING \& CARB. & $\mathrm{CO} 2$ & 400 & JUICE HTR & STEAM & 250 \\
\hline & & GENERATOR & STACK & 450 & HEATER & STEAM & 250 \\
\hline & & & & & EVAPORATOR & STEAM & 250 \\
\hline & & & & & GRAN DRIER & STEAM & 250 \\
\hline & & & & & VACUUM PANS & STEAM & 280 \\
\hline $2824-2$ & POLYPROPYLENE & BOILER & STACK & 400 & MELTING \& EXTR. & STEAM & 250 \\
\hline
\end{tabular}

processes contributing to this cell account for $85.37 \%$ of the total energy content. These industry processes are listed in Table 7.10. The relevant unit operations for the top five industrial processes in Table 7.10 are given in Table 7.11.

\section{LIQUID WASTE HEAT STREAMS}

For the liquid only summary matrix, the $80-119^{\circ} \mathrm{F} / 140-211^{\circ} \mathrm{F}$ source/ sink combination has the highest energy content (34.1 trillion Btu/yr). The 11 industrial processes contributing to this cell are listed in Table 7.12 and the relevant unit operations and stream characteristics for the top three industrial processes (accounting for $85 \%$ of the cell's energy content) are presented in Table 7.13 . 
TABLE 7.8 Industry Process Contributing to the $\left(212-249^{\circ} \mathrm{F}\right.$ Output Stream) $/\left(250-299^{\circ} \mathrm{F}\right.$ Input Stream) Cel1 in the Combined Liquid and Gaseous Heat Stream Utility Matrix Summary

\begin{tabular}{llccc}
\hline $\begin{array}{l}\text { PROCESS } \\
\text { NUMBER }\end{array}$ & $\begin{array}{l}\text { INDUSTRY } \\
\text { PROCESS }\end{array}$ & $\begin{array}{c}\text { ENERGY } \\
\text { CONTRIBUTION } \\
\left(10^{\star \star} 12 \text { BtU/Y }\right)\end{array}$ & $\begin{array}{c}\text { PER CENT } \\
\text { OF TOFAL }\end{array}$ & $\begin{array}{c}\text { CUMULATIVE } \\
\text { PER CENT }\end{array}$ \\
\hline $2631-2$ & INTEGRATED PAPER BOARD & 216.30 & 43.56 & 43.56 \\
$2621-2$ & INTEGRATED PAPER MILLS & 155.80 & 31.37 & 74.92 \\
$2631-1$ & FINISHING PAPER BOARD & 25.25 & 5.08 & 80.01 \\
$2621-1$ & FINISHING PAPER MILLS & 23.59 & 4.75 & 84.76 \\
$2661-2$ & INTEGRATED BLDG PAPER & 18.55 & 3.74 & 88.50 \\
$2611-1$ & KRAFT PAPER MILLS & 17.79 & 3.58 & 92.08 \\
$2075-1$ & SOYBEAN OIL MILLS & 11.01 & 2.22 & 94.30 \\
$2661-1$ & BLDG PAPER, FINISHING & 9.95 & 2.00 & 96.30 \\
$2011-1$ & MEAT PACKING PLANT & 5.06 & 1.02 & 97.32 \\
$2816-2$ & INORGANIC PIGMENTS & 4.56 & 0.92 & 98.24 \\
& OTHERS (11) & 8.74 & 1.76 & 100.00 \\
\hline
\end{tabular}

TABLE 7.9 Major Unit Operations Supplying the Energy Sources and Sinks for the $\left(212-249^{F}\right.$ Output Stream)/(250-299 $\mathrm{F}$ Input Stream) Ce11 in the Gas Waste Heat Stream Utility Matrix Summary

\begin{tabular}{|c|c|c|c|c|c|c|c|}
\hline & PROCESS & OUTPUT STREA & $212-249 \mathrm{~F}$ & & INPUT STREA & $250-299$ & \\
\hline NUMBER & I NDUS TRY & UNIT OPERATION & MEDIUM & TEMP & UNIT OPERATION & MEDIUM & TEMP \\
\hline $2631-2$ & INTEG. PAPER BOARD & $\begin{array}{l}\text { BLOW TANK } \\
\text { DRIER }\end{array}$ & $\begin{array}{l}\text { STEAM } \\
\text { EXHAUST }\end{array}$ & $\begin{array}{l}220 \\
240\end{array}$ & $\begin{array}{l}\text { MULT EVAP } \\
\text { DRIER }\end{array}$ & $\begin{array}{l}\text { STEAM } \\
\text { STEAM }\end{array}$ & $\begin{array}{l}292 \\
250\end{array}$ \\
\hline $2621-2$ & INTEG. PAPER MILLS & $\begin{array}{l}\text { BLOW TANK } \\
\text { DRIER }\end{array}$ & $\begin{array}{l}\text { STEAM } \\
\text { EXHAUST }\end{array}$ & $\begin{array}{l}220 \\
240\end{array}$ & $\begin{array}{l}\text { MULT EVAP } \\
\text { BLEACHING } \\
\text { DRIER }\end{array}$ & $\begin{array}{l}\text { STEAM } \\
\text { STEAM } \\
\text { STEAM }\end{array}$ & $\begin{array}{l}292 \\
260 \\
250\end{array}$ \\
\hline $2631-1$ & FIN. PAPER BOARD & DRIER & EXHAUST & 240 & DRIER & STEAM & 250 \\
\hline $2621-1$ & FIN. PAPER MILLS & DRIER & EXHAUST & 240 & DRIER & STEAM & 250 \\
\hline $2661-2$ & INTEG BLDG PAPER & DRIER & EXHAUST & 240 & DRIER & STEAM & 250 \\
\hline
\end{tabular}


TABLE 7.10 Industry Processes Contributing to the $\left(400-499^{\circ} \mathrm{F}\right.$ Output Stream)/(250-299 $\mathrm{F}$ Input Stream) Cell in the Combined Liquid and Gaseous Heat Stream Utility Matrix Summary

\begin{tabular}{llccc}
\hline $\begin{array}{l}\text { PROCESS } \\
\text { NUMBER }\end{array}$ & $\begin{array}{l}\text { INDUSTRY } \\
\text { PROCESS }\end{array}$ & $\begin{array}{c}\text { ENERGY } \\
\text { CONTRIBUTION } \\
(10 * 12 \text { BtU }\end{array}$ & $\begin{array}{c}\text { PER CEN }) \\
\text { OF TONAL }\end{array}$ & $\begin{array}{c}\text { CUMULATIVE } \\
\text { PER CENT }\end{array}$ \\
\hline $2421-1$ & SAW \& PLANING MILLS & 39.222 & 16.73 & 16.73 \\
$2621-2$ & INTEG. PAPER MILLS & 27.606 & 11.78 & 28.51 \\
$2631-2$ & INTEG. PAPERBOARD & 26.578 & 11.34 & 39.85 \\
$2063-1$ & BEET SUGAR REFINING & 16.942 & 7.23 & 47.07 \\
$2824-2$ & POLYPROPYLENE & 12.209 & 5.21 & 52.28 \\
$2824-1$ & POLYESTER & 11.245 & 4.80 & 57.08 \\
$2221-1$ & WEAVING MILLS & 10.661 & 4.55 & 61.63 \\
$2631-1$ & FINISHING PAPERBOARD & 10.011 & 4.27 & 65.90 \\
$2821-3$ & PLASTICS, LDPE & 8.808 & 3.76 & 69.65 \\
$2621-1$ & FINISHING PAPER MILLS & 8.010 & 3.42 & 73.07 \\
$2026-1$ & FLUID MILK & 7.878 & 3.36 & 76.43 \\
$2262-1$ & MAN-MADE FIBERS, FIN. & 7.521 & 3.21 & 79.64 \\
$2062-1$ & CANE SUGAR REF INING & 6.815 & 2.91 & 82.55 \\
$2661-2$ & BUILDING PAPER, INTEG. & 6.615 & 2.82 & 85.37 \\
& OTHER (21) & $\frac{34.285}{234.4}$ & 14.63 & 100.00 \\
\hline
\end{tabular}

The $140-211^{\circ} \mathrm{F} / 140-211^{\circ} \mathrm{F}$ cell of the liquid only summary matrix has the highest energy content ( 21.6 trillion Btu/yr) of those cells with output stream temperatures greater than or equal to input stream temperatures. The 8 industrial processes contributing to this cell are listed in Table 7.14. The relevant unit operations, stream mediums and stream temperatures are given for the top 3 industries in Table 7.15. 
TABLE 7.11 Major Unit Operations Supplying the Energy Sources and Sinks for the $\left(400-499^{\circ} \mathrm{F}\right.$ Output Stream $) /\left(250-299^{\circ} \mathrm{F}\right.$ Input Stream) Cell in the Gaseous Waste Heat Stream Utility Matrix Summary

\begin{tabular}{|c|c|c|c|c|c|c|c|}
\hline \multicolumn{2}{|r|}{ PROCESS } & \multicolumn{3}{|c|}{ OUTPUT STREAM $400-499 \mathrm{~F}$} & \multicolumn{3}{|c|}{ INPUT STREAM 250-299 F } \\
\hline NUMBER & INDUSTRY & UNIT OPERATION & MEDIUM & TEMP & UNIT OPERATION & MEDIUM & TEMP \\
\hline $2421-2$ & SAWMILLS & BOILER & STACK & 450 & DRYING KILN & STEAM & 250 \\
\hline $2621-2$ & INTEG PAPER MILLS & BOILER & STACK & 450 & $\begin{array}{l}\text { MULT EVAP } \\
\text { BLEACHING } \\
\text { DRIER }\end{array}$ & $\begin{array}{l}\text { STEAM } \\
\text { STEAM } \\
\text { STEAM }\end{array}$ & $\begin{array}{l}292 \\
250 \\
250\end{array}$ \\
\hline $2631-2$ & INTEG PAPER BOARD & BOILER & STACK & 450 & $\begin{array}{l}\text { MULT EVAP } \\
\text { DRIER }\end{array}$ & $\begin{array}{l}\text { STEAM } \\
\text { STEAM }\end{array}$ & $\begin{array}{l}292 \\
250\end{array}$ \\
\hline $2063-1$ & BEET SUGAR & $\begin{array}{l}\text { KILN DRIER } \\
\text { LIMING \& CARB. } \\
\text { GENERATOR }\end{array}$ & $\begin{array}{l}\text { STACK } \\
\text { CO2 } \\
\text { STACK }\end{array}$ & $\begin{array}{l}400 \\
400 \\
450\end{array}$ & $\begin{array}{l}\text { DIFFUSION } \\
\text { JUICE HTR } \\
\text { HEATER } \\
\text { EVAPORATOR } \\
\text { GRAN. DRIER } \\
\text { VACUUM PANS }\end{array}$ & $\begin{array}{l}\text { STEAM } \\
\text { STEAM } \\
\text { STEAM } \\
\text { STEAM } \\
\text { STEAM } \\
\text { STEAM }\end{array}$ & $\begin{array}{l}250 \\
250 \\
250 \\
250 \\
250 \\
280\end{array}$ \\
\hline $2824-2$ & POLYPROPYLENE & BOILER & STACK & 400 & MELTING \& EXTR & STEAM & 250 \\
\hline
\end{tabular}

TABLE 7.12 Industry Processes Contributing to the $\left(80-119^{\circ} \mathrm{F}\right.$ Output Stream)/(140-211 $\mathrm{F}$ Input Stream) Cell in the Liquid Heat Stream Utility Matrix Summary

\begin{tabular}{llccc}
\hline $\begin{array}{l}\text { PROCESS } \\
\text { NUMBER }\end{array}$ & $\begin{array}{l}\text { INDUSTRY } \\
\text { PROCESS }\end{array}$ & $\begin{array}{c}\text { ENERGY } \\
\text { CONTRIBUTION } \\
\left(10^{*} 12 \text { BtU/Yr }\right)\end{array}$ & $\begin{array}{c}\text { PER CENT } \\
\text { OF TOTAL }\end{array}$ & $\begin{array}{c}\text { CUMULATIVE } \\
\text { PER CENT }\end{array}$ \\
\hline $2082-1$ & MALT BEVERAGES & 11.617 & 34.04 & 34.04 \\
$2062-1$ & CANE SUGAR PROCESSING & 9.331 & 27.34 & 61.38 \\
$2011-1$ & MEAT PACKING PLANT & 8.093 & 23.71 & 85.09 \\
$2063-1$ & BEET SUGAR & 2.700 & 7.91 & 93.00 \\
$2033-2$ & CANNED VEGETABLES & 1.239 & 3.63 & 96.63 \\
$2033-1$ & CANNED FRUITS & 0.498 & 1.46 & 98.09 \\
$2033-3$ & CANNED DRINKS & 0.337 & 1.10 & 99.19 \\
$2822-2$ & BUTYL & 0.129 & 0.38 & 99.57 \\
$2823-2$ & POLYBUTADIENE & 0.115 & 0.34 & 99.91 \\
$3861-1$ & PHOTOGRAPHIC FILM & 0.019 & 0.06 & 99.97 \\
$2899-2$ & FATTY ACIDS & $\frac{0.010}{34.1}$ & 0.03 & 100.00 \\
\hline
\end{tabular}


TABLE 7.13 Major Unit Operations Supplying the Energy Sources and Sinks for the $\left(80-119^{\circ} \mathrm{F}\right.$ Output Stream)/(140-211 $1^{\circ} \mathrm{F}$ Input Stream) Cell in the Liquid Waste Heat Stream Utility Matrix Summary

\begin{tabular}{|c|c|c|c|c|c|c|c|}
\hline & PROCESS & OUTPUT STREAM & $80-140 \mathrm{~F}$ & & INPUT STREAM & $140-211$ & \\
\hline NUMBER & INDUSTRY & UNIT OPERATION & MEDIUM & TEMP & UNIT OPERATION & MEDIUM & TEMP \\
\hline \multirow[t]{3}{*}{$2082-1$} & MALT BEVERAGES & COMPRESSOR & $\begin{array}{l}\text { WATER } \\
\text { WATER }\end{array}$ & $\begin{array}{l}95 \\
95\end{array}$ & $\begin{array}{l}\text { COOKER } \\
\text { FILTER TUB }\end{array}$ & $\begin{array}{l}\text { WATER } \\
\text { WATER }\end{array}$ & $\begin{array}{l}180 \\
165\end{array}$ \\
\hline & & $\begin{array}{l}\text { GENERATOR } \\
\text { CHILLER }\end{array}$ & $\begin{array}{l}\text { WATER } \\
\text { WATER }\end{array}$ & 95 & $\begin{array}{l}\text { PASTEUR IZATION } \\
\text { PIER }\end{array}$ & $\begin{array}{l}\text { WAIER } \\
\text { WATER }\end{array}$ & $\begin{array}{l}165 \\
160\end{array}$ \\
\hline & & 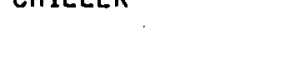 & & & CONTAINER WASH & WATER & 180 \\
\hline \multirow[t]{3}{*}{$2062-1$} & CANE SUGAR & $\begin{array}{l}\text { CHARCOAL WASHER } \\
\text { EVAPORTOR }\end{array}$ & $\begin{array}{l}\text { WATER } \\
\text { WATER }\end{array}$ & $\begin{array}{l}95 \\
95\end{array}$ & $\begin{array}{l}\text { MINGLER } \\
\text { CENTRIFUGE }\end{array}$ & WATER & 145 \\
\hline & & VACULM PANS & WATER & 95 & FILTER & WATER & 165 \\
\hline & & $\begin{array}{l}\text { LIQ SUGAR PROC. } \\
\text { GENERATOR }\end{array}$ & $\begin{array}{l}\text { WATER } \\
\text { WATER }\end{array}$ & $\begin{array}{l}95 \\
95\end{array}$ & MELTER & WATER & 195 \\
\hline \multirow[t]{2}{*}{$2011-1$} & MEAT PACKING & EVISCERATION & WATER & 120 & EVISCERATION & WATER & 140 \\
\hline & & VISCERA HANDLING & WATER & 120 & VISCERA HANDLING & WATER & 140 \\
\hline
\end{tabular}

TABLE 7.14 Industry Processes Contributing to the $\left(140-211^{\circ} \mathrm{F}\right.$ Output Stream)/(140-211 F Input Stream) Cell in the Liquid Heat Stream Utility Matrix Summary

\begin{tabular}{llccc}
\hline $\begin{array}{l}\text { PROCESS } \\
\text { NUMBER }\end{array}$ & $\begin{array}{l}\text { INDUSTRY } \\
\text { PROCESS }\end{array}$ & $\begin{array}{c}\text { ENERGY } \\
\text { CONTRIBUTION } \\
\left(10^{\star \star} 12 \text { BtU/Yr }\right)\end{array}$ & $\begin{array}{c}\text { PER CENT } \\
\text { OF TOTAL }\end{array}$ & $\begin{array}{c}\text { CUMULATIVE } \\
\text { PER CENT }\end{array}$ \\
\hline $2062-1$ & CANE SUGAR & 9.331 & 43.29 & 43.29 \\
$2082-1$ & MALT BEVERAGES & 7.484 & 34.72 & 78.01 \\
$2824-3$ & ACRYLICS & 2.328 & 10.80 & 88.81 \\
$2063-1$ & BEET SUGAR & 0.978 & 4.54 & 93.35 \\
$2823-1$ & RAYON & 0.596 & 2.77 & 96.12 \\
$2823-2$ & POLYBUTADIENE & 0.376 & 1.74 & 97.86 \\
$2822-2$ & BUTYL & 0.303 & 1.41 & 99.27 \\
$2033-2$ & CANNED VEGETABLES & $\frac{0.158}{21.6}$ & 0.73 & 100.00 \\
\hline
\end{tabular}


TABLE 7.15 Major Unit Operations Supplying the Energy Soruces and Sinks for the $\left(140-211^{F}\right.$ Output Stream $) /\left(140-211^{\circ}\right.$ Input Stream) Cell in the Liquid Waste Heat Stream Utility Matrix Summary

\begin{tabular}{|c|c|c|c|c|c|c|c|}
\hline & PROCESS & OUTPUT STREAM & $140-211 \mathrm{~F}$ & & INPUT STRE & $140-211$ & \\
\hline NUMBER & INDUSTRY & UNIT OPERATION & MED IUM & TEMP & UNIT OPERATION & MEDIUM & TEMP \\
\hline $2062-1$ & CANE SUGAR & $\begin{array}{l}\text { CENTRIFUGE } \\
\text { EVAPORATOR } \\
\text { VACUUM PANS } \\
\text { MIXER/CENTRIFUGE }\end{array}$ & $\begin{array}{l}\text { WATER } \\
\text { COND VAP } \\
\text { COND VAP } \\
\text { WATER }\end{array}$ & $\begin{array}{l}145 \\
170 \\
200 \\
140\end{array}$ & $\begin{array}{l}\text { MINGLER } \\
\text { CENTRIFUGE } \\
\text { FILTER } \\
\text { MELTER }\end{array}$ & $\begin{array}{l}\text { WATER } \\
\text { WATER } \\
\text { WATER } \\
\text { WATER }\end{array}$ & $\begin{array}{l}145 \\
145 \\
165 \\
195\end{array}$ \\
\hline $2082-1$ & MALT BEVERAGES & $\begin{array}{l}\text { CONTAINER WASH } \\
\text { PASTEURIZATION }\end{array}$ & $\begin{array}{l}\text { WATER } \\
\text { WATER }\end{array}$ & $\begin{array}{l}145 \\
150\end{array}$ & $\begin{array}{l}\text { COOKER } \\
\text { FILTER TUB } \\
\text { PASTEURIZATION } \\
\text { CONTAINER WASH }\end{array}$ & $\begin{array}{l}\text { WATER } \\
\text { WATER } \\
\text { WATER } \\
\text { WATER }\end{array}$ & $\begin{array}{l}180 \\
165 \\
160 \\
180\end{array}$ \\
\hline $2824-3$ & ACRYLICS & $\begin{array}{l}\text { HEATED CHAMBER } \\
\text { GENERATOR }\end{array}$ & $\begin{array}{l}\text { WATER } \\
\text { WATER }\end{array}$ & $\begin{array}{l}160 \\
140\end{array}$ & HEATED CHAMBER & WATER & 180 \\
\hline
\end{tabular}




\section{REFERENCES}

Drexel University. 1980. Energy Analysis of 108 Industrial Processes. Prepared by Drexel Univesity for the U.S. Department of Energy, Washington, D.C.

General Electric. 1981. Industrial Process Heat Pump Service Study. DOE/CS/40449-1, Prepared by General Electric for the U.S. Department of Energy, Washington, D.C.

Hame 1, B., et al. 1979. Energy Analysis of 108 Industrial Processes, Phase I of Industrial Applications Study. Prepared for the U.S. Department of Energy by Drexel University, Philadelphia, Pennsylvania.

Hedman, B. and H. Brown. 1980. Application of the Second Law of Thermodynamics to Industrial Processes. Drexel University, Philadelphia, Pennsylvnia.

Latour, S. R. and J. G. Menningman. 1981. Waste Heat Recovery Potential in Selected Industries. DSS Engineers, Inc., Fort Lauderdale, Florida.

Nydick, S. E., et al. June 1976. A Study of Inplant Electric Power Generation in the Chemical, Petrol eum Refining, and Paper and Pulp Industries. FEA/D76/321. Thermo Electron Corporation, Wal tham, Massachusetts.

Palmer, W. B., H. E. Gerlaugh, and R. R. Priestley. April 1980. Cogeneration Technology Alternatives Study (CTAS), General Electric Company Final Report, Volume III - Industrial Processes. DOE/NASA-0031-80/3. General Electric Company, Schenectady, New York.

Reding, J. T. et al. 1975. Energy Consumption: The Chemical Industry. EPA 650/2-75-032a, PB 241927 , Environmental Protection Agency, washington, D.C.

Solar Energy Research Institute (SERI) and Synergic Resources Corporation (SRC). 1980. Current and Future Industry Energy Services Characterizations, Vol. I. Available from Solar Energy Research Institute, Golden, Colorado.

Synergic Resources Corporation. 1982. Review of Industrial Energy Data Bases. Prepared by Synergic Resources Corporation for Electric Power Research Institute, Palo Alto, California.

U.S. Department of Commerce. 1972. Standard Industrial Classification Manual. Office of Federal Statistical Policy and Standards, U.S. Department of Commerce, Washington, D.C.

U.S. Department of Commerce. 1981. 1977 Census of Manufacturers Fuels and Electric Energy Consumed. U.S. Department of Commerce, Bureau of Census, Washington, D.C. 
U.S. Department of Commerce. 1982. 1980 Annual Survey of Manufactures, Fuels and Electric Energy Consumed, Industry Groups and Industries. U.S.

Department of Commerce, Bureau of Census, Washington, D.C.

U.S. Department of Energy. 1983. Energy Conservation Technology R\&D Program

Plan. U.S. Department of Energy, Washington, D.C.

United Technologies Corporation. January 1980. Cogeneration Technology Alternatives Study (CTAS), United Technologies Corporation Final Report, Volume II Industrial Process Characteristics. D0E/NASA/0030-80/2. South Windsor, Connecticut. 
APPENDICES A - F 


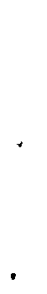




\section{APPENDICES}

Appendices $A$ through $F$ describe the manufacturing processes, heat-transfer operations, and waste heat characteristics of 35 processes in 6 industrial categories: 1) chemicals, 2) petroleum, 3) primary metals, 4) pulp and paper, 5) stone, $c l$ ay and $g l a s s$, and 6) textiles. These 6 industries account for almost $80 \%$ of the total energy used by the manufacturing sector.

This information was gathered through an extensive survey of the literature. In general, qualitative process descriptions are readily available in the literature. Much less information is reported on the location and characteristics of sources of waste heat. Almost no information on the quantity of waste heat by process is presented in this literature. 
APPENDIX A

CHEMICALS - SIC 28 


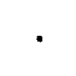

.

. 
APPENDIX A

CHEMICALS - SIC 28

The chemicals industry encompasses the manufacture of thousands of organic and inorganic chemicals by a variety of processes. Four product groups consume more than $90 \%$ of the total energy used by the chemicals industry: 1) industrial inorganics, 2) plastics and synthetics, 3) industrial organics, and 4) agricultural chemicals. The chemicals industry is the largest user of energy in the manufacturing sector, accounting for $24.5 \%$ of total energy use.

This section provides a discussion of the manufacturing processes, heattransfer operations, and waste heat streams for the industries listed below:

SIC

2812

Alkalies and Chlorine

- chlorine

- caustic soda

- soda ash

2813

Industrial Gases

- oxygen

- nitrogen

2819

Other Industrial Inorganics

- sulfuric acid

- elemental phosphorus

- phosphoric acid

2821

Plastics Materials and Resins

- low-density polyethylene

- high-density polyethylene

- polyvinyl chloride

- polystyrene

2824

Organic Fibers

- polyester

- nylon

- acrylic

2865

Cyclic Crudes and Intermediates

- styrene

- phenol

2869

Aliphatic Organic Chemicals

- ethylene

2873

Nitrogenous Fertilizers

- ammonia 
ALKALIES AND CHLORINE - SIC 2812

The chemicals in this classification include chlorine, caustic soda $(\mathrm{NaOH})$, caustic potash $(\mathrm{KOH})$, soda ash $\left(\mathrm{Na}_{2} \mathrm{CO}_{3}\right)$, and other miscellaneous alkalies. Chlorine and caustic soda account for most of the production and most of the energy use. Soda ash ranks third in production volume and energy use.

Process Descriptions

Alkalies and chlorine are manufactured from common salt and trona ore $\left(\mathrm{Na}_{2} \mathrm{CO}_{3} \cdot \mathrm{NaHCO}_{3}\right)$. Chlor-alkali plants range in capacity from about 2000 tons/year for plants captive to the paper industry to over two million tons/ year. The typical size of a commercial chlor-alkali plant is about 150,000 tons/year (UTC 1980). The five plants that produce natural soda ash range in capacity from 1000 to 2500 thousand short tons per year (Bumines 1980). The manufacturing steps used to produce chlorine, caustic soda and soda ash are described below.

\section{Chlorine and Caustic Soda}

Chlorine and caustic soda are primarily manufactured by the electrolysis of a salt brine solution. Most chlorine and caustic soda is produced using diaphram cells as shown in Figure A.1. Mercury cells, which accounted for about $25 \%$ of the production prior to 1976, are being phased out due to environmental problems (Latour 1981).

The salt in underground deposits is mined by pumping water into the cavity and forcing saturated brine to the surface. Steam is used to preheat the brine, and soda ash and caustic soda are added to precipitate calcium and magnesium, respectively. The purified, filtered brine is further heated to 175 to $200^{\circ} \mathrm{F}$ by direct steam injection.

In the cell, the application of a direct current dissociates salt and water according to the following reaction:

$$
2 \mathrm{Na}^{+}+2 \mathrm{Cl}^{-}+2 \mathrm{H}_{2} \mathrm{O} \longrightarrow \underset{\text { (anode) }}{2} \underset{\text { (cathöde) }}{+\mathrm{Cl}_{2}+\mathrm{H}_{2}^{\dagger}}
$$




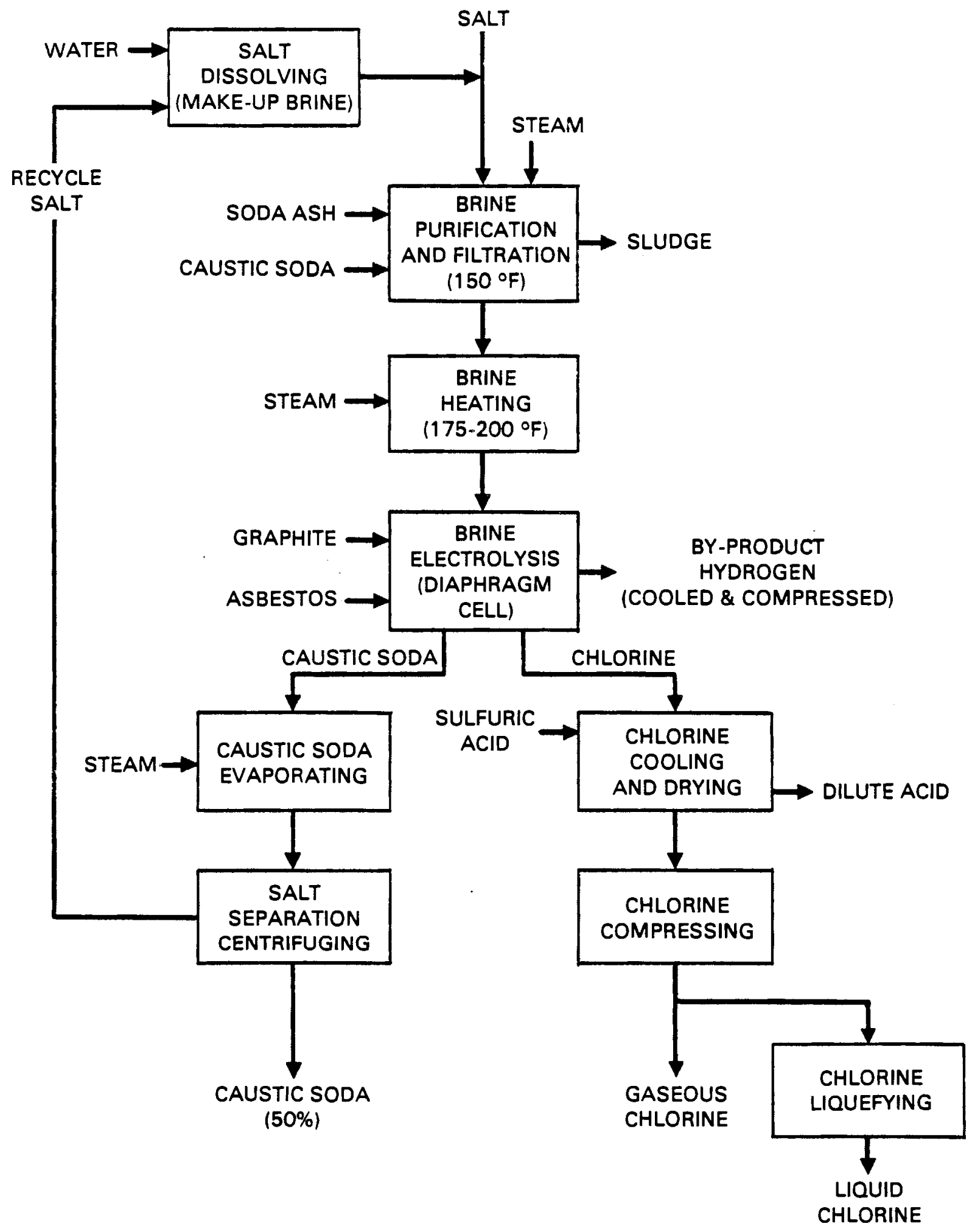

FIGURE A.1. Chlorine and Caustic Soda Flow Diagram (Adapted from Sittig 1977) 
Chlorine gas is drawn from the top of the cells through a heat exchanger and a drying tower. Chlorine flows countercurrent to strong sulfuric acid in the drying tower, which strips the water from the chlorine product. The chlorine may then be compressed for storage as a gas at 30 to 100 psig or liquified.

By-product hydrogen is drawn off at the cathode, cooled and compressed. The dilute cell liquor overflows to the caustic evaporation process. The cell liquor is concentrated in multiple-effect evaporators from a concentration of about 10 to $50 \%$. Most of the caustic is sold in this form. Some caustic is further concentrated to a 70 to $75 \%$ solution in a single-effect evaporator or to anhydrous caustic in evaporators using molten salt or oils as a heattransfer medium.

\section{Soda Ash}

Soda ash is manufactured by two common processes: the Solvay (or synthetic process) and the Trona process. Because of the abundance of natural soda ash in the United States, the Trona process is the most common. Because few Solvay plants are in operation, only the Trona process will be discussed.

Figure A.2 shows the major steps in the Trona process. Crushed trona is calcined in a rotary kiln at 320 to $390^{\circ} \mathrm{F}$ to drive off carbon dioxide and water as follows:

$$
2 \mathrm{Na}_{2} \mathrm{CO}_{3} \cdot \mathrm{NaHCO}_{3} \longrightarrow 3 \mathrm{Na}_{2} \mathrm{CO}_{3}+5 \mathrm{H}_{2} \mathrm{O}^{\dagger}+\mathrm{CO}_{2}^{\uparrow}
$$

The calcined ore is then dissolved in water, insoluables are removed, and the solution is filtered and treated with activated carbon to remove impurities. The resulting liquid is concentrated in triple-effect evaporators and sodium carbonate monohydrate $\left(\mathrm{Na}_{2} \mathrm{CO}_{3} \cdot \mathrm{H}_{2} \mathrm{O}\right)$ precipitates. The crystals are separated by centrifugation and calcined to remove the water of crystallization. The product is then cooled and screened prior to shipping.

Natural gas is the principal fuel for calcining soda ash. Most natural soda ash facilities have the multiple fuel capabilities of natural gas, fuel oil, and coal (BuMines 1980). 


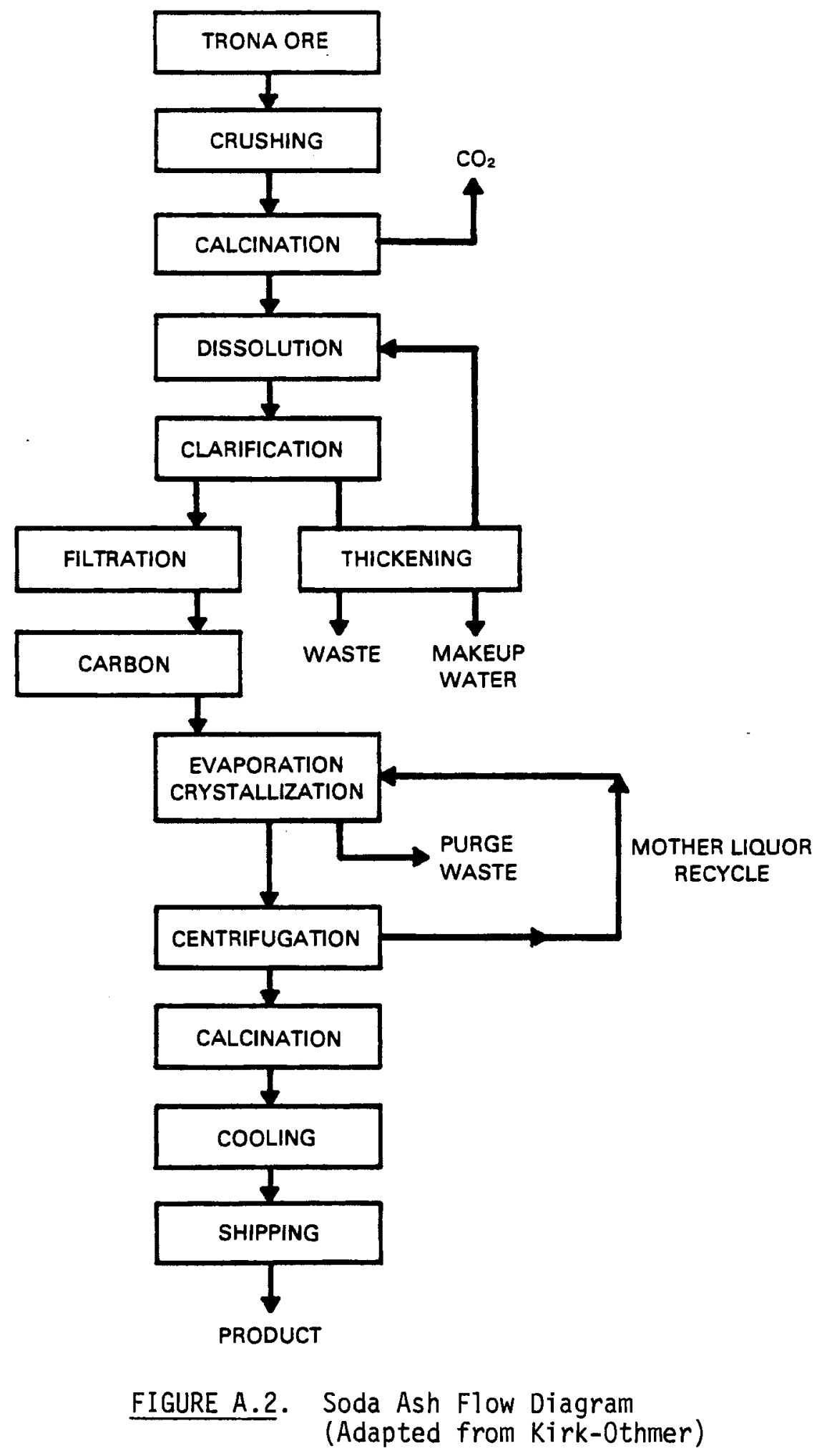

A. 5 
Heat-Transfer Operations

The major heat-transfer operations in chlorine/caustic and soda ash production are listed in Table A.1. Non-contact operations are those in which the heat-transfer fluid and the medium being heated or cooled are kept separate by a heat-transfer surface. In direct-contact operations, the heat-transfer fluid and the media being heated or cooled come into contact.

Steam is generated by burning fossil fuels, primarily natural gas and coal. It is expected that the industry will shift increasingly to the use of coal as the availability of natural gas diminishes and the price increases. About $80 \%$ of the steam is used for caustic evaporation in a triple-effect evaporator. About 7500 lbs/electrochemical unit ${ }^{(a)}$ is used in the evaporators at about $90 \mathrm{psi}$ (UTC 1980). The first two stages operate at about $140^{\circ} \mathrm{F}$

(a) Electrochemical unit (E.C.U.) is one ton of chlorine plus 1.12 tons of caustic in a $50 \%$ solution.

TABLE A.1. Heat-Transfer Operations in Chlorine/ Caustic and Soda Ash Production

\section{CHLORINE/CAUSTIC}

- Steam Generation

o Brine Heating

- Caustic Evaporating

o Chlorine Cooling

- Hydrogen Cooling

SODA ASH

o Trona Calcining

- Evaporation

- Monohydrate Calcining

o Product Cooling
Type of Heat Transfer

non contact

direct contact

non contact

direct contact

direct contact

direct contact

non contact

non contact

di rect contact
Heat-Transfer Medium

combustion gases

steam

steam

water

water

combustion gases

steam

steam

air 
and $200^{\circ} \mathrm{F}$. The third stage generally operates above $300^{\circ} \mathrm{F}$. Typically, all of the process steam is introduced in the third stage and the steam evaporated from the caustic soda solution provides the process heat for the first two stages. About 1900 lbs/E.C.U. of low-pressure steam is used for brine heating by direct injection.

Chlorine and hydrogen are cooled by direct contact with water. Natural gas is the principal fuel for calcining trona, although most plants now have multiple fuel capability. Calcination takes place in rotary kilns or vertical kilns. Steam is often used to dehydrate the monohydrate in rotary dryers. The dryers utilize finned tubes to enhance heat transfer. The product is air cooled.

Waste Heat Streams

The major sources of waste heat in chlorine and caustic production are listed in Table A.2. The largest source of waste heat is the water vapor evaporated from the cell liquor in the triple-effect evaporators. About 4700 pounds of water vapor are discharged from the evaporators per ton of chlorine produced at about 1.7 psia and $40^{\circ} \mathrm{F}$ above ambient (Latour 1981).

TABLE A.2. Waste Heat Rejected from Chlorine and Caustic Production (Sittig 1977)

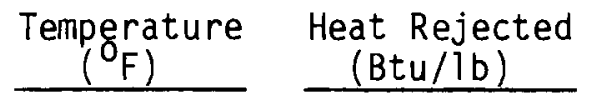

Diaphram Cell:

- Radiation and Convection

- $\mathrm{H}_{2}$ By-Product Cooling

- Chlorine Cooling

190

190

$1100(a)$
20

Caustic Evaporators:

- Radiation and Convection

- Water Vapor

- Caustic Cooling
(a) Heat rejected per pound of chlorine
(b) Heat rejected per pound of caustic soda as $100 \%$ caustic soda 
Cooling water is used to cool process liquids and vapors. Much of this cooling occurs by direct contact with the process stream. Thus, the discharged cooling waters may be contaminated. Table A.3 lists the cooling water sources, temperatures, flow rates, heat content, and composition for a representative $1.5 \mathrm{million}$ pound/day chlorine plant.

- The major energy users in the production of soda ash is the calcination of raw trona, evaporation, and product drying. Table A.4 provides a heat balance for the ore kiln and the rotary monohydrate dryer. Table A.5 identifies the waste heat sources, temperatures, and quantities for the trona process. The only liquid waste stream is the condensate from the evaporator. This stream rejects about 156,000 Btu per ton of soda ash (Latour 1981).

\section{INDUSTRIAL GASES - SIC 2813}

The production of oxygen and nitrogen accounts for almost $70 \%$ of the production of industrial gases and uses almost $60 \%$ of the total energy consumed by the industrial gas industry. The production methods, heat-transfer operations, and waste heat streams associated with these chemicals are discussed below.

\section{Process Description}

Oxygen and nitrogen are manufactured by the cryogenic air separation process shown in Figure A.3. High-pressure cycles that were commonly used in the 1950s are becoming obsolete and are being replaced by the slightly less energy-efficient and less costly low-pressure plants. The following descriptions will refer to the low-pressure cycle. Equipment required for air separation is classified as that which operates at room temperature and that which operates below room temperature, as shown in Table A.6.

The air is first filtered to remove impurities. Then the air is compressed to approximately 75-85 psi in a centrifugal compressor and is aftercooled. Any liquid water is extracted before the air is sent to a reversing heat exchanger where it is cooled almost to its dew-point temperature. As the air is cooled, first moisture and then carbon dioxide condenses and freezes on 
TABLE A.3 Sample Chlorine/Caustic Plant Waste Heat Stream Summary (Schmidt 1978)

\begin{tabular}{|c|c|c|c|c|c|c|c|}
\hline Source & $\begin{array}{l}\text { Throughput } \\
\text { (1b/day) }\end{array}$ & $\begin{array}{c}\text { Flow } \\
\left(10^{3} \mathrm{lb} / \mathrm{hr}\right)\end{array}$ & $\begin{array}{l}\text { Temperature } \\
\text { Input }\left({ }^{\circ} \mathrm{F}\right)\end{array}$ & Composition & $\begin{array}{c}\text { Phase }{ }^{\star} \\
\text { Disposition }\end{array}$ & $\begin{array}{l}\text { Heat Content } \\
\left(10^{7} \text { Btu/Day }\right)\end{array}$ & $\begin{array}{c}\text { Specific } \\
\text { Energy } \\
\text { (Btu/lb) }\end{array}$ \\
\hline Chlorine/Caustic Plant & $1,534,246$ & & & & & 426.8 & 2,782 \\
\hline - Brine heat exchanger & & 1,152 & $180 / 122$ & $25 \%$ salt & $\mathrm{L} / \mathrm{CW}$ & $(262.1)$ & \\
\hline - Primary hydrogen coolers & & 3 & $230 / 105$ & Moist $\mathrm{H}_{2}$ & $\mathrm{~V} / \mathrm{CH}$ & $(10.6)$ & \\
\hline - Hydrogen compressors & & 1 & & Moist $\mathrm{H}_{2}$ & $\mathrm{~V} / \mathrm{CW}$ & $(4.3)$ & \\
\hline - Primary chlorine coolers & & 47 & $175 / 100$ & wet $\mathrm{Cl}_{2}$ & $\mathrm{~V} / \mathrm{CW}$ & $(15.6)$ & \\
\hline - Chlorine compressor oil coolers & & & $150 /$ & oil & $\mathrm{L} / \mathrm{CW}$ & $(1.7)$ & \\
\hline - Dechlorinating flash tank & & 2 & $180 / 125$ & $\mathrm{Cl}_{2}, \mathrm{air}, \mathrm{H}_{2} \mathrm{O}$ & $\mathrm{V} / \mathrm{CW}$ & $(11.5)$ & \\
\hline - Chlorine liquefaction condensers & & & $105 / 100$ & & $\mathrm{~L}-\mathrm{V} / \mathrm{CW}$ & $(41.4)$ & \\
\hline - Chlorine liquid freon compressors & & & & & CW & $(1.5)$ & \\
\hline - Caustic coolers & & 90 & $230 / 110$ & $50 \%$ caustic & $\mathrm{L} / \mathrm{CW}$ & $(19.9)$ & \\
\hline - Sodium hypochlorite cooler & & 40 & $150 / 140$ & & $\mathrm{~L} / \mathrm{CW}$ & $(5.1)$ & \\
\hline - Compressant intercooler & & 2 & $220 / 110$ & $\mathrm{Cl}_{2}$ & $\mathrm{~V} / \mathrm{CW}$ & $(6.0)$ & \\
\hline - Di amond freon compressors & & 29 & $235 / 105$ & MW 865 & CW & $(1.2)$ & \\
\hline - Di amond snift compressors & & 16 & $250 / 230$ & dry $\mathrm{Cl}_{2}$ & $\mathrm{v} / \mathrm{CW}$ & $(1.2)$ & \\
\hline - Di amond freon condensers & & & $230 / 105$ & & $\mathrm{~L}-\mathrm{V} / \mathrm{CW}$ & $(12.0)$ & \\
\hline - Water chillers & & 960 & & $\mathrm{H}_{2} \mathrm{O}$ & $\mathrm{L} / \mathrm{CW}$ & $(33.31)$ & \\
\hline
\end{tabular}

$\star_{L}=$ Liquid, $V=$ Vapor, $C W=$ Cooling Water. 
TABLE A.4. Heat Balance for Kiln and Rotary Dryer (Sittig 1977)

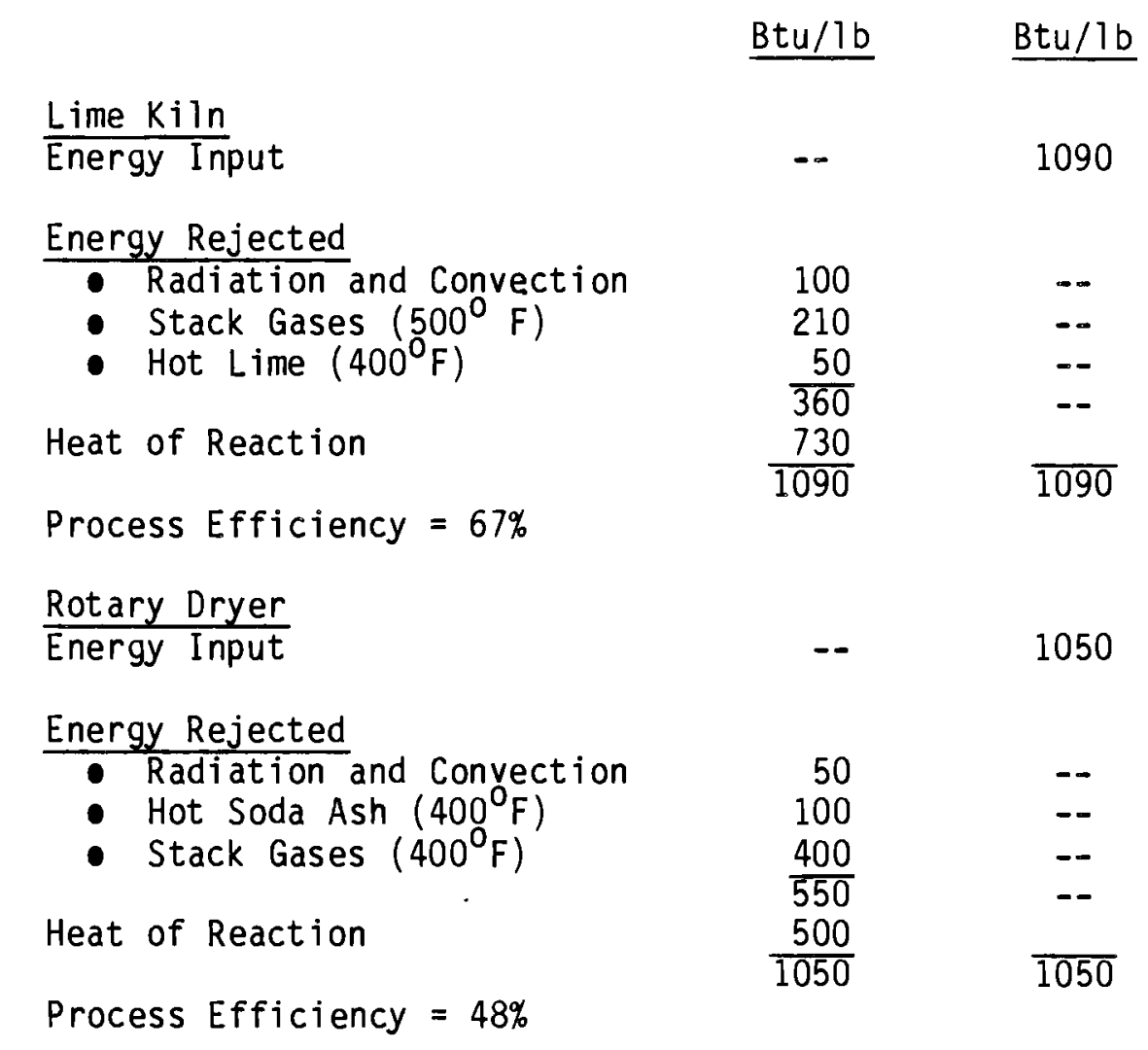

TABLE A.5. Sources, Quantities and Temperatures of Waste Heat (Latour 1981)

\begin{tabular}{|c|c|c|c|}
\hline Source & Type & $\begin{array}{c}\text { Temperature } \\
\left(\mathrm{O}_{\mathrm{F}}\right)\end{array}$ & $\mathrm{Ft}^{3} / \mathrm{Ton}$ \\
\hline Trona Calcination & Flue Gas & 405 & 150,000 \\
\hline Boiler & Stack Gas & 370 & 155,000 \\
\hline Monohydrate Drying & Air \& Water Vapor & 185 & 35,900 \\
\hline Soda Ash Cooling & Radiant Heat & 300 & - \\
\hline
\end{tabular}




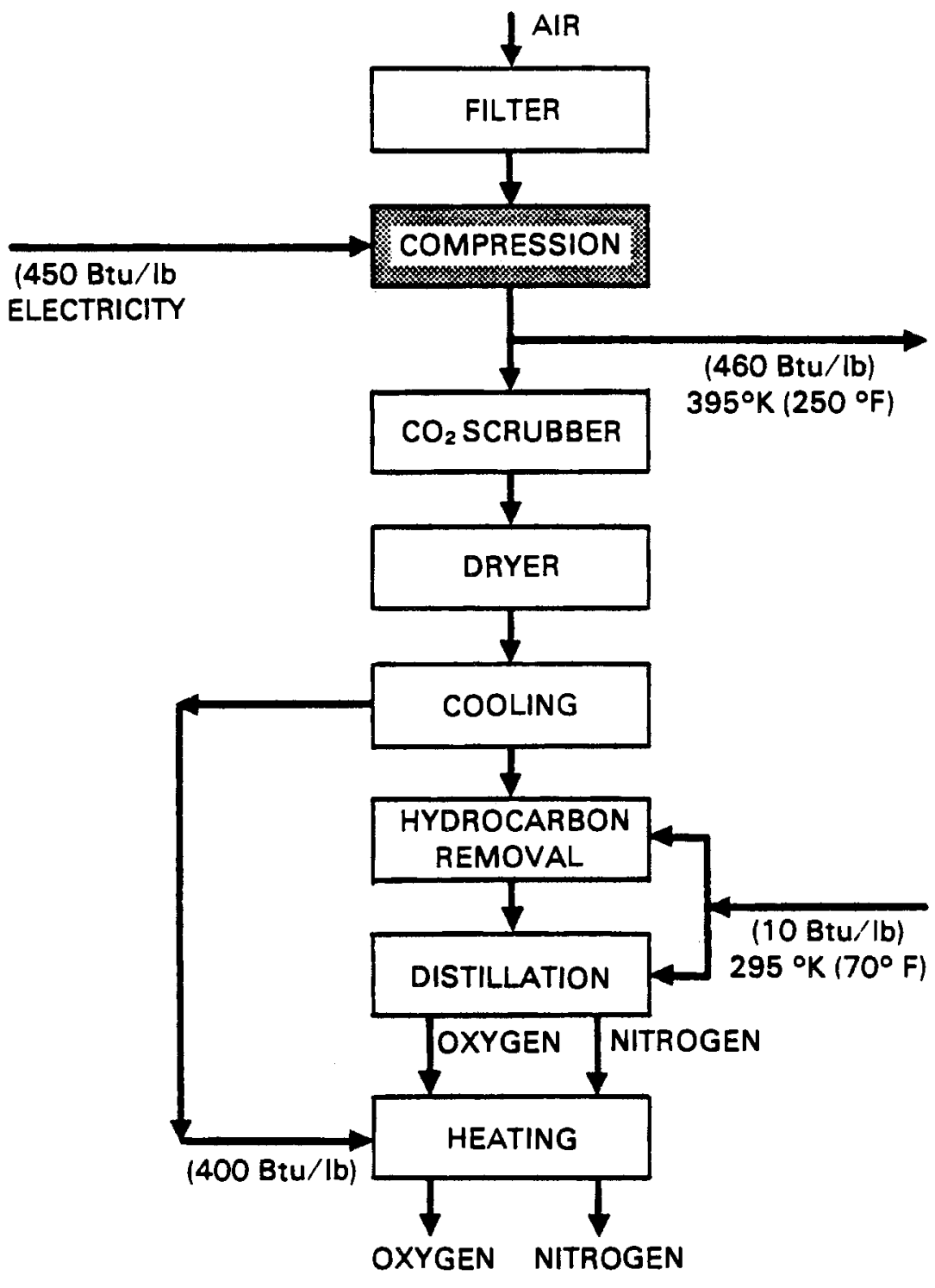

FIGURE A.3 0xygen and Nitrogen Production (Sittig 1977)

the walls of the heat exchanger. The air exits the reversing heat exchanger completely dry and with $99 \%$ of the carbon dioxide removed (Shen 1980). Fixedbed absorption is used to remove any remaining carbon dioxide and any hydrocarbons from the air stream. 
TABLE A.6. Classification of Air Separation Equipment

(Shen 1980)

Room Temperature:

Below Room Temperature:

\author{
Compressors \\ Inter-Coolers \\ After-Coolers \\ $0 i 1$ Separators \\ Water Separators \\ Heat Exchangers \\ Cleanup Equipment \\ Expanders \\ Distillation Columns
}

The air is then fed to the bottom tray of a double-column rectifier. The double-column rectifier is composed of two tray distillation columns that are connected by a heat exchanger as shown in Figure A.4. The heat exchanger is the condenser for the lower column and the reboiler for the upper column. Nitrogen is more volatile than oxygen; thus it ascends the column and oxygen descends. The nitrogen stream is divided as it leaves the condenser/reboiler. A portion of the stream returns to the lower column as reflux and the other is routed to the upper column, through the nitrogen superheater, to serve as reflux in the upper column. The liquefied oxygen stream exiting the lower column is cooled in the nitrogen superheater and becomes the feed for the upper column. The oxygen product is drawn off as a saturated vapor from the top of the upper column. The low-purity waste stream is removed several trays from the top of the column.

Compression is the most energy-intensive step in the production of these industrial gases. Compressors are operated with electricity. Table A.7 summarizes the total energy requirements for producing oxygen and nitrogen.

0xygen plants range in capacity from 10 tons/day up to 6000 tons/day. The larger plants contain several modules since the technical limit on the capacity of a single module is 2000 tons/day. The average capacity is about 780 tons/day (Palmer 1980). 


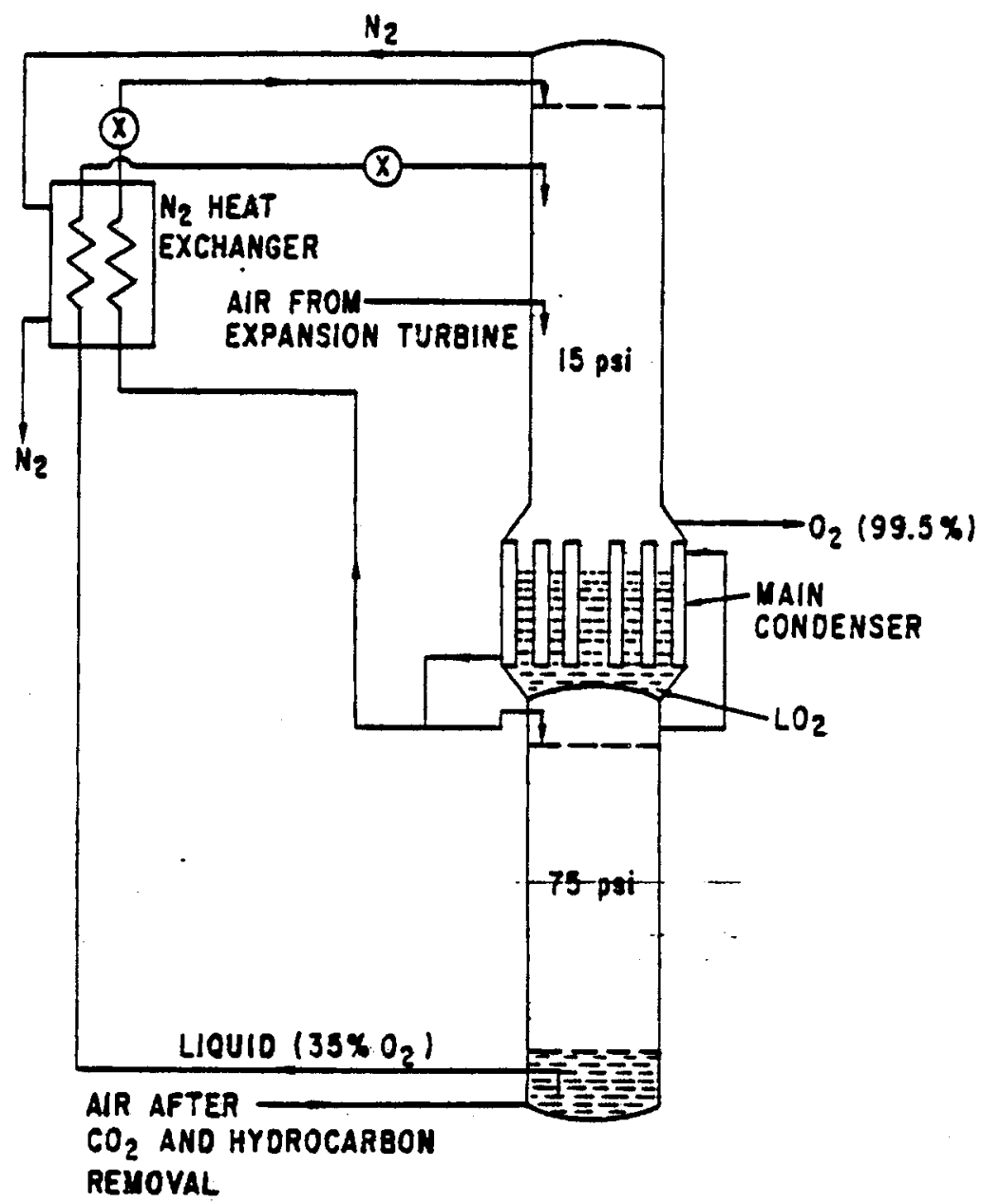

FIGURE A.4 Double-Column Rectifier (Shen 1980)

Heat-Transfer Operations

The major heat-transfer operations in industrial gas production are compressor cooling, distillation (condensing and reboiling), oxygen and nitrogen superheating, and air precooling/product heating. The heating and cooling media for each of these heat-transfer operations are listed in Table A.8. Whenever possible, process streams are used to effect heat exchange to save energy.

Reversing heat exchangers are used for air precooling and product heating. These heat exchangers are a sort of combination recuperator and 
TABLE A.7. Energy Used to Produce Oxygen and Nitrogen (Shen 1980)

Process, Product

\begin{tabular}{l} 
Process, Product \\
\hline $\begin{array}{l}\text { Separation }(a) \\
\text { Oxygen gas }(a) \\
\text { Nitrogen gas }\end{array}$ \\
Liquefaction \\
Liquid oxygen \\
Liquid nitrogen \\
Separation plus liquefaction $(b)$ \\
Liquid oxygen \\
Liquid nitrogen \\
Separation plus pressurization \\
Oxygen gas at 425 psig \\
Nitrogen gas at 200 psig
\end{tabular}

kWhe/ton

278

60

525

582

800

640

395

163

(a) at $70^{\circ} \mathrm{F}$ and 14.7 psia

(b) totals not exact due to rounding

TABLE A.8. Heat-Transfer Media in Air Separation Plants

\begin{tabular}{|c|c|c|}
\hline Operation & Heating Medium & Cooling Medium \\
\hline Compressor Cooling & Waste Heat & Cooling Water \\
\hline $\begin{array}{l}\text { Distillation (Condenser/ } \\
\text { Reboiler) }\end{array}$ & Nitrogen & Oxygen \\
\hline Air Precooling/Product Heating & Air & $\begin{array}{l}\text { Product 0xygen } \\
\text { and Nitrogen }\end{array}$ \\
\hline Nitrogen Superheating & Oxygen & Nitrogen \\
\hline 0xygen Superheating & Air & oxygen \\
\hline
\end{tabular}

regenerator. The air and the product streams flow countercurrent to each other. The product nitrogen and oxygen flow in separate, nonreversing passages, while the waste nitrogen flows through passages that periodically reverse with the air passages. Extended surfaces are typically used to enhance the heat transfer (Shen 1980). 
Heat exchangers are generally compact plate-and-fin exchangers constructed of brazed aluminum. Distillation trays may be either perforated or bubble cap. Construction materials for distillation trays and heat exchangers include aluminum, stainless steel, copper, and copper alloys. Equipment operating below room temperature is assembled in one or more "cold boxes" which are heavily insulated (Shen 1980).

Waste Heat Streams

The only significant source of waste heat from air separation facilities is that rejected by the compressors. It is estimated that about $571,500 \mathrm{Btu} /$ ton of oxygen produced is rejected as hot air at about $250^{\circ} \mathrm{F}$ (Latour 1981 ). If cooling water is used as the sink for the waste heat, it is estimated that about 2200 gallons of cooling water would be discharged per ton of oxygen produced at a temperature about $30^{\circ} \mathrm{F}$ above ambient (Latour, 1981). It is estimated that the compressor cooling water waste stream for a 2000 ton/day oxygen plant is at temperatures between 100 and $120^{\circ} \mathrm{F}$ and a flow rate of about $700,000 \mathrm{lb} / \mathrm{hr}$ (Palmer 1980). Another source estimates that an average of 460 Btu are rejected as waste heat from compressors per pound of oxygen produced (Sittig 1977).

OTHER INDUSTRIAL INORGANICS - SIC 2819

This product category encompasses a large number of inorganic chemicals. One of these, sulfuric acid, is produced in the largest quantity of any chemical in the chemical industry. In this category others that are discussed in this secton are elemental phosphorus and phosphoric acid.

Process Description

The processes grouped in this classification are highly varied; thus, similarities in operation between the production of the various chemicals is uncommon. The manufacturing processes for sulfuric acid, phosphorus and phosphoric acid are described in this section.

Sulfuric Acid

The most common method used to manufacture sulfuric acid is the contact process shown in Figure A.5. In this process, atomized sulfur is fed to the 


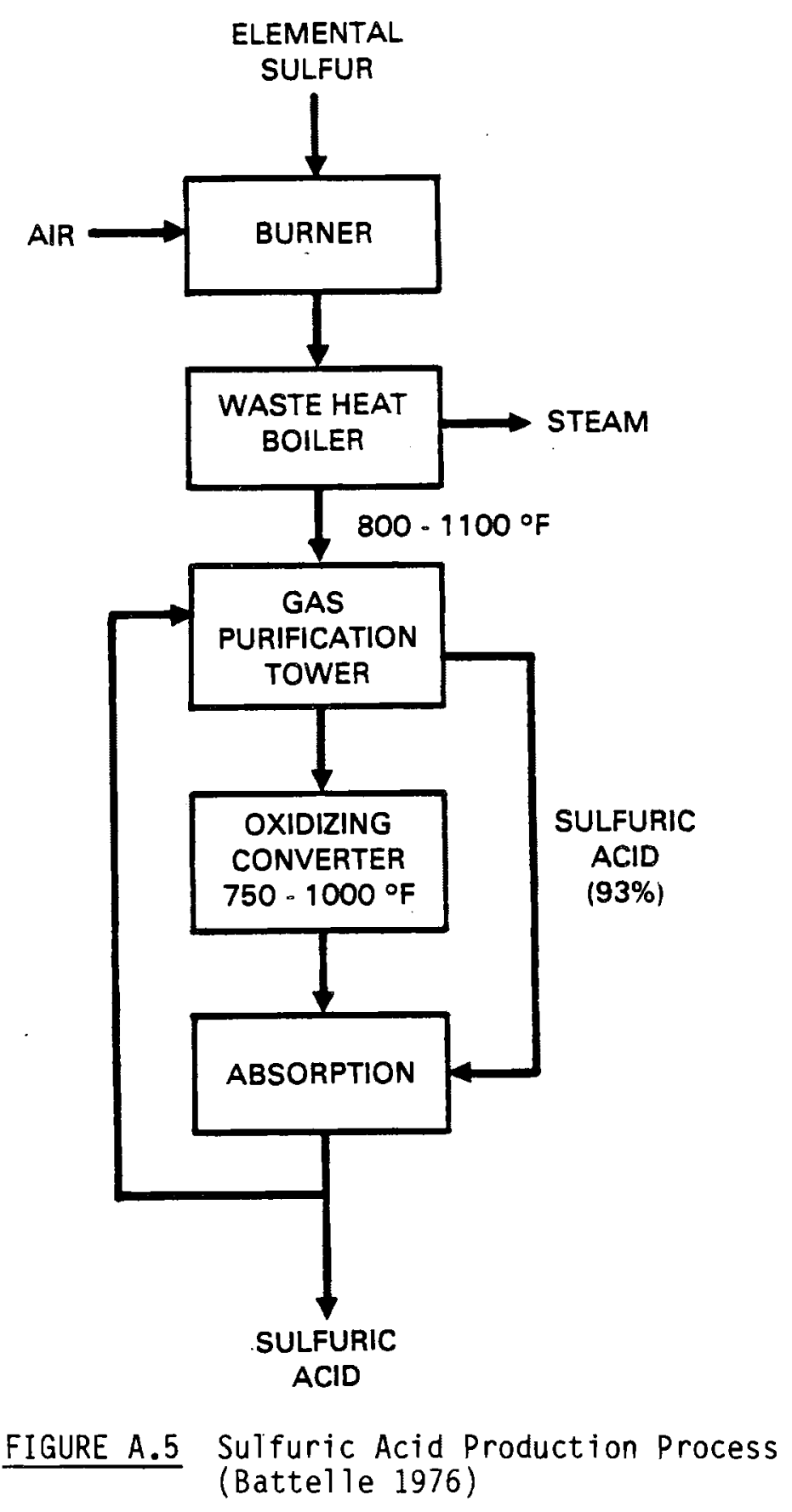

burner along with predried air. In the burner, the sulfur is converted to sulfur dioxide. The exothermic reaction gives off a large quantity of heat. The heat is recovered in waste heat boilers to produce steam for other plant 
operations. The cooled gases from the waste heat boilers flow countercurrent to a stream of concentrated sulfuric acid in a tower for purification and drying. The dried and purified gas stream, which consists of sulfur dioxide, oxygen, and nitrogen, passes through a multi-stage, catalytic converter that oxidizes the sulfur dioxide to sulfur trioxide. The catalytic conversion is also an exothermic reaction. Following conversion, the sulfur trioxide is absorbed in $93 \%$ sulfuric acid recirculating from the gas purification tower to produce concentrated sulfuric acid. Part of the concentrated sulfuric acid product is recycled to the gas purification tower as needed.

\section{Elemental Phosphorus}

Elemental phosphorus is produced exclusively in the U.S. by the electric fusion of phosphate rock, as shown in Figure A.6. Phosphate rock is passed through a rotary kiln or traveling grate at 1400 to $1600^{\circ} \mathrm{F}$ to form spherical nodules that are between one and five centimeters in diameter. Most of the phosphate rock requires little beneficiation because silica, which is the major impurity, is required for efficient furnace operation. Coke and additional silica are dried, screened and fed to the furnace. When the feed reaches its fusion temperature, the calcium in the phosphate rock reacts with the silica, forming a calcium silicate slag. The carbon from the coke acts as a reducing agent to form phosphorus vapor and carbon monoxide from the $\mathrm{P}_{2} \mathrm{O}_{5}$, which is released from the phosphate rock. Iron impurities in the feed react with some of the phosphorus to form molten ferrophosphorus. The calcium silicate slag and the ferrophosphorus are periodically tapped from the furnace.

The gas-phase mixture of phosphorus and carbon monoxide pass through an electrostatic precipitator to remove dust that is carried over from the furnace. The phosphorus is then liquefied in a water-spray tower and the carbon monoxide is drawn off for use as a fuel in the nodulizing process.

\section{Phosphoric Acid}

Figure A.7 shows the manufacturing steps in converting phosphate rock to phosphoric acid by digestion with sulfuric acid. This is commonly referred to as the wet-acid process. Following washing and floatation to concentrate the 


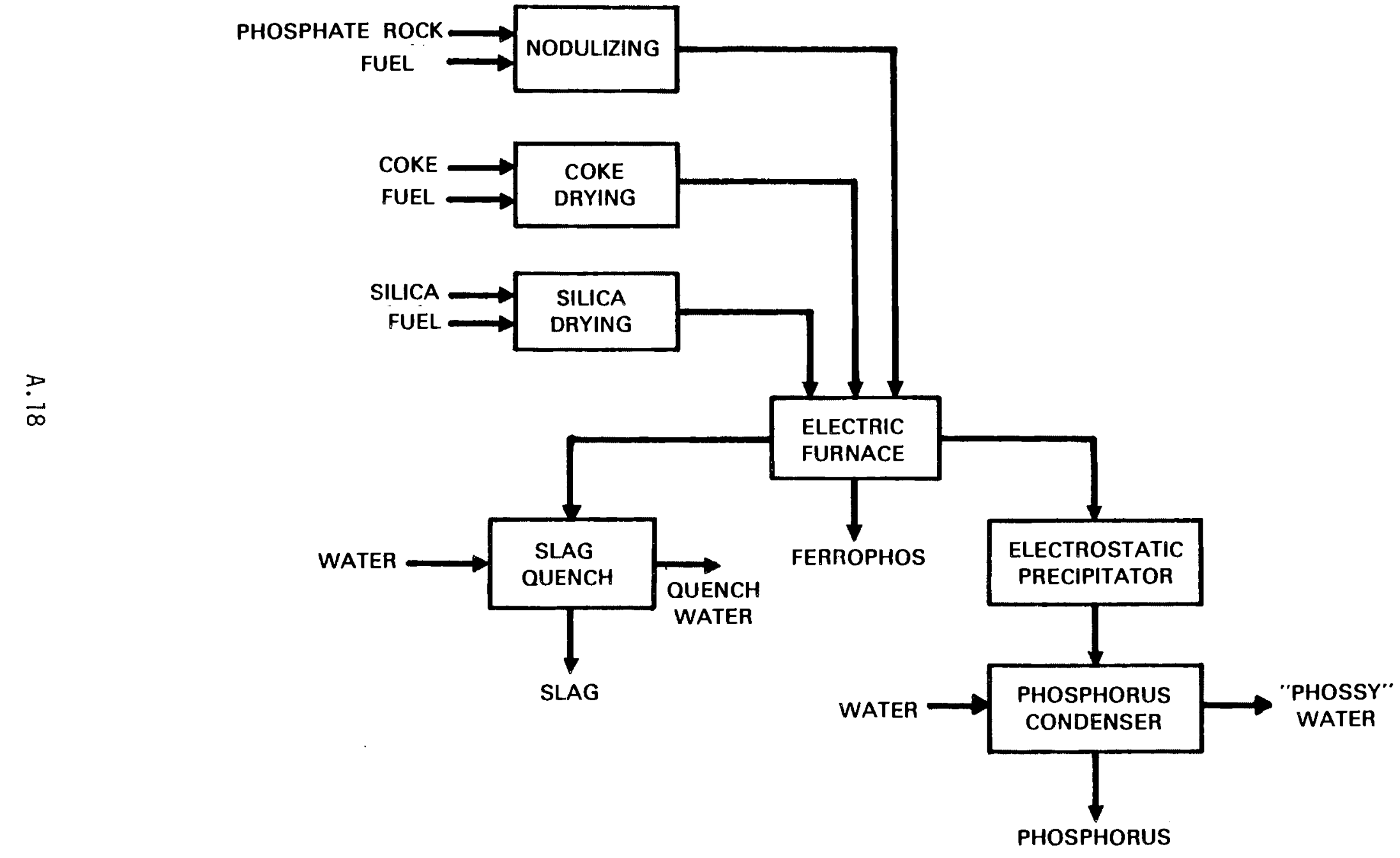

FIGURE A.6. Phosphorus Flow Diagram (A.D. Little 1976) 


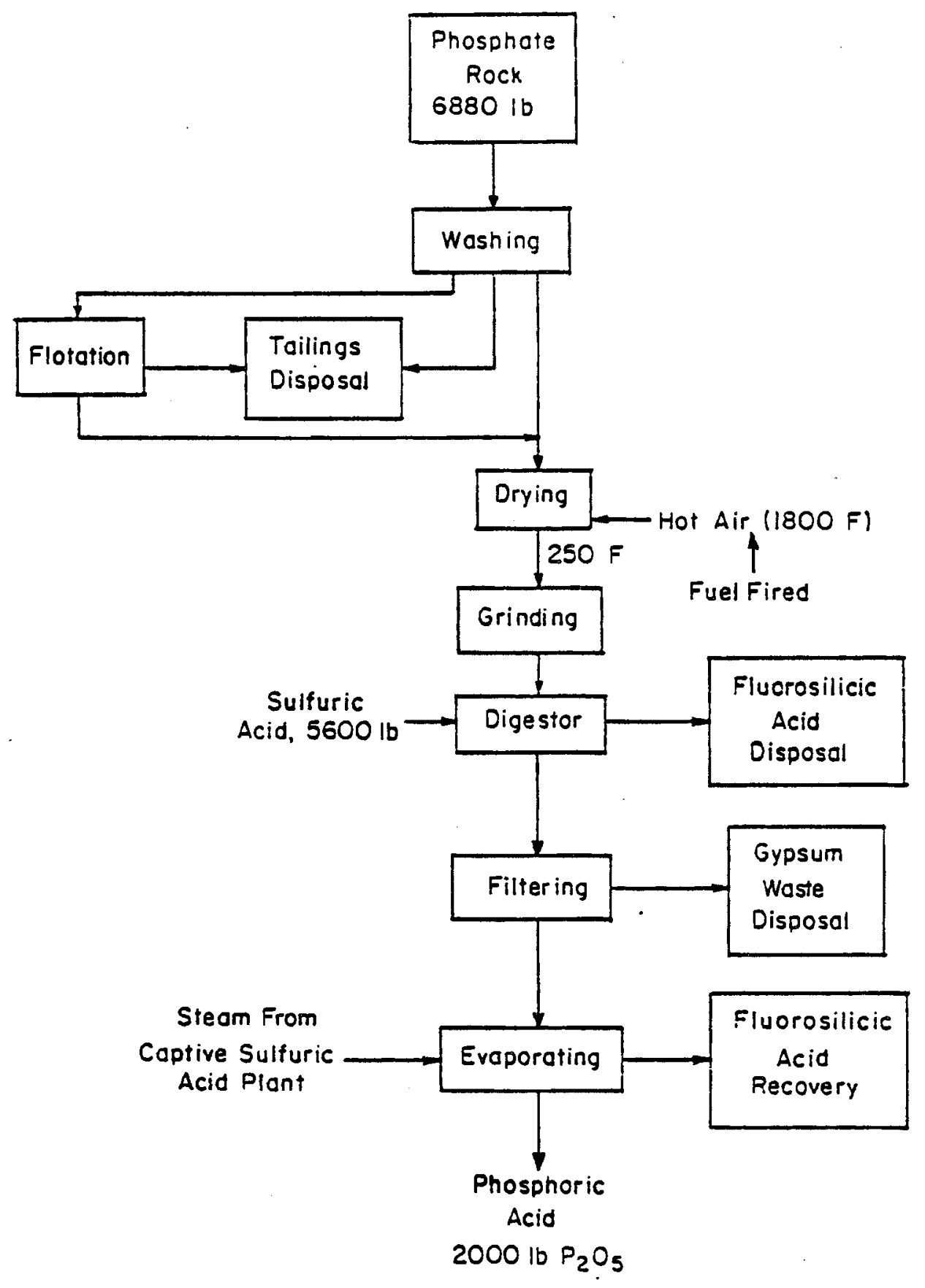

FIGURE A.7. Phosphoric Acid Production (Batte1le 1976) 
phosphorus, the ore is dried in rotary dryers. The ore is then ground and fed to the digestors where it is first treated with dilute phosphoric acid. The phosphate rock and the dilute acid react as follows:

$$
\mathrm{Ca}_{3}\left(\mathrm{PO}_{4}\right)_{2}+4 \mathrm{H}_{3} \mathrm{PO}_{4} \longrightarrow 3 \mathrm{Ca}\left(\mathrm{H}_{2} \mathrm{PO}_{4}\right)_{2}
$$

This solution is then acidified with strong sulfuric acid, which reacts with the monocalcium phosphate to precipitate gypsum and to form phosphoric acid as follows:

$$
3 \mathrm{Ca}\left(\mathrm{H}_{2} \mathrm{PO}_{4}\right)_{2}+3 \mathrm{H}_{2} \mathrm{SO}_{4} \longrightarrow 3 \mathrm{CaSO}_{4}+6 \mathrm{H}_{3} \mathrm{PO}_{4}
$$

Total residence time in the digestor is about six hours.

The slurry from the digestor is processed through a rotary vacuum filter to separate the gypsum for subsequent disposal. The filtrate is phosphoric acid with a concentration of about 30 to $32 \%$ (A. D. Little 1976). The weak acid is concentrated to the desired strength by evaporating the water in multieffect evaporators.

Plants range in capacity from about 50 tons/day to 2000 tons/aay with the typical plant producing about 780 tons/day (Palmer 1980).

Heat-Transfer Operations

The major heat-transfer operations are discussed below for each of the processes described earlier.

\section{Sulfuric Acid}

Sulfuric acid production requires only a modest amount of electricity for moving gaseous and liquid streams. Both the sulfur burning process and the catalytic conversion process are exothermic, making the overall process a net generator of heat. The majority of the heat-transfer operations recover heat from the product following these exothermic reactions. It is estimated that potentially $3.4 \times 10^{6}$ Btu could be recovered per ton of sulfuric acid produced (Bodine 1980). Heat can be recovered in four places:

1. after the sulfur burner

2. after the first converter pass

3. following catalytic conversion

4. from the hot acid prior to storage. 
The hot gases from the sulfur burner contain about $10 \%$ by volume sulfur dioxide and are at temperatures of 1740 to $1830^{\circ} \mathrm{F}$ (Bodine 1980). The hot gases are used to produce steam in a fire-tube waste-heat boiler. The steam generated is of sufficient quality to produce power. In more modern plants, most of the steam is used to power pumps and blowers, replacing the need for electricity. After providing for fluid transport, steam is still available for export from the plant.

Heat may be recovered from the gas stream following the first pass through the catalytic converter either by a fire-tube waste-heat boiler or an extended surface superheater. Following the converter, the process gases are further cooled in extended surface economizers. The hot acid product is cooled prior to storage in water-cooled heat exchangers.

\section{Elemental Phosphorus}

The major heat-transfer operations in phosphorus production include phosphate ore nodulizing, feedstock drying, phosphorus reduction, slag quenching, and phosphorus condensing. The most energy-intensive process is phosphorus reduction in the electric furnace. About $12,500 \mathrm{kWh} / \mathrm{ton}$ of phosphorus are required for furnace operation (Battelle 1976). In addition, the reduction of $\mathrm{P}_{2} \mathrm{O}_{5}$ is exothermic, adding more heat to the furnace.

In ore nodulizing and feedstock drying the heat-transfer is by direct contact between the hot combustion gases and the ore or feedstock. The carbon monoxide generated in the furnace is burned to provide the heat for nodulizing. Any fuel can be burned to dry the coke and the silica.

Slag quenching and phosphate cooling involve direct contact with cooling water. Lastly, cooling water is used to cool electrode clamps in the furnace and to prevent corrosion of the slag taphole. A water spray cools the outer shell of the furnace.

Phosphoric Acid

Two steps in the producton of phosphoric acid require the addition of process heat. These are the drying of $\mathrm{P}_{2} \mathrm{O}_{5}$ concentrate prior to grinding, and the evaporation of the water to produce concentrated phosphoric acid. The 
digestion reaction is strongly exothermic, requiring cooling to maintain the required process temperature.

Any fuel can be used in the concentrate drying process. About $1.21 \mathrm{x}$ $10^{6}$ Btu are required per ton of $\mathrm{P}_{2} \mathrm{O}_{5}$ (Battelle 1976). Steam is used for heating and evacuating the multiple-effect evaporators used to concentrate the acid. This step requires about 2100 pounds of steam at 90 psi and $320^{\circ} \mathrm{F}$ per ton of $\mathrm{P}_{2} \mathrm{O}_{5}$ (Battelle 1976). The steam used in the concentration step is the excess steam produced by the captive sulfuric acid plant that provides the acid used in the digestor.

Waste Heat Streams

The majority of the waste heat in the manufacture of the inorganic chemicals described in the preceding sections is in the form of waste water. Heat is typically recovered from high-temperature product gases for steam generation or for use as process heat. The waste heat streams for each of the processes are discussed below.

\section{Sulfuric Acid}

No significant waste heat streams have been identified in the sulfuric acid production process, despite the fact that it is a net generator, rather than user, of heat. In this process, all of the streams containing waste heat are heat exchanged to successively heat water and then to produce steam. To summarize, the hot acid product is cooled in water-cooled heat exchangers. The water is then routed to the economizers that cool the product gases prior to the absorption process. From there, the steam or water is routed to either a waste-heat boiler or a superheater which recovers additional heat after the first pass through the converter. In addition, the heat in the product stream exiting the sulfur burner is recovered in a waste-heat boiler.

\section{Elemental Phosphorus}

The significant sources of waste heat in elemental phosphorus production are the exit gases from the nodulizing $k i l n$ and the feedstock dryers, and the waste waters from nodulizer off-gas scrubbing, slag quenching, and phosphorus condensing. Table A.9 gives the nodulizer exit gas parameters for a sample 
TABLE A.9. Nodulizer Exit Gas Parameters for Sample Plant (Cox 1979)

$\begin{array}{ll}\text { Temperature } & 1022^{\circ} \mathrm{F} \\ \text { Flow } & 140,000 \text { ACFM } \\ \text { Dust Load } & 450 \mathrm{lbs} / \mathrm{hr} \\ \text { Corrosive Components } & \mathrm{HF}, \mathrm{SO}\end{array}$

plant. No information was available for the exhausts from the feedstock dryers.

The exit gases from the nodulizing process are at a temperature of about $1022^{\circ} \mathrm{F}$ prior to the scrubbers (Cox 1979). The gases are laden with dust and may contain corrosive components such as hydrogen fluoride and sulfur trioxide. The information in Table A.9 was provided for one plant with a traveling grate nodulizer.

Since the nodulizer exhaust gas is dusty and contains fluorides, pollution control equipment must be used prior to venting to the atmosphere. Typically, scrubbers are used and the water is recycled. The purge stream from the recycle is contaminated with phosphates, sulfates, fluorides, and suspended solids and is acidic (A. D. Little 1976).

The slag quench water is slightly alkaline and contains phosphates, sulfates, fluorides and suspended solids. The "phossy" water from the phosphorus condenser contains a small amount of phosphorus in suspension as a finely divided solid.

The volumes and content of the three waste cooling water streams vary from plant to plant. Table A.10 provides an estimate of the characteristics of the waste water for a 100,000 ton/year phosphorus plant.

Phosphoric Acid

Sources of waste heat from phosphoric acid production include dryer exhausts, digestor vent gas, and evaporation system vent gas. The exhaust gases are scrubbed with a water spray to remove any gaseous fluorine. No information on the temperatures, volumes, or heat contents of these waste streams was found. 
TABLE A.10 Phosphorus Wastewater Characteristics (A. D. Little 1976)

Basis: 100,000 tpy production (as $\mathrm{P}_{4}$ )

330 operating days/year

\begin{tabular}{|c|c|c|c|c|c|c|c|c|}
\hline Wastewater Characteristics & $\begin{array}{l}\text { Nodul } \\
\text { Scrubber } \\
\text { (mg/1) }\end{array}$ & $\begin{array}{l}\text { izer } \\
\text { Liquor* } \\
\text { (1b/day) }\end{array}$ & $\begin{array}{r}\text { Pho } \\
\text { Conde } \\
\text { Other } \\
\text { (mg/1) }\end{array}$ & $\begin{array}{l}\text { horus } \\
\text { er Plus } \\
\text { ssy Water } \\
\text { (1b/day) }\end{array}$ & $\begin{array}{r}\text { S } \\
\text { Quench } \\
\text { (mg/1) }\end{array}$ & $\begin{array}{l}9 \\
\text { lb/day) }\end{array}$ & $\begin{array}{l}\text { Combined } \\
(\mathrm{mg} / \mathrm{l})\end{array}$ & $\begin{array}{c}\text { Wastewater } \\
\text { (1b/day) }\end{array}$ \\
\hline Total suspended solids & 280 & 5,100 & 135 & 8,190 & 820 & 12,450 & 273 & 25,740 \\
\hline Phosphorus $\left(\mathrm{P}_{4}\right)$ & - & - & 90 & 5,460 & - & - & 58 & - \\
\hline Phosphate $\left(\mathrm{PO}_{4}\right)$ & 70 & 1,270 & 220 & 13,350 & 40 & 610 & 162 & 15,230 \\
\hline Sulfate $\left(\mathrm{SO}_{4}\right)$ & 1,200 & 21,830 & - & - & 3,000 & 45,560 & 717 & 67,390 \\
\hline Fluoride (F) & 730 & 13,280 & 270 & 16,380 & 180 & 2,730 & 344 & 32,390 \\
\hline Total Acidity (as $\mathrm{CaCO}_{3}$ ) & 2,000 & 36,380 & - & - & - & - & 351 & 33,040 \\
\hline Total Alkalinity (as $\mathrm{CaCO}_{3}$ ) & - & - & - & - & 220 & 3,340 & - & - \\
\hline Wastewater Flow Rate & \multicolumn{2}{|c|}{$2.18 \mathrm{mgd}$} & \multicolumn{2}{|c|}{$7.27 \mathrm{mgd}$} & \multicolumn{2}{|c|}{$1.82 \mathrm{mgd}$} & \multicolumn{2}{|c|}{$11.27 \mathrm{mgd}$} \\
\hline
\end{tabular}

*Assumes scrubber liquor is recirculated with a $10 \%$ blowdown. 
PLASTICS MATERIALS AND RESINS - SIC 2821

The manufacture of plastics and resin materials in the United States involves the production of thermoplastic and thermosetting polymers and thermoplastic elastomers. Four of these account for $60 \%$ of the total production of plastics and resins (Battelle 1976):

- low-density polyethylene (LDPE)

- high-density polyethylene (HDPE)

- polyvinyl chloride (PVC)

- polystyrene.

The manufacturing processes, heat-transfer operations and waste heat streams are analyzed below for these four representatives of the plastics industry. Process Description

Low-Density Polyethylene

LDPE is the largest-volume plastic material manufactured and is the largest single product produced from ethylene. There are two basic processes used to manufacture LDPE: the autoclave reactor process and the tubular reactor process. With the exception of the type of reactor, these processes are similar. Both are high-pressure processes, with pressures ranging from 15,000 to 50,000 psi (Battelle 1976). The energy consumption of each process is essentially the same.

Tubular reactors have tubes with an inner diameter of about 1 inch and may be up to 2000 feet long. The material residence time in the reactor may be up to 45 seconds at a production rate of 20 to $40 \mathrm{million} 1 \mathrm{~b} / \mathrm{yr}$ (Gaines 1980). Large autoclave reactors may have internal diameters up to 2 feet with an outer diameter three or four times as large. The length-to-diameter ratio may vary from 2:1 to $20: 1$. Reactors can produce from 5 to $15 \mathrm{million} 1 \mathrm{bs} / \mathrm{yr}$ (Gaines 1980). Plants range in size from 100 tons/day to 1000 tons/day with the average plant at about 500 tons/day (Palmer 1980). Figure A.8 shows the basic steps in LDPE production.

Feed materials (ethylene, an initiating agent and a chain transfer agent) are compressed in stages and mixed with recycled ethylene. The compressed feed 


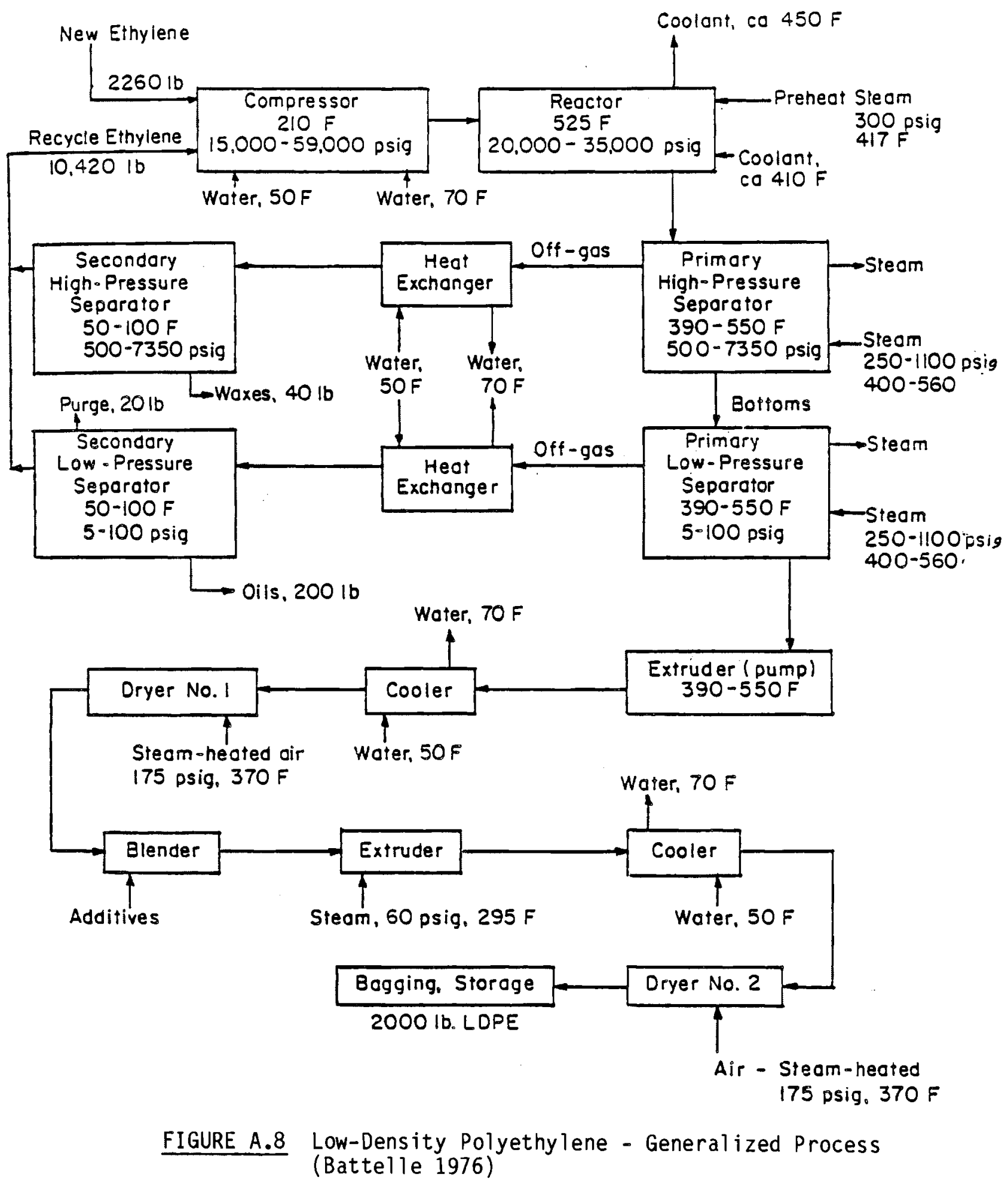

enters the reactor at pressures of from 15,000 to 50,000 psi. In the reactor, the materials are heated to 230 to $340^{\circ} \mathrm{F}$ to initiate polymerization. The normal conversion of ethylene to LDPE is about 22-30\% per pass for tubular 
reactors and 10 to $20 \%$ per pass for autoclave reactors (UTC 1980). The polyethylene is then separated from the unpolymerized ethylene in a series of heated separators. About 95\% of the unreacted ethylene is removed in the highpressure separator and the remainder is removed in the low-pressure separator (Battelle 1976). The ethylene from the separators is cooled, dewaxed and recycled. The molten polymer from the low-pressure separator is extruded and pelletized, then cooled, dried and stored.

High-Density Polyethylene

High-density polyethylene is manufactured by one of several low-pressure processes that are completely different than the high-pressure processes discussed earlier. Pressures in these processes range from about 15 to 3000 psi (UTC 1980). Nearly complete polymerization occurs in a single pass in these processes. Three commercial processes are employed: (1) the slurry process, (2) the solution process, and (3) the gas-phase process. In the solution process both the quantity of the hydrocarbon solvent and the process temperature are maintained at a sufficiently high level to keep the polymer in solution. The slurry processes use a smaller amount of solvent and lower temperatures, and the polymer is formed as a dispersed solid in a slurry. Gas-phase processes have been developed in which no solvent is required; however, little HDPE is produced by the gas-phase methods.

Figure A.9 outlines the basic steps in the solution process. In the solution process, the solid catalyst in a hydrocarbon diluent is introduced into an autoclave reactor containing ethylene and the diluent. Reactor conditions range from 250 to $350^{\circ} \mathrm{F}$ at 300 to 500 psia (UTC 1980). The polymer and diluent are separated by steam stripping the diluent, leaving a solid polyethylene and water mixture. The diluent and the unpolymerized ethylene are recycled to the reactor. The solid polyethylene is separated from the water and then dried and extruded to form pellets.

In the slurry process (shown in Figure A.10), high-purity ethylene, a highly active catalyst, and a diluent are fed to the reactor. Reactor conditions range from 68 to $212^{\circ} \mathrm{F}$ at 100 to 500 psia. Flow velocities vary 


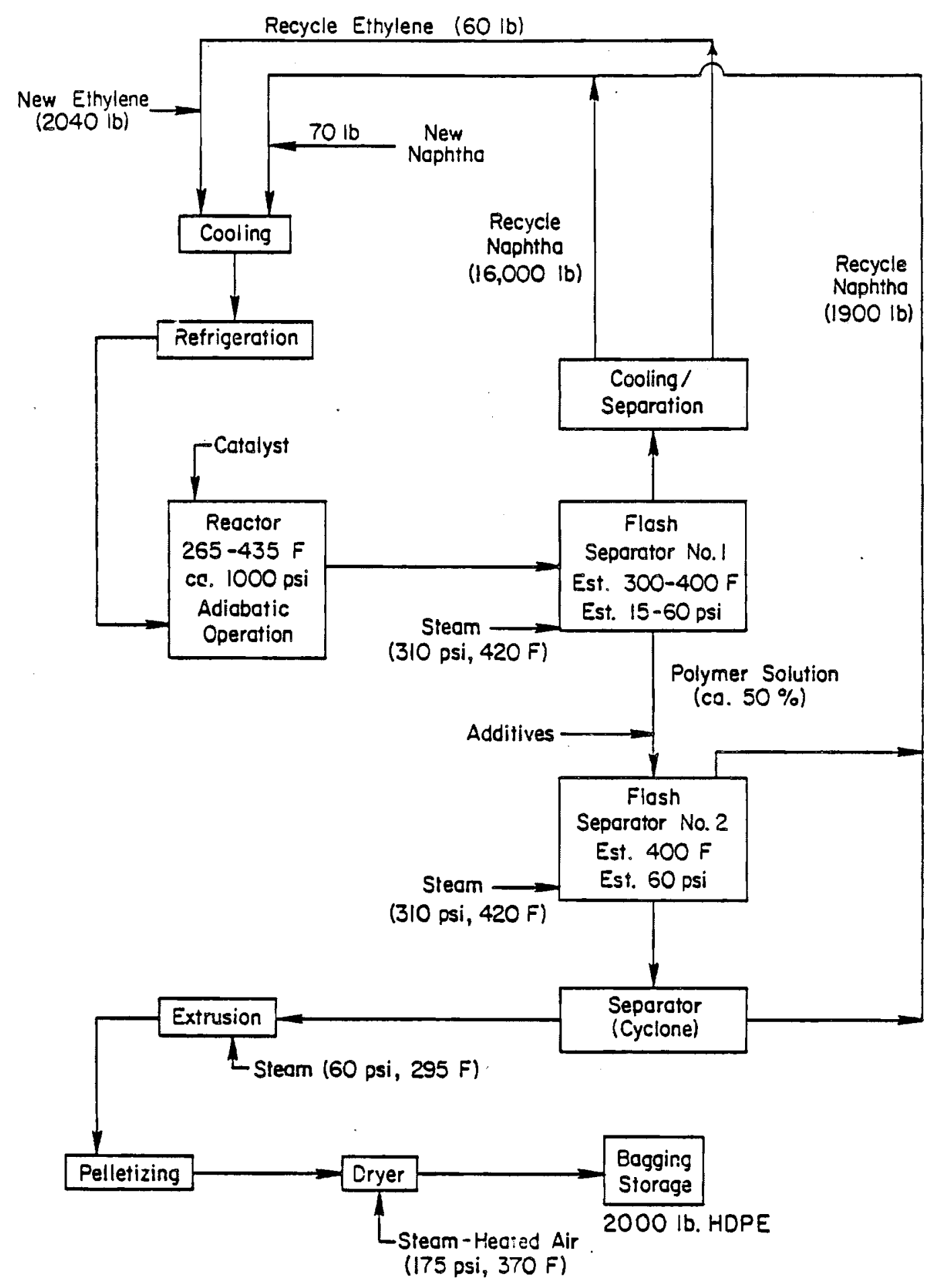

FIGURE A.9. High-Density Polyethylene - Stamicarb Solution Process 


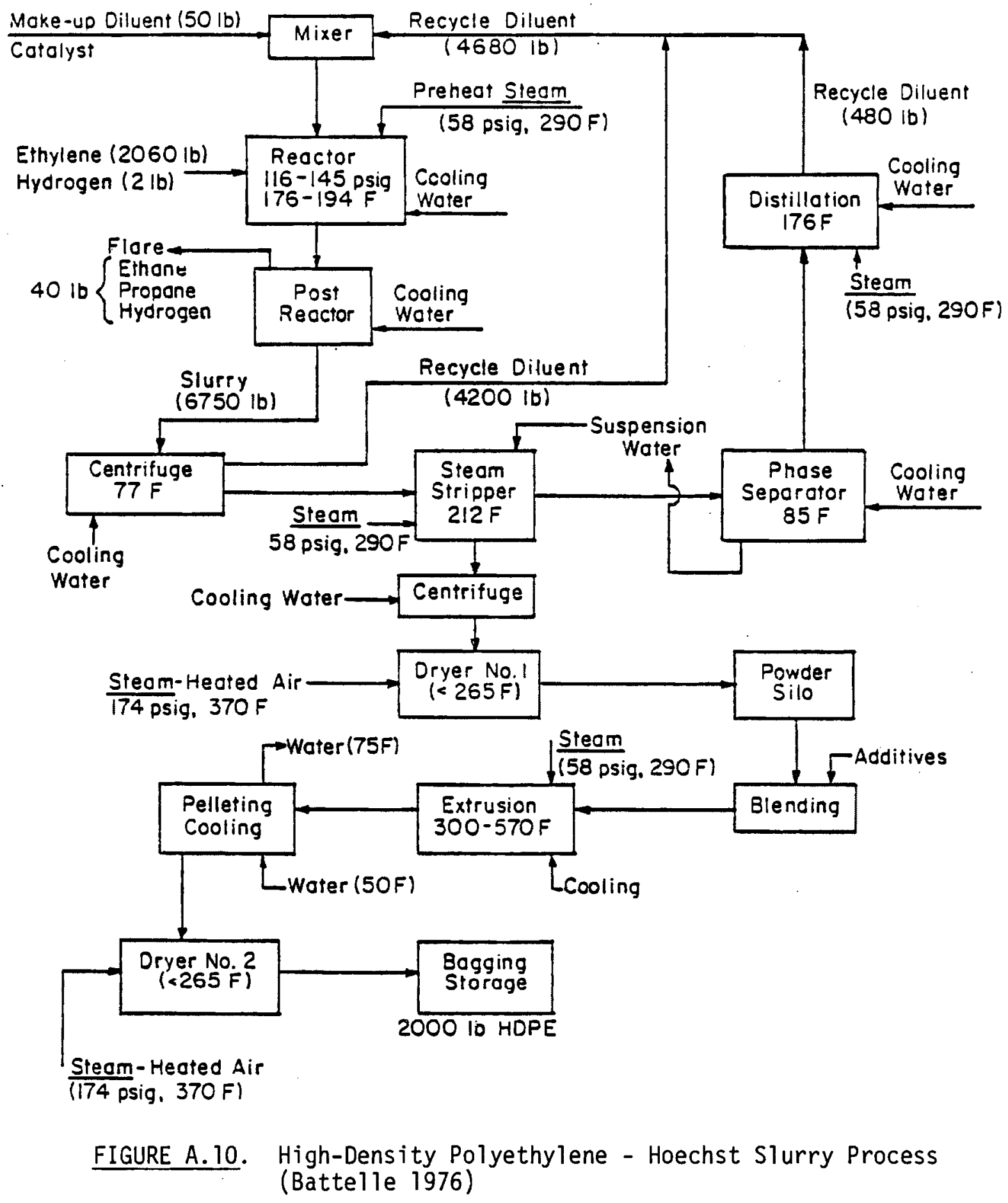

from 10 to $30 \mathrm{ft} / \mathrm{sec}$ (UTC 1980). The product and diluent stream withdrawn from the reactor are separated by flashing off the solvent at high temperatures and low pressures. The solvent is recycled back to the reactor. The product is 
sent to auger dryers to remove remaining solvent and the product is sold in bulk, granular or pelletized form.

\section{Polyvinyl Chloride}

PVC is manufactured by one of four processes: (1) suspension, (2) bulk, (3) solution, and (4) emulsion. Suspension polymerization is used to produce an estimated 85 to $86 \%$ of the total PVC (Battelle 1976, UTC 1980). This process is shown schematically in Figure A.11.

The vinyl chloride monomer, finely dispersed in deionized water, is charged to the reactor which is agitated and heated to between 113 and $131^{\circ} \mathrm{F}$ (UTC 1980). When 90\% polymerization is achieved, the mixture is transferred to a dump tank and the unreacted monomer is stripped and recovered for recycle. Most of the water is removed by centrifugation and the resin is dried in a rotary dryer with hot air. A cyclone separator is used to remove the product from the hot air stream exiting the dryer. Stabilizers and plasticizers are added in the blender by tumbling. Some of the PVC is further processed by melting, pelletizing and drying.

\section{Polystyrene}

Continuous bulk polymerization is the most common process for producing polystyrene. Figure A.12 shows the major steps in the bulk polymerization process. Styrene monomer is sent to a prepolymerizer where it is heated, forming a viscous solution of polymer in styrene. Prepolymerization, which may take place in a stirred reactor, tower or autoclave reactor, may achieve as little as $11 \%$ conversion or as much as $85 \%$ depending on the process. The viscous solution is pumped to the top of a tower and passes down through increasing temperature zones. The reaction is completed at the bottom of the tower at a temperature of about $390^{\circ} \mathrm{F}$ (Gaines 1980).

The molten polystyrene is pumped from the bottom of the tower through dies to form strands. Unreacted styrene is vacuum-flashed and recovered for recycle. The strands are cooled and chopped. 


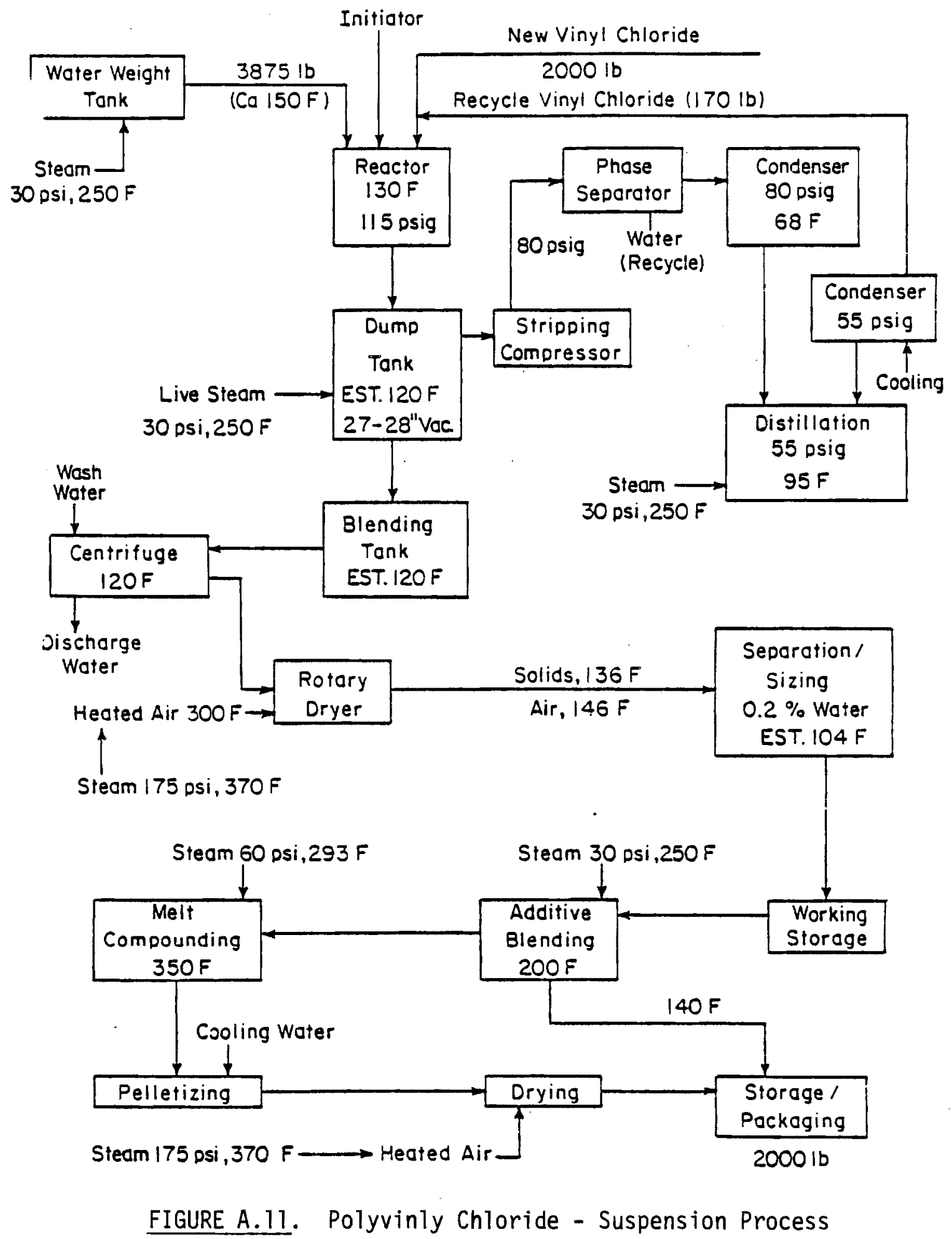




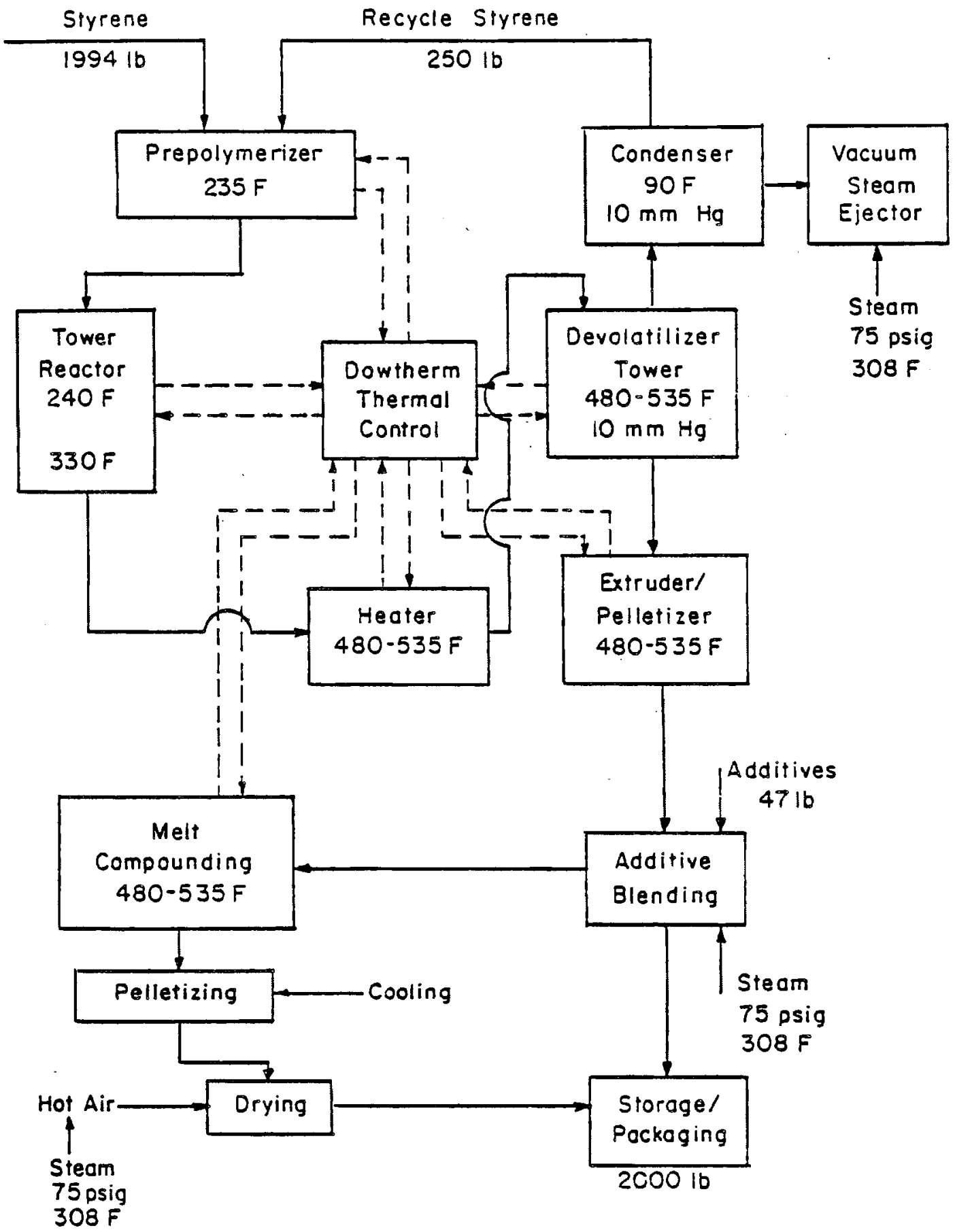

FIGURE A.12. Polystyrene - Bulk Polymerization Process (Battelle 1976)

\section{Heat-Transfer Operations}

The major heat-transfer operations for each of the processes described above are discussed below. 


\section{Low-Density Polyethylene}

The major heat-transfer operations in LDPE production are listed in Table A.10 along with the medium used for heating or cooling. Ethylene compressors are water cooled to prevent premature polymerization. Ethylene is initially preheated to a temperature of 230 to $340^{\circ} \mathrm{F}$ via a heat transfer fluid such as Dowtherm in the reactor jacket. As the reaction proceeds, cooling must be provided to the jacket to prevent explosive decomposition. As an alternative to jacketed cooling, multiple feed points may be provided in a tubular reactor and the new feed is heated by the excess heat of polymerization.

Heat must be added to the separators to maintain polyethylene in its molten state and to decrease the solubility of the gaseous ethylene in the product. Steam-jacketed separators are typically used to avoid excessive product heat loss. The off-gas from the primary separators are cooled to between 50 and $100^{\circ} \mathrm{F}$ and are passed through the secondary separators. The ethylene removed from the separators is air or water cooled.

Initially heat is added to the extruder to maintain polymer fluidity; however, heat generated by the extrusion process itself must subsequently be removed by direct-contact water cooling. Following cooling, the wet pellets are dried in a hot air dryer. The hot air is steam heated.

TABLE A.10. Heat-Transfer Operations in LDPE Production

\begin{tabular}{ll}
\multicolumn{1}{c}{ Operation } & Heating/Cooling Medium \\
\cline { 1 - 1 } Compressor Cooling & water \\
Ethylene Preheating & steam or Dowtherm ${ }^{(0)}$ \\
Reactor Cooling & coolant \\
Separator Heating & steam \\
Separator Off-Gas Cooling & water or air \\
Extrusion & steam \\
Extruded LDPE Cooling & water \\
Extruded LDPE Drying & steam
\end{tabular}

Trademark of the Dow Chemical Company, Midland, Michigan. 


\section{High-Density Polyethylene}

The major heat-transfer operations in HDPE production by the slurry process are listed in Table A.11 and those in the solution process are shown in Table A.12.

A11 the process heat added in the slurry process is in the form of steam. It is estimated that about 1650 pounds of low-pressure steam and 600 pounds of medium-pressure steam are required to produce one short ton of HDPE (Battelle 1976). In the reactor, steam is initially used to promote polymerization. Then, cooling water must be used to maintain the reactor conditions at about $185^{\circ} \mathrm{F}$ and $130 \mathrm{psi}$ (Battelle 1976). The remaining process equipment is maintained at the temperature levels listed in Table A.13.

TABLE A.11. Major Heat-Transfer Operations in HDPE Production by the Slurry Process (Battelle 1976)

\section{Operation}

Ethylene Preheating

Reactor Cooling

Centrifuge Cooling

Steam Stripping

Diluent Distillation

- Heating

- Cooling

Phase Separation

Drying

Extrusion

- Heating

- Cooling

Drying
Heat ing/Cooling Medium

Steam

Water

Water

Steam

Steam

Water

Water

Air (Steam

Heated)

Steam

Water

Air (Steam Heated)
Quantity

$550 \mathrm{1b} /$ ton HDPE

(60 psi, $295^{\circ} \mathrm{F}$ )

$700 \mathrm{1b} /$ ton HDPE

$\left(60 \mathrm{psi}, 295^{\circ} \mathrm{F}\right)$

$100 \mathrm{1b} /$ ton HDPE

$\left(60 \mathrm{psi}, 295^{\circ} \mathrm{F}\right)$

$5001 \mathrm{~b} /$ ton HDPE

(175 psi, $370^{\circ} \mathrm{F}$ )

$3001 \mathrm{~b} /$ ton HDPE

(60 psi, 295 F)

$100 \mathrm{lb} /$ ton HDPE

(175 psi, $370^{\circ} \mathrm{F}$ ) 
TABLE A.12. Heat-Transfer Operations in HDPE Production by the Solution Process (Battelle 1976)

$\frac{\text { Operation }}{\text { Reactor Cooling }}$
Flash Separator
Extruder
- Heating
- Cooling
Dryer

\begin{tabular}{|c|c|}
\hline Heating/Cooling Medium & Quantity \\
\hline Water & -- \\
\hline Steam & $\begin{array}{l}1900 \mathrm{lb} / \text { ton HDPE } \\
\left(310 \mathrm{psi}, 420^{\circ} \mathrm{F}\right)\end{array}$ \\
\hline Steam & $\begin{array}{l}300 \mathrm{lb} / \text { ton } \mathrm{HDPE} \\
\left(60 \mathrm{ps}, 295^{\circ} \mathrm{F}\right)\end{array}$ \\
\hline Water & -- \\
\hline Steam & $\begin{array}{l}100 \mathrm{lb} / \text { ton } \mathrm{HDPE} \\
\left(175 \mathrm{psi}, 370^{\circ} \mathrm{F}\right)\end{array}$ \\
\hline
\end{tabular}

TABLE A.13. Unit Operation Temperature Levels (Battelle 1976)

\begin{tabular}{l}
\multicolumn{1}{c}{ Equipment } \\
\hline Centrifuge \\
Steam Stripper \\
Distillation \\
Dryers \\
Extrusion
\end{tabular}

\begin{tabular}{c}
$\begin{array}{c}\text { Temperature } \\
\left({ }^{\circ} \mathrm{F}\right)\end{array}$ \\
\hline 77 \\
212 \\
176 \\
265 \\
$300-570$
\end{tabular}

Polyvinyl Chloride

All process heat in the suspension process is added in the form of low- or medium-pressure steam. Since the polymerization reaction is exothermic, heat must be removed from the reactor by cooling water. The heat-transfer operations, heat-transfer media and quantities of steam used per ton of product produced are listed in Table A.14:

\section{Polystyrene}

Although there are several places in the production process where process heat is required, much of the heat is provided by the exothermic polymerization process. This heat is transferred to the operations which use heat through a 
TABLE A.14. Heat-Transfer Operations in the Production of Polyvinyl Chloride by the Suspension Process (Battelle 1976)

\begin{tabular}{|c|c|c|}
\hline Operation & Heating/Cooling & Quantity \\
\hline \multicolumn{3}{|l|}{ Reactor } \\
\hline - Initiation & Steam & $\begin{array}{l}450 \mathrm{lb} / \text { ton } \mathrm{PVC} \\
\left(30 \mathrm{psi}, 250^{\circ} \mathrm{F}\right)\end{array}$ \\
\hline - Cooling & Water & \\
\hline $\begin{array}{l}\text { Vinyl Chloride Stripping/ } \\
\text { Distillation }\end{array}$ & Steam & $\begin{array}{l}71 \mathrm{~b} / \text { ton } \mathrm{PVC} \\
\left(30 \mathrm{psi}, 250^{\circ} \mathrm{F}\right)\end{array}$ \\
\hline Rotary Drying & $\begin{array}{l}\text { Air (Steam } \\
\text { Heated) }\end{array}$ & $\begin{array}{l}5001 \mathrm{~b} / \text { ton } \mathrm{PVC} \\
\left(175 \mathrm{psi}, 370^{\mathrm{F}}\right)\end{array}$ \\
\hline
\end{tabular}

Dowtherm ${ }^{\circledR}$ distribution system. Some additional heat in the form of steam is required beyond that provided by the heat of the polymerization reaction. The major heat-transfer operations in the production of polystyrene by the bulk process are listed in Table A.15.

Waste Heat Streams

Most of the waste heat in the production of plastics is in the form of cooling water and process water since the polymerization reactions are

TABLE A.15. Heat-Transfer Operations in the Production of Polystyrene by the Bulk Process

(Battelle 1976)

\begin{tabular}{|c|c|c|}
\hline Operation & Heating/Cooling & Quantity \\
\hline Reactor Cooling & Water & - \\
\hline Heating Dowtherm & Steam & $\begin{array}{l}560 \mathrm{lb} / \text { ton } \\
\left(482 \mathrm{psi}, 575^{\circ} \mathrm{F}\right)\end{array}$ \\
\hline Blending & Steam & $\begin{array}{l}100 \mathrm{lb} / \text { ton } \\
\left(30 \mathrm{psi}, 250^{\circ} \mathrm{F}\right)\end{array}$ \\
\hline Extruding/Pelletizing & Water & \\
\hline Drying & Steam & $\begin{array}{l}100 \mathrm{lb} / \text { ton } \\
\left(175 \mathrm{psi}, 370^{\circ} \mathrm{F}\right)\end{array}$ \\
\hline
\end{tabular}


exothermic and require little heat input. The LDPE, HDPE and PVC processes do not appear to recover the heat of reaction for use in other parts of the plant. The production of polystyrene makes extensive use of the heat of polymerization to provide heat elsewhere in the process.

\section{Low-Density Polyethylene}

One source estimates that about $6 \times 10^{6} \mathrm{lb} / \mathrm{hr}$ of cooling water is rejected at temperatures between 100 and $120^{\circ} \mathrm{F}$ for a 1000 ton/day LDPE plant (Palmer 1980). The sources of this cooling water are the reactors and the compressors. It is estimated that between 35,000 and 44,000 gallons of cooling water are required per ton of LDPE produced (UTC 1980). Another source states that the reactor coolant is at a temperature of $380^{\circ} \mathrm{F}$ and that the heat of reaction is $3.3 \times 10^{6}$ Btu per ton of LDPE (Sittig 1977).

\section{High-Density Polyethylene}

In the production of HDPE, process temperatures are much lower than those in LDPE production, thus the cooling water from this process has little availability and waste heat recovery would not be feasible (Sittig 1977). It is estimated that between 50,000 and 59,000 gallons of c00ling water are required per ton of HDPE produced (UTC 1980).

\section{Polyvinyl Chloride}

As with HDPE production, process temperatures are typically low in the manufacture of PVC. It is estimated that about 17,000 gallons of cooling water are required per ton of PVC produced (UTC 1980).

\section{Polystyrene}

Much of the heat generated in the reactors is recovered and reused in other process operations. No estimates on the volume or the temperature of the cooling water were available. 
ORGANIC FIBERS - SIC 2824

This SIC code contains non-cellulosic synthetic organic fibers, including polyester, nylon, and acrylic fibers. Table A.16 shows the 1972 production and energy consumption for selected SIC 2824 synthetic organic fibers.

TABLE A.16. 1972 Production and Energy Consumption for Selected SIC 2824 Synthetic Organic Fibers (Battelle 1976)

\begin{tabular}{|c|c|c|c|}
\hline \multirow[b]{2}{*}{ Fiber } & \multirow{2}{*}{$\begin{array}{l}\text { Production } \\
\left(10^{9} \mathrm{1b}\right) \\
\end{array}$} & \multicolumn{2}{|c|}{ Energy Consumption } \\
\hline & & $\left(10^{12} \mathrm{Btu}\right)$ & $(B t u / 1 b)$ \\
\hline Polyester & 2327 & 46.2 & 19,900 \\
\hline Nylon & 1974 & 49.4 & 25,500 \\
\hline Acryl ic & 626 & 29.5 & 47,100 \\
\hline Polyolefin & 347 & 8.5 & 24,600 \\
\hline Other & 82 & 2.1 & 25,000 \\
\hline Total & 5356 & 135.7 & \\
\hline
\end{tabular}

The following sections describe the processes, heat-transfer operations, and waste heat streams associated with the production of polyester, nylon, and acrylic fibers.

Process Description

In general, fibers are made by reacting simple molecules (monomers) with identical or similar molecules to form long chains of repeating units (polymers). The polymers are spun into fibers, drawn, and wound. The following sections describe the processes used to produce polyester, nylon, and acrylic fibers.

\section{Polyester Fiber Production}

Polyester fiber is made from dimethyl terephthalate or terephthal ic acid and ethylene glycol. The polymerization reaction occurs at $260-300^{\circ} \mathrm{C}$ in a vacuum (Shreve and Brink 1977). The reaction products are methanol, ethylene glycol, and a long polymer chain. After melting, the polymer is spun and stretched into polyester fiber. The production process is shown in Figure A.13. 


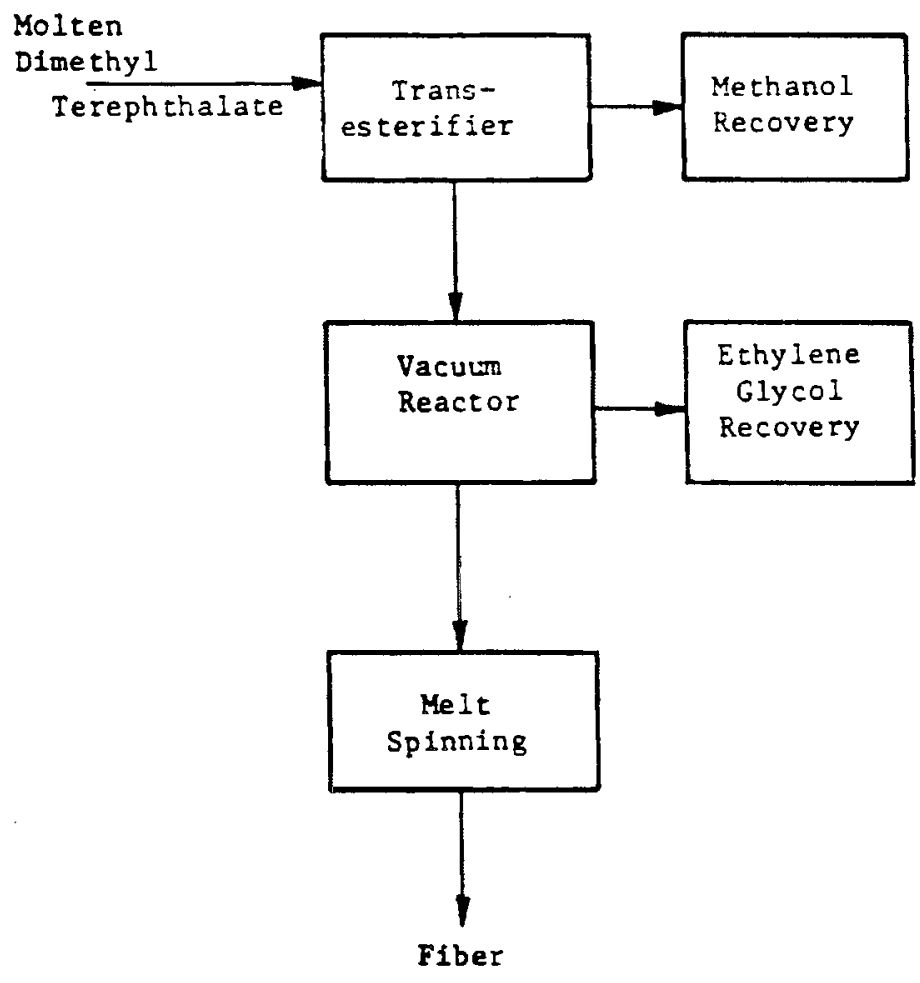

FIGURE A.13. Polyester Fiber Production (Battelle Columbus Laboratories 1976)

\section{Nylon Fiber Production}

There are two types of nylon produced in the United States: nylon 66 and nylon 6. In 1974, 70\% of the U.S. nylon production was nylon 66 and 30\% was nylon 6 (Shreve and Brink 1977).

Nylon 66 is produced by reacting hexamethylenediame and adipic acid to form "nylon salt." After water removal, the nylon salt is polymerized, solidified, and dried to form nylon chips. The chips are later melted, extruded, spun, and drawn into nylon fiber.

Nylon 6 is produced by the water-catalyzed polymerization of caprolactam (Figure A.14). The caprolactam and water are mixed and heated in a reactor to $500^{\circ} \mathrm{F}$. Heat from the exothermic reaction is removed by a heat-transfer fiuid such as Dowtherm (a trademark of the Dow Chemical Company). Unreacted monomer is removed and the polymer is cooled and dried, then later melted and spun into nylon fiber. 


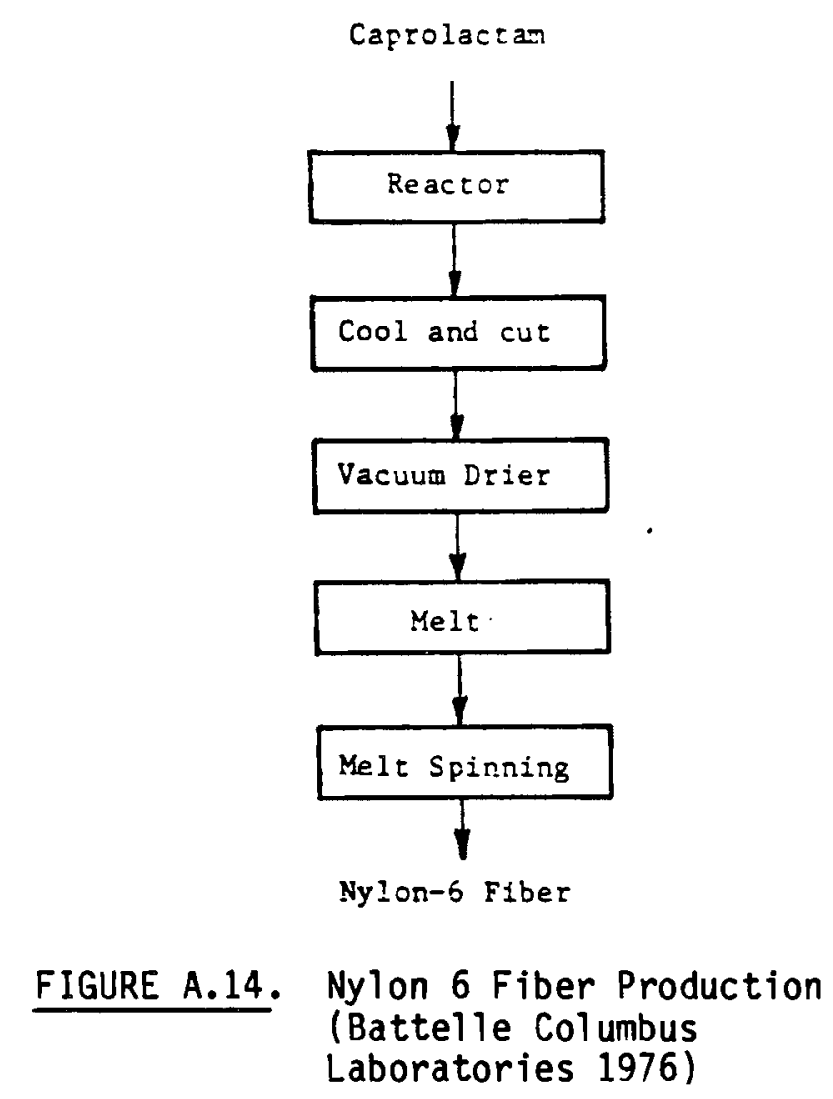

\section{Acryl ic Fiber Production}

Acrylic fiber is produced by the polymerization of acrylonitrile in an aqueous system. Unreacted monomer is removed by water washing. The polymer is then dried and ground. Since it decomposes at its melting point, the polymer is dissolved in dimethylformamide and either dry spun or wet spun into acrylic fiber. This production process is shown in Figure A.15.

Heat-Transfer Operations

Polymerization reactions are exothermic, but may require high temperatures to initiate. Therefore, both heating and cooling of reactors is necessary. This is usually accomplished with a fluid such as Dowtherm (a trademark of the Dow Chemical Company), which is preheated with fuel.

Steam is used for melting, evaporation, distillation, and various other unit operations. Cooling water and air are also used. Large amounts of electricity are required for the spinning processes. 


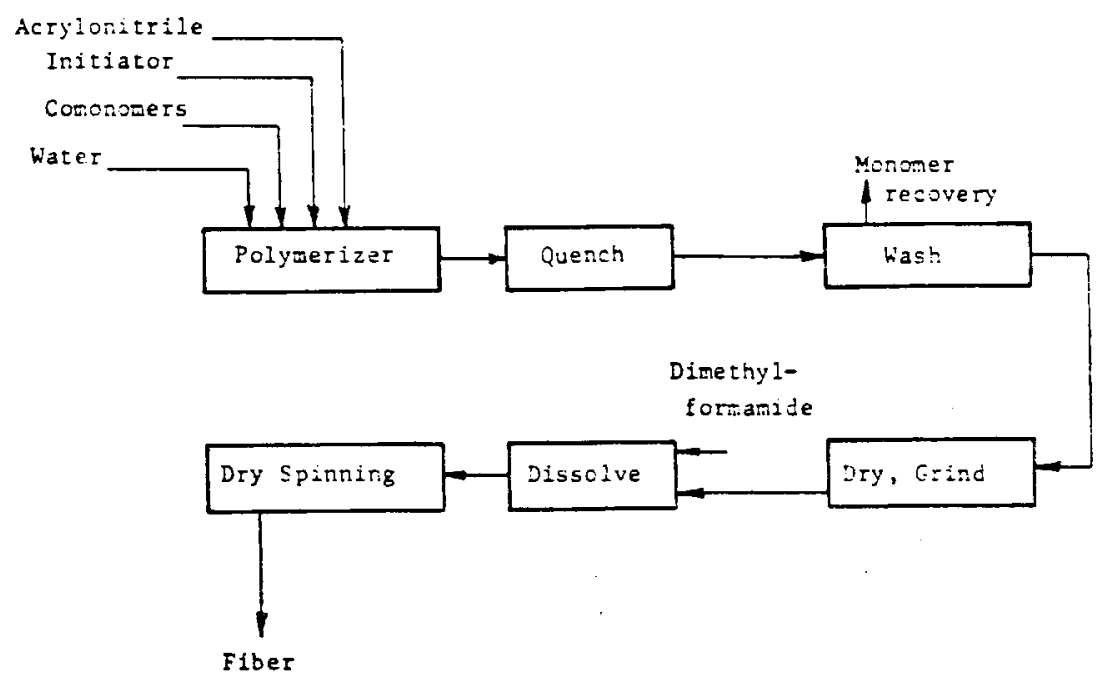

FIGURE A.15. Acrylic Fiber Production (Battelle Columbus Laboratories 1976)

Table A.17 shows the 1971 and 1973 average energy consumption for the production of six synthetic fibers: rayon, acetate, polyester, nylon, acrylicmodacrylic, and olefin. The energy consumption figures differ by a factor of three between the most-energy-intensive fiber (acetate) and the least-energyintensive fiber (polyester) (Sittig 1977).

TABLE A.17. Energy Consumption in Synthetic Fiber Production (Federal Energy Administration 1974)

Energy Consumption

\begin{tabular}{|c|c|c|}
\hline \multirow[b]{2}{*}{ Fiber } & \multicolumn{2}{|c|}{$\left(10^{3} \mathrm{Btu} / 1 \mathrm{~b}\right)$} \\
\hline & 1971 & 1973 \\
\hline Rayon & 61.9 & 50.4 \\
\hline Acetate & 67.2 & 63.0 \\
\hline Polyester & 19.9 & 17.3 \\
\hline Nylon & 25.0 & 20.6 \\
\hline Acryl ic-modacryl ic & 47.1 & 43.4 \\
\hline Olefin & 24.6 & 19.0 \\
\hline
\end{tabular}


Table A.18 shows the 1975 energy consumption by energy source for SIC 2824. Fuel oil represented 42 percent of the total consumption of purchased fuel, coal represented 35 percent, and natural gas represented 19 percent.

TABLE A.18. 1975 Energy Consumption for SIC 2824

(U.S. Department of Commerce 1975)

\section{Energy Source}

Fuel 0 il

Coal

Natural Gas

Other (a)

Purchased Fuel (Total)

Purchased Electricity(b)

Purchased Fuel and Electric Energy

(Grand Total)
Consumption (1012 Btu)

43.15

35.91

19.76

$\frac{4.59}{103.41}$

21.16

124.57

(a) "Other" includes "fuels not specified by kind.

(b) Purchased electricity converted at $3413 \mathrm{Btu} / \mathrm{kWh}$; self-generated electricity is al ready included in the individual fuel consumptions above to avoid double counting.

Heat Transfer in Polyester Fiber Production

Electricity accounts for 55 percent of the energy required for polyester fiber production (Palmer et al. 1980). Most of the electricity is used in the fiber-spinning operation and the air conditioning required to solidify the fiber.

Steam is used for melting and for distillation. The polymerization reactors are usually heated with Dowtherm. Cooling water and air are used to remove excess heat. 
Heat Transfer in Nylon Fiber Production

Electricity is also the major energy input to the nylon fiber production process. It is used to run the motors in the extrusion and spinning/winding operations and also for air conditioning (Palmer et al. 1980).

Steam is used in the evaporation and concentration of the nylon salt. The polymerization reactor is heated with Dowtherm. Since the melting point of nylon-6,6 is above $500^{\circ} \mathrm{F}$, high temperatures are required in the process (Palmer et al. 1980). The required temperature range for nylon 66 is $290-295^{\circ} \mathrm{C}$; for nylon 6 it is $280-285^{\circ} \mathrm{C}$. The Dowtherm is heated in a boiler using natural gas for fuel (United Technologies Corporation 1980).

Waste Heat Streams

The only major waste heat streams from polyester fiber production are cooling water and air. The only major waste heat stream from nylon 66 fiber production is cooling water (Palmer et al. 1980). Cooling water waste heat streams for two typical synthetic plants are shown in Table A.19. Cooling air for fiber-spinning operations is shown for polyester production.

TABLE A.19. Waste Heat Streams from Synthetic Fiber Plants (Palmer et al. 1980)

\begin{tabular}{|c|c|c|c|c|}
\hline Plant Type & $\begin{array}{l}\text { Plant Size } \\
\text { (tons/day) }\end{array}$ & $\begin{array}{c}\text { Waste Stream } \\
\text { Type }\end{array}$ & $\begin{array}{c}\text { Temperature } \\
\left({ }^{\circ} \mathrm{F}\right)\end{array}$ & $\begin{array}{l}\text { Flow Rate } \\
(1 \mathrm{~b} / \mathrm{hr}) \\
\end{array}$ \\
\hline \multirow[t]{2}{*}{ Polyester } & 250 & Cooling water & 100 to 120 & $10 \times 10^{6}$ \\
\hline & & Cooling air & 90 to 110 & $750 \times 10^{3}$ \\
\hline Nylon 66 & 150 & Cooling water & 100 to 120 & $2.5 \times 10^{6}$ \\
\hline
\end{tabular}

CYCLIC CRUDES AND INTERMEDIATES - SIC 2865

This SIC code contains cyclic (coal tar) crudes and cyclic intermediates, dyes, and organic pigments. Of the 50 chemicals with the largest 1975 sales volumes in the U.S., the following 10 are included in SIC 2865 (Sittig 1977): 
Benzene

Toluene

Xylene

Ethylbenzene

Styrene
Terephthal ic Acid (and Dimethyl

Terephthalate)

$\mathrm{p}-\mathrm{Xy}$ lene

Cumene

Cyclohexane

Phenols

Some of these chemicals are classified under more than one SIC code, depending upon the feedstock used in the production process. For example, 90 percent of the U.S. production of benzene is classified under SIC 2911 (petroleum refining), and only 10 percent is included in SIC 2865 (Bodine and Vitullo 1980).

Thousands of chemicals are included in SIC 2865. Many of them are produced by two or more processes with different feedstocks and energy requirements. In 1972 the total production of SIC 2865 chemicals was 61 billion pounds; the total energy consumption for 1972 was 192 trillion Btu (Battelle 1976).

Table A.20 shows the 1972 production levels of the top ten cyclic intermediates. In 1972 the U.S. produced almost 35 billion pounds of cyclic

TABLE A.20. 1972 Production of Important Cyclic Intermediates (U.S. Tariff Commission 1975)

\begin{tabular}{lr}
\multicolumn{1}{c}{ Chemical } & $10^{3}$ Pounds \\
Styrene & $5,940,729$ \\
Ethylbenzene & $5,675,900$ \\
Cyclohexane & $2,298,394$ \\
Cumene & $2,292,949$ \\
p-Xyl ene & $2,207,556$ \\
Phenol & $2,096,125$ \\
Phthal ic Anhydride & 952,978 \\
o-Xyl ene & 831,782 \\
Nitrobenzene & 551,169 \\
Dodecylbenzenes (a) & 524,009 \\
& \\
\hline (a) Includes branched- and straight-chain \\
dodecylbenzene and tridecylbenzene.
\end{tabular}


intermediates (Shreve and Brink 1977), which accounts for 57 percent of the total production of SIC 2865 chemicals.

The following sections describe the processes, heat-transfer operations, and waste heat streams associated with the production of two representative SIC 2865 chemicals: styrene and phenol.

\section{Process Description}

The cyclic intermediates are the raw materials used to produce dyes, medicinals, plastics, rubbers, and synthetic fibers. The main types of chemical conversions used to produce cyclic intermediates are nitration, amination by reduction, amination by ammonolysis, halogenation, sulfonation, hydrolysis, oxidation, and condensation and addition reactions (Friedel-Crafts) (Shreve and Brink 1977). The following sections describe the processes used to produce styrene and phenol.

\section{Styrene Production}

Styrene is produced by the catalytic dehydrogenation of ethylbenzene. The ethylbenzene is produced by the catalytic alkylation of benzene with ethylene. It is common practice in the literature to discuss these two processes together.

Ethylbenzene can be produced by two different processes: one uses an aluminum chloride catalyst and the other uses a boron trifluoride catalyst. Most ethylbenzene is produced using the aluminum chloride catalyst. The alkylation reaction conditions are $200-205^{\circ} \mathrm{F}$ and 5 psig (United Technologies Corporation 1980). The reactor effluent contains ethylbenzene, catalyst, unconverted benzene, and by-product diethylbenzene. The ethylbenzene product is purified in a series of three distillation columns.

Styrene production requires an iron oxide catalyst and a large quantity of steam. The dehydrogenation of ethylbenzene occurs at a temperature of 1050$1100^{\circ} \mathrm{F}$ and a pressure of $10 \mathrm{psig}$ or less (United Technologies Corporation 1980). The reactor effluent contains styrene, unconverted ethylbenzene, and small quantities of by-products. The styrene product is purified in a series of distillation columns. 


\section{Phenol Production}

Phenol can be produced by four different processes: oxidation of cumene, the Raschig process, sulfonation of benzene, and oxidation of toluene. The cumene oxidation process was used to produce 87 percent of the 1973 phenol output. The toluene oxidation process is seldom used (Bodine and vitullo 1980).

In the cumene oxidation process, cumene is oxidized with air to produce phenol and by-product acetone. The major unit operations employed in the process are catalytic reaction, distillation, vacuum evaporation, gas compression, and pumping. The major energy consumption occurs in the distillation column reboilers. All process heat requirements are provided by steam (Palmer et a1. 1980).

Heat-Transfer Operations

Table A.21 shows the 1972 conversion energy requirements for manufacturing selected SIC 2865 chemicals. The energy requirements in this table are categorized as lb steam per pound of product, kWh electricity per pound of product, and Btu other energy sources per pound of product. Table A.22 summarizes the 1972 total conversion energy requirements for manufacturing the same SIC 2865 chemicals.

Table A.23 shows the 1975 energy consumption by energy source for SIC 2865. Natural gas represented 60 percent of the total energy consumption, fuel oil represented 22 percent, purchased electricity represented 10 percent, and coal represented 8 percent.

Heat Transfer in Styrene Production

Large amounts of steam are required for the styrene production reaction and for the ethylbenzene and styrene distillation columns. Either natural gas or fuel oil is used as a boiler fuel. Steam and fuel requirements for ethylbenzene and styrene production plants are shown in Table A.24. In addition to the $0 i 1$ and gas fuels, over $200 \times 10^{6} \mathrm{Btu} / \mathrm{hr}$ of by-product hydrogen and waste oils are produced and consumed as fuel in a styrene production plant (Palmer et al. 1980). 
TABLE A.21. 1972 Conversion Energy Requirements for Manufacturing Selected SIC 2865 Chemicals (Battelle 1976)

\begin{tabular}{|c|c|c|c|c|c|c|}
\hline \multirow[b]{2}{*}{ Chemical } & \multirow[b]{2}{*}{ Process } & \multicolumn{3}{|c|}{$\begin{array}{l}\text { Conversion Energy } \\
\text { (per pound of product) }\end{array}$} & \multirow[b]{2}{*}{$\frac{\text { Total }}{\text { Net }}$} & \multirow[b]{2}{*}{$\frac{(B t u / 1 b)}{\text { Gross }}$} \\
\hline & & $\begin{array}{l}\text { Steam } \\
(1 \mathrm{~b})\end{array}$ & $\begin{array}{c}\text { Electricity } \\
(\mathrm{kWh})\end{array}$ & $\begin{array}{l}\text { Other } \\
\text { (Btu) }\end{array}$ & & \\
\hline Benzene & Shell sulfolane & -- & -- & -- & 620 & 680 \\
\hline Benzene & Detol & $0.044^{(c)}$ & 0.0245 & $(3870)$ & $(3740)$ & $(3560)$ \\
\hline Benzene & Pyrotol & $0.065^{(c)}$ & 0.0236 & $(10900)$ & $(10740)$ & $(10570)$ \\
\hline Bisphenol A & Union Carbide & $5.3^{(d)}$ & 0.23 & - & 7810 & 9410 \\
\hline Cyclohexane & $\begin{array}{l}\text { hydrogenation of } \\
\text { benzene-ARCO }\end{array}$ & nil & nil & nil & nil & nil \\
\hline $\begin{array}{l}\text { Dimethyl Terephthalate } \\
\text { (fiber grade) }\end{array}$ & $\begin{array}{l}\text { Dynamit Nobel from } \\
\text { p-xylene }\end{array}$ & $1.2^{(d)}$ & 0.23 & 1800 & 4170 & 5780 \\
\hline Phenol & cumene & $\begin{array}{l}3.28(e) \\
0.5\end{array}$ & 0.071 & 117 & 5860 & 6355 \\
\hline Styrene & Monsanto & $\begin{array}{l}1.35(c) \\
2.4\end{array}$ & 0.038 & 2160 & 7020 & 7290 \\
\hline P-xylene & Toray Ind.-UOP & $1.04(c)$ & 0.14 & -- & 1670 & 2650 \\
\hline
\end{tabular}

(a) Electricity equivalence: $3,413 \mathrm{Btu} / \mathrm{kWh}$.

(b) Electricity equivalence: $10,400 \mathrm{Btu} / \mathrm{kWh}$.

(c) Low-pressure steam: 1,150 Btu/1b.

(d) Medium-pressure steam: 1,325 Btu/1b.

(e) High-pressure steam: 1,500 Btu/1b. 
TABLE A.22. 1972 Total Conversion Energy Requirements for Manufacturing Selected SIC 2865 Chemicals (Battelle 1976)

\begin{tabular}{|c|c|c|c|c|c|c|}
\hline \multirow[b]{2}{*}{ Chemical } & \multirow[b]{2}{*}{ Process } & \multirow{2}{*}{$\frac{\operatorname{SEC}(a)}{\text { Net }}$} & \multirow{2}{*}{$\frac{(\mathrm{Btu} / 1 \mathrm{~b})}{\mathrm{Gross}}$} & \multirow{2}{*}{$\begin{array}{l}\text { Production (b) } \\
\text { (million 1b) }\end{array}$} & \multicolumn{2}{|c|}{$\begin{array}{c}\text { Total Conversion } \\
\text { Energy (billion Btu) }\end{array}$} \\
\hline & & & & & Net & Gross \\
\hline Benzene & Shell sulfolane & 620 & 680 & 8654 & 5365 & 5885 \\
\hline Bisphenol A & Union Carbide & 7810 & 9410 & 255.2 & 1993 & 2401 \\
\hline Cyclohexane & $\begin{array}{l}\text { hydrogenation of } \\
\text { benzene }\end{array}$ & nil & nil & 2298 & nil & nil \\
\hline $\begin{array}{l}\text { Dimethy } 1 \\
\text { Terephthal ate }\end{array}$ & Dynamit Nobel & 4170 & 5780 & 2167 & 9036 & 12525 \\
\hline Phenol & cumene & 5860 & 6355 & 2052 & 12025 & 13040 \\
\hline Styrene & Monsanto & 7020 & 7290 & 5940 & 41699 & 43303 \\
\hline$P$-xylene & Toray Ind.-UOP & 1670 & 2650 & 2208 & 3687 & 5851 \\
\hline Total & & -- & -- & 23574 & 73805 & 83005 \\
\hline $\begin{array}{l}\text { Average } \\
\text { Values }\end{array}$ & & 3131 & 3521 & -- & - & -- \\
\hline
\end{tabular}

(a) SEC: Specific Energy Consumption.

(b) Production statistics from "Synthetic Organic Chemicals, United States Production and Sales, 1972," United States Tariff Commission, Washington, D.C., 1974. 
TABLE A.23. 1975 Energy Consumption for SIC 2865

(U.S. Department of Commerce 1975)

\begin{tabular}{lrl}
\multicolumn{1}{c}{ Energy Source } & \multicolumn{1}{c}{ Consumption } \\
\hline Fuel 0il & $35.99 \times 10^{12} \mathrm{Btu}$ \\
Coal & $12.59 \times 10^{12} \mathrm{Btu}$ \\
Natural Gas & $96.25 \times 10^{12} \mathrm{Btu}$ \\
Purchased Electricity & $4.5 \times 10^{9} \mathrm{kWh}$
\end{tabular}

TABLE A.24. Steam and Fuel Requirements for Ethylbenzene and Styrene Plants (Palmer et al. 1980)

\begin{tabular}{|c|c|c|c|c|}
\hline Plant Type & $\begin{array}{l}\text { Plant Size } \\
\text { (tons/day) }\end{array}$ & $\begin{array}{l}\text { Steam } \\
\text { Pressure } \\
\text { (psig) } \\
\end{array}$ & $\begin{array}{c}\text { Steam } \\
\text { Flow Rate } \\
(1 \mathrm{~b} / \mathrm{hr}) \\
\end{array}$ & $\begin{array}{c}0 i 1 / \text { Gas } \\
\text { Consumption } \\
\left(10^{6} \text { Btu/hr) }\right. \\
\end{array}$ \\
\hline Ethylbenzene & 1700 & 600 & 220,000 & 140 \\
\hline Styrene & 1500 & 75 & 320,000 & 16 \\
\hline Styrene & 1500 & 30 & 190,000 & - \\
\hline
\end{tabular}

In ethylbenzene production, distillation accounts for about 15 percent of the total energy requirements. In styrene production, heating the reactants and the steam and distilling the reactor effluent account for over 90 percent of the total energy requirements (Latour et al. 1981).

The production of styrene is thermal-energy-intensive, with most of the energy supplied by steam. Natural gas is used to superheat the steam for the endothermic ethylbenzene dehydrogenation reaction. About 73 percent of the steam required for styrene production is used in the dehydrogenation reaction; the other 27 percent is used in distillation. About 94 percent of the steam required for ethylbenzene production is used in distillation (United Technologies Corporation 1980).

Table A.25 shows the cooling water requirements for ethylbenzene styrene production. About 67 percent of the cooling water is required for desuperheating and cooling the ethylbenzene dehydrogenation reactor product stream. 
TABLE A.25. Cooling Water Requirements for Ethylbenzene/Styrene Production (adapted from Latour et a1. 1982)

Source

Desuperheating and cooling ethylbenzene dehydrogenation reactor product stream

Condensing styrene distillation column overhead

Condensing ethylbenzene distillation column reflux

Cooling and scrubbing gaseous ethylbenzene alkylation reactor product stream

Cooling and decanting ethylbenzene alkylation reaction products

Total
Rate (gallons $\mathrm{H}_{2} \mathrm{O}$ / ton styrene)

79,000

19,400

14,400

3,600

1,350

117,750

About 16 percent is used to condense the styrene distillation column overhead, and about 12 percent is used to condense the ethylbenzene distillation column reflux.

\section{Heat Transfer in Phenol Production}

Large quantities of steam are required in the production of phenol by the cumene oxidation process, mostly to supply heat to the distillation column reboilers. For a typical 600-tons-per-day phenol plant, about 270,000 1b/hr of 200-psig steam and about $30,000 \mathrm{lb} / \mathrm{hr}$ of 600 -psig steam are required (Palmer et a1. 1980).

The cumene oxidation reaction is exothermic and careful control of the reactor temperature is mandatory. Cooling water is used to remove the excess heat of reaction.

Waste Heat Streams

In 1977 only about 5.5 percent of SIC 2865 purchased fuels and electricity was discharged as flue gas waste heat. Almost half of this energy was available at temperatures above $350^{\circ} \mathrm{F}$ (Latour et al. 1980). 
The following sections describe the sources and temperatures of waste heat streams associated with styrene and phenol production.

Waste Heat in Styrene Production

One reference states that styrene production results in about 88,000 cubic feet of flue gas at $400^{\circ} \mathrm{F}$ for each ton of styrene produced, which amounts to 620,000 Btu of waste heat per ton of styrene. The total waste heat discharged to cooling water and process water streams is 28.98 million Btu per ton of styrene (Latour et al. 1981). Thus, almost 50 times more waste heat is contained in the water streams than is contained in the flue gas.

Another reference states that the natural-gas-fired steam superheater in a styrene plant emits $500^{\circ} \mathrm{F}$ flue gas, representing $720,000 \mathrm{Btu}$ of waste heat per ton of styrene produced (United Technologies Corporation 1980).

Table A.26 shows typical waste heat streams discharged from ethylbenzene and styrene plants, according to a third reference.

TABLE A.26. Typical Waste Heat Streams in Ethylbenzene/Styrene Plants (Palmer et a1. 1980)

\begin{tabular}{|c|c|c|c|c|}
\hline Plant Type & $\begin{array}{l}\text { Plant Size } \\
\text { (tons/day) }\end{array}$ & Type & $\begin{array}{r}\text { Waste } \\
\text { Flow }(1 \mathrm{~b} / \mathrm{hr}) \\
\end{array}$ & $\frac{a m}{\text { lemp. (oF) }}$ \\
\hline Ethylbenzene & 1700 & $\begin{array}{l}\text { Heat Recovery } \\
\text { Steam }\end{array}$ & 198,000 & 240 \\
\hline Ethylbenzene & 1700 & $\begin{array}{l}\text { Heat Recovery } \\
\text { Steam }\end{array}$ & 124,000 & 360 \\
\hline Ethylbenzene & 1700 & Cooling Water & 245,000 & $100-120$ \\
\hline Ethylbenzene & 1700 & Flue Gas & 177,000 & 450 \\
\hline Styrene & 1500 & Cooling Water & $8,000,000$ & $100-120$ \\
\hline Styrene & 1500 & Flue Gas & 300,000 & 400 \\
\hline Styrene & 1500 & Air & 660,000 & $150-240$ \\
\hline
\end{tabular}




\section{Waste Heat in Phenol Production}

The only major source of waste heat from a phenol production plant is cool ing water. A 600-tons-per-day phenol plant would discharge 17,000,000 $1 \mathrm{~b} / \mathrm{hr}$ of $100-120^{\circ} \mathrm{F}$ cooling water (Palmer et al. 1980).

\section{ALIPHATIC ORGANIC CHEMICALS - SIC 2869}

This SIC code contains thousands of miscellaneous industrial organic chemicals that are not classified elsewhere. All but about 100 of these are produced in relatively small quantities. Many are produced by more than one process using different feedstocks and having different energy requirements. Of the 50 chemicals with the largest 1975 sales volumes in the U.S., the following 18 are included in SIC 2869 (Sittig 1977):

$\begin{array}{ll}\text { Ethylene } & \text { Acetic acid } \\ \text { Propylene } & \text { Acetone } \\ \text { Ethylene dichloride } & \text { Isopropyl alcohol } \\ \text { Methanol } & \text { Propylene oxide } \\ \text { Formaldehyde } & \text { Acetic anhydride } \\ \text { Vinyl chloride } & \text { Adipic acid } \\ \text { Ethylene oxide } & \text { Ethanol } \\ \text { Ethylene glycol } & \text { Acrylonitrile } \\ \text { Butadiene } & \text { Vinyl acetate }\end{array}$

Table A.27 shows the 1973 production and energy use statistics for selected SIC 2869 chemicals. Ethylene accounted for 29 percent of the production and 80 percent of the energy use. Ethylene is used exclusively as a building block and is the primary feedstock for over 40 percent of all petrochemical products (Tarrillion 1982). The following sections describe the process, heat-transfer operations, and waste heat streams associated with ethylene production. 
TABLE A.27. 1973 Production and Energy Use Statistics for Selected SIC 2869 Chemicals (International Research and Technology Corporation 1974)

\begin{tabular}{|c|c|c|}
\hline Chemical & $\begin{array}{l}\text { Production } \\
\left(10^{6} \text { tons }\right) \\
\end{array}$ & $\begin{array}{l}\text { Energy Use } \\
\left(10^{12} \text { Btu }\right) \\
\end{array}$ \\
\hline Ethylene & 11.0 & $1,460.26$ \\
\hline Ethylene dichloride & 3.96 & 4.17 \\
\hline Propylene & 4.0 & 103.76 \\
\hline Methanol & 3.52 & 124.69 \\
\hline Formal dehyde & 3.07 & 22.83 \\
\hline Ethylene oxide & 1.95 & 29.01 \\
\hline Ethylene glycol & 1.83 & - \\
\hline Acetone & 0.98 & 5.70 \\
\hline Acetal dehyde & 0.31 & 6.17 \\
\hline Acetic Anhydride & 0.79 & 12.88 \\
\hline Adipic acid & 0.72 & 19.42 \\
\hline Isopropanol & 0.90 & 11.79 \\
\hline Urea & 3.97 & 16.90 \\
\hline Ethyl alcohol & 0.97 & 11.42 \\
\hline Total & 37.97 & $1,829.00$ \\
\hline
\end{tabular}

Process Description

The feedstock for ethylene production can be ethane, propane, or naphtha. Figure A.16 is a flow diagram of the ethylene production process. The feedstock is cracked in a pyrolysis furnace, with steam injection provided to reduce coking (fouling of the furnace by carbon deposits). The hot furnace off gas is quenched with condensate, then compressed and distilled. The distillation products include ethylene, fuel oil, hydrogen, methane, propylene, butane derivatives, and gasoline. Ethane recovered from the distillation column is recycled to the pyrolysis furnace.

Heat-Transfer Operations

The high-temperature cracking reaction and the low-temperature distillation (which requires refrigeration) make this an energy-intensive process. The energy requirement, according to one source, is $20 \times 10^{6}$ Btu per ton of 


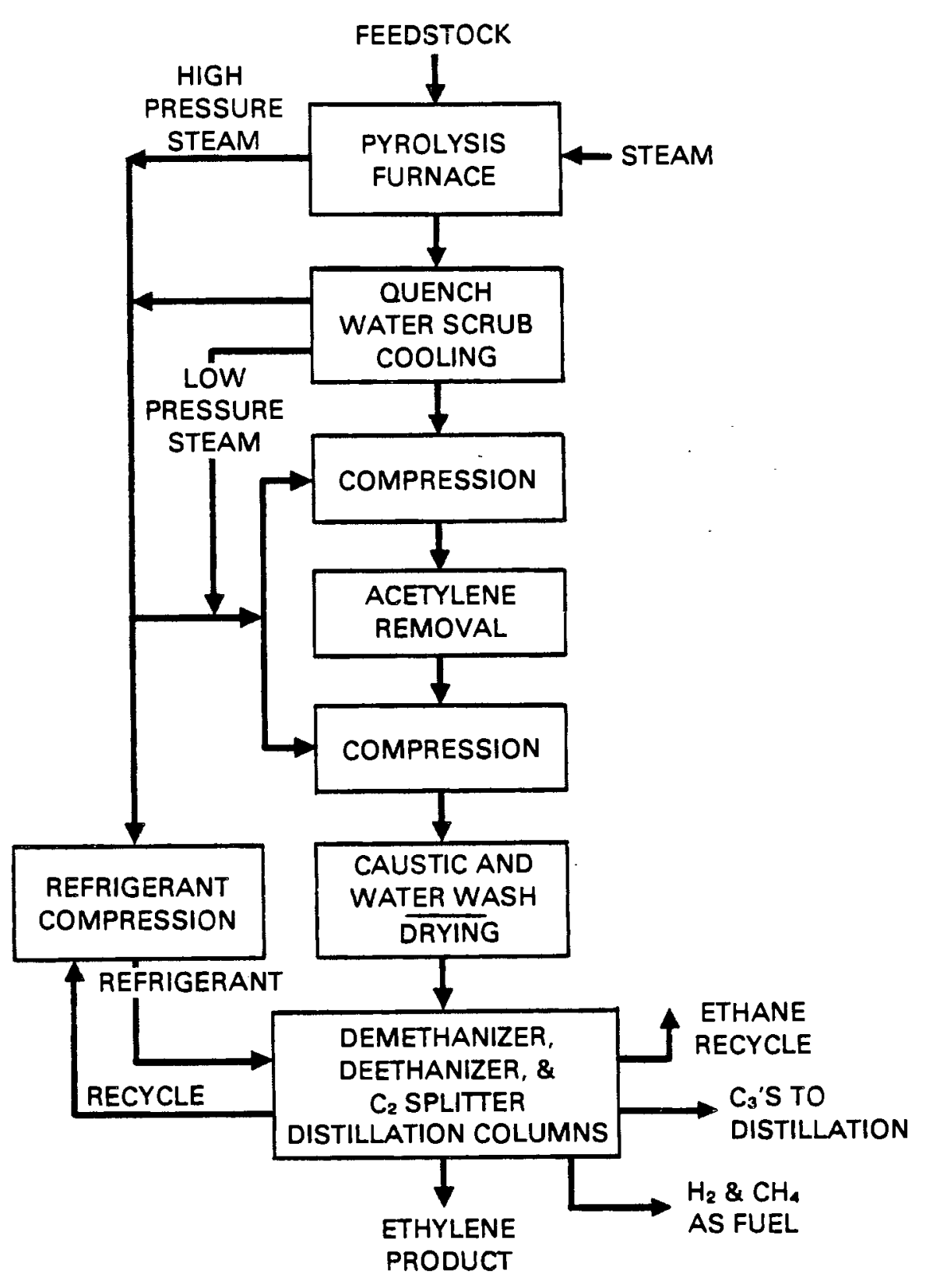

FIGURE A.16. Ethylene Process Flow Diagram

ethylene produced (Bodine and Vitullo 1980). Another reference states that the energy requirement is $32 \times 10^{6} \mathrm{Btu} /$ ton of ethylene (Palmer et al. 1980).

Table A.28 shows the 1971 and 1974 fuel and petroleum feedstock consumption for ethylene production. In each of the two years, natural gas accounted for 90 percent of the fuel consumption. Another reference shows that natural 
TABLE A.28. Fuel and Petroleum Feedstock Consumption for Ethylene Production (International Research and Technology Corporation 1974)

\begin{tabular}{|c|c|c|}
\hline & $\begin{array}{l}1971 \text { Data } \\
\left(10^{12} \mathrm{Btu}\right) \\
\end{array}$ & $\begin{array}{l}1974 \text { Data } \\
\left(10^{12} \text { Btu) }\right. \\
\end{array}$ \\
\hline Petroleum Feedstock & 842.65 & $1,151.53$ \\
\hline Petroleum Fuel & 4.40 & 5.68 \\
\hline Coal & 12.19 & 16.36 \\
\hline Natural Gas & 280.55 & 366.61 \\
\hline Other Fuels & 4.74 & 6.29 \\
\hline Purchased Electricity & 10.28 & 13.38 \\
\hline Total & $1,154.82$ & $1,559.85$ \\
\hline
\end{tabular}

gas represented about 75 percent of the total 1975 energy consumption for all of SIC 2869 (U.S. Department of Commerce 1978).

The endothermic cracking reaction occurs at $1500-1650^{\circ} \mathrm{F}$ in the pyrolysis furnace. Quenching the hot furnace off gas with condensate results in the generation of 400-2000 psig steam (Bodine and Vitullo 1980).

The pyrolysis furnace has a thermal efficiency greater than 85 percent. Using the fuel oil and gaseous by-products of the cracking reaction as fuels, the furnace heats the feedstock/steam mixture to the cracking temperature, superheats the steam, and heats the boiler feedwater (Palmer et al. 1980).

Waste-heat boilers are used to recover heat from the hot reaction products. Steam produced in the waste-heat boilers is injected into the pyrolys is furnace and is also used to provide process heat to the distillation column reboilers (Palmer et al. 1980). About two-thirds of the total plant steam requirement is generated in waste-heat boilers (United Technologies Corporation 1980).

Large amounts of cooling water are used to cool reaction products between compression stages. Cooling water is also used in the quench tower and to cool 
final product streams. As much as $38 \times 10^{6} \mathrm{lb} / \mathrm{hr}$ of cooling water at temperatures of 100 to $120^{\circ} \mathrm{F}$ may be discharged from a plant producing 1510 tons ethylene per day (Palmer et al. 1980). Most of the cooling water is used for between-stage compression.

Waste Heat Streams

Table A.29 shows the sources, temperatures, and quantities of waste heat from the ethylene production process. Stack gases from the pyrolysis unit, which contain $930 \mathrm{Btu} / \mathrm{lb}$, are available at $400^{\circ} \mathrm{F}$.

Table A.30 shows the sources, temperatures, and flow rates of waste heat identified by another literature reference (Palmer et al. 1980). This table shows $44,0001 \mathrm{~b} / \mathrm{hr}$ of 35 -psig steam being available, as well as $2.5 \times 10^{6} 1 \mathrm{~b} / \mathrm{hr}$ of flue gas at $350^{\circ} \mathrm{F}$. There is a large amount of cooling water, but it contains very low-grade heat that is not usable.

TABLE A.29. Sources, Temperatures, and Quantities of Waste Heat from Ethylene Production (Reding and Shepherd 1975)

\section{Source}

Radiation and Convection from Pyrolysis Furnace

Stack Gases from Pyrolysis Furnace

Water from Quenching, Scrubbing, and Cooling Hot

Reaction Products from Pyrolys is Furnace

Radiation and Convection from Compression of Cool Reaction Products

Condensate from Compression of Cool Reaction Products

Compressed Reaction Products

Radiation and Convection from Refrigerant Compression

Condensate from Refrigerant Compression

Refrigerant

Conduction from Distillation Columns
Temperature Quantity

( $\left.{ }^{\circ} \mathrm{F}\right)$

(Btu/1b)

150

400

930

150

100

220

1400

220

750

$-$

220

1500

150

1000

250 
TABLE A.30. Sources, Temperatures, and Flow Rates of Waste Heat from Ethylene Production (Palmer et al. 1980)

\begin{tabular}{lcrr}
\multicolumn{1}{c}{ Source } & & $\begin{array}{c}\text { Temperature } \\
\left({ }^{\circ} \mathrm{F}\right)\end{array}$ & \multicolumn{1}{c}{$\begin{array}{c}\text { Flow Rate } \\
(1 \mathrm{~b} / \mathrm{hr})\end{array}$} \\
\cline { 1 - 1 } $\begin{array}{l}35 \text { psig Steam from Waste- } \\
\text { Heat Boilers }\end{array}$ & 260 & & 44,000 \\
Flue Gas & 350 & \\
Cooling Water & $100-120$ & $38,000,000$
\end{tabular}

Table A.31 shows the sources and quantities of waste heat from a third reference (Latour and Menningmann 1981). The only major gas waste heat stream is the $400^{\circ} \mathrm{F}$ pyrolysis furnace stack gas. The total heat rejected by process waste water and non-contact cooling water amounts to $9.5 \times 10^{6} \mathrm{Btu} /$ ton of ethylene produced.

Ethylene production is a relatively clean process if ethane or propane is used as the feedstock. Both the feedstock and the primary fuel (natural gas) are very low in sulfur. The process has no major uncontrollable air, water, or sol id waste emissions (Arthur D. Little, Inc. 1976).

TABLE A.31. Sources and Quantities of Waste Heat from Ethylene Production (Latour and Menningmann 1981)

\begin{tabular}{|c|c|c|c|}
\hline Source & $\begin{array}{c}\text { Quantity/Ton } \\
\text { Ethylene } \\
\end{array}$ & $\begin{array}{c}\text { Temperature } \\
\left({ }^{\circ} \mathrm{F}\right)\end{array}$ & $\begin{array}{r}\text { Btu/Ton } \\
\text { Ethylene } \\
\end{array}$ \\
\hline \multicolumn{4}{|l|}{ Gas } \\
\hline $\begin{array}{l}\text { Pyrolysis Furnace } \\
\text { Stack Gas }\end{array}$ & $233,000 \mathrm{ft}^{3}$ & 400 & $1,860,000$ \\
\hline \multicolumn{4}{|l|}{ Water } \\
\hline Compressor Blowdown & 5,200 gal & -- & $5,800,000$ \\
\hline $\begin{array}{l}\text { Refrigerant Cooling } \\
\text { Water }\end{array}$ & 3,500 gal & -- & $2,000,000$ \\
\hline $\begin{array}{l}\text { Pyrolysis Gas Cooling } \\
\text { Water }\end{array}$ & $1,340 \mathrm{gal}$ & -- & $1,500,000$ \\
\hline Quench Water Blowdown & 350 gal & -- & 200,000 \\
\hline
\end{tabular}


NITROGENOUS FERTILIZERS - SIC 2873

The nitrogenous fertilizer industry encompasses the manufacture of synthetic ammonia, nitric acid, ammonium compounds and urea. This product category is of particular interest because it is one of the top producers in terms of annual tonnage in the chemical industry and it is one of the major users of natural gas as a feedstock material. Ammonia dominates the production of industrial fertilizers. Anmonia is the basic raw material for most nitrogenous fertilizers, including urea, ammonium nitrate, and ammonium phosphate. The manufacturing process, major heat-transfer operations, and waste heat streams associated with the production of ammonia are described below.

Process Description

Ammonia is made primarily by the steam reforming of natural gas, al though other sources of hydrogen such as LPG, naphtha, heavy fuel oil, coal, electrolytic hydrogen, or by-product hydrogen could be used (Arthur D. Little, Inc. 1976). Atmospheric air is used as the source of nitrogen.

Figure A.17 shows the major steps in ammonia manufacture. The process consists of four basic steps: 1) fuel reforming, 2) shift conversion of carbon monoxide, 3) gas purification, and 4) ammonia synthesis.

Fuel reforming converts the fuel and steam to carbon monoxide and hydrogen as follows:

$$
\mathrm{CH}_{4}+\mathrm{H}_{2} \mathrm{O}+3 \mathrm{H}_{2}+\mathrm{CO}
$$

The desulfurized natural gas and steam are fed to the primary reforming furnace. Natural gas is converted to hydrogen and carbon monoxide in the presence of a nickel catalyst inside of externally heated tubes. The product stream exits the primary reformer at about $1500^{\circ} \mathrm{F}$ and 460 psia (sittig 1977). Stoichiometric quantities of compressed air are fed to the secondary reformer along with the product stream from the primary reformer. The residual methane in the product steam is converted as the gases pass over a nickel catalyst in a packed bed. The product gases exit the secondary reformer at about $1800^{\circ} \mathrm{F}$ (Sittig 1977). 


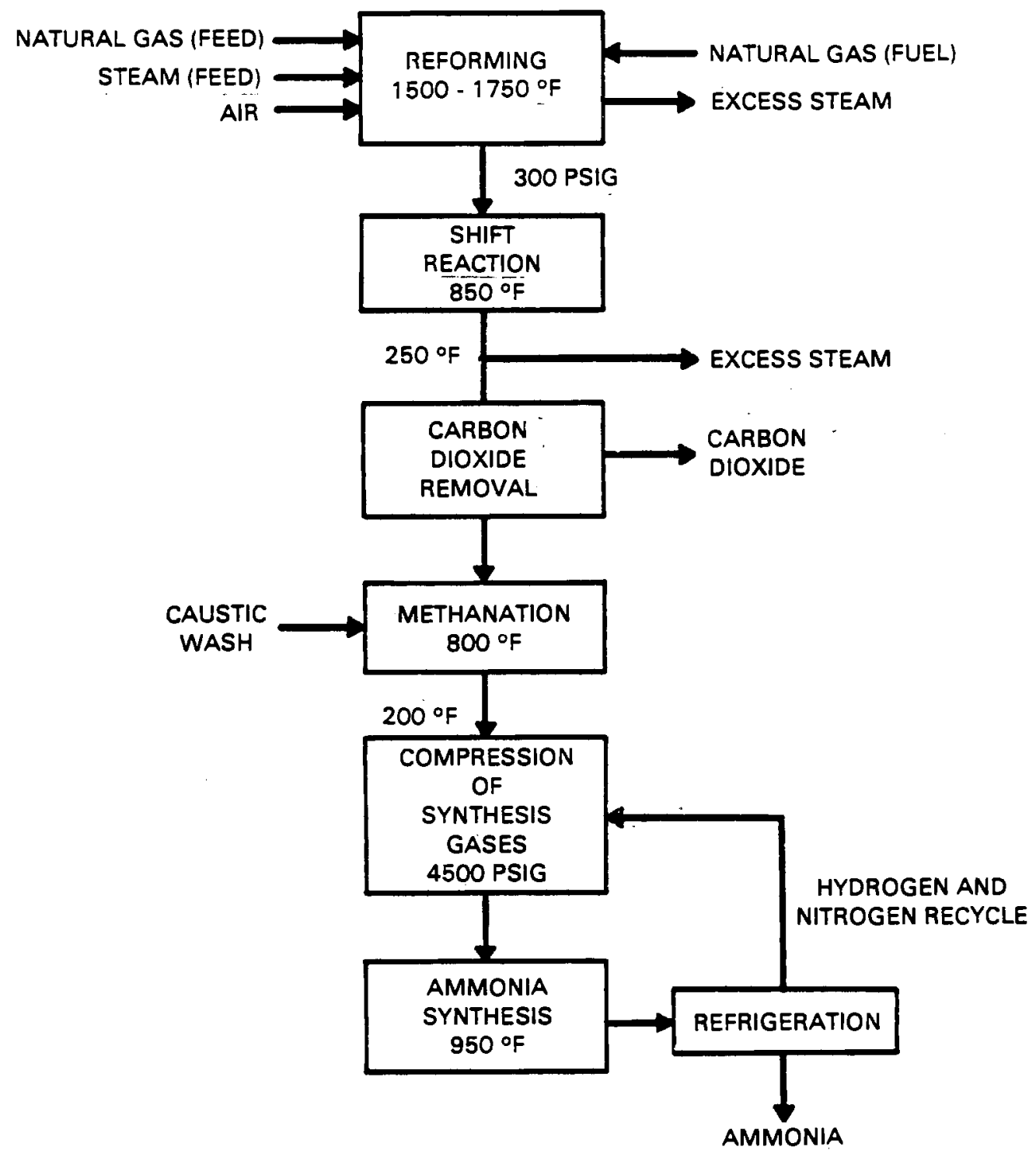

FIGURE A.17. Ammonia Production Process (Batte11e 1976)

The next step is a shift conversion that converts carbon monoxide and water to carbon dioxide and hydrogen as follows:

$$
\mathrm{CO}+\mathrm{H}_{2} \mathrm{O} \rightarrow \mathrm{CO}_{2}+\mathrm{H}_{2}
$$

This occurs in a converter containing a catalyst.

Next, the gases are purified by removing the carbon dioxide, residual carbon monoxide, and the catalyst poisons. The carbon dioxide is removed by 
stripping with one of various types of solvents. Carbon monoxide is typically removed using methanation, whereby the residual carbon monoxide is converted to methane according to the following reaction:

$$
\mathrm{CO}+3 \mathrm{H}_{2}+\mathrm{CH}_{4}+\mathrm{H}_{2} \mathrm{O}
$$

Ammonia synthesis takes place in the presence of a catalyst at elevated pressure and temperature in a packed bed. A multipass arrangement is used to achieve complete conversion. The synthesis reaction is as follows:

$$
\mathrm{N}_{2}+3 \mathrm{H}_{2}+2 \mathrm{NH}_{3}
$$

Ammonia plants range in size from 50 to 2000 tons/day, with the average plant producing about 220 tons/day (Palmer 1980). Heat-Transfer Operations

The major heat-transfer operations in ammonia manufacture are 1 isted in Table A.32.

The major energy users in an ammonia plant are the reforming furnaces and the compressors. A typical plant uses 155,000 Btu of electricity and $33 \times$ $10^{6}$ Btu of natural gas or fuel oil per ton of ammonia produced. of the fue 1 used, $62 \%\left(20.5 \times 10^{6} \mathrm{Btu} /\right.$ ton) is feedstock (Latour 1981). Ammonia plants are designed such that the entire fuel input to the process is to the primary reforming furnace. Fuel burned in the primary reformer averages 12.5 million Btu per ton of ammonia produced (Palmer 1980). About $3.5 \times 10^{6}$ Btu of purge

TABLE A.32. Major Heat-Transfer Operations in Ammonia Manufacture

Operation

Reforming

Waste Heat Recovery

Compression

Compressor Cooling
Description

Fired-heater with combustion gases on shell side and natural gas, steam and the catalyst on the tube side.

Waste-heat boilers are used to generate steam from reforming furnace exhausts.

The steam generated by the waste-heat boilers is used primarily for operating the compressors prior to the synthes is reaction.

Compressors are water cooled. 
gas from the synthesizer is recycled and burned in the primary reformer (Latour 1981). Waste heat is then recovered from the flue gases from the primary reforming furnace and from the product gases from the secondary reformer to generate steam in a waste-heat boiler. Plants that have package boilers generally use them only during plant startup unless steam is needed for the manufacture of derivatives of ammonia (Arthur D. Little, Inc. 1976). Little or no steam beyond that produced in the waste-heat boilers is needed at steadystate conditions.

Waste Heat Streams

Sources of waste heat in ammonia production include flue gases from the primary and secondary reformers, dryers and coolers, and combustion equipment; non-contact cooling water; and process waters from strippers. Cool ing water in ammonia plants is used to cool the product stream: 1) after secondary reforming, 2) between high- and low-temperature shift conversion, 3) in compressors, and 4$)$ in the ammonia synthesis step. Table A.33 lists the waste heat discharged in liquid streams. Table A.34 shows the waste heat sources and quantities estimated for a 1200 ton/day ammonia plant.

TABLE A.33. Heat Rejected in Wastewater Effluents from Ammonia Plants (Latour 1981)

\begin{tabular}{|c|c|c|}
\hline Source & $\begin{array}{c}\text { Temperature } \\
\left({ }^{\circ} \mathrm{F}\right)\end{array}$ & $\begin{array}{l}\text { Quantity (b) } \\
\text { (Btu/ton) }\end{array}$ \\
\hline Boiler Blowdown & 220 & 27,000 \\
\hline Cooling Tower Blowdown & 20 & 175,000 \\
\hline Stripped Process Condensate & 70 & 175,000 \\
\hline
\end{tabular}

(a) Temperature above ambient.

(b) Waste heat discharge per ton of product.

TABLE A.34. Waste Heat Sources and Quantities in Ammonia Production--1200 Ton/Day Plant (Palmer 1980)

\begin{tabular}{|c|c|c|}
\hline Source & $\begin{array}{c}\text { Temperature } \\
\left({ }^{\circ} \mathrm{F}\right)\end{array}$ & $\begin{array}{l}\text { Quantity } \\
(1 \mathrm{~b} / \mathrm{hr})\end{array}$ \\
\hline Reformer Flue Gas & 400 & 500,000 \\
\hline Compressor Cooling & $100-120$ & $24 \times 10^{6}$ \\
\hline
\end{tabular}




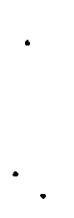


APPENDIX B

PETROLEUM REFINING AND RELATED INDUSTRIES - SIC 29 


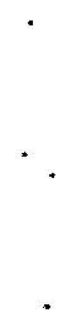




\section{APPENDIX B}

PETROLEUM REFINING AND RELATED INDUSTRIES - SIC 29

This industry classification includes establishments that are primarily engaged in petroleum refining, manufacturing paving and roofing materials, and compounding lubricating oils and greases from purchased materials. Table B.1 shows the 4-digit industrial groups in SIC 29. Since petroleum refining accounts for about 95 percent of the total energy consumption of SIC 29 , it is the only 4-digit industrial group that will be discussed in the following sections.

The petroleum refining industry ranks second in terms of industrial energy use. It consumes about 24 percent of the total energy used by the industrial manufacturing sector. The petroleum refining industry is characterized by yeararound, 24-hour-per-day operations, 7 days per week, except for planned and emergency shutdowns.

The petroleum refining industry is extremely energy-intensive. However, as the first supplier of refined fuels to the rest of the country, it has access to by-product fuels not available elsewhere, such as refinery gas and petroleum coke. Only about half of the energy consumed in an oil refinery is supplied by purchased fuels; the other half is supplied by these in-house by-product fuels. Table B.2 shows the petroleum refining industry's 1978 fuel use by energy source.

TABLE B.1. Industrial Groups in SIC 29 SIC Industry

Industry
Petroleum Refining
Paving Mixtures and Blocks
Asphalt Felts and Coatings
Lubricating 0ils and Greases
Products of Petroleum and Coal,
Not Elsewhere Classified


TABLE B.2. Petroleum Refining Industry Fuel Use - 1978

(General Accounting Office 1980)

\begin{tabular}{lrr}
\multicolumn{1}{c}{ Energy Source } & $10^{12}$ Btu & $\%$ \\
Refinery Gas & 1291.7 & 42.2 \\
Natural Gas & 820.6 & 26.8 \\
Petroleum Coke & 394.8 & 12.9 \\
Residual Fuel 0il & 314.6 & 10.3 \\
Purchased Electricity & 94.8 & 3.1 \\
Liquid Petroleum Gas & 57.1 & 1.9 \\
Distillate Fuel 0il & 51.7 & 1.7 \\
Purchased Steam & 32.9 & 1.0 \\
Coal & 3.2 & 0.1 \\
Crude 0il & 2.6 & 0.0 \\
& 3064.0 & 100.0
\end{tabular}

High-temperature thermal energy requirements are met with fired heaters, and lower-temperature requirements are met with process steam. Although considerable steam is used throughout the refining process, the ratio of direct heat use to steam use is approximately 4 to 1 . Steam pressures range from 200 to $600 \mathrm{psig}$, with the moderate-to-high-pressure steam being used to supply shaft power, while the lower-pressure steam is used for steam stripping, vacuum condensation, small pumps, and heat exchangers. Steam is generated in both direct-fired boilers and waste-heat boilers. A small amount of high-pressure steam in the 700-1400 psig range is also produced for electrical power generation and shaft power (United Technologies Corporation 1980). Highpressure steam is usually used in extraction turbines, with exhausts used for process heat. Only a small amount of hot water is needed for salt stripping of certain crudes. Steam demand is relatively constant throughout the year (except for space heating requirements).

The following sections describe the major processes, heat-transfer operations, and waste heat streams associated with petroleum refining. 


\section{PETROLEUM REFINING}

The petroleum refining industry (SIC 2911), as defined by the U.S. Department of Commerce, includes establishments that are primarily engaged in producing gasoline, kerosene, distillate fuel oils, residual fuel oils, lubricants, and other products from crude petroleum and its fractionation products, through straight distillation of crude $0 i 1$, redistillation of unfinished petroleum derivatives, cracking, or other processes. Establishments that are primarily engaged in producing natural gasoline from natural gas are classified as mining industries. Those that manufacture lubricating oils and greases by blending and compounding purchased materials are included in SIC 2992. Establishments that primarily re-refine used lubricating oils are also included in SIC 2992.

As of January 1, 1982 there were 274 operating petroleum refineries in the United States ("Annual Refining Survey" 1982). Table B.3 shows that these refineries range in size from only 600 barrels per stream-day ( $B / D$ ) to over $500,000 \mathrm{~B} / \mathrm{D}$. The total U.S. refining capacity as of January 1, 1982, was about $18,625,700 \mathrm{~B} / \mathrm{D}$. The states with the largest petroleum refining capacities are Texas, Louisiana, California, Illinois, Pennsylvania, New Jersey, Oklahoma, Ohio, Indiana, and Kansas. Although each refinery is characterized by a unique capacity, processing configuration, and product distribution, there are some generic similarities among refineries in a given geographic region, primarily due to the characteristics of the crude oil processed and the products distributed.

Figure B.1 provides a generalized process flow diagram for a large integrated refinery (Hamel and Brown, 1979). Table B.4 provides a summary of energy requirements by major process for the representative refinery depicted in Figure B.1. Values given are based on industry-wide averages for the specific processes involved. It should be re-emphasized that both the processes depicted and the values shown will vary from refinery to refinery. 
TABLE B.3 Size Distribution of Operating U.S. Petroleum Refineries (as of January 1, 1982)

\begin{tabular}{|c|c|c|c|c|c|c|}
\hline $\begin{array}{c}\text { Refinery } \\
\text { Size } \\
\left(10^{3} \mathrm{~B} / \mathrm{D}\right)^{\mathrm{a}} \\
\end{array}$ & $\begin{array}{l}\text { Number of } \\
\text { Refineries } \\
\end{array}$ & $\begin{array}{l}\% \text { of Total } \\
\text { Refineries } \\
\end{array}$ & $\begin{array}{l}\text { Cumulative } \\
\text { Total (\%) }\end{array}$ & $\begin{array}{l}\text { Total } \\
\text { Capacity } \\
(B / D)\end{array}$ & $\begin{array}{l}\text { \% of Total } \\
\text { Capacity }\end{array}$ & $\begin{array}{l}\text { Cumulative } \\
\text { Total }(\%)\end{array}$ \\
\hline $0-1$ & 1 & 0.4 & 0.4 & 600 & 0.0 & 0.0 \\
\hline $1-2$ & 3 & 1.1 & 1.5 & 3,800 & 0.0 & 0.0 \\
\hline $2-5$ & 16 & 5.8 & 7.3 & 68,900 & 0.4 & 0.4 \\
\hline $5-10$ & 37 & 13.5 & 20.8 & 283,500 & 1.5 & 1.9 \\
\hline $10-20$ & 45 & 16.4 & 37.2 & 660,700 & 3.5 & 5.4 \\
\hline $20-30$ & 32 & 11.7 & 48.9 & 808,500 & 4.3 & 9.7 \\
\hline $30-50$ & 42 & 15.3 & 64.2 & $1,768,000$ & 9.5 & 19.2 \\
\hline $50-75$ & 29 & 10.6 & 74.8 & $1,792,600$ & 9.6 & 28.8 \\
\hline $75-100$ & 13 & 4.7 & 79.5 & $1,127,200$ & 6.1 & 34.9 \\
\hline $100-125$ & 12 & 4.4 & 83.9 & $1,335,700$ & 7.2 & 42.1 \\
\hline $125-175$ & 14 & 5.1 & 89.0 & $1,964,800$ & 10.5 & 52.6 \\
\hline $175-225$ & 12 & 4.4 & 93.4 & $2,284,800$ & 12.3 & 64.9 \\
\hline $225-300$ & 7 & 2.6 & 96.0 & $1,929,700$ & 10.4 & 75.3 \\
\hline $300-400$ & 5 & 1.8 & 97.8 & $1,720,600$ & 9.2 & 84.5 \\
\hline $400-500$ & 4 & 1.5 & 99.3 & $1,688,300$ & 9.1 & 93.6 \\
\hline $500+\mathrm{TC}$ & $\frac{2}{274}$ & $\frac{0.7}{100.0}$ & 100.0 & $\frac{1,188,000}{18,625,700}$ & $\frac{6.4}{100.0}$ & 100.0 \\
\hline
\end{tabular}

a. $B / D=$ Barrels per stream-day. This represents the amount of crude oil that a refinery can process when running at full capacity.

Source: "Annual Refining Survey", 0i1 \& Gas Journal, March 22, 1982.

Process Description

In general, petroleum refineries convert crude oil into finished products by employing three fundamental operations: (1) physical separation (distillation, absorption, stripping, and extraction), (2) chemical conversion (changing molecular structure), and (3) treating. Crude oil, as it comes from the ground, is a mixture of thousands of different hydrocarbons. In addition, 


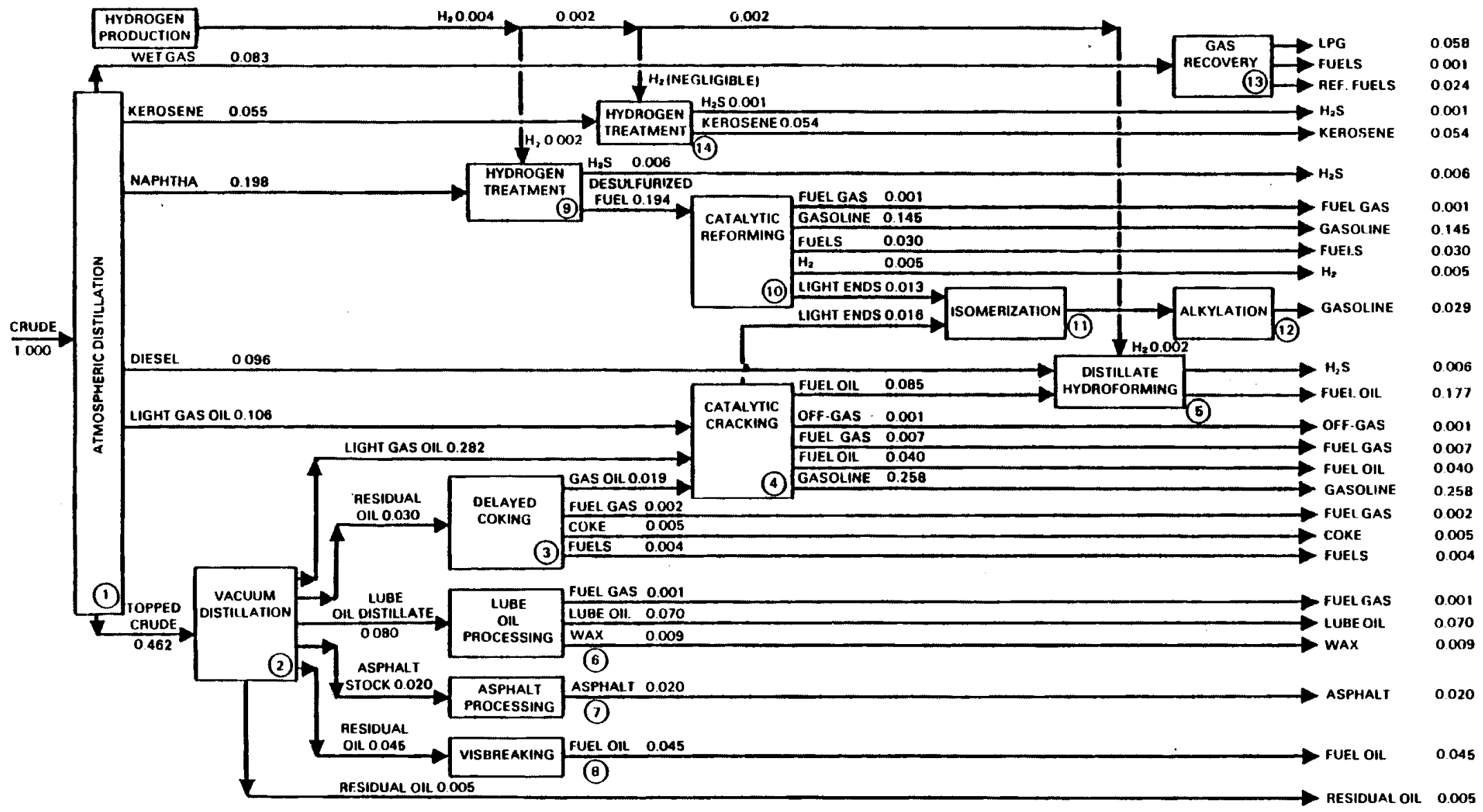

Source: Hamel and Brown, 1979

FIGURE B.1 Process Flow Diagram for Representative Refinery 
TABLE B.4. Total Daily Energy Requirements for Representative Refinery

\begin{tabular}{|c|c|c|}
\hline №. & Process & $\begin{array}{c}\text { Mass } \\
\text { Factor (a) }\end{array}$ \\
\hline 1 & Atmospheric Distillation & 1.000 \\
\hline 2 & Vacuum Distillation & 0.462 \\
\hline 3 & Delayed Coking & 0.030 \\
\hline 4 & Catalytic Cracking & 0.407 \\
\hline 5 & Distillate Hydroforming & 0.181 \\
\hline 6 & Lube 0il Processing & 0.080 \\
\hline 7 & Asphalt Processing & 0.020 \\
\hline 8 & Visbreaking & 0.045 \\
\hline 9 & Naphtha Hydrotreating & 0.198 \\
\hline 10 & Catalytic Reforming & 0.194 \\
\hline 11 & Isomerization & 0.029 \\
\hline 12 & Alkylation & 0.029 \\
\hline 13 & Gas Recovery & 0.083 \\
\hline 14 & Kerosene Hydrotreating & ILS \\
\hline
\end{tabular}

\begin{tabular}{|c|c|c|c|c|c|c|}
\hline \multirow[b]{2}{*}{$\begin{array}{r}\text { Charge } \\
B / 0 \times 10^{3} \\
\end{array}$} & \multicolumn{2}{|c|}{ Steam $^{(b)}$} & \multicolumn{2}{|c|}{ Direct Heat } & \multicolumn{2}{|c|}{ Electricity } \\
\hline & $\begin{array}{l}\text { LB/BBL } \\
\text { Charge }\end{array}$ & $\begin{array}{l}\text { Total LB } \\
\times 10^{6} \\
\end{array}$ & $\begin{array}{l}\text { BTU/BBL } \\
\times 10^{3} \\
\end{array}$ & $\begin{array}{r}\text { Total } \\
\text { BTU } \times 10^{6} \\
\end{array}$ & $\mathrm{KWH} / \mathrm{BBL}$ & $\begin{array}{l}\text { Total } \\
\mathrm{KWH} \times 10^{3} \\
\end{array}$ \\
\hline 250.00 & $25^{(c)}$ & 6.25 & $159^{(c)}$ & 39,750 & $0.94^{(c)}$ & 235.0 \\
\hline 115.50 & $28^{(c)}$ & 3.23 & $103^{(c)}$ & 11,897 & $0.85^{(c)}$ & 98.2 \\
\hline 7.50 & $19^{(c)}$ & 0.14 & $455^{(c)}$ & 3,413 & $1.70^{(c)}$ & 12.8 \\
\hline 101.75 & $116^{(c)}$ & 11.80 & $235^{(c)}$ & 23,911 & $6.05^{(c)}$ & 615.6 \\
\hline 45.25 & $(8)^{(d)}$ & $(0.36)$ & ${ }_{10}^{(d)}$ & 453 & $2.30^{(d)}$ & 104.1 \\
\hline 20.00 & $14^{(d)}$ & 0.28 & $137^{(d)}$ & 2,740 & $8.93^{(d)}$ & 178.6 \\
\hline 5.00 & $50^{(e)}$ & 0.25 & $208^{(e)}$ & 1,040 & $2.55^{(\mathrm{e})}$ & 12.8 \\
\hline 11.25 & $42^{(c)}$ & 0.47 & $244^{(c)}$ & 2,745 & $1.05(c)$ & 11.8 \\
\hline 49.50 & $11^{(f)}$ & 0.54 & $53^{(f)}$ & 2,624 & $1.00^{(f)}$ & 49.5 \\
\hline 48.50 & $61^{(c)}$ & 2.96 & $450^{(c)}$ & 21,825 & $3.15^{(c)}$ & 152.8 \\
\hline 7.25 & $322^{(c)}$ & 2.33 & $270^{(c)}$ & 1,958 & $3.05^{(c)}$ & 22.1 \\
\hline 7.25 & $500^{(c)}$ & 3.63 & $720^{(c)}$ & 5,220 & $5.75^{(c)}$ & 41.7 \\
\hline 20.75 & None ${ }^{(g)}$ & None & $62^{(g)}$ & 1,287 & $0.53^{(g)}$ & 11.0 \\
\hline 13.75 & $25^{(f)}$ & $\frac{0.34}{31.86}$ & $66^{(f)}$ & $\begin{array}{r}908 \\
119,771\end{array}$ & $1.70^{(f)}$ & $\frac{23.4}{1,569.4}$ \\
\hline
\end{tabular}

NOTES: (a) Hamel and Brown, 1979.

(b) Assume 10\% of total steam used for mechanical drives.

(c) United Technologies Corporation, 1980.

(d) "1982 Refining Process Handbook", 1982.

(e) Battelle, Columbus Laboratories and Battelle, Pacific Northwest Laboratories, 1976.

(f) Palmer, et al., 1980.

(g) Haynes, 1976. 
there may be large amounts of paraffin wax, asphaltic matter, sulfur, salts, and other impurities. It is necessary to separate the complex mixture into various cuts of different boiling ranges before any chemical rearrangements are attempted.

\section{Physical Separation}

Fractional distillation is based upon the fact that compounds with greater molecular weights have higher boiling points than compounds with lower molecular weights. The crude oil is partially vaporized in the tubes of a furnace and the two-phase mixture then enters the crude unit (distillation column). As the vapors rise, they cool and condense at various levels of the column depending upon their relative volatilities. Light hydrocarbons $\left(\mathrm{C}_{4}{ }^{-}\right)^{(a)}$ rise to the top of the column where they are liquified in a condenser and recovered in a reflux drum. In between the condenser at the top and the reboiler at the bottom of the column, various sidecuts are drawn off, depending upon the particular product slate of the refinery. The heaviest fractions of the crude oil drop to the bottom of the column and are withdrawn.

Hydrocarbons with roughly fourteen or more carbon atoms cannot be boiled at atmospheric pressure without decomposing. Thus, the heavier crude fractions must be distilled under a vacuum. Because vacuum distillation equipment is more expensive than atmospheric distillation equipment, most crude units consist of two columns. The bottoms from the atmospheric column are pumped to the vacuum column, where the heavy fractions are split into several boiling ranges. The vacuum column bottoms are used in the manufacture of asphalt.

\section{Chemical Conversion}

Once the crude oil has been separated, the various fractions are piped to different plants in the refinery. In general, the next step is some type of chemical conversion process, where the hydrocarbon molecules are rearranged to form desired petroleum products such as gasoline. The importance of chemical conversion was realized at the beginning of the 20 th century with the advent of

(a) $\mathrm{C}_{4}^{-}$refers to all compounds that have boiling points less than or equal to the boiling point of normal butane. 
the automobile. In 1920, the gasoline yield from each barrel ${ }^{(a)}$ of crude was only 11 gallons. Today refineries are able to produce an average of 19 gallons of gasoline per barrel of crude. The major processes which enable refineries to produce gasoline from hydrocarbons that are not in the gasoline boiling range $^{(b)}$ are thermal cracking, catalytic cracking, hydrocracking, reforming, alkylation, polymerization, and isomerization.

Thermal cracking was the first conversion process introduced in $0 i 1$ refineries. In this process, heavy fractions of the crude are subjected to high temperatures and pressures, resulting in the breaking of molecular bonds. Thus, large complex molecules are cracked into smaller molecules. Cracking produces gasoline which is superior in antiknock characteristics to the gasoline obtained by simple fractionation of the crude. Although thermal cracking gives a low rate of conversion and is not used to a great extent anymore, it is quite important from an historical standpoint. After the first successful thermal cracker was put into operation in 1913, the refiners began to realize the extent of their capability to alter the petroleum molecules in a useful way.

The second major conversion process, brought to light in 1937, was catalytic cracking. This process accomplishes the same objective as thermal cracking, but the use of a selective catalyst enables cracking to occur at moderate pressures. Also, the gasoline produced has an even higher octane rating than that made in a thermal cracker. Catalysts used in various catalytic cracking processes range from aluminum and platinum to acids and processed clay.

The most modern and most complex of the cracking processes is called hydrocracking. It is also the most efficient conversion process in terms of extending the usefulness of a barrel of crude 011 . The first hydrocracker was put into service in 1959, and the process was described as "a method of cracking in the presence of hydrogen and a catalyst to convert low-value, high-boiling

(a) A barrel, as used in the petroleum industry, is equal to 42 U.S. gallons. (b) The gasoline boiling range is $100-400^{\circ} \mathrm{F}$. 
distillates to lower-boiling higher-value liquid products with essentially no waste gas produced" (Unzelman and Gerber 1965). In a hydrocracker, hydrocarbons are heated under pressure in the presence of hydrogen and a catalyst. The hydrocracking catalyst has two functions: (1) to crack the molecules, and (2) to hydrogenate the unsaturated products. Most of the products formed are isoparaffins, which make good gasoline.

Secondary reactions also occur in the hydrocracking process. The desulfurization reaction produces $\mathrm{H}_{2} \mathrm{~S}$, and the denitrification reaction produces $\mathrm{NH}_{3}$. Water is injected into the reactor effluent stream to force the $\mathrm{H}_{2} \mathrm{~S}$ and $\mathrm{NH}_{3}$ into an aqueous phase. The stream then goes to a separator which removes the aqueous phase from the organic phase and sends it to a waste water treating plant.

The quality of a motor gasoline blend is a function of its volatility and its knock characteristics. The tendency of a fuel to knock in an engine is classified numerically as the fuel's octane number. The higher a gasoline's octane number, the less the tendency to knock and the better the performance expected in an engine. The reference points for octane rating are iso-octane at 100, and normal-heptane at 0 . The octane number of "straight-run" gasoline from the crude unit and "cracked" gasoline from the cracking units is raised by feeding these stocks to a catalytic reformer.

The catalytic reformer's main functions are to produce aromatics and to reform normal paraffins into isoparaffins, which causes an increase in octane number. The reforming reactions occur at elevated temperatures and pressures in the presence of hydrogen and a platinum catalyst. Reforming results in molecular rearrangement; the total number of carbon atoms in the rearranged molecule is not altered, and large quantities of hydrogen are produced as a by-product.

Since large volumes of light hydrocarbon gases are produced in a typical refinery, it is common to see alkylation, polymerization, and/or isomerization units present. The first two of these processes are methods of combining small molecules to make larger ones. Isomerization is a molecular rearrangement process which produces feedstock for alkylation units or high-octane isoparaffins 
for gasoline blending. Prior to the 1930s, the light gas streams were burned as fuel. Now alkylation, polymerization, and isomerization have increased the gasoline yield from a barrel of crude and eliminated the wasteful burning of useful refinery byproducts.

\section{Treating}

The main purpose of treating processes is to remove the odorous and corrosive sulfur compounds from refinery products. The crude oil may be desulfurized before conversion, or the products may be desulfurized individually, depending upon the economics of the situation. Due to strict EPA regulations limiting emission of sulfur compounds into the atmosphere, many refineries are now installing desulfurizers capable of handling all boiling ranges of crude 0i1. These units are similar to hydrocrackers except that the catalyst performs desulfurization and denitrification without causing cracking.

In addition to sulfur removal, gasoline must also undergo other various blending and treating processes in order to meet strict product specifications. Finished gasoline is a blend of straight-run gasoline, cracked gasoline, reformed gasoline, and polymerized gasoline. To this blend, refiners add a variety of additives to improve the quality of the fuel. Among these additives are anti-knock compounds, antirust compounds, anti-icing compounds, and detergents.

Heat-Transfer Operations

Refinery energy consumption varies between 0.3 and 1.0 million Btu per barrel of crude oil input (Nydick, et al., 1976). This variability is largely due to the type of crude oil being refined and the specific process units that are employed.

Most of the heat-transfer operations that take place in petroleum refineries involve heat exchangers, fired heaters, boilers, or condensers. Heat exchangers that transfer heat from a hot product stream to a cold feed stream are assumed to be completely efficient. That is, the heat lost by the hot stream is equal to the heat gained by the cold stream. This type of heattransfer operation will not be discussed further since there is no waste heat 
to be recovered. The following sections describe the heat-transfer operations that occur in fired heaters, boilers, and condensers.

Fired Heaters

As mentioned earlier, most of the thermal energy requirements of a petroleum refinery are met with fired heaters. Basically, fired heaters consist of combustion chambers surrounded by tubes through which the process fluids flow to absorb heat. Tubes in the radiant section receive heat directly from the flame, while tubes in the convection section receive heat from the hot combustion gases. Nelson (1969) has identified eleven basic types of fired heaters that cover a range of capacities and temperature requirements.

Before 1970 fired heaters operated at a net efficiency of about 75 percent. Modern fired heaters with duties of over $20 \times 10^{6} \mathrm{Btu} / \mathrm{hr}$ can achieve a net efficiency of 90-92 percent (Sittig 1977).

Table B.5 shows the direct heat demands of the processes depicted in the representative refinery (Figure B.1). It also shows the ranges of process temperatures reported in the literature for these processes.

Table B.6 shows 1979 energy consumption for direct-heat applications in the petoleum refining industry. Refinery gas and natural gas are the preferred fuels because they burn much cleaner than fuel oil. However, many fired heaters in petroleum refineries have dual-fuel capability and can easily switch from refinery gas to fuel oil as fuel prices change.

Refinery gas is composed of methane, ethane, propane, butane, ethylene, propylene, and butylene. These low-boiling refinery products serve as petrochemical feedstocks as well as refinery fuels.

Refinery fuel oil is the residue from crude oil distillation. It is composed of high-molecular-weight compounds and has more sulfur per pound than other fuels. Typical residual fuel oils include No. 5 and No. 6 heating oils. The ash content of residual fuel oil rarely exceeds 0.2 weight percent (Babcock and Wilcox 1975). 
TABLE B.5 Direct Heat Demand for Representative Refinery

\begin{tabular}{|c|c|c|c|c|c|}
\hline No. & $\begin{array}{l}\text { User } \\
\text { Process Description }\end{array}$ & Medium & $\begin{array}{l}\text { Heat Flow } \\
\left(10^{6} \text { Btu/Hr }\right)\end{array}$ & $\begin{array}{c}\text { Process } \\
\text { Temperature } \\
\left({ }^{\circ} \mathrm{F}\right) \\
\end{array}$ & $\begin{array}{c}\text { Outlet } \\
\text { Conditions } \\
\end{array}$ \\
\hline 1 & Atmospheric Distillation & Fuel & 1656.3 & $600-700^{(a)}$ & Stack Gas \\
\hline 2 & Vacuum Distillation & Fuel & 495.7 & $725-775^{(a)}$ & Stack Gas \\
\hline 3 & Delayed Coking & Fue 1 & 142.2 & $890-960^{(a)}$ & Stack Gas \\
\hline 4 & Catalytic Cracking & Fue 1 & 996.3 & $860-950^{(a)}$ & Stack Gas \\
\hline 5 & Distillate Hydroforming & Fue 1 & 18.9 & $300-700^{(b)}$ & Stack Gas \\
\hline 6 & Lube 0 il Processing & Fue 1 & 114.2 & $390-790^{(b)}$ & Stack Gas \\
\hline 7 & Asphalt Processing & Fue? & 43.3 & $500^{(d)}$ & Stack Gas \\
\hline 8 & Visbreaking & Fue 1 & 114.4 & $860-890^{(a)}$ & Stack Gas \\
\hline 9 & Naphtha Hydrotreating & Fue 1 & 109.3 & $500-800^{(c)}$ & Stack Gas \\
\hline 10 & Catalytic Reforming & Fuel & 909.4 & $850-970^{(a)}$ & Stack Gas \\
\hline 11 & Isomerization & Fue 1 & 81.6 & $100-210^{(a)}$ & Stack Gas \\
\hline 12 & Alkylation & Fue 1 & 217.5 & $70-115^{(a)}$ & Stack Gas \\
\hline 13 & Gas Recovery & Fue 1 & 53.6 & $250-350^{(e)}$ & Stack Gas \\
\hline 14 & Kerosene Hydrotreating & Fue 1 & $\frac{37.8}{4,990.5}$ & $500-800(c)$ & Stack Gas \\
\hline
\end{tabular}

(a) United Technologies Corporation, 1980.

(b) "1.982 Refining Process Handbook", 1982.

(c) Palmer, et al., 1980.

(d) Battelie, Columbus Laboratories and Battelle, Pacific Northwest Laboratories, 1976.

(e) Haynes, 1976.

\section{Boilers}

Steam is generated in a petroleum refinery in fired boilers and waste-heat boilers. About 75 percent of a refinery's total steam requirements is generated in fired boilers, and 25 percent is generated in waste-heat boilers (Nydick et al. 1976). 
TABLE B.6. 1979 Energy Consumption in Direct-Heat Applications in the Petroleum Industry (Energy and Environmental Analysis, Inc., 1983)

Fuel Type

Refinery Gas

Natural Gas

Distillate Fuel $0 i 1$

Residual Fuel $0 i 1$

Liquefied Petroleum Gas

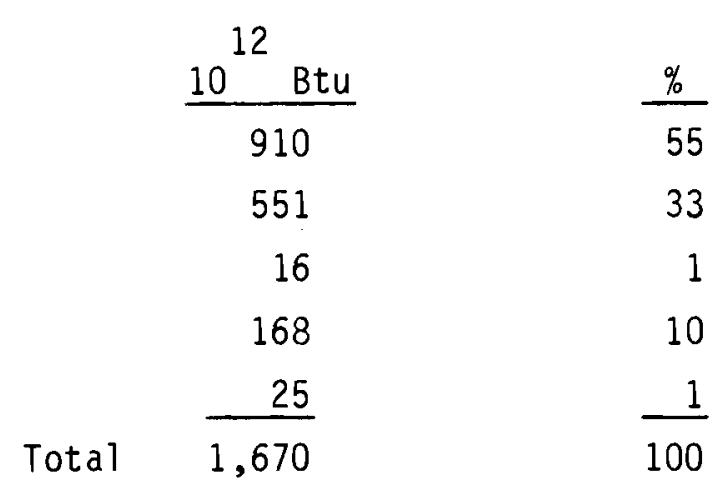

Package boilers are available in sizes up to $80,000 \mathrm{lb} / \mathrm{hr}$ for pressures up to 600 psig. Built-in-place boilers are available in a full range of sizes and pressure ratings (Bland and Davidson 1967). Table B.7 shows the steam demands of the processes depicted in Figure B.1.

\section{Fired Boilers}

As shown earlier in Table B.2, refinery gas and natural gas represented almost 70 percent of the fuels used by the petroleum refining industry in 1978. Petroleum coke represented about 13 percent of the fuel usage, and residual fuel oil represented about 10 percent. Many refinery boilers have dual-fuel capability and can easily switch from refinery gas to fuel oil as fuel prices change. Refinery gas and natural gas are the preferred boiler fuels because they burn much cleaner than fuel oil or coke.

Petroleum coke is the product of delayed coking. The ash content of the coke can vary from 0.1 to 2.8 weight percent, and the sulfur content can vary from 0.5 to 7.0 weight percent, depending upon the specific coking process employed (Gaines et al. 1981).

Almost no salable petroleum coke is burned in petroleum refineries. The fuels used to generate steam include distillate fuel oil, residual fuel oil, natural gas, liquefied petroleum gas (LPG), and refinery gas. The natural gas, 
TABLE B.7. Steam Demand for Representative Refinery

\begin{tabular}{|c|c|c|c|c|c|c|c|c|}
\hline No. & $\begin{array}{l}\text { User } \\
\text { Process Description }\end{array}$ & Medium & $\begin{array}{l}\text { Pressure } \\
\text { (psig) }\end{array}$ & $\begin{array}{l}\text { Temp. } \\
\left({ }^{\circ} \mathrm{F}\right)\end{array}$ & $\begin{array}{c}\text { Heat } \\
\text { Content } \\
\text { (Btu/lb) }\end{array}$ & $\begin{array}{l}\text { Mass Flow } \\
\left(10^{3} \mathrm{lb} / \mathrm{hr}\right)\end{array}$ & $\begin{array}{l}\text { Heat Flow } \\
\left(10^{6} \mathrm{Btu} / \mathrm{hr}\right)\end{array}$ & Outlet Conditions \\
\hline 1 & Atmospheric Distillation & Steam & 385 & 500 & 1245 & 234.4 & 259.5 & $\begin{array}{l}45 \% \text { Cond. } 170^{\circ} \mathrm{F} \\
(138 \mathrm{Btu} / \mathrm{lb})\end{array}$ \\
\hline 2 & Vacuum Distillation & Steam & 385 & 500 & 1245 & 121.1 & 134.1 & $\begin{array}{l}45 \% \text { Cond. } 170^{\circ} \mathrm{F} \\
(138 \mathrm{Btu} / \mathrm{b})\end{array}$ \\
\hline 3 & Delayed Coking & Steam & 385 & 500 & 1245 & 5.3 & 5.9 & $\begin{array}{l}45 \% \text { Cond. } \stackrel{\circ}{1} 170^{\circ} \mathrm{F} \\
(138 \text { Btu/lb) }\end{array}$ \\
\hline 4 & Catalytic Cracking & Steam & 385 & 500 & 1245 & 442.5 & 489.8 & $\begin{array}{l}45 \% \text { Cond. } 170^{\circ} \mathrm{F} \\
(138 \mathrm{Btu} / 1 \mathrm{~b})\end{array}$ \\
\hline 5 & Distillate Hydroforming & Steam & 385 & 500 & 1245 & $(13.5)$ & $(14.9)$ & $\begin{array}{l}45 \% \text { Cond. } 170^{\circ} \mathrm{F} \\
(138 \mathrm{Btu} / 1 \mathrm{~b})\end{array}$ \\
\hline 6 & Lube 0il. Processing & Steam & 385 & 500 & 1245 & 10.5 & 11.6 & $\begin{array}{l}45 \% \text { Cond. } 170^{\circ} \mathrm{F} \\
(138 \mathrm{Btu} / 1 \mathrm{~b})\end{array}$ \\
\hline 7 & Asphalt Processing & Steam & 385 & 500 & 1245 & 9.4 & 10.4 & $\begin{array}{l}45 \% \text { Cond. } 170^{\circ} \mathrm{F} \\
(138 \mathrm{Btu} / 1 \mathrm{~b})\end{array}$ \\
\hline 8 & Visbreaking & Steam & 385 & 500 & 1245 & 17.6 & 19.5 & $\begin{array}{l}45 \% \text { Cond. } 170^{\circ} \mathrm{F} \\
(138 \mathrm{Btu} / 1 \mathrm{~b})\end{array}$ \\
\hline 9 & Naphtha Hydrotreating & Steam & 385 & 500 & 1245 & 20.3 & 22.5 & $\begin{array}{l}45 \% \text { Cond. } 170^{\circ} \mathrm{F} \\
(138 \mathrm{Btu} / 1 \mathrm{~b})\end{array}$ \\
\hline 10 & Catalytic Reforming & Steam & 385 & 500 & 1245 & 111.0 & 122.9 & $\begin{array}{l}45 \% \text { Cond. } 170^{\circ} \mathrm{F} \\
(138 \mathrm{Btu} / 1 \mathrm{~b})\end{array}$ \\
\hline 11 & Isomerization & Steam & 385 & 500 & 1245 & 87.4 & 96.8 & $\begin{array}{l}45 \% \text { Cond. } 170^{\circ} \mathrm{F} \\
(138 \text { Btu/lb) }\end{array}$ \\
\hline 12 & Alkylation & Steam & 385 & 500 & 1245 & 136.1 & 150.7 & $\begin{array}{l}45 \% \text { Cond. } 170^{\circ} \mathrm{F} \\
(138 \mathrm{Btu} / \mathrm{lb})\end{array}$ \\
\hline 14 & $\begin{array}{l}\text { Kerosene Hydrotreating } \\
\text { Mechanical (Total for All }\end{array}$ & $\begin{array}{c}\text { Steam } \\
\text { Processes }\end{array}$ & $.5^{385}$ & 500 & 1245 & $\frac{12.8}{1.194 .9}$ & $\frac{14.2}{1.323 .0}$ & $\begin{array}{l}45 \% \text { Cond. a } 170^{\circ} \mathrm{F} \\
(138 \mathrm{Btu} / \mathrm{lb})\end{array}$ \\
\hline & Shaft Power & Steam & 705 & 760 & 1379 & 132.9 & 164.9 & $\begin{array}{l}\text { Cond. } 170^{\circ} \mathrm{F} \\
(138 \mathrm{Btu} / 1 \mathrm{~b})\end{array}$ \\
\hline & Elec. Gen. $660 \%$ Turb. Eff & Steam & ALS $^{705}$ & 760 & 1379 & $\frac{229.7}{432.6}$ & $\frac{371.9}{536.8}$ & $\begin{array}{l}\text { Cond. } 170^{\circ} \mathrm{F} \\
(138 \mathrm{Btu} / 1 \mathrm{~b})\end{array}$ \\
\hline
\end{tabular}


$L P G$, and refinery gas are sometimes fired as a mixture. 0il is probably used more often as a boiler fuel than as a fuel for fired heaters (Nydick et al. 1976).

Even though the ash content of residual fuel oil rarely exceeds 0.2 weight percent, it can still cause significant fireside fouling and corrosion problems in boilers. Compounds of vanadium, sodium, and sulfur have been found in almost every deposit in boilers fired by residual fuel oil. The fouling rates and the thickness of the deposits have been correlated with the sodium-vanadium ratio in the fuel oil, as well as with the velocity and temperature of the flue gases and the tubewall temperatures (Babcock and Wilcox 1975).

Waste-Heat Boilers

Waste-heat boilers in petroleum refineries recover waste heat from process streams or from stack gases leaving a fired heater or catalyst regenerator. Frequently, CO (carbon monoxide) boilers are installed in the regeneration section of fluid catalytic cracking units (FCC). When coke is burned from the FCC catalyst in the regeneration operation, the catalyst reaches a temperature of $1100-1200^{\circ} \mathrm{F}$. The hot of fgas from this combustion contains about 8-10 percent carbon monoxide. This gas is burned with about 10 percent supplemental fuel in a waste-heat boiler (Nydick et al. 1976). Catalyst fines suspended in the flue gas from a fluid catalytic cracker foul the tube walls of the co boiler, hindering heat transfer and necessitating shutdown every five months for cleaning (Farrar Undated).

Waste-heat boilers are sometimes employed to generate steam from the following sources of waste heat: fluid coking plants, sulfur recovery plants, hydrogen manufacturing plants (steam-methane or steam-naphtha reformers), the exhaust from gas turbines, recirculating sidecuts from distillation columns, and high-temperature product streams (Nydick et al. 1976).

\section{Condensers}

A petroleum refinery uses large volumes of cooling water. It has been estimated that 80 to $85 \%$ of the total water required by a refinery is for cooling if cooling towers are used for water conservation. Most refineries use 
open circulating cooling systems. Once-through cooling is rare; it is only used at a few coastal refineries that were designed to use seawater (Kermmer 1979).

The separation and conversion processes described earlier require large amounts of heat. In the interest of energy conservation, the hot product streams are often used to preheat the cold feed streams in feed-effluent exchangers. If additional cooling of the hot product streams is required for either process-related or safety-related reasons, it is provided by cooling water.

Cooling water is used extensively to condense the overhead vapors from distillation columns. Barometric condensers are used on some vacuum distillation columns, resulting in intimate mixing of hydrocarbons, steam, and cooling water. Newer refineries use surface condensers (shell-and-tube exchangers) in which the cooling water and the condensate are not in direct contact (Kemmer 1979).

Most condensers and coolers used in petroleum refineries are shell-andtube exchangers. The cooling water is normally on the tube side and the vapor stream is on the shell side. Thus, any scale or other deposits will occur in the tubes, where cleaning is easier. Also, the vapor will pass through the shel1 without excessive pressure drop (Bland and Davidson 1967).

Water-side deposits in overhead and side-product condensers and coolers may contain dead algae from the cooling tower, iron oxide, oil from contamination, carbonates, silica or silicates, and phosphates from cooling-watertreatment chemicals. Just about any combination of these can be expected on the water side (Bland and Davidson 1967).

Waste Heat Streams

According to a 1976 Thermo Electron study (Nydick et al. 1976), there are only two significant potential sources of waste heat in a petroleum refinery: (1) hot flue gases from fired heaters, and (2) hot liquid product streams that are currently cooled to safe storage temperatures by water or air. Other studies have come to the same conclusion, as discussed below. 
Types and Quantities of Waste Heat

In 1979 Gulf Research and Development Company published a User's Guide that contains a step-by-step procedure for preparing an energy profile at any petroleum refinery (Maier et al. 1979). It explains how to perform a detailed refinery energy balance showing all inputs and losses for the total refinery and for the individual processes. Gulf conducted actual studies on the operating equipment in its Alliance refinery. Table B. 8 shows the Alliance refinery energy profile. At the time that the data were taken, about 74 percent of the energy losses at this refinery were from heater and boiler stacks and from air

TABLE B.8. Alliance Refinery Energy Profile (Maier et al. 1979)

Energy Input to Plant

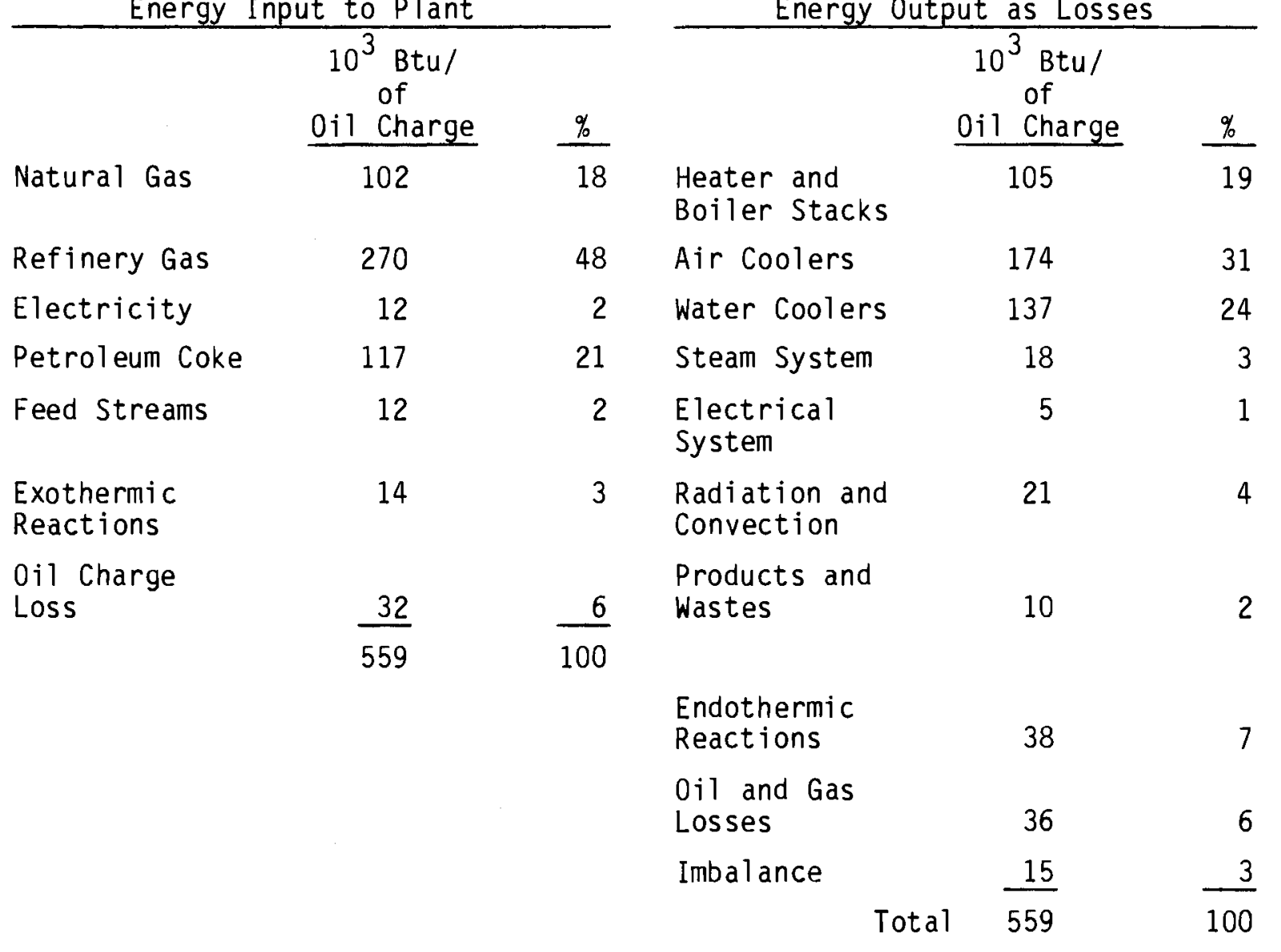


and water coolers. Of course, only part of this energy could be recovered with existing technology. A large part of it is low-level heat that would require the development of advanced waste-heat-recovery techniques.

Latour and Menningman (1981) assessed the potential for waste heat recovery in the petroleum refining industry for the U.S. Environmental Protection Agency. They found that the major sources of waste heat in petroleum refineries are as follows:

(1) cooling and condensing waters used for heater wall cooling and process stream condensing

(2) flue gases from fired heaters

(3) air cooling of recycle and product streams

(4) process wastewaters.

Through a combination of industry correspondence and literature review, they obtained quantitative and qualitative data on these waste streams. Their data show that petroleum refineries discharge about 66.3 percent of their total energy consumption as waste heat. About 53 percent of this waste heat is in non-contact cooling water, 37 percent is in flue gases, and 10 percent is discharged by air cooling of process streams.

Using Latour and Menningman's figures, we can conclude that of its 3 quads/year total energy consumption, the petroleum refining industry rejects about 2 quads of waste heat per year. About 1.1 quad per year of this waste heat is in non-contact cooling water, about 0.7 quad is in flue gases, and about 0.2 quad is discharged by air cooling of process streams.

Another reference (Sittig 1977) states that up to 80 percent of the energy consumption of a petroleum refinery is for fired heater and boiler fuels. Stack gases leave the furnaces at temperatures ranging from $400^{\circ} \mathrm{F}$ to above $1000^{\circ} \mathrm{F}$, representing the major energy losses from the heaters and boilers. Radiation and convection from the fireboxes account for losses of only about 3 percent. 


\section{Temperature Ranges of Waste Heat}

Table B.9 shows the temperature ranges reported in the literature for various petroleum refining waste heat streams. The flue gas streams contain high-temperature waste heat that is probably worth recovering. However, the cooling water, air, and process wastewater streams contain low-temperature waste heat that is probably not worth recovering. The following section presents data on the compositions of various refinery flue gas streams.

Flue Gas Compositions

In 1955 a study was undertaken to determine the quantity and composition of atmospheric emissions from oil refineries in Los Angeles County (U.S. Department of Health, Education, and Welfare 1960). At that time there were 18 refineries in the county with a total combined capacity of over 700,000 barrels of crude oil per day. Table B.10 shows the emissions from 14 fired heaters

TABLE B.9. Petroleum Refining Waste Heat Streams

\begin{tabular}{lc}
\multicolumn{1}{c}{ Source } & Temperature \\
\hline Flue Gases (a) & $700-900^{\circ} \mathrm{F}$ \\
Crude Distillation & $700-950^{\circ} \mathrm{F}$ \\
Catalytic Reforming & $800-1000^{\circ} \mathrm{F}$ \\
Coking & $700-900^{\circ} \mathrm{F}$ \\
Hydrocracking & $600-1000^{\circ} \mathrm{F}$ \\
Hydrogen Production & $300-1000^{\circ} \mathrm{F}$ \\
Others & $150-250^{\circ} \mathrm{F}$ \\
Cooling Water (b) & \\
Air Cooling of Process & \\
Streams to Storage & $250-350^{\circ} \mathrm{F}$ \\
Process Wastewater (c) & $95-125^{\circ} \mathrm{F}$
\end{tabular}

(a) Nydick et al. 1976.

(b) Haynes 1976.

(c) Latour and Menningman 1981. 
TABLE B.10. Emissions from Boilers and Process Heaters in Los Angeles County Refineries (U.S. Department of Health, Education and Welfare 1960)

Oxides of Nitrogen (As $\mathrm{NO}_{2}$ )

Fuel ${ }_{3}$ Gas $\left(\mathrm{Lb} / 10^{3} \mathrm{Ft}^{3}\right)$

Fuel 0 il

Aldehydes (as $\mathrm{HCHO}$ )

0.23

$\underline{(\mathrm{Lb} / \mathrm{Gal})}$

Hydrocarbons (as Hexane)

0.0031

0.068

Organic Acids (as Acetic Acid)

0.026

0.0006

Particulate Matter

0.014

0.0034

0.021

0.011

0.020

Sulfur Dioxide

72 tons/day total for LA County

Sulfur Trioxide

2 tons/day total for LA County

and 10 boilers in the Los Angeles County refineries. Table B.11 summaries the emissions from catalytic cracking unit regenerator stacks in Los Angeles County refineries.

TABLE B.11. Emissions from Catalytic Cracking Unit Regenerator Stacks in Los Angeles County Refineries (U.S. Department of Health, Education, and Welfare 1960)

Hydrocarbons

$$
87-1655 \text { PPM }
$$

Sulfur Dioxide

$65-2190$ PPM

Ammonia

$29-675$ PPM

Aldehydes

$3-177$ PPM

NOX

7 - 394 PPM

Cyanides

$0.07-0.94$ PPM

Carbon Monoxide

$0-7.8 \mathrm{Vol}$. \%

Sulfur Trioxide

2.7 tons/day total for LA County

Total Particulate Matter

5.4 tons/day total for LA County 
In 1976 Arthur D. Little, Inc. did a study of the environmental impacts of potential new process technology in energy-intensive industries for the EPA's Industrial Environmental Research Lab (Arthur D. Little, Inc. 1976). To simulate existing U.S. petroleum refineries, this study developed three cluster models to represent regional characteristics and processing configurations for the East Coast, Gulf Coast, and West Coast. Quantitative data on the sources and composition of airborne emissions from these three model refineries are presented in Tables B.12, B.13, and B.14. 
TABLE B.12. Major Airborne Emissions from East Coast Model Refinery

\begin{tabular}{|c|c|c|c|c|c|c|}
\hline Process/Pollutants & $\begin{array}{l}\text { Particulates } \\
\text { (1b/day) }\end{array}$ & $\begin{array}{c}S 0_{x} \\
(1 b / \text { day })\end{array}$ & $\begin{array}{l}\text { Hydrocarbons } \\
(1 \mathrm{~b} / \text { day })\end{array}$ & $\begin{array}{c}{ }^{N 0} x \\
(1 \mathrm{~b} / \text { day })\end{array}$ & $\begin{array}{l}\text { Aldehydes } \\
\text { (1b/day) }\end{array}$ & $\begin{array}{l}\text { Ammonia } \\
(1 \mathrm{~b} / \text { day })\end{array}$ \\
\hline $\begin{array}{l}\text { - Combustion (heaters and } \\
\text { boilers) }\end{array}$ & 8,979 & 45,200 & 2,671 & 38,085 & 373 & Negligible \\
\hline $\begin{array}{l}\text { - Gas-fired } \\
-\quad 0 i l-f i r e d\end{array}$ & $\begin{array}{r}881 \\
8,098\end{array}$ & --- & $\begin{array}{l}1,321 \\
1,350\end{array}$ & $\begin{array}{l}10,129 \\
27,956\end{array}$ & $\begin{array}{l}132 \\
241\end{array}$ & $\begin{array}{l}\text { Negligible } \\
\text { Negligible }\end{array}$ \\
\hline - Fluid catalytic cracking & 2,780 & 52,800 & 13,684 & 4,416 & 1,182 & 3,359 \\
\hline - Vacuum distillation & Negligible & Negligible & 8,931 & Negligible & Negligible & Negligible \\
\hline Total emissions & $\frac{----}{11.759}$ & $\frac{2,280}{100,280}$ & $\frac{----}{25,286}$ & $\frac{----}{42,501}$ & $\frac{---}{1,555}$ & $\frac{-\cdots-}{3,359}$ \\
\hline
\end{tabular}

Source: Arthur D. Little, Inc., 1976.

$\infty$

TABLE B.13. Major Airborne Emissions from Gulf Coast Model Refinery

\begin{tabular}{|c|c|c|c|c|c|c|}
\hline Process/Pollutants & $\begin{array}{l}\text { Particulates } \\
\text { (lb/day) }\end{array}$ & $\begin{array}{c}S 0_{x} \\
(1 \mathrm{l} / \text { day })\end{array}$ & $\begin{array}{l}\text { Hydrocarbons } \\
\text { (1b/day) }\end{array}$ & $\begin{array}{c}{ }^{N 0} x \\
\text { (1b/day) }\end{array}$ & $\begin{array}{l}\text { Aldehydes } \\
\text { (1b/day) }\end{array}$ & $\begin{array}{l}\text { Ammonia } \\
\text { (ib/day) }\end{array}$ \\
\hline $\begin{array}{l}\text { - Combustion (heaters and } \\
\text { boilers) }\end{array}$ & 9,855 & 27,600 & 2,798 & 40,999 & 398 & Negligible \\
\hline $\begin{array}{l}\text { - Gas-fired } \\
\text { - 0il-fired }\end{array}$ & $\begin{array}{r}867 \\
8,988\end{array}$ & $-\cdots$ & & $\begin{array}{r}9,969 \\
31,030\end{array}$ & $\begin{array}{l}130 \\
268\end{array}$ & \\
\hline - Fluid catalytic cracking & 3,585 & 8,400 & 17,644 & 5,694 & 1,524 & 4,331 \\
\hline - Vacuum distillation & Negligible & Negligible & 9,854 & Negligible & Negligible & Negligible \\
\hline $\begin{array}{l}\text { - Claus plant Total Emissions } \\
\text { To }\end{array}$ & $\frac{---\cdot}{13,440}$ & $\frac{680}{36,680}$ & $\frac{----}{30,296}$ & $\frac{-\cdots}{46,693}$ & $\frac{----}{1,922}$ & $\frac{----}{4,331}$ \\
\hline
\end{tabular}

Source: Arthur D. Little, Inc., 1976. 
TABLE B.14. Major Airborne Emissions from West Coast Model Refinery

\begin{tabular}{|c|c|c|c|c|c|}
\hline $\begin{array}{c}\text { Particulates } \\
\text { (1b/day) }\end{array}$ & $\begin{array}{c}S 0_{x} \\
(1 \mathrm{~b} / \text { day })\end{array}$ & $\begin{array}{l}\text { Hydrocarbons } \\
\text { (1b/day) }\end{array}$ & $\begin{array}{c}{ }^{N 0} \times \\
(1 \mathrm{~b} / \text { day })\end{array}$ & $\begin{array}{l}\text { Aldehydes } \\
\text { (1b/day) }\end{array}$ & $\begin{array}{l}\text { Ammonia } \\
\text { (1b/day) }\end{array}$ \\
\hline 10,765 & 58,800 & 3,055 & 44,769 & 434 & Negligible \\
\hline $\begin{array}{r}945 \\
9,820\end{array}$ & $-\cdots$ & $\begin{array}{l}1,418 \\
1,637\end{array}$ & $\begin{array}{l}10,868 \\
33,901\end{array}$ & $\begin{array}{l}142 \\
292\end{array}$ & $\begin{array}{l}\text { Negligible } \\
\text { Negligible }\end{array}$ \\
\hline 1,708 & 7,600 & 8,404 & 2,712 & 726 & 2,063 \\
\hline Negligible & Negligible & 10,309 & Negligible & Negligible & Negligible \\
\hline$\frac{----}{12,473}$ & $\frac{3,680}{70,080}$ & $\frac{\cdots}{21,763}$ & $\frac{----}{47,481}$ & $\frac{\cdots}{1,160}$ & $\frac{-\cdots}{2,063}$ \\
\hline
\end{tabular}

Source: Arthur D. Little, Inc., 1976. 


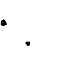

. . 
APPENDIX C

PRIMARY METAL INDUSTRIES - SIC 33 
.

.

, 


\section{APPENDIX C}

PRIMARY METAL INDUSTRIES - SIC 33

The production of primary metal products entails some of the most energy intensive unit operations in the manufacturing sector. SIC 33 accounts for about $20 \%$ of the energy consumed in the manufacturing sector and ranks second in energy consumption at the two-digit SIC level. The principal industries within this classification, and the ones that will be discussed in more detail below, are blast furnaces and steel mills (SIC 3312), gray iron foundries (SIC 3321), primary copper (SIC 3331), and primary aluminum (SIC 3334). These industries are ranked according to their purchases of fuels and electricity in Table C.1. The primary metal industries are characterized by a large number of high-temperature, directly heated unit operations. Each industry typically has several unit operations operating in excess of $1000^{\circ} \mathrm{F} .0$ perating temperatures in the range of $2500^{\circ} \mathrm{F}$ are quite common. Waste energy streams are typically either high-temperature stack gases or low-temperature cooling or process waters. Various types of furnaces are the most common sources of both highand low-temperature waste energy streams. Combustion product stack gases exit the furnace after heating a charge. Cooling water is usually employed to cool furnace walls and other equipment subject to extremely high temperatures.

A more specific description of the manufacturing processes, the major heat transfer operations, and the waste energy streams is provided in the following subsections.

TABLE C.1 Ranking of Primary Metal Industries by Energy Purchases (Bureau of Census 1980)

\begin{tabular}{|c|c|c|c|}
\hline SIC & Name & $\begin{array}{c}\begin{array}{c}\text { Energy } \\
\left(12^{\text {Purchased }}\right. \\
\left(10^{2} \mathrm{Btu}\right)\end{array} \\
\end{array}$ & $\begin{array}{c}\text { Percent of } \\
\text { SIC } 33 \text { Energy } \\
\text { Purchases }\end{array}$ \\
\hline 3312 & $\begin{array}{l}\text { Blast furnaces \& } \\
\text { steel mills }\end{array}$ & 1282 & 56.3 \\
\hline 3334 & Primary aluminum & 360 & 15.8 \\
\hline 3321 & Gray iron foundries & 111 & 4.9 \\
\hline 3331 & Primary copper & 53 & 2.3 \\
\hline
\end{tabular}


BLAST FURNACES AND STEEL MILLS - SIC 3312

According to the 1977 Census of Manufactures (U.S. Department of Commerce 1981), there were 504 establishments involved in some phase of the production of iron and steel at that time. The annual shipments for 1977 amounted to 91 million tons, half of which were produced by 35 fully integrated steel mills. In recent years, annual production has fluctuated around 100 million tons per year; currently, the annual production rate is about 85 million tons of steel (Wall Street Journal, Sept 7, 1983). The relative magnitude of energy consumption and potential for waste heat recovery in the iron and steel industry cannot be overemphasized. Blast furnaces and steel mills consume over $50 \%$ of the fuels and electricity purchased by the primary metal industries, but this figure does not include fuels produced and consumed at the same establishment, fuels used as raw materials, or fuel converted into other fuel types such as coal converted to coke. Inclusion of these additional energy consumption components more than doubles the total amount of energy consumed in SIC 3312 to 2.7 quads (Bureau of Census 1980).

Process Description

An integrated steel mill is a rather complex combination of unit operations that transform iron ore and other raw materials into finished steel products. The major steel mill operations are materials preparation (sintering and pelletizing), coking, blast (reduction) furnace, steel making furnaces (open hearth, electric, basic oxygen), casting (continuous or ingot casting coupled with a roughing mill), rolling and finishing. These operational steps are shown in Figure C.1.

Sintering and pelletizing serves to improve iron ore permeability and gassolid content in the blast furnace. Iron ore fines, limestone fines and coke are sintered on a traveling grate to produce lumps about the size of golf balls. Similarly sized pellets are produced in a balling drum from iron ore and bentonite. The pellets are then coated with pulverized coal and ignited on a traveling grate furnace. Coke is prepared for the blast furnace by the destructive distillation of coal in the coke ovens. The coal is heated externally by coke oven gas produced from previous batches. Iron ore, sinter 


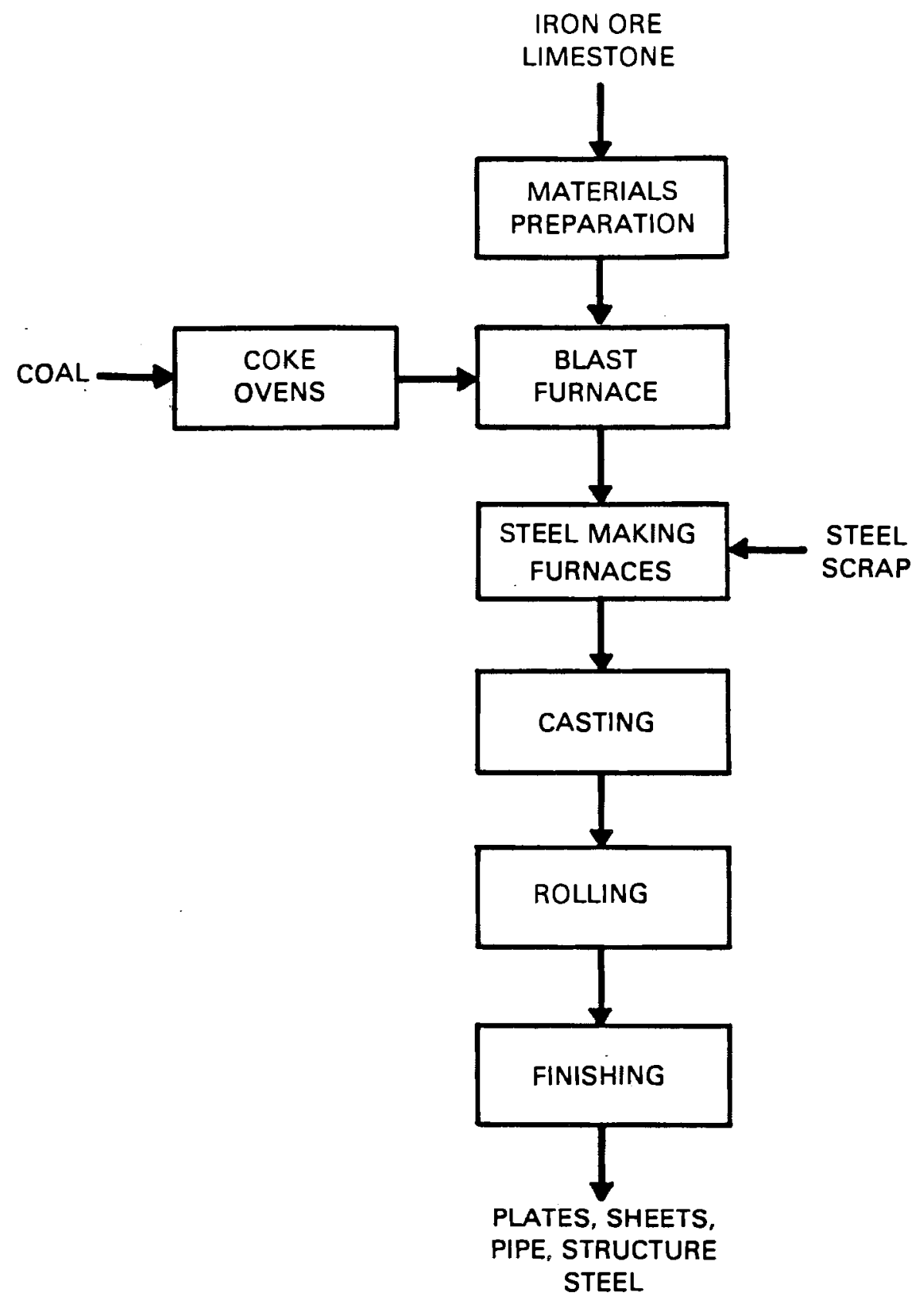

FIGURE C.1 Major Steel Mill Operations

pellets, coke and fluxing agents are charged to the blast furnace where iron oxides are reduced to pig iron. Coke is both the reducing agent and the major supplier of heat to melt the iron. The object of steel making is to oxidize 
excessive amounts of carbon, silicon, manganese, phosphorus, and other impurities from pig iron and scrap charges. Over the years, the efficiency of the basic oxygen furnace caused the demise of the open hearth furnace as the principal steelmaking operation. Electric furnaces are used principally for recycling steel scrap.

Molten steel from the steelmaking furnaces would be cast into ingots and subsequentiy rolled into blooms, billets, and slabs in a conventionally operated mi11. The energy costs associated with ingot soaking have pushed mill operators toward continuous casting in recent years. In the final steps of an integrated steel mill, blooms, billets, and slabs are distributed to separate rolling and finishing lines to produce plates, sheets, pipes and other structural steel products.

Heat Transfer Operations

An integrated steel mill is one of the largest energy-consuming industrial processes. The significant unit operations from a heat transfer standpoint are coking, agglomeration, blast furnace, open hearth furnace, soaking, reheating, and annealing. Each of these unit operations operate through direct-contact heat exchange, except for coking. Process details are discussed in the following paragraphs.

\section{Coking}

Coke is required in the blast furnace for the reduction of iron bearing material to pig iron. Coke is produced from coal via the by-product or retort method. A coking oven is a long, narrow, refractory lined chamber, typically 30 to 40 feet long, 10 to 20 feet high, and 15 to 20 inches wide. A coking plant will usually have 50 to 60 ovens operating together in what is known as a battery. Pulverized coal is charged to the coking ovens, which are then sealed. Previously generated coke oven gas is burned to externally heat the chamber. The by-products (coke oven gas, tars, oils) are recovered separately for their energy content. After about 18 hours, the coal has fused into coke; the doors of the oven are opened; and a ram pushes the coke into a quenching car. The coke is quenched with water and allowed to cool before being charged to the blast furnace. A drawing of a coke oven is shown in Figure C.2. 


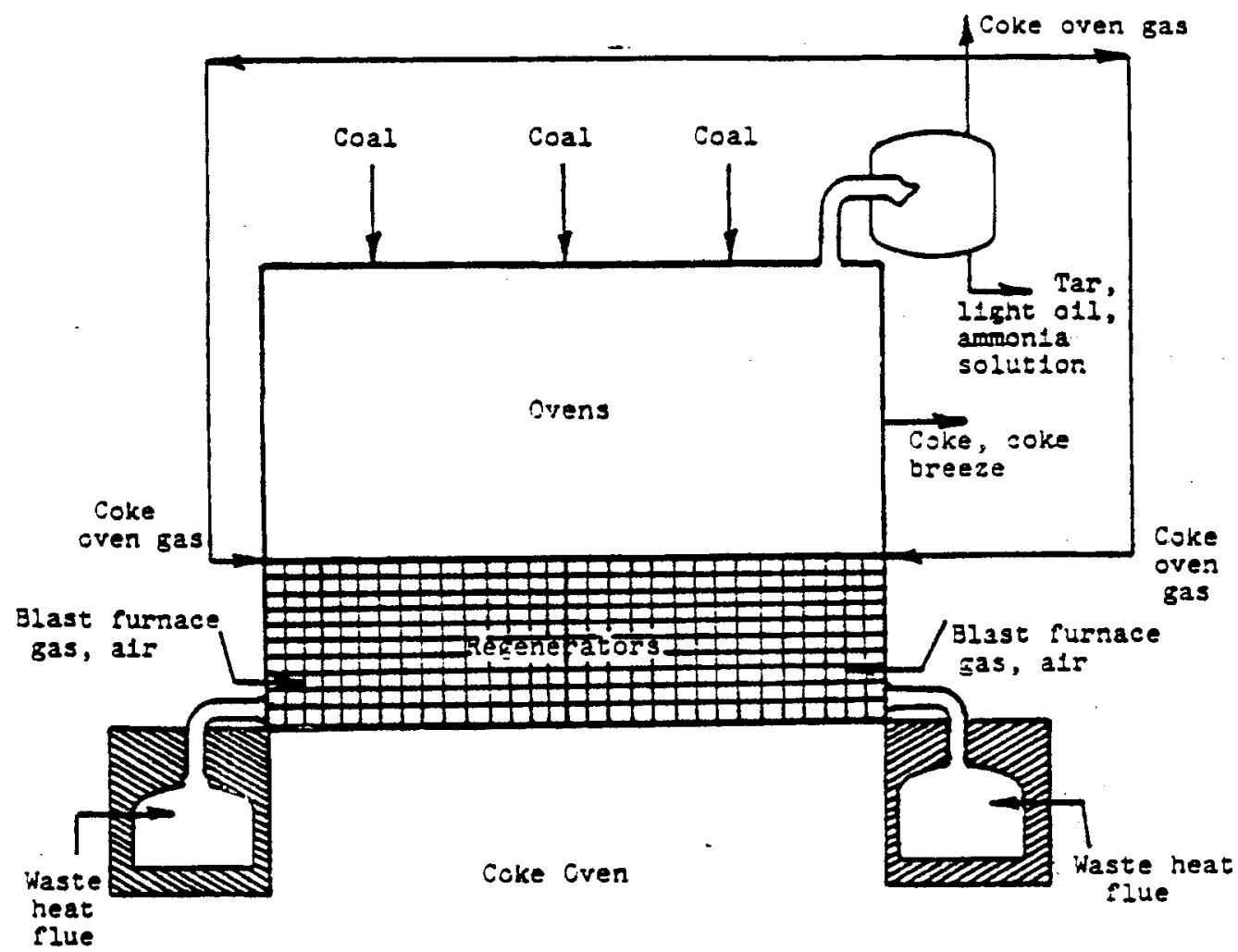

FIGURE C.2 Coke Oven (Reding et al. 1975)

An average-sized coke oven has a capacity on the order of $500 \mathrm{ft}^{3}$ of coal. With 50 or 60 ovens per battery, the throughput per battery would be in the range of 1200 tons per day. Energy consumption varies from 850 to 1050 Btu/pound of finished steel. This works out to approximately 1 MWt per oven. The thermal efficiency of coke ovens is close to $96 \%$, according to a report by Darby and Arons (1979).

\section{Agglomeration}

Agglomeration is the process of preparing iron-bearing materials for the blast furnace. The object is to form the iron bearing material into shapes and sizes that will be permeable to gas flow and yet not be blown out of the furnaces. Two processes are used for agglomerating, depending on the type of feedstock. Sintering occurs on a traveling grate in which iron ore fines have 
been mixed with coke breeze and limestone fines. The mixture is ignited at the front end of the grate and as it moves down the pathway, air is drawn past the grate to keep the coke burning. The heat from the burning sinters the mixtures into lumps that are more-or-less golf ball sized. The pelletizing operation is principally the same, except that pellets are first manufactured in a balling drum and coated with coal prior to being sent to the traveling grate. Sintering and pelletizing operations are depicted in Figures C.3 and C.4.

Larger sintering plants have capacities ranging up to 350 tons per hour; pelletizing plants are somewhat smaller. Energy consumption for these two operations runs between 600 and 700 Btu/pound of finished stee 1 .

\section{Blast Furnace}

The blast furnace is the central piece of equipment in an integrated steel mi11. In this operation, iron oxides are reduced to iron in the form of pig

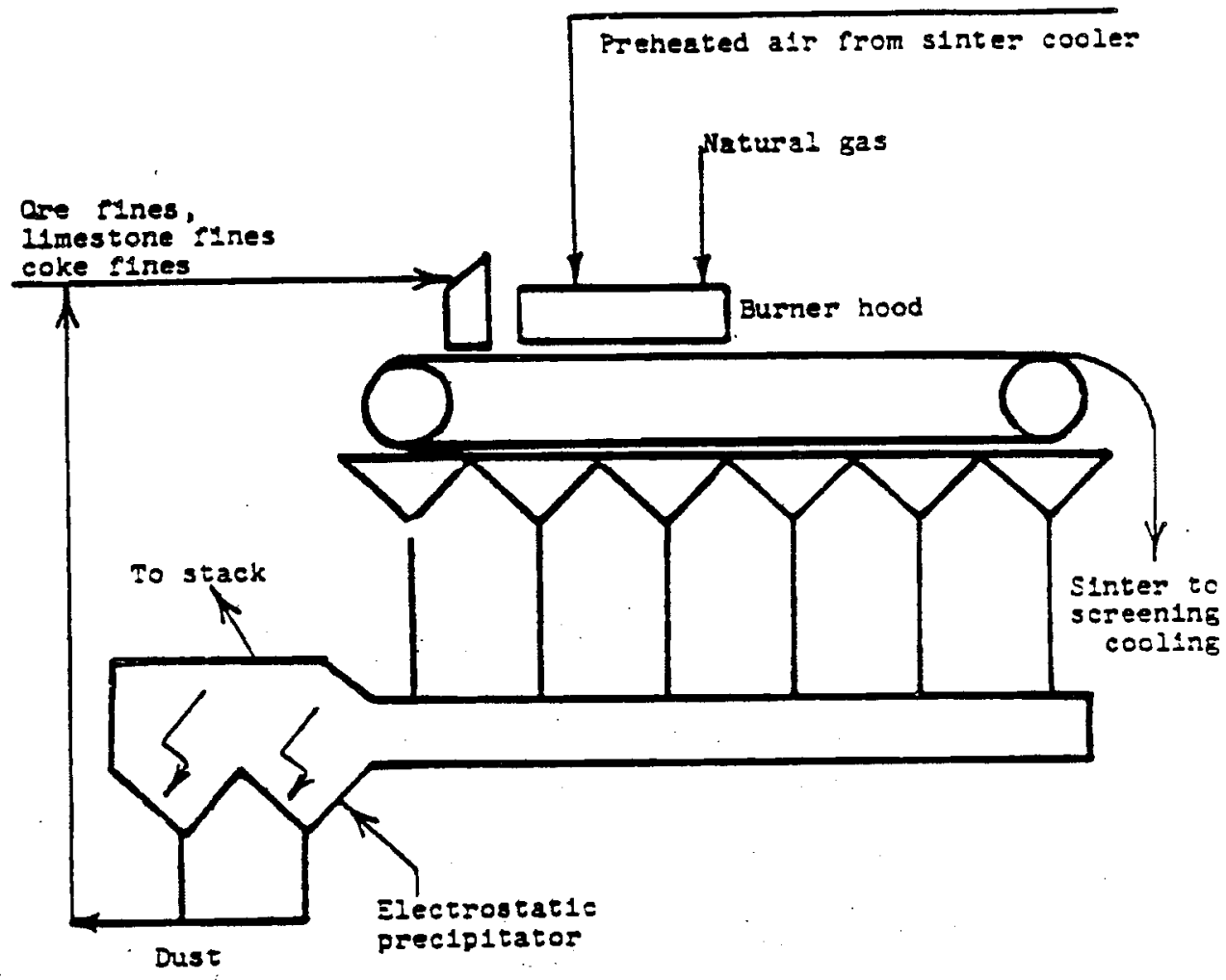

FIGURE C.3 Sintering Machine (Reding et a1. 1975) 

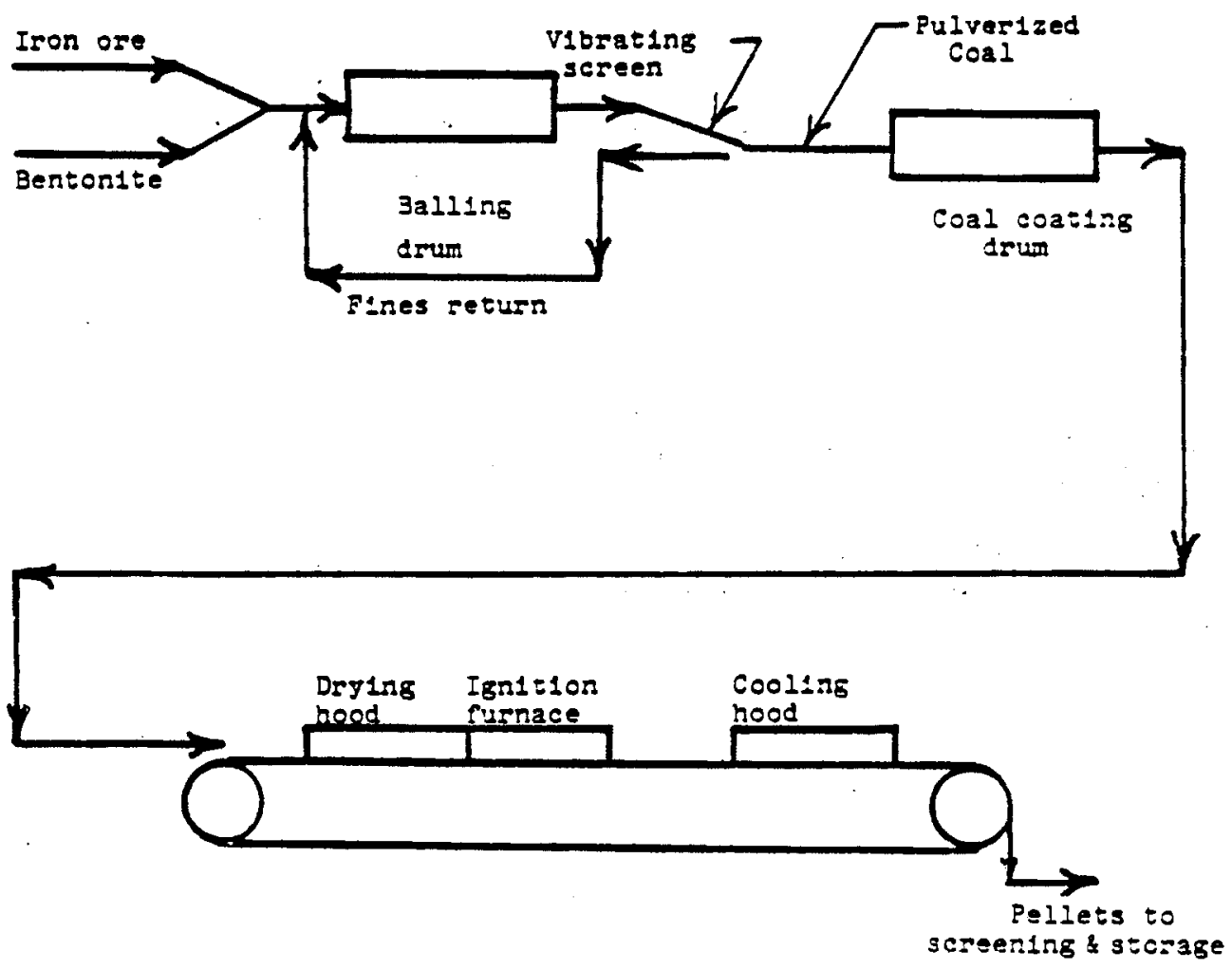

FIGURE C.4 Pelletizing Machine (Reding et al. 1975)

iron, which contains 4 to $5 \%$ carbon and other impurities. Iron ore, sinter, and pellets are charged to the top of a blast furnace along with coke, limestone, and smaller quantities of other fluxes. Hot air, which may often be enriched with oxygen, is injected near the bottom of the furnace through holes called tuyeres. The hottest portion of the furnace is at the bottom combustion zone where temperatures may reach $3000^{\circ} \mathrm{F}$. Hot combustion gases rise through the furnace to preheat recent charges near the top of the furnace. The temperature differential from top to bottom divides the furnace into several zones. As the preheated charge descends, it begins partial reduction when temperatures exceed $1000^{\circ} \mathrm{F}$. Direct reduction occurs when the temperature exceeds $2000^{\circ} \mathrm{F}$. As the charges descend to the bottom, where temperatures are near $3000^{\circ} \mathrm{F}$, the final reduction and slag-forming reactions occur. Molten iron and slag are then periodically tapped from the hearth. 
The blast furnace is a relatively large piece of equipment with a height ranging up to 100 feet and a diameter of 15 to 30 feet at the hearth. A drawing of a blast furnace and accompanying equipment is shown in Figure C.5. Modern blast furnaces typically have a production capacity varying aroung 100 tons of pig iron per hour. Approximately 0.60 tons of coke are consumed per ton of finished steel. Coke is both the reducing agent and the principal

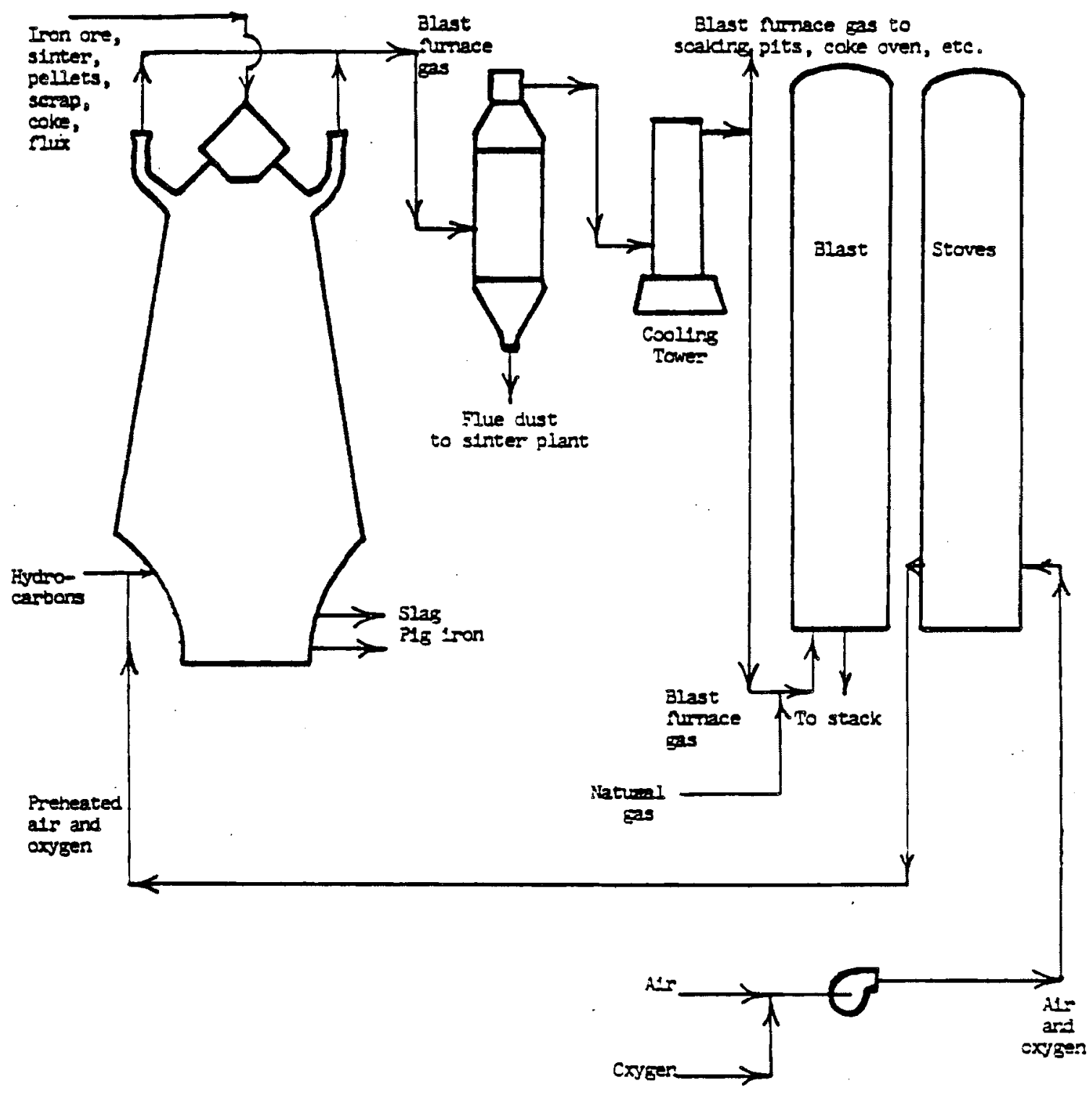

FIGURE C.5 Blast Furnace (Reding et al. 1975) 
source of thermal energy. Lesser amounts of other fuels and materials add between 1000 and $3000 \mathrm{Btu} / \mathrm{lb}$ of finished steel, depending on the operation and whether the energy content of pellets, sinter, ore, and limestone are included with the relatively small amounts of fuel oil, natural gas, coke oven gas, blast furnace gas, electricity, and steam that are consumed in this unit operation. A blast furnace requires these large quantities of energy to drive the endothermic reduction reaction and to melt the charge. The heat of reaction alone is 3000 Btu/lb of finished steel. The overall thermal efficiency of the blast furnace is reported by Darby and Arons (1979) to be $67 \%$.

\section{Steelmaking}

Although the open hearth furnace is declining somewhat in importance as a steelmaking process, it is the most significant in terms of energy consumption. In general, the pig iron produced from the blast furnace is high in carbon content and other impurities. The principal object of the steelmaking process is to remove these impurities and reduce the carbon content from 4 to $5 \%$ down to the range of $0.5 \%$, depending on the grade of carbon steel required. Pig iron, steel scrap, and fluxing agents are charged to the bath or hearth of the open hearth furnace and are heated from above by a flame. The fuel, usually either natural gas, fuel oil, or coke oven gas, and air are alternately injected from opposite sides of the furnace. The combustion products exit through a brick checker works that serves to preheat the fuel and air when flow is reversed. Although the impurities in the pig iron are oxidized by excess air or oxygen injections, the open hearth furnace is not designed to be energy self-sustaining. The prospects of lower energy consumption and higher production rates have driven the steel industry toward the basic oxygen furnace. Because the energy source is supplied externally, the open hearth furnace does have more flexibility in the feed mix, however. An open hearth furnace is shown in Figure C.6.

The open hearth furnace is a batch operation. Up to 10 hours are required per heat, depending on the temperature and chemical content of the charge. The typical furnace has a capacity of 200 tons (per batch) and consumes $1700 \mathrm{Btu} / 1 \mathrm{~b}$ of finished steel. 


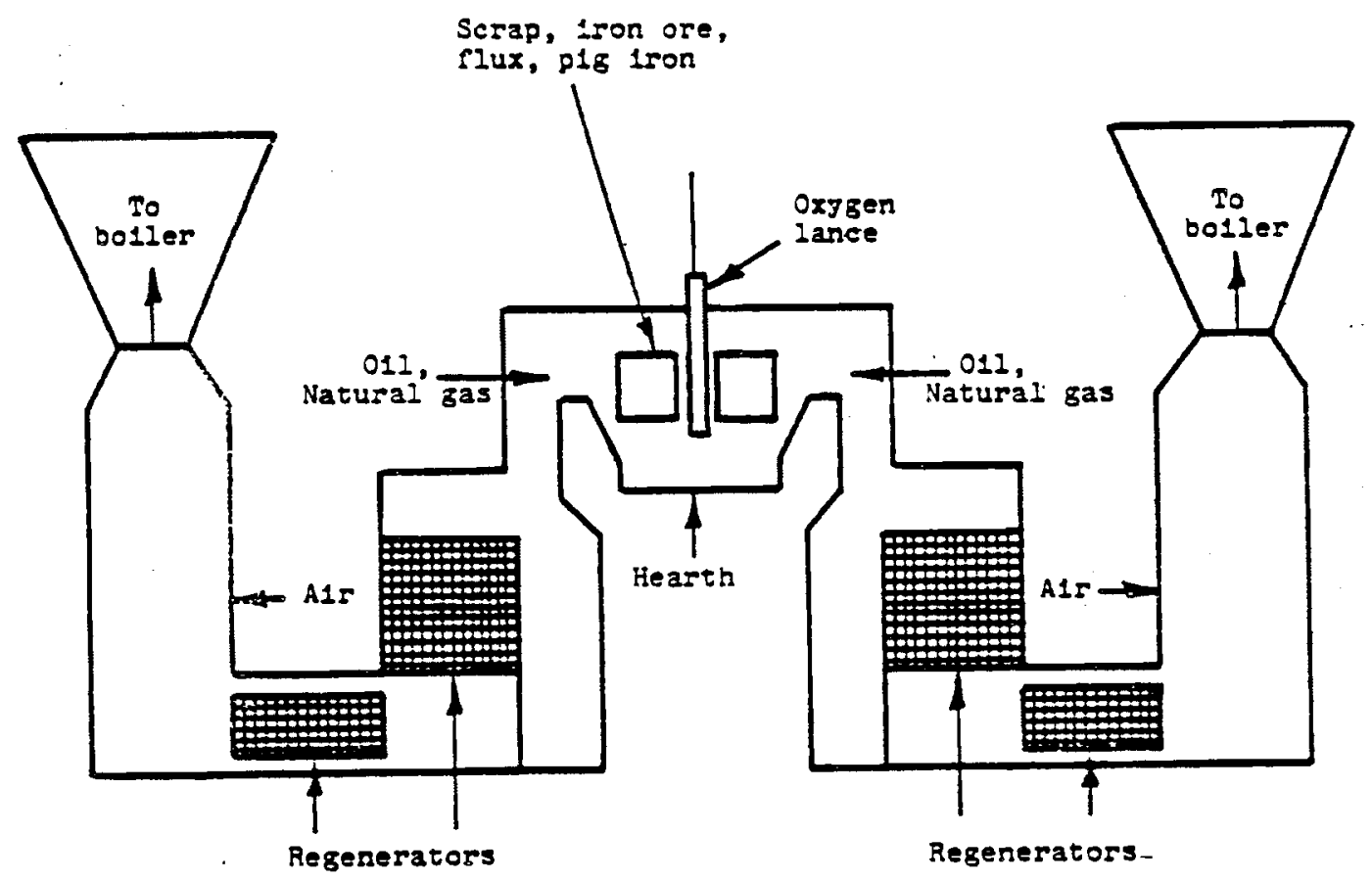

FIGURE C.6 Open Hearth Furnace (Reding et al 1 1975)

\section{Soaking Pits}

Unless continuous casting is used, steel ingots must be heated in a soaking pit prior to being rolled. Liquid steel from the steelmaking furnaces is most commonly poured into ingots and allowed to cool prior to production of semi-finished steel products. Continuous casting involves the direct production of semi-finished steel from liquid steel. This production path is more direct and consumes less energy, but is much more complicated. Steel ingots are heated in the soaking pits to between 2200 and $2500^{\circ} \mathrm{F}$. Ingots exiting the soaking pit should have achieved a uniform temperature and be soft enough for the rolling operations. A drawing of a soaking pit is shown in Figure C.7.

Soaking is a batch operation in which equipment size and heating rates vary drastically. Hearth areas range from 10 to $300 \mathrm{ft}^{2}$ and heating rates can be anywhere from 2 to $250 \mathrm{lb}$ per $\mathrm{ft}^{2}$ per hour according to U.S. Steel 


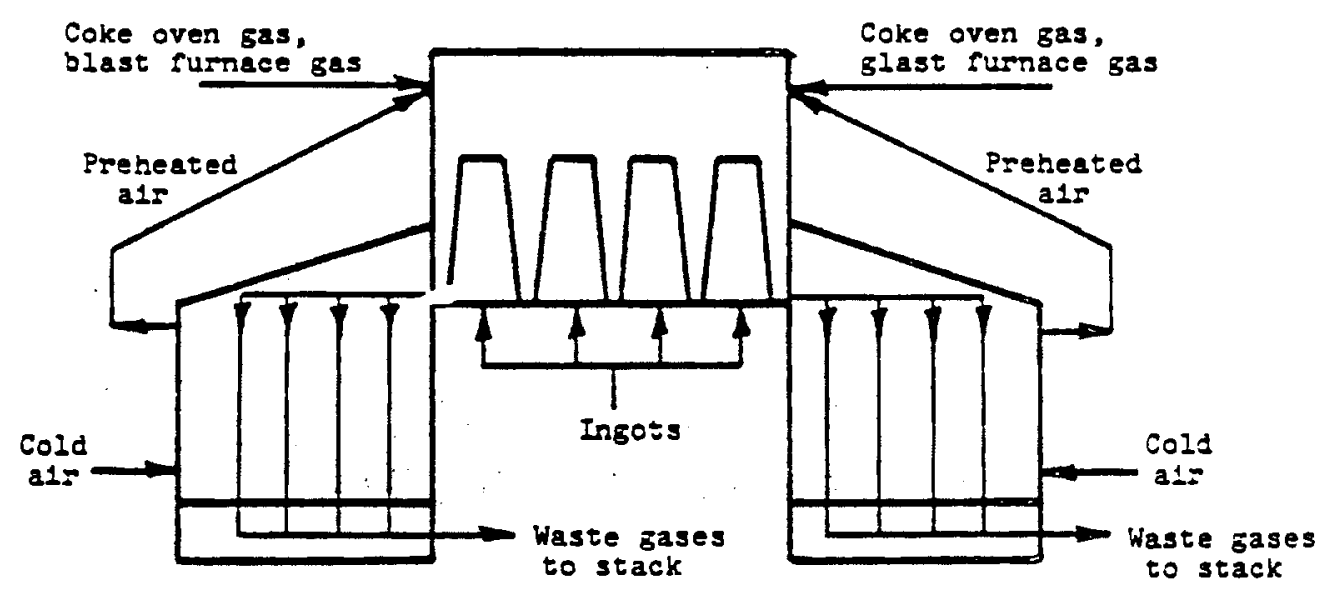

FIGURE C.7 Two-Way-Fired Soaking Pit (Reding et a1. 1975)

(1971). A typical soaking pit might process 5 tons of steel per hour. Soaking time can be as high as 8 to 12 hours for cold ingots. If ingots are charged hot, the time will obviously be shortened. Despite the large number of factors affecting soaking pit performance, several sources ( $B C L$ 1975; Reding et al.; 1975; Darby and Arons 1979) indicate that energy consumption in the soaking pit is close to 750 Btu/1b of finished steel.

\section{Reheating Furnaces}

After leaving the soaking pits, ingots are rolled into semi-finished products called blooms, slabs, and billets. At this point in the steel mill, the semi-finished products are reheated so they can be rolled into finished products such as pipes, plates, sheets, and structural steel. As opposed to the soaking furnaces, reheat furnaces are usually designed for continuous operation and have a much higher throughput per hour. The heating ratio depends on the number of heating zones; $100 \mathrm{lb} / \mathrm{hr}-\mathrm{ft}^{2}$ of hearth and $150 \mathrm{lb} / \mathrm{hr} \mathrm{ft}^{2}$ of hearth are common for two- and three-zone furnaces, respectively. Continuous reheating furnaces range in size from 50 to $250 \mathrm{ton} / \mathrm{hr}$. Those reheat furnaces that are batch oriented have capacities in the range of 5 to $25 \mathrm{ton} / \mathrm{hr}$. Energy requirements average between 1400 and 1500 Btu/lb of finished steel product. According to Arons and Darby (1979) reheat furnaces are only 25 to $30 \%$ 
thermally efficient, despite employing some recuperation. A typical reheat furnace is shown in Figure C.8.

\section{Annealing Furnaces}

A portion of the products leaving a steel mill are heat treated in an annealing furnace to relieve stresses within the metal. Annealing furnaces have historically been batch-oriented, but continuous furnaces are occasionally employed. Annealing occurs at temperatures in the range of 1200 to $1500^{\circ} \mathrm{F}$ as opposed to the higher temperatures associated with soaking and reheating. Batch periods may run as long as 1 to 2 days with the charge varying from 40 to 400 tons. Continuous furnaces operate at production levels of 5 to 15 tons per hour. Reported fuel consumption varies between 300 and 1000 Btu/1b of finished steel. As was the case for reheat furnaces, annealing furnaces are not thermally efficient. A value of $30 \%$ is reported in Industrial Heating (1981). Figure C.9 depicts an annealing furnace for coiled strip.

\section{Waste Energy Streams}

In an industry that consumes as much energy as the steel industry it is not surprising that enormous quantities of energy are rejected that could

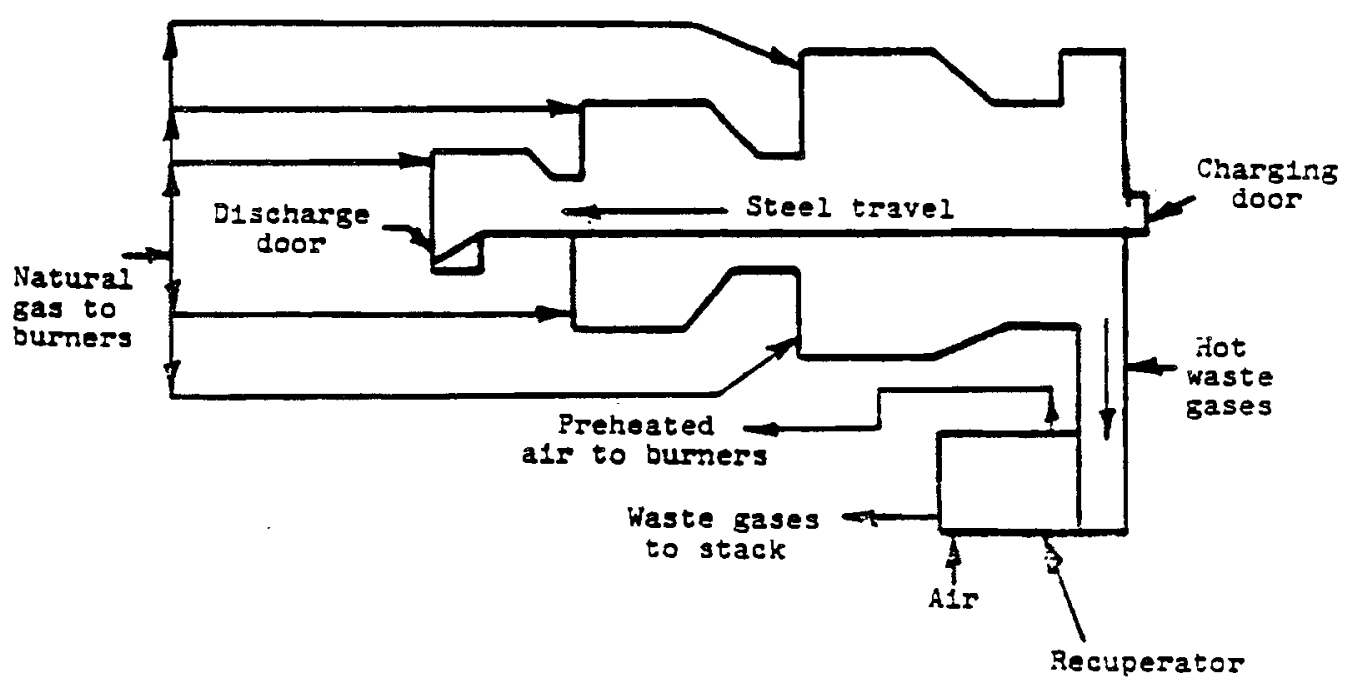

FIGURE C.8 Reheating Furnace (Reding et al. 1975) 


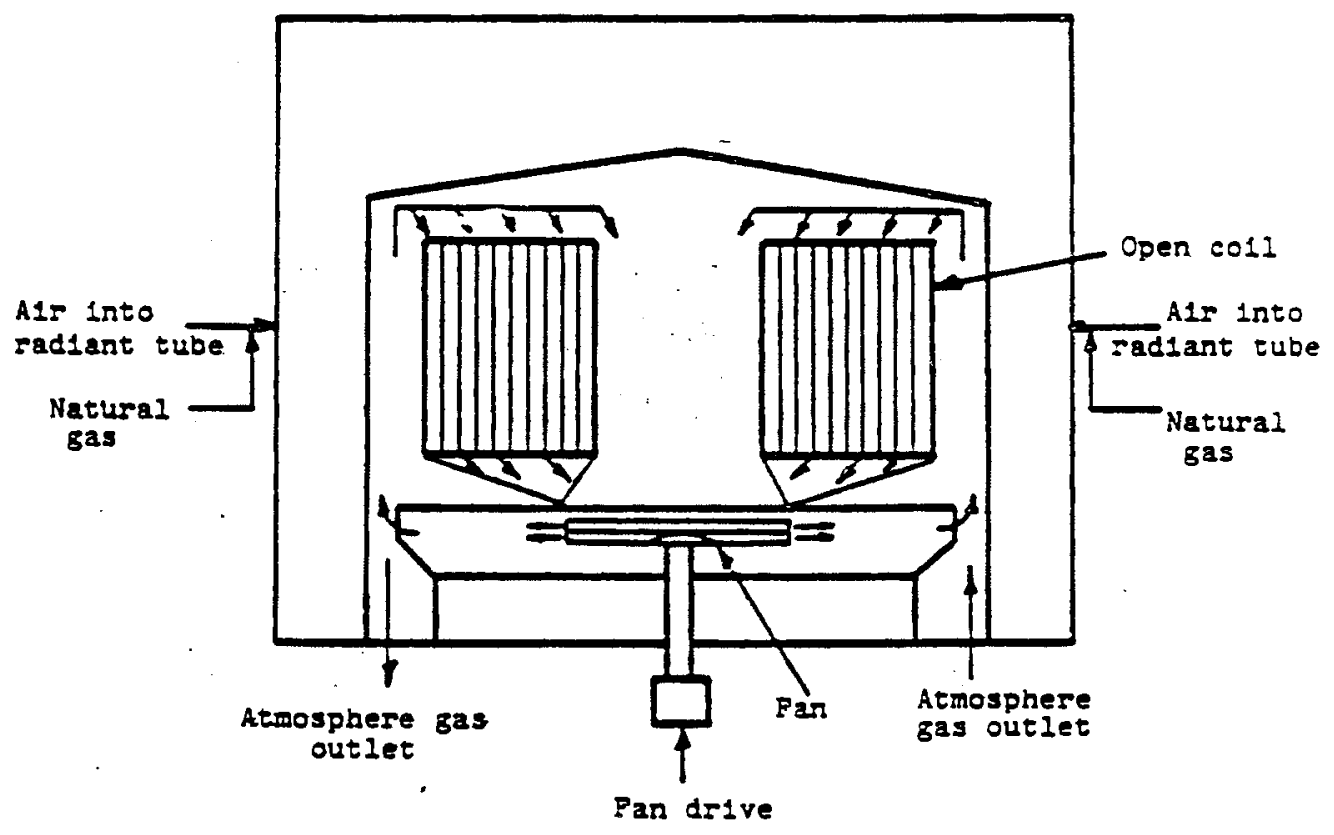

FIGURE C.9 Annealing Furnace For Coiled Strip (Reding et al. 1975)

potentially be recovered. A lot of this energy is in the form of flue gases from the various types of furnaces described in the previous section. An equal amount of energy is rejected to cooling water streams that circulate through the high-temperature furnaces. Waste energy streams are described for individual unit operations and the industry as a whole in the following subsections.

\section{Coking}

The steel industry does a good job of capturing coke oven gas for consumption at the coking plant or in other plant operations. Only relatively small amounts of coke, coke oven gas, and other by-products are lost or unrecovered. The coke oven flue gas $\left(700^{\circ} \mathrm{F}, 300 \mathrm{Btu} / \mathrm{lb}\right.$ of finished steel) offers some potential for heat recovery. The greatest potential for waste heat recovery in the coking plant lies with the quenching of coke. Currently, coke is quenched with water after it is discharged from the oven. The energy is dissipated to the environment without an attempt to recover it. Alternatively, the coke could be quenched with a cool, inert gas in a process called dry quenching. Heat recovery from the inert gas could yield 900 to 1000 lbs of high pressure steam per ton of finished steel. 


\section{Agglomerating}

Additional waste heat recovery from the sintering and pelletizing operations does not appear to be attractive. Although the exhaust gases reject 400 to 500 Btu per pound of steel product, the flue gas temperatures are only in the range of 250 to $300^{\circ} \mathrm{F}$.

\section{Blast Furnace}

Industry currently does a good job of collecting the blast furnace gases produced from this operation, but could potentially do better by capturing the thermal as well as chemical content (100 Btu/scf) of the off-gas. Blast furnace gas and associated dust comes off the top of the blast furnace at temperatures in excess of $500^{\circ} \mathrm{F}$. The gas is currently cleaned and cooled prior to being distributed for consumption around the plant. Recovery of this thermal energy may be possible if abrasive-resistant equipment can be employed. Another candidate for waste heat recovery at the blast furnace is the molten slag that is periodically tapped from the furnace. This slag has a temperature approaching $3000^{\circ} \mathrm{F}$ with an energy flow rate of $250 \mathrm{Btu} / \mathrm{lb}$ of finished steel.

\section{Basic 0xygen Furnace}

Many reports identify the basic oxygen furnaces (BOF) off-gases as a candidate for waste heat recovery (A.D. Little 1975; BCL 1975; Palmer et al. 1980; Reding et a1. 1975). The gas comes off the BOF at about $2300^{\circ} \mathrm{F}$ and has an energy content of $350 \mathrm{Btu} / \mathrm{scf}$ at that point and 200 to $250 \mathrm{Btu} / \mathrm{scf}$ after collecting and cooling. Approximately 150 to 250 Btu/lb of finished steel could be recovered. The erosive nature of the particulates contained in the off-gas may inhibit thermal energy recovery. The composition of the particulates in the gas stream is identified in Table C.2.

Soaking, Reheating, and Annealing Furnaces

Waste heat recovery in soaking, reheating, and annealing furnaces has received much attention. The relatively low thermal efficiencies (25 to $30 \%$ ) identified in the previous section for these operations points out the need for 


\section{TABLE C.2 BOF Off-Gas Dust Composition}

\begin{tabular}{lrr}
\cline { 1 - 1 } Component & & Weight \% \\
\cline { 1 - 1 } $\mathrm{Fe}^{2} 0$ & & 1.6 \\
$\mathrm{Fe}_{3} \mathrm{O}_{4}$, & & 57.4 \\
$\mathrm{Fe}_{2} \mathrm{O}_{3}$ & & \\
$\mathrm{CaO}$ & & 2.0 \\
$\mathrm{SiO}_{2}$ & & 1.0 \\
Other & & 38.0
\end{tabular}

advanced waste heat recovery techniques. Ruskin and Bonner (1979) estimated up to $300 \mathrm{Btu} / \mathrm{lb}$ of finished steel could be recuperated from soaking pits through the use of advanced equipment. Darby and Arons (1979) reported that $10 \%$ of steel soaking pits had no recuperators. Ceramic materials are expected to enhance the recuperative capabilities in the future.

According to Gerlaugh et al. (1979), the potential for applying advanced energy recovery techniques also applies to reheating furnaces where only onehalf of the furnaces had recuperators. Here the energy recovery potential was estimated to be $400 \mathrm{Btu} / \mathrm{lb}$ of finished steel. An additional $500 \mathrm{Btu} / \mathrm{lb}$ of steel is rejected to the cooling water in the furnace skid pipes.

Waste Heat in Process and Cooling Waters

The waste heat recovery potential in process and cooling water streams is often overlooked because of extremely low energy density. Although the energy density is low, the quantities are immense. Almost all unit operations in a steel mill use water for either processing or cooling. The energy availability for process and cooling water streams is identified in Tables C.3 and C.4.

Estimated Waste Heat Discharges by Integrated Steel Mills

A report by Latour and Menningman (1981) has identified the sources, quality, and quantity of waste heat discharges in the energy-intensive industries. The data presented in their report identify the extremely large quantity of waste heat that is rejected by the iron and steel industry. Equal amounts of 
TABLE C.3 Energy Content in Process Waste Water Streams (Latour and Menningman 1981)

$\begin{array}{lc}\text { Unit Operation(s) } & 10^{6} \text { Btu/Ton of Steel } \\ \begin{array}{l}\text { Blast furnace \& } \\ \text { sintering }\end{array} & 0.85 \\ \text { Vacuum degassing } & 0.17 \\ \text { Continuous casting } & 0.21\end{array}$

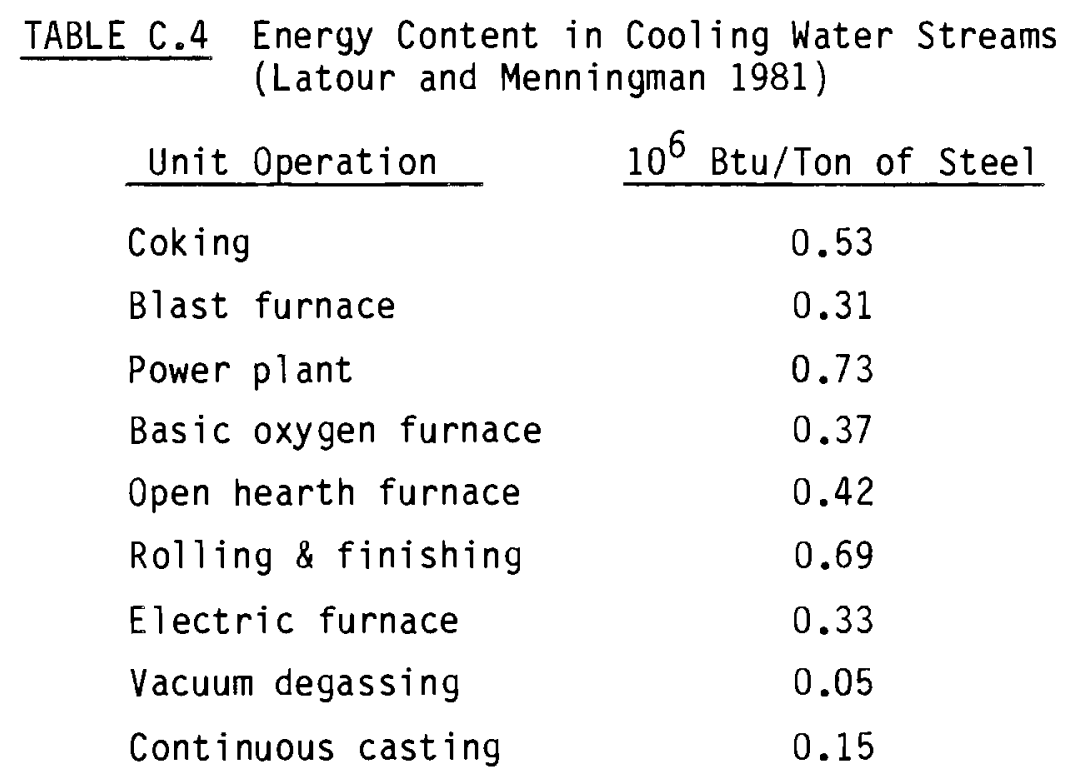

waste energy in the form of gases and water combine to a total of 1.4 quads on an annual basis. The report also points out that large amounts of waste heat are contained in process and cooling water streams. Most of these water streams have temperatures in the range of 100 to $125^{\circ} \mathrm{F}$. If heat pumps or some other innovative technology could take advantage of these low energy density sources, the potential for waste heat recovery would be enormous. Table C.5 identifies the quantity of waste heat in the blast furnace and steel mill industry according to several temperature ranges. 
TABLE C.5 Quantity and Quality of Waste Heat in the Blast Furnace and Steel Mill Industry (Latour and Menningman 1981)

\begin{tabular}{|c|c|}
\hline Temperature Range & $10^{12} \mathrm{Btu}$ \\
\hline 125 & 706 \\
\hline $125-500$ & 294 \\
\hline $500-1000$ & 286 \\
\hline $1000-1500$ & 77 \\
\hline $1500-2000$ & 47 \\
\hline 2000 & 9 \\
\hline
\end{tabular}

GRAY IRON FOUNDRIES

The gray iron foundry industry is relatively diffuse when compared to other industries within the primary metals sector. As of 1977, 984 establishments were engaged in some activity related to the production of gray iron products (1977 Census of Manufactures). Almost 300 of these had 100 or more employees, which together accounted for approximately $90 \%$ of the annual production of $13 \mathrm{million}$ tons. In recent years production has fluctuated between 10 and 15 million tons per year. As is the case for most primary metal industries, a gray iron foundry has several high temperature, energy-consuming operations. This equipment and its energy charges and discharges will be discussed in the following subsections.

Process Description

The central piece of equipment in a gray iron foundry is the furnace, which can either be a cupola, electric induction, or electric arc type. The furnace charge consists of iron scrap, pig iron, flux, and other metallic scrap. Other major operations include casting, mold making, and cleaning and finishing. Some products are heat treated as a final processing step. These steps are shown in Figure C.10.

Purchased scrap iron is the major source of raw material for gray iron foundries; other sources include pig iron, scrap steel, and various ferroalloys (United Technologies Corporation 1980). About $75 \%$ of the metal feedstock is 


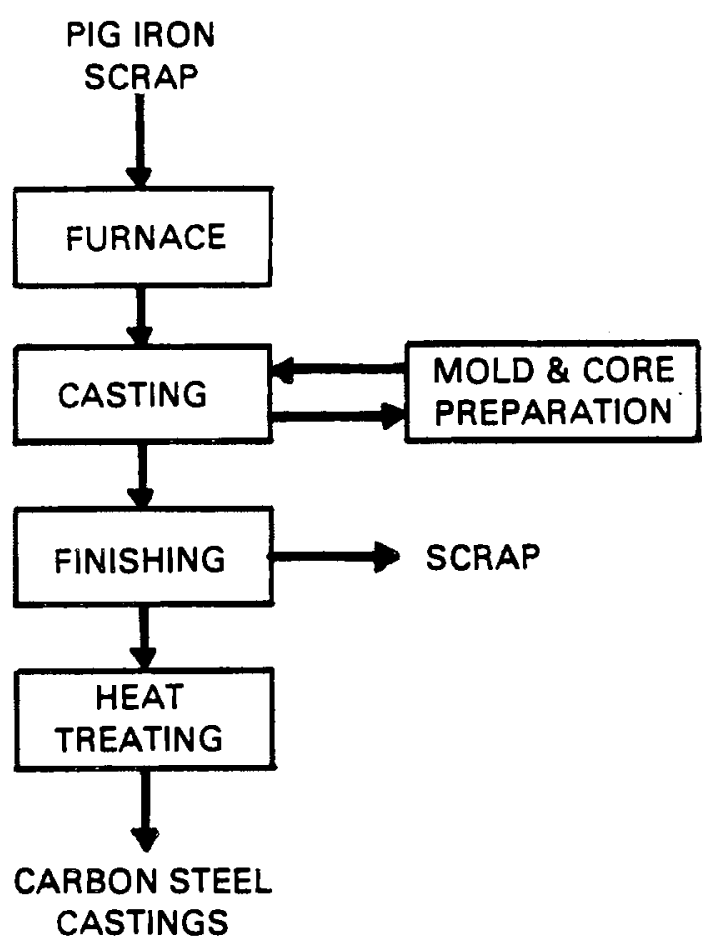

FIGURE C.10 Major Iron Foundry Operations

melted in cupola furnaces, with the balance melted in either the elective induction or electric arc furnaces. Electric furnaces are usually employed where higher quality castings are required because product composition is more easily controlled than in a cupola. Malleable iron castings are almost always melted electrically. Castings are produced by pouring or injecting molten iron into a mold. After the casting has cooled, it is removed from the mold and cleaned by sandblasting, scraping, and other finishing operations. The cast product may then be heat treated prior to shipment. According to Darby and Arons (1979), $10 \%$ of the gray iron, $50 \%$ of the ductile iron, and all of the malleable iron require heat treating. The molds are manufactured from silica sand, which is bonded together by binding materials such as bentonite or sodium silicates. Mold preparation is a major activity in a foundry. Heat Transfer Operations

The iron melting furnaces are the dominating heat transfer operations in a gray iron foundry. This is the most energy-intensive unit operation in a 
foundry and accounts for about half of the total energy consumption. Approximately $75 \%$ of the steel is melted in cupolas and the balance is done in electric furnaces. Both of these directly heating operations are discussed in more detail below.

\section{Cupola Furnace}

The cupola is a vertical cylindrical steel furnace lined with refractory. This is the same type of furnace that is used for reducing iron ore to pig iron in the steel industry, only smaller. Pig iron, scrap iron, and other iron alloys are charged with coke, limestone, and other fluxing agents at the top of the furnace. Hot air is blown in at the bottom of the furnace through holes called tuyeres. Combustion of the coke provides the energy for melting the charge. Liquid metal is withdrawn near the bottom of the furnace at temperatures of about $2700^{\circ} \mathrm{F}$.

Cupola furnaces in a gray iron foundry typically are sized to melt 10 tons of steel per hour as compared to 100 tons per hour for a blast furnace. Cupola melters range in height up to 50 feet with diameters typically 8 to 12 feet. Coke provides the majority of the energy for melting. Natural gas or fuel oil may also be injected through the tuyeres to provide an additional energy source. The energy consumed for melting is about $4 \times 10^{6} \mathrm{Btu} / \mathrm{ton}$. Because about one-half of the castings are scrapped and returned to the furnace, the energy consumed per net product is about $8 \times 10^{6} \mathrm{Btu} / \mathrm{ton}$.

\section{Electric Furnaces}

Electric furnaces are either the induction or arcing type, with the former being preferred in the gray iron foundry industry. An induction furnace supplies heat to the charge by changing the electrical flux in an induction coil that wraps around the furnace shell. When an alternating current is supplied to the coil, a rapidly changing magnetic field is set up that causes secondary current to form in the metal charge. The resistance of the metal converts the electric current to heat and melts the charge. In an electric arc furnace, heat is generated as current flows through the air gaps between carbon electrodes and the metal. 
Electric furnaces are more efficient than cupolas, al though they obviously consume a more costly energy source. Energy consumption for an electric furnace is about $2 \times 10^{6} \mathrm{Btu} / \mathrm{ton}$ melted. Again, this figure would double if the energy basis were per ton of cast product.

Waste Heat Streams

Relatively little is mentioned in the literature regarding waste heat recovery potential in the gray iron foundry industry. The industry could benefit most by reducing the percentage of castings which must be recycled. There appear to be opportunities for better management of flue gases from the melting furnaces and various other operations.

According to Latour and Menningmann (1981), the single largest source of waste heat in a gray iron foundry is the flue gas emanating from the scrap preparation process. $0 i 1$ must be removed from scrap by heating it. The amount of waste heat from this operation was estimated to be nearly $31 \times 10^{12}$ Btu per year. This represents $60 \%$ of the total flue gas waste heat being discharged.

The waste heat contained in furnace cooling waters and flue gas scrubbing waters was also estimated by Latour and Menningmann (1981). These estimates are summarized in Table C.6. Although cooling waters and other process water streams contain substantial amounts of waste heat, their low temperatures $\left(125^{\circ} \mathrm{F}\right)$ make energy recovery difficult. A summary of the total waste heat discharged by gray iron foundries is given in Table C.7.

\section{PRIMARY COPPER}

Primary copper is one of the most heavily concentrated industries in the primary metals or any other sector. Only 27 establishments were involved in

TABLE C.6 Water Source Waste Heat in Melting Furnaces

\begin{tabular}{lc}
\multicolumn{1}{c}{ Flow Stream } & $10^{6}$ Btu/ton Melted \\
$\begin{array}{l}\text { Cupola cooling } \\
\text { Electric furnace } \\
\text { cooling }\end{array}$ & .31 \\
Flue gas scrubbing & .39 \\
& .67
\end{tabular}



TABLE C.7 Quantity and Quality of Waste Heat in
the Gray Iron Foundry Industry

\begin{tabular}{cc} 
Temperature Range & $10^{12}$ Btu \\
\hline$<125$ & 22 \\
$125-500$ & 21 \\
$500-1000$ & 12 \\
$1000-1500$ & 8 \\
$1500-2000$ & 7 \\
$>2000$ & 1
\end{tabular}

primary copper production in 1977 according to the Census of Manufactures. Most of these were located in the Southwest. Copper production in a typical year will be approximately 2 million short tons; production in 1981 was 1.7 million tons according to the 1983 Mineral Commodity Summaries and had fallen to 1.3 million tons in recessionary 1982. Copper production is highly concentrated due to strong economies-of-scale in smelter operations. Plant capacities in the range of 100,000 tons per year are typical for the industry. High-temperature, high-energy flux operations are the rule rather than the exception in the primary copper process. These unit operations ana their energy streams are discussed in the following subsections.

Process Description

Copper smelting and refining consist of several consecutive unit operations that remove the noncopper impurities from the feedstock as it moves through the plant. Concentrated ore, either wet and/or partially dried in a roaster, is fed to a reverberatory smelting furnace. Copper and ferrous sulfides collect at the bottom of the furnace; slag is removed from the top. Flue gases from the smelting furnace are passed through a waste-heat boiler prior to cleaning. The matte (copper and ferrous sulfides) is transferred next to the converter. Iron and sulfur are oxidized in the converter with a high silica flux and forced air. Slag from this operation is recycled to the smelter. The product from the converter is called blister copper, which is about 98\% pure. Flue gases from the converter are cleaned and sent to a sulfuric acid plant. 
The final processing steps involve fire refining and/or electrolytic refining to achieve the desired copper purity. These steps are shown in figure C.11. Heat Transfer Operations

The smelting furnace is the most important heat-transfer operation in a primary copper plant. Other significant heat-transfer operations to be discussed in the following subsections include the fire refining furnace, electrolytic refining furnace, and cathode melting.

\section{Smelting Furnace}

The most energy-intensive unit operation in a primary copper plant is the smelting furnace. Concentrated copper ores are fed to a reverberatory furnace with fluxing agents. The ores may be fed green (wet), dried, or calcined. The fluxing agents combine with the gangue material to form a slay while copper and

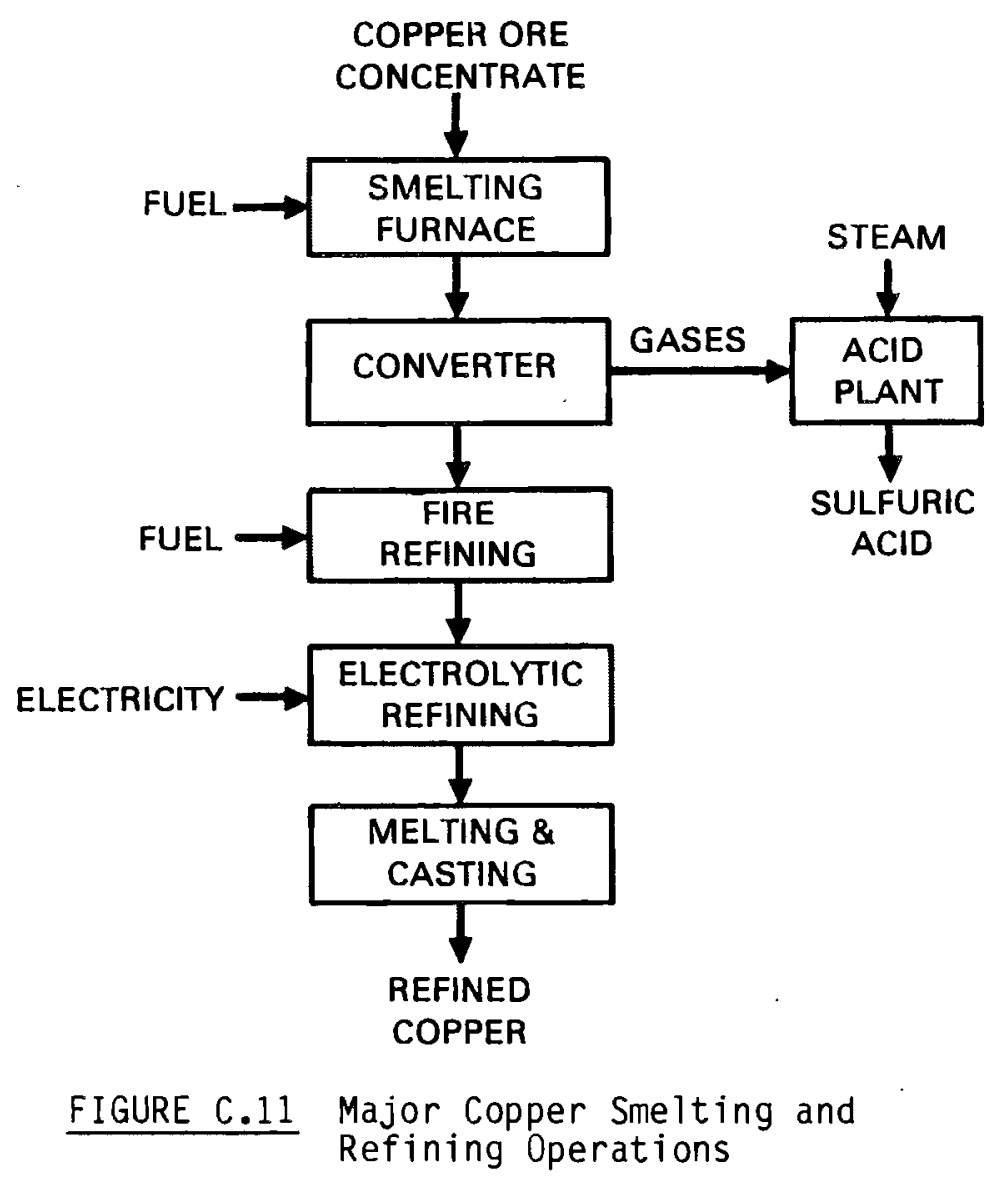


ferrous sulfides collect at the bottom of the furnace in a form known as matte. A portion of the sulphur content is oxidized to $\mathrm{SO}_{2}$ and exits with the flue gas. Energy consumption will obviously vary depending on whether the feed has been dried or calcined. Several sources have estimated the energy requirements for this operation to be about $24 \times 10^{6} \mathrm{Btu} / \mathrm{ton}$ of refined copper (Bodine and Vitullo 1980; A.D. Little 1976; Hamell et al. 1979). Sources reporting lower figures of 14 to $15 \times 10^{6}$ Btu/ton have probably al ready taken credit for energy recovery from the flue gas.

The smelting furnace operates at temperatures close to $2200^{\circ} \mathrm{F}$ (Bodine and Vitullo 1980; Gaines et al. 1980; Hamell et al. 1980), although Hall (1974) reports temperatures as high as $3000^{\circ} \mathrm{F}$. As was mentioned in the introduction, the copper industry is highly concentrated, with individual smelters having capacities up to 1500 tons/day (Hall 1974). Palmer et al. (1980) reports capacities of 450 tons/day and 200 tons/day for "medium" and "smal1" sized smelters.

Fire Refining (anode furnace)

The iron and copper sulphides in the matte from the smelting furnace are oxidized in the converter. The product from that operation is called blister copper, which is approximately 98\% pure. Further refining occurs in this step in an operation similar to open hearth steelmaking. Impurities within the blister copper are preferentially oxidized when submitted to the $2100^{\circ} \mathrm{F}$ conditions of the reverberatory fire refining furnace. Reported energy consumption for this operation varies from 3.5 to $3.710^{6}$ Btu/ton of refined copper (Bodine and Vitullo 1980; Gaines et al. 1980; Hamell et al. 1980). Furnace capacity ranges fron 165 to 330 tons for this batch operation.

\section{Electrolytic Refining and Cathode Melting}

Depending on the requirements for end-product consumption, the copper purity resulting from fire refining may be adequate. If not, the copper is further refined by electrolytic means. Electrolytic refining occurs in a solution of copper sulphate and sulphuric acid. Fire refined copper is used as the 
anode and pure copper plates out at the cathode. The cathodes are then melted and cast into more useful shapes. Electrolytic refining requires both electric energy and thermal energy for heating the electrolytic solution. Reported energy consumption for these three unit operations vary widely in the literature. Energy consumption estimates are compared in Table C.8.

Waste Heat Streams

Waste heat recovery potential in a primary copper plant is dominated by the smelting furnace. Because the smelter is the largest energy consumer, it has the greatest potential for heat recovery. The flue gas exits the smelter at about $2250^{\circ} \mathrm{F}$ and carries with it $14.2 \times 10^{6} \mathrm{Btu} / \mathrm{ton}$ of refined copper (Hammel et al. 1980). The majority of this energy is recovered in waste heat boilers; Bodine and Vitullo (1980) has estimated the energy recovery from flue gases to be $9.35 \times 10^{6}$ Btu/ton of copper. An impediment to waste heat recovery is the sulphur dioxide content of the flue gas. The portion of flue gas thermal energy that is not currently recovered could possible be used to preheat the concentrated ones. Concentrations (by volume) of 0.5 to $1 \% \mathrm{SO}_{2}$ for calcined charges and 1.5 to $3 \% \mathrm{SO}_{2}$ for green charges are reported by Gaines et a1. (1980). Smelter slag is another waste heat source that is currently unrecovered. The energy contained in the slag is estimated by Hammel et

TABLE C.8 Reported Energy Consumption for Electrolytic Refining and Cathode Melting

\begin{tabular}{|c|c|c|}
\hline Operation & Energy Consumption & Source \\
\hline Refining-electric (a) & $\begin{array}{l}3.23 \times 10^{6} \text { Btu/ton } \\
2.63 \times 10^{6} \text { Btu/ton } \\
2.93 \times 10^{6} \text { Btu/ton }\end{array}$ & $\begin{array}{l}\text { Bodine and Vitul10 } 1980 \\
\text { A.D. Little } 1976 \\
\text { Hamell et al. } 1980\end{array}$ \\
\hline Refining-thermal & $\begin{array}{l}4.2 \times 10^{6} \text { Btu/ton } \\
2.0 \times 10^{6} \text { Btu/ton } \\
0.3 \times 10^{6} \text { Btu/ton }\end{array}$ & $\begin{array}{l}\text { Bodine and Vitullo } 1980 \\
\text { A.D. Little } 1976 \\
\text { Hamell et al. } 1980\end{array}$ \\
\hline Cathode melting & $\begin{array}{l}1.95 \times 10^{6} \text { Btu/ton } \\
3.0 \times 10^{6} \text { Btu/ton } \\
4.0 \times 10^{6} \text { Btu/ton }\end{array}$ & $\begin{array}{l}\text { Bodine and Vitullo } 1980 \\
\text { Hamell et al. } 1980 \\
\text { Hall } 1974\end{array}$ \\
\hline
\end{tabular}

(a) Based on 10,500 Btu/kWh 
a). (1980) to be $3.4 \times 10^{6}$ Btu/ton of copper. Flue gases from fire refining and cathode melting are also potential sources of recoverable energy. The current rejection of flue gases is $3 \times 10^{6} \mathrm{Btu} / \mathrm{ton}$ of copper, which exit these operations at temperatures of about $2600^{\circ} \mathrm{F}$ (Hammel et al. 1980).

Primary copper plants are similar to other high-temperature metals processes in that significant amounts of non-contact cooling water are required to cool the high-temperature furnaces. The waste heat contained in contact and non-contact cooling waters have been estimated by Latour and Menningman (1981). These estimates are summarized in Table C.9. Latour and Menningman (1981) also estimated the energy discharged from primary copper plants in the form of flue gases. Their estimates for primary copper waste heat discharges are summarized in Table C.10 according to temperature.

\section{PRIMARY ALUMINUM}

The primary aluminum industry, like primary copper, is highly concentrated. According to Bodine (1980), there are 12 primary aluminum producers in

TABLE C.9 Energy Contained in Primary Copper Plant Water Streams (Latour and Menningman 1981)

\begin{tabular}{lr} 
Unit Operation & Btu/ton of Copp \\
\hline Smelting & $1 \times 10^{6}$ \\
Acid plant & $2 \times 10^{6}$ \\
Fire refining & $1 \times 10^{6}$ \\
Misc. &
\end{tabular}

TABLE C.10 Quantity and Quality of Waste Heat Discharged in the Primary Copper Industry (Latour and Menningmann 1981)

\begin{tabular}{|c|c|}
\hline Temperature Range $\left({ }^{\circ} \mathrm{F}\right)$ & $10^{12} \mathrm{~B}$ \\
\hline 125 & 21 \\
\hline $125-500$ & 34 \\
\hline $500-1000$ & 21 \\
\hline 1000 & -- \\
\hline
\end{tabular}


the U.S. operating 31 reductions plants. Individual plants vary in capacity from 36,000 to 285,000 tons per year. Total production capacity is about 5,000,000 tons/year. Actual production was near this level in 1981 but has dropped off due to the recent (1982) recession. Because aluminum production is extremely energy intensive and requires electricity for the reduction process, the industry is principally located where energy is cheap and/or abundant. The majority of plants are located in the Pacific Northwest, the Gulf Coast, Tennessee and Ohio River Valleys, and the St. Lawrence Seaway.

Process Description

Activity at an aluminum reduction plant centers on the potlines where alumina $\left(\mathrm{Al}_{2} \mathrm{O}_{3}\right)$ is electrolytically reduced to aluminum. Alumina is charged to the reduction cell, where it is first dissolved in a solution of molten cryolite. The cryolite solution serves as an electrolyte. A high current passes through the solution from the carbon anode to the cathode, where aluminum is deposited. 0xygen liberated in the process collects at the anode and combines with the carbon surface to form $\mathrm{CO}_{2}$.

The carbon anodes are prepared for reduction cells onsite, either by the pre-baked or Soderberg process. High-grade petroleum coke and pitch is ground, calcined, and blended into a paste-like mixture that is sent directly to the reduction cell in Soderberg operations. The molding and baking of the paste occurs in the reduction cell for the Soderberg systems and external to the cell for pre-baked systems.

The casting and cooling of ingots are the final operations at a reduction plant. Some integrated facilities allow for transportation of molten aluminum directly to rolling facilities. The steps described above are shown in Figure C.12.

Heat Transfer Operations

As was noted above in the process description, the electrolytic reduction cell is the dominate heat transfer operation in a primary aluminum plant. Other operations that will also be discussed in this section are anode baking and casting. 


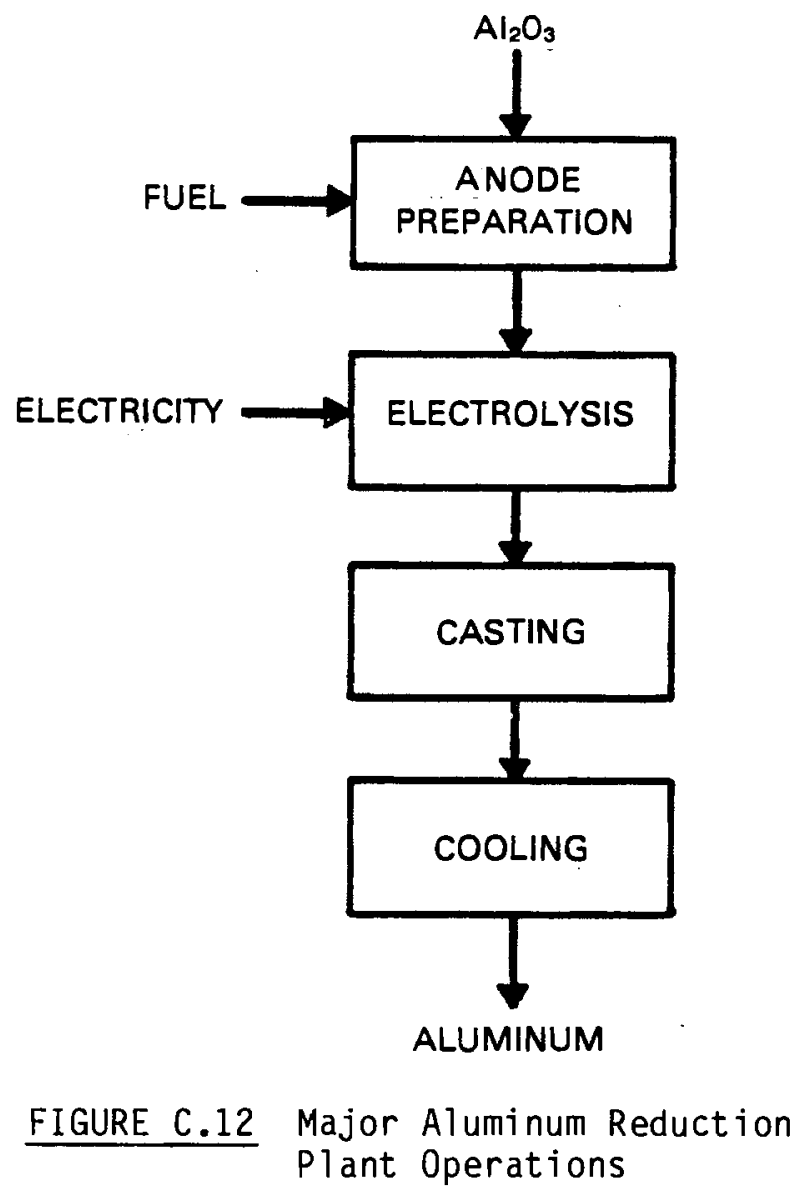

\section{Electrolytic Reduction}

The electroytic reduction cells are the center of activity at an aluminum reduction plant. A diagram of a single cell is shown in Figure C.13. A single cell consists of a refractory lined steel pot, with an additional carbon liner that serves as the cathode. A carbon anode is suspended over the center of the pot and hangs down into the electrolytic bath. Alumina is periodically charged to the continuously operated pot and is dissolved in a molten bath of cryolite, fluorspar and aluminum fluoride. Aluminum is reduced via a series of complex reductions and collects at the bottom of the pot on the cathode. The oxygen that is liberated from the alumina reacts with the carbon anode to form $\mathrm{CO}_{2}$ and $\mathrm{CO}$. The mixture of $\mathrm{CO}$ and $\mathrm{CO}_{2}$ exits the cell with several volatile 


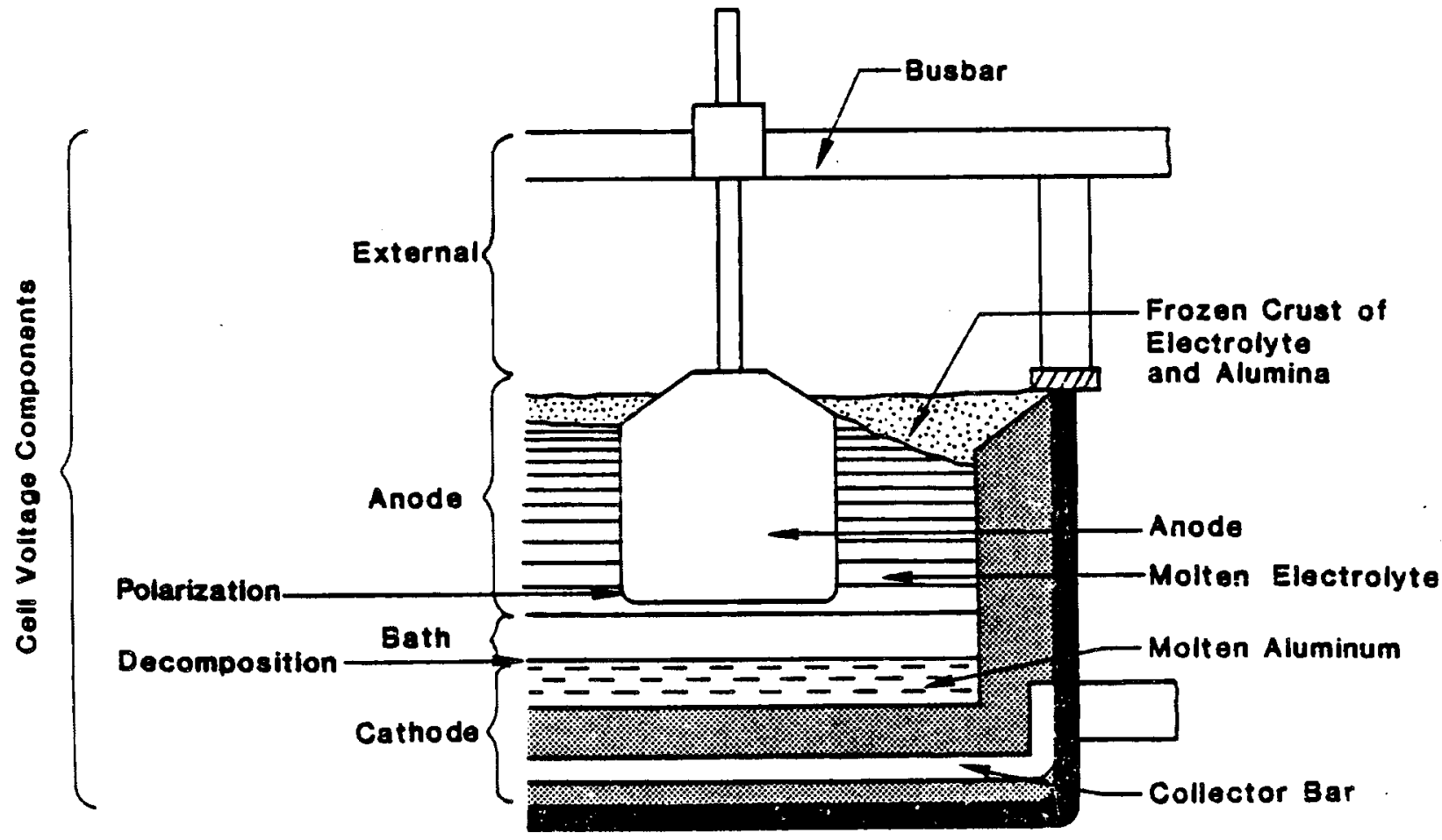

FIGURE C.13 Aluminum Reduction Cell (Shen 1980)

fluoride compounds. The electric current provides energy to drive the reduction reaction and to keep the pot mixture molten at a temperature of about $1700^{\circ} \mathrm{F}$. Additional thermal energy is supplied by the oxidation of carbon anodes. Thermal losses from the cells are inhibited by a crust of alumina and cryolite, which forms on the surface of the bath.

Electrical energy consumption in the reduction cell varies from 14,000 to $16,000 \mathrm{kWh}$ per ton of aluminum, depending on the efficiency of the cell. The theoretical minimum to drive the reaction is $7380 \mathrm{kWh} / \mathrm{ton}$. In addition to the electrical input, approximately $14 \times 10^{6} \mathrm{Btu} / \mathrm{ton}$ are added by the oxidation of the anodes. An individual cell has a voltage drop of 4 to 5 volts and operates at a current of about 100,000 amps. Long rows of cells, called potlines, are operated in series. A single potline may consist of 50 to 180 cells (Shen 1980). 


\section{Anode Baking and Casting}

The carbon anodes consumed in the reduction cells are manufactured from petroleum coke and pitch. Approximately half a ton of coke and 300 lbs of pitch are consumed per ton of aluminum product. Calcined coke and pitch are crushed, mixed, and molded into anode shapes and then baked in a pit-type ring furnace or a tunnel kiln (Alcoa 1983; Kaiser 1983). Petroleum coke is preferred to coal-based coke because of its lower ash content. The baking operation occurs at temperatures between 2000 and $2400^{\circ} \mathrm{F}$ (Palmer et al. 1980). Energy estimates for anode baking vary between 2.3 and $3.6 \times 10^{6} \mathrm{Btu} / \mathrm{ton}$ of aluminum; the median value is nearly $2.6 \times 10^{6}$ Btu/ton (Bodine and Vitullo 1980).

Casting the molten aluminum is a relatively simple operation, but consumes a significant amount of energy. Bodine and Vitullo (1980) reports energy consumption ranging from 1.5 to 11 Btu/ton, depending on the type of casting. The energy required for the casting of sows was reported as being $1.5 \times 10^{6}$ Btu/ton although the average for this operation was reported to be $5 \times 10^{6}$ Btu/ton (Bodine and Vitullo 1980). Latour and Menningman (1981) estimates the average for casting a mixture of slabs, billets, and sows to be $4 \times 10^{6}$ Btu/ton.

\section{Waste Heat Streams}

The waste heat recovery potential in an aluminum reduction plant is large in theory, but the practical applications seem to be limited. The off-gases (mostly $\mathrm{CO}$ and $\mathrm{CO}_{2}$ ) from the potlines have a temperature of about $1800^{\circ} \mathrm{F}$ and carry away between $6 \times 10^{6}$ and $13 \times 10^{6}$ Btu/ton of aluminum produced according to Reding et al. (1975) and Hamell et al. (1980). However, employees of Alcoa and Kaiser indicated that the off-gases from the reduction cells were typically not recuperated because of relatively low energy density, difficulty in collection, and more concern with pollution control and recovery of valuable fluoride compounds. The energy content in anode baking and ingot melting furnace off-gases (estimated by Reding et al. (1975) and Hamell et al. (1980) to be a combined $5.4 \times 10^{6}$ Btu/ton) were similarly discounted. The energy requirements of the reduction cell are so dominating that industry believes 
their time is better spent exploring ways to improve cell efficiency, such as lowering the electrical resistance of anodes, cathodes, and the electrolyte.

In addition to the above information sources, Latour and Menningman (1981) have surveyed the waste heat discharges of the aluminum industry. They found that a total of $53.2 \times 10^{12}$ Btu were rejected in the form of flue gas and $5.2 \times 10^{12}$ Btu were rejected via wastewaters on an annual basis. Their results were summarized in Table C.11.

TABLE C.11 Quantity and Quality of Waste Heat Discharged in the Primary Aluminum Industry (Latour and Menningman (1981)

Temperature Range $\left({ }^{O} \mathrm{~F}\right) \quad 10^{12} \mathrm{Btu}$

125 2

$125-500$ 47

$500-1000$ 3

$1000-1500$

1500 
APPENDIX D

PAPER AND ALLIED PRODUCTS - SIC 26 


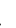

. 
APPENDIX D

PAPER AND ALLIED PRODUCTS - SIC 26

The paper and allied products industry includes the production of pulp, paper and paperboard; conversion of paper and paperboard into end products, and the manufacture of building paper and board.

Paper and paperboard are essential commodities. Annual U.S. per capita consumption is over 600 lbs (Hersh 1981). The industry is highly capital intensive and among the largest consumers of purchased fuels and electricity and of fresh water. According to the 1980 Annual Survey of Manufactures, (U.S. Department of Commerce 1982) this industry ranks third at the 2-digit SIC level in energy use and accounts for $10 \%$ of industrial energy use. Table D.1 ranks the major processes within this classification according to purchased energy use.

Three milling operations pulp, paper and paperboard, consume $88.7 \%$ of the industry's purchased fuels and electricity. Together they reject over 0.3 quads $\left(3.25 \times 10^{14}\right.$ Btu) of waste heat each year (Latour 1981). Figure D.1 presents an energy flow diagram for a modern integrated mill.

Table 0.2 shows what percentages of the heat rejected by this industry can be expected at various temperatures.

TABLE D.1 Ranking by Purchased Energy Use - Paper and Allied Products (U.S. Department of Commerce 1982)

\begin{tabular}{llc} 
SIC & \multicolumn{1}{c}{ Paper Mills } & $\begin{array}{c}\text { Percent of SIC } 26 \\
\text { Energy Use }\end{array}$ \\
2621 & $\begin{array}{l}\text { Paper mills (excluding building } \\
\text { Papers) }\end{array}$ & 45.6 \\
2631 & Paperboard mills & 35.8 \\
2611 & Pulpmills & 7.3 \\
2653 & Corrugaed, solid fiber boxes & 2.8 \\
2661 & Building paper and board mills & 2.8
\end{tabular}

D. 1 


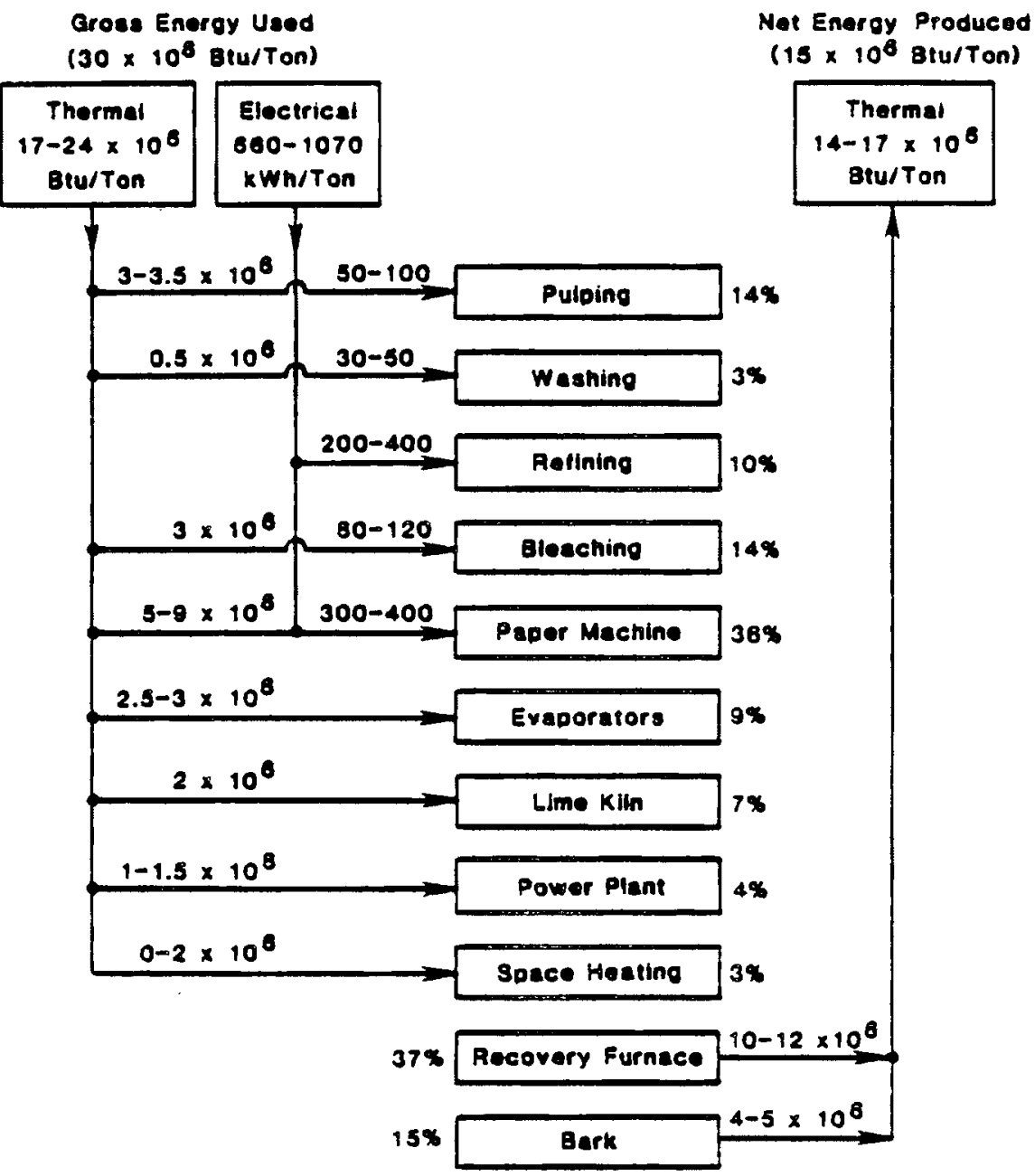

FIGURE D.1 Energy Flows in a Modern Integrated Mill (DOE 1977)

TABLE D.2 Heat Rejection - Paper Industry (SIC 26) (Reding 1975)

\begin{tabular}{lc} 
Temperature of & \% of Total Heat Rejected \\
\cline { 2 - 2 } be low 212 & 56.3 \\
212 to 482 & 30.7 \\
482 to 1472 & 5.0 \\
(radiation, & 8.0 \\
convection, & \\
conduction, & \\
other) &
\end{tabular}

0.2 
Energy purchased by the pulp and paper industry supplied about $52 \%$ of the demand. The remaining $48 \%$ of the industry's energy requirements were generated from renewable process wastes, such as spent pulping liquors and wood wastes (Melion Institute 1983). Table D.3 presents an energy-use pattern for the paper industry.

The following sections discuss manufacturing processes, major heat transfer operations, and waste heat streams.

\section{PULP MILLS}

As defined in SIC 2611, pulp mills are primarily concerned with the production of pulp from wood or alternate fiber sources such as wastepaper, cotton, flax, rags, or straw. They produce market pulp for sale and do not manufacture paper, paperboard or converted products.

TABLE D.3 Energy-Use Pattern - Paper Industry

(API Statistics, Source: Mellon Institute 1983)

\begin{tabular}{|c|c|}
\hline Purchased Fuels & $1980(\%)$ \\
\hline Electricity & 6.2 \\
\hline Coal & 10.5 \\
\hline Residual oil & 16.1 \\
\hline Distillate oil & 0.4 \\
\hline Natural gas & 17.7 \\
\hline Other & 1.0 \\
\hline Total purchased & 51.9 \\
\hline Self-generated & $1980(\%)$ \\
\hline Hogged (wood waste) & 5.4 \\
\hline Bark & 4.7 \\
\hline Spent liquor & 37.2 \\
\hline $\begin{array}{l}\text { Self-generated } \\
\text { hydroelectric }\end{array}$ & 0.4 \\
\hline Other & 0.4 \\
\hline Total self-generated & 48.1 \\
\hline
\end{tabular}

D. 3 


\section{Process Description}

During pulping operations the cellulosic raw materials (of which $80 \%$ is wood) are separated into discrete fibers for further processing into paper and textiles (Hersh 1981).

The following are units operations commonly encountered during the production of purp.

- Raw material acquisition - This operation involves the harvesting, collection and transportation of the raw material from sawmills, lumbering operations, and/or recycling centers.

- Wood preparation - This step involves conversion of the raw material into a form suitable for pulping. Activities may include debarking and chipping of whole trees.

- Pulping - Wood chips or other suitable raw materials undergo conversion into fibers by chemical and/or mechanical means.

- Stock preparation - Pulp may be washed, screened, thickened and bleached as required.

- Chemical recovery - Recovery practices are usually associated with mills producing Kraft pulp.

From a waste heat recovery viewpoint, operations of interest at a pulp mill occur during the pulping, bleaching, and recovery steps.

Several methods are used for pulping wood. In some, the wood is chipped and then cooked with chemicals under controlled conditions of temperature, pressure, time, and pulping liquor composition. Different chemicals, or combinations of them are used by the various processes. In other methods, wood is reduced to a fibrous state by mechanical means or a combination of chemical, thermal and mechanical action. There are four major pulping processes, three of which are chemical pulps (Kraft, semichemical and sulfite) and one mechanical pulping process (groundwood) that account for over $90 \%$ of all pulp production (CTAS Vol II, 1980). Of these four processes, kraft pulping is the dominant method, accounting for $70 \%$ of the pulp consumed (Hersh 1981). 
Although chemical pulping methods require elevated temperatures to activate chip digestion reactions, it is possible in the Kraft process, and to a lesser extent in the neutral sulfite semichemical (NSSC) process, to recover organic material from the spent pulping liquors that can be burned in a recovery boiler. The steam from the boiler can then be used for process heat or electricity generation. Depleted inorganics from pulping chemicals can be recycled by a chemical recovery system.

In the Kraft (also called sulfate) process, wood chips are heated in batch or continuous digesters at temperatures up to $340^{\circ} \mathrm{F}$ and $150 \mathrm{psi}$ in solutions of sodium hydroxide and sodium sulfide (Authur D. Little 1976). Spent liquors from this process are evaporated in multiple evaporators to produce strong black liquor. The liquor is burned in recovery furnaces for steam and power generation. From the combustion process a smelt is obtained that contains inorganic material. The smelt is treated to obtain (calcium carbonate) lime mud, and the lime mud is calcined in a lime kiln to recover calcium oxide ( $\mathrm{CaO}$. Figure D.2 presents a flow-diagram for Kraft pulping.

Pulp made by the NSSC method requires that the wood chips be processed in a neutral solution of sodium sulfate - sodium bisulfate, and then reduced to a pulp in a disc refiner. Reaction conditions typically are $340^{\circ} \mathrm{F}$ at 100 psi (Hersh 1981).

Chemical recovery in the sodium-based NSSC process is more difficult than in the Kraft process. Many mills evaporate and burn spent liquor without chemical recovery and some practice "cross recovery," which requires sending the liquor to a nearby Kraft recovery system (Vogt 1974). Figure D.3 illustrates a flow diagram for an NSSC pulp mill that evaporates and burns spent pulping liquors to recover energy.

Sulfite pulp is made by cooking the fiber source under pressure in an acidic aqueous solution of calcium, magnesium/or ammonium bisulfite, and sulfur dioxide. Due to effluent problems, sulfite recovery systems are not currently practical (Mellon Institute 1983). 


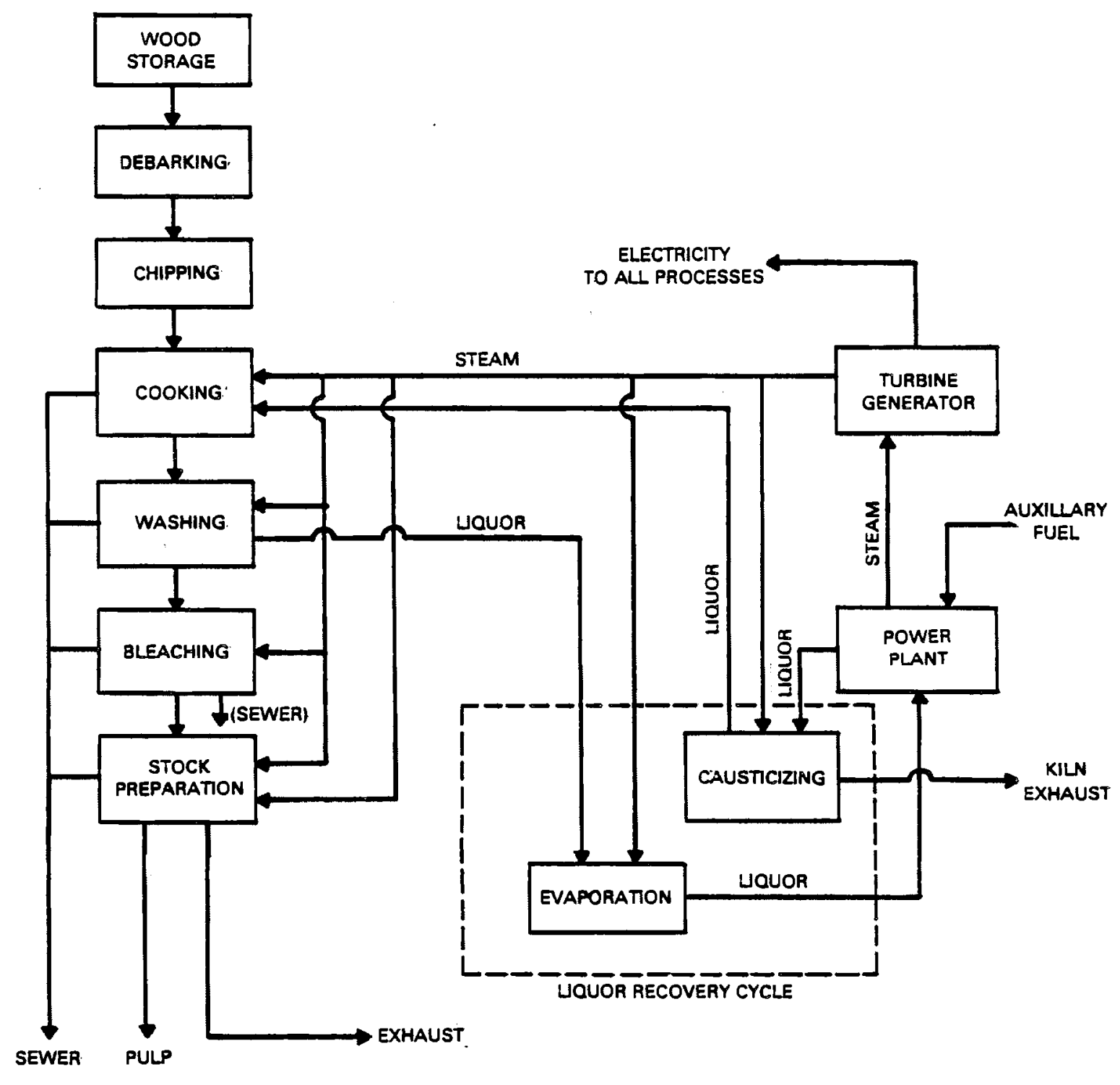

FIGURE D.2. Bleached Kraft Pulping 


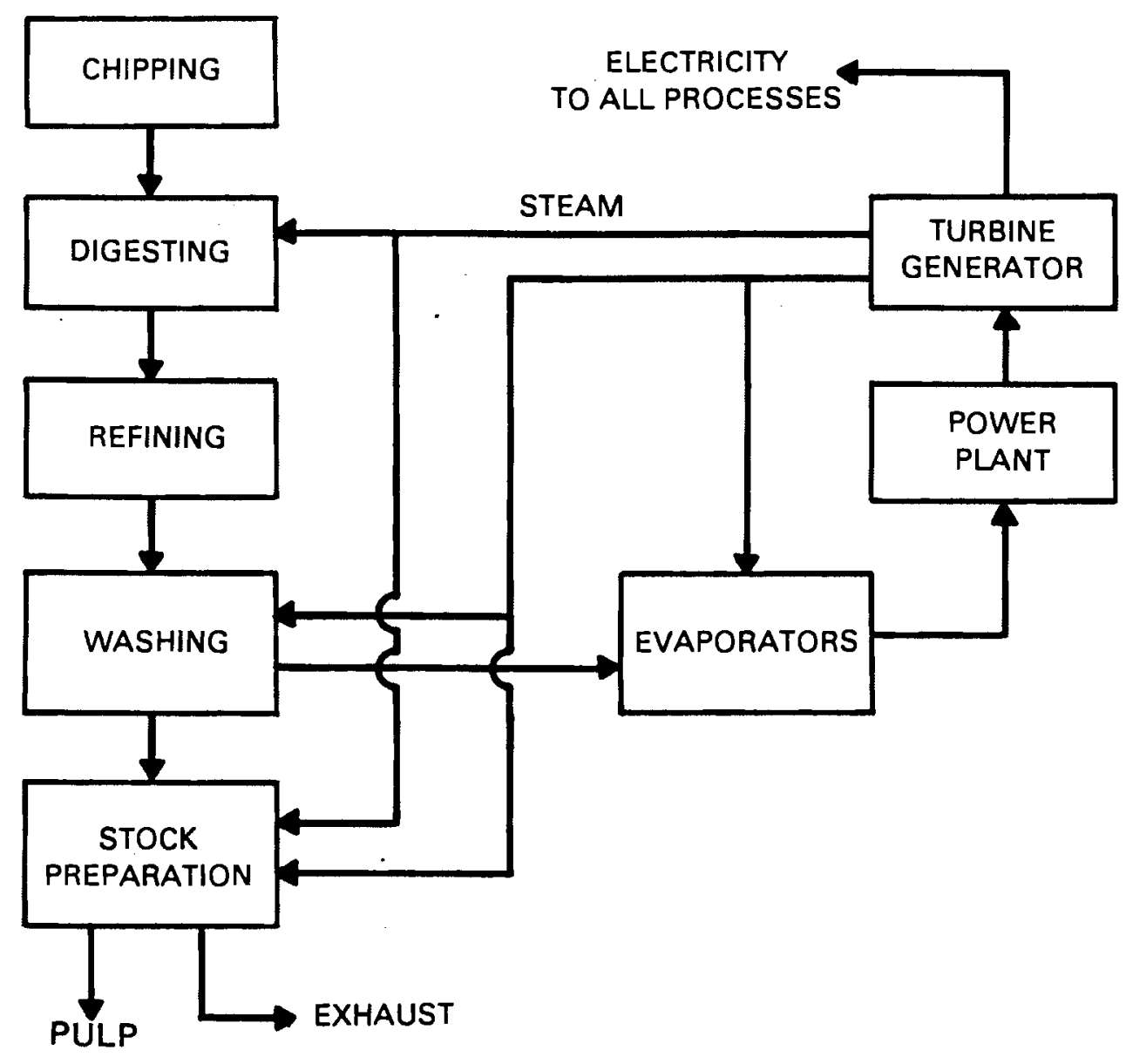

FIGURE D.3 Neutral Sulfite Semichemical Pulping

Groundwood pulp is made by using a water-cooled grindstone to abrade whole logs or wood chips (see Figure D.4). The mechanical action tears apart the wood fibers and an aqueous slurry is obtained. The grindstone is immersed in recirculating water that reaches a steady-state temperature of about $140^{\circ} \mathrm{F}$ (Hersh 1981). The major energy input is electricity to drive the grindstones.

After the fiber source has been pulped, it may require bleaching. Bleaching produces whiter papers by removing or converting the colored matter still present in the pulp. Softwoods generally require more bleaching than hardwoods, because softwoods usually contain more lignin. Standard bleaching agents based on chlorine, chlorine compounds, and peroxide are used, usually in 


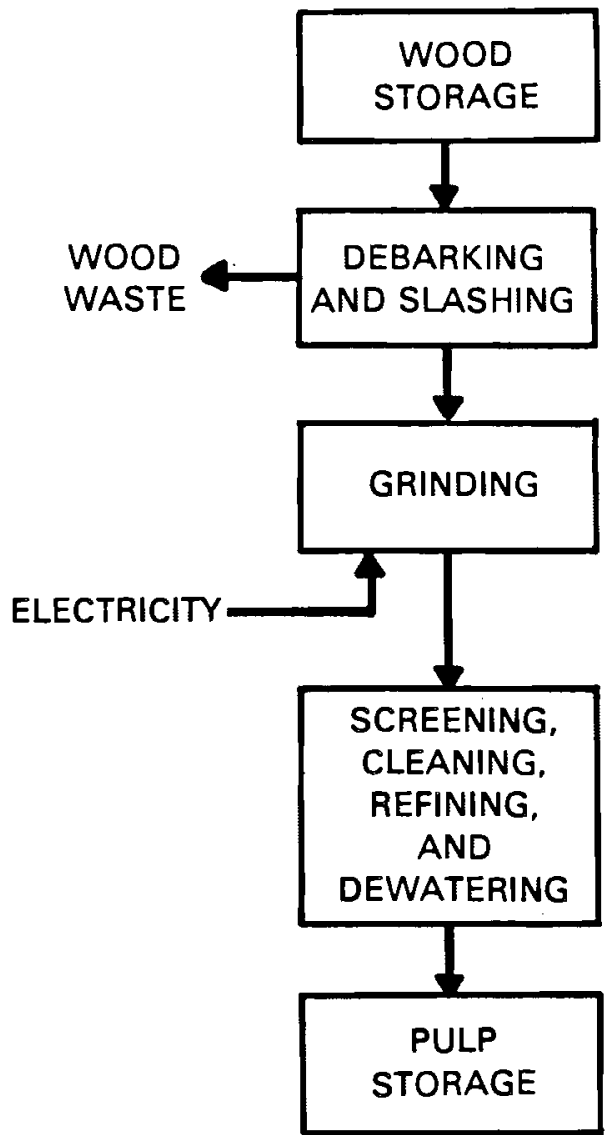

FIGURE D.4 Stone Groundwood Pulping

a variety of sequences designed for the particular pulp being bleached. Energy requirements depend on the type of pulp, fiber source, and degree of brightness desired.

Heat-Transfer Operations

Significant heat-transfer operations occurring at a pulp mill are discussed below.

Digestion

Cooking or digesting of wood chips in the chemical or semimechanical processes can occur in batch or continuous digesters (Figures D.5 and D.6). Table 0.4 shows cooking times and temperatures. 


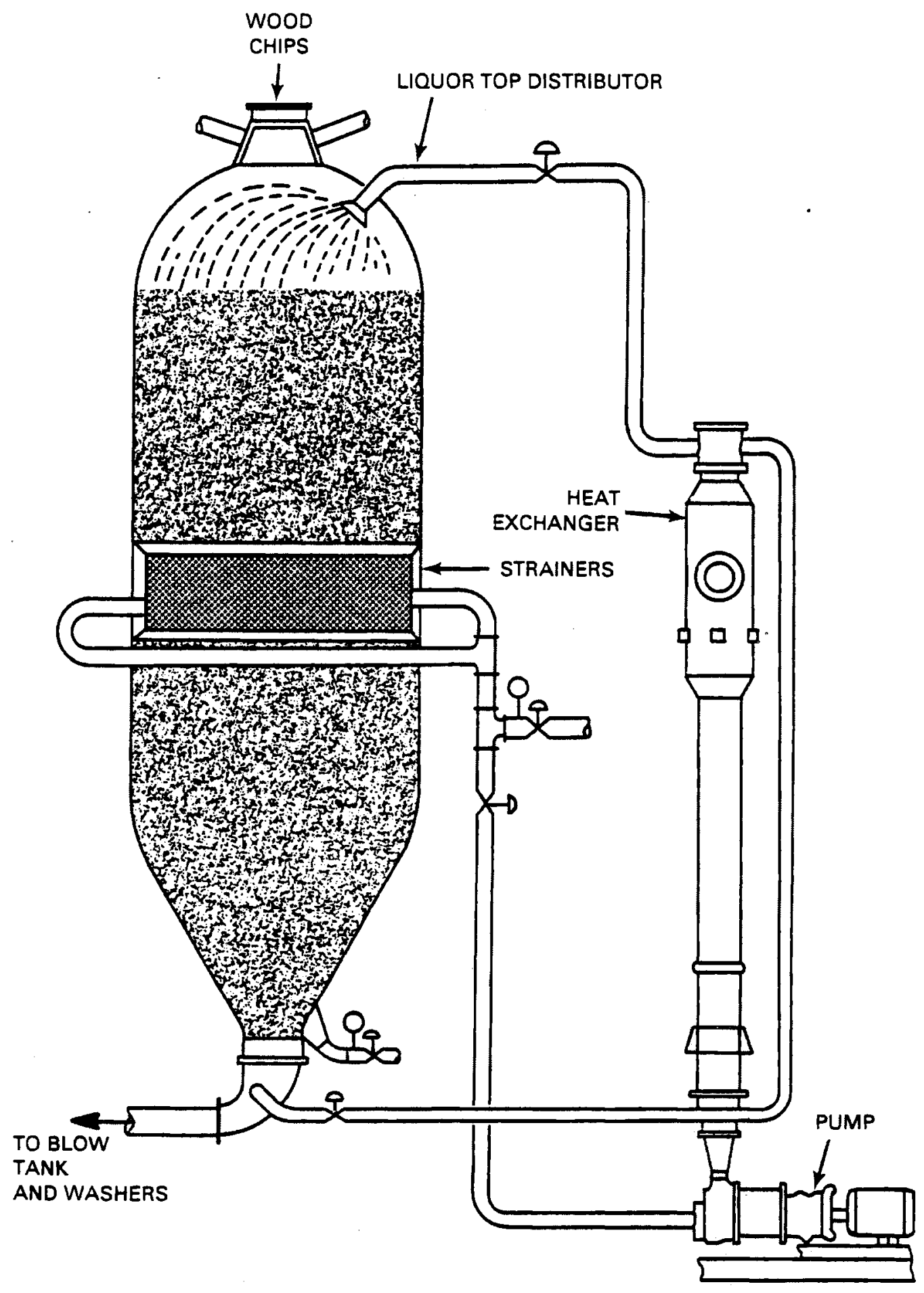

FIGURE D.5. Batch Digestor (Kocureck 1983) 


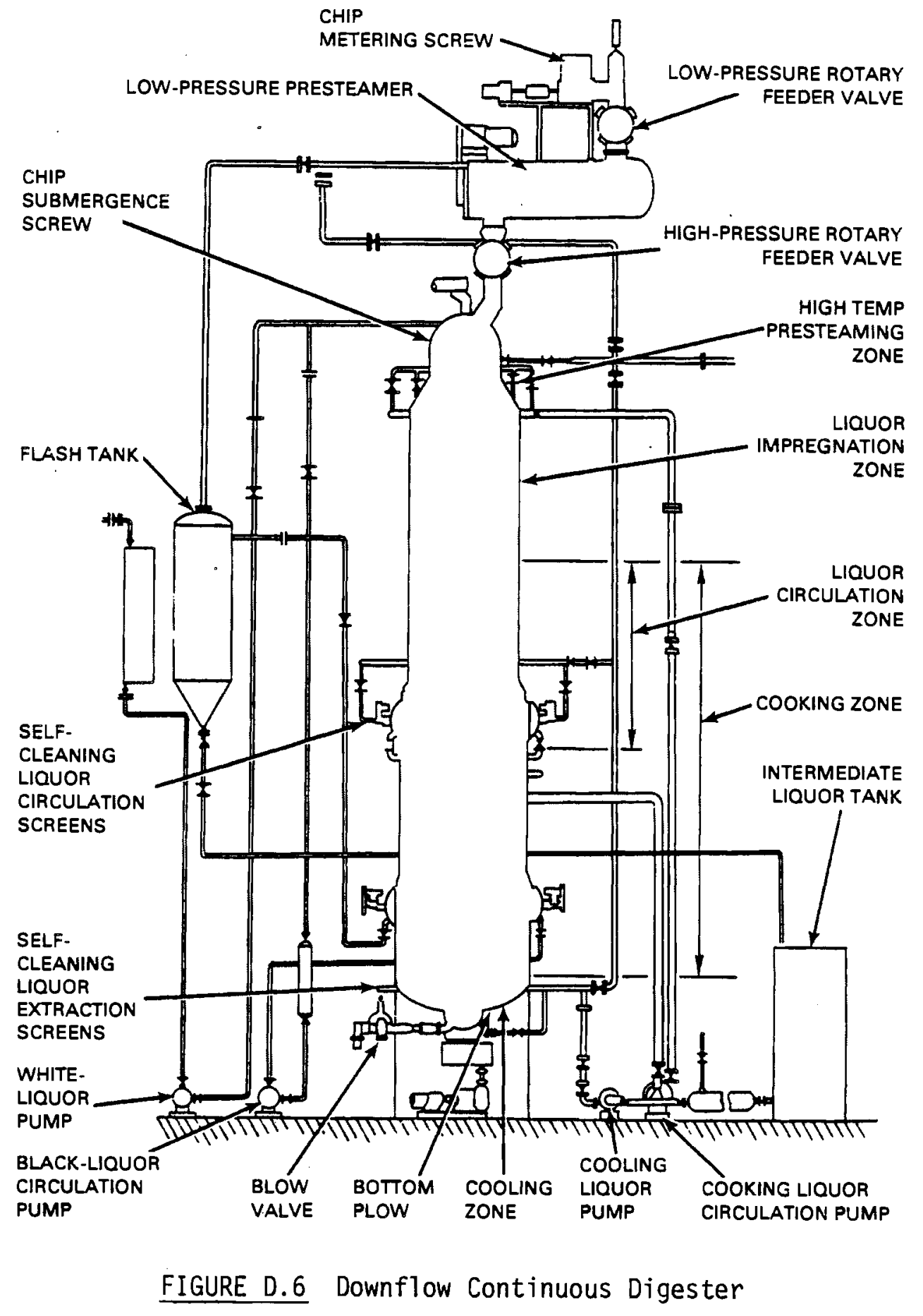

D. 10 
TABLE D.4 Cooking Statistics

(Kozureck 1983)

\begin{tabular}{|c|c|c|}
\hline Method & Time (hris) & $\begin{array}{c}\text { Temperature } \\
\text { Range }{ }_{F} \\
\end{array}$ \\
\hline 1. Kraft & 2 to 5 & 338 to 356 \\
\hline 2. Bisulfite & 3 to 5 & 302 to 320 \\
\hline 3. Acid bisulfite & 4 to 15 & 248 to 284 \\
\hline $\begin{array}{l}\text { 4. Neutral sulfite } \\
\text { semi-chemical }\end{array}$ & $1 / 4$ to $1 / 2$ & 320 to 374 \\
\hline
\end{tabular}

Batch digesters have a reported steam demand of $8,1001 \mathrm{~b} /$ ton pulp and continuous digesters have a demand of 8,700 lbs/ton pulp (Sittig 1977). It is estimated that pulping operations for the kraft process requires $10.67 \times 10^{6}$ Btu/bone-dry ton pulp as an average heat absorption (steam) (Sittig 1977). The different cooking zones, temperatures and cooking time for an example continuous digester are shown in Figure D.7. Cooking statistics will vary from mill to mill depending upon fiber source and pulping method.

Bleaching

Temperatures of bleaching operations can range from 80 to $290^{\circ} \mathrm{F}$ (Sittig 1977). The lower temperatures are representative of mechanical bleaching operations. Electric energy and steam required for pulp bleaching is a factor of the extent of bleaching and nature of the pulp.

Groundwood pulp bleaching uses about $24 \times 10^{3}$ Btu of heat absorbed per bone-dry ton (Sittig 1977). Steam usage in Kraft pulp and bleaching is reported as equivalent to $4.1 \times 10^{3}$ Btu (heat absorded) per bone dry ton pulp (Sittig 1977).

\section{Recovery}

Kraft or NSSC spent cooking liquors are concentrated in multiople-effect evaporators. Table 0.5 shows process conditions for two single-effect evaporators operating at atmospheric pressure. 


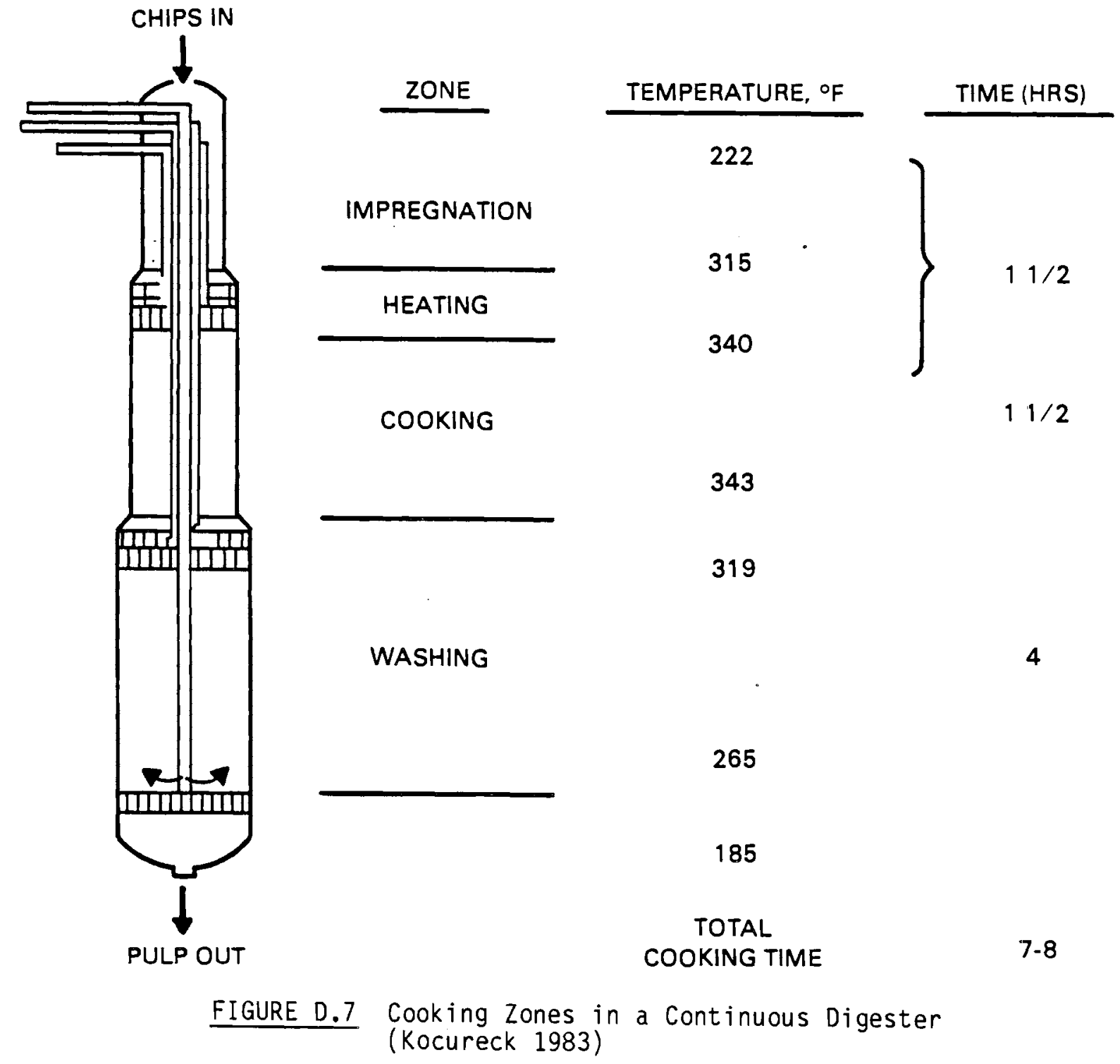

The multiple-effect evaporator (Figure D.8) is usually composed of several units (effects) that are connected in series. Operating temperature in an evaporator is influenced by:

1. temperature of liquor entering an effect

2. latent heat of condensation and vaporization

3. amount of heating surface

4. operating conditions. 


\section{TABLE D.5 Process Conditions for Two Single-Effect Evaporators}

(ERDA 1977)

\begin{tabular}{llll}
\multicolumn{1}{c}{ Process Conditions } & & \multicolumn{1}{c}{$\frac{1}{2}$} \\
\cline { 4 - 4 } Evaporator feed rate, $1 \mathrm{~b} / \mathrm{hr}$ & & 15,000 & 15,000 \\
Evaporator capacity, 1b/hr & & 10,000 & 10,000 \\
Évaporator pressure, psig & & 0 & 0 \\
Steam-chest pressure, psig & & 5 & 12 \\
Steam rates (approx.), 1b/hr & & 12,000 & 12,000 \\
Vapor rate to condenser, $1 \mathrm{~b} / \mathrm{hr}$ & & 10,000 & 10,000
\end{tabular}

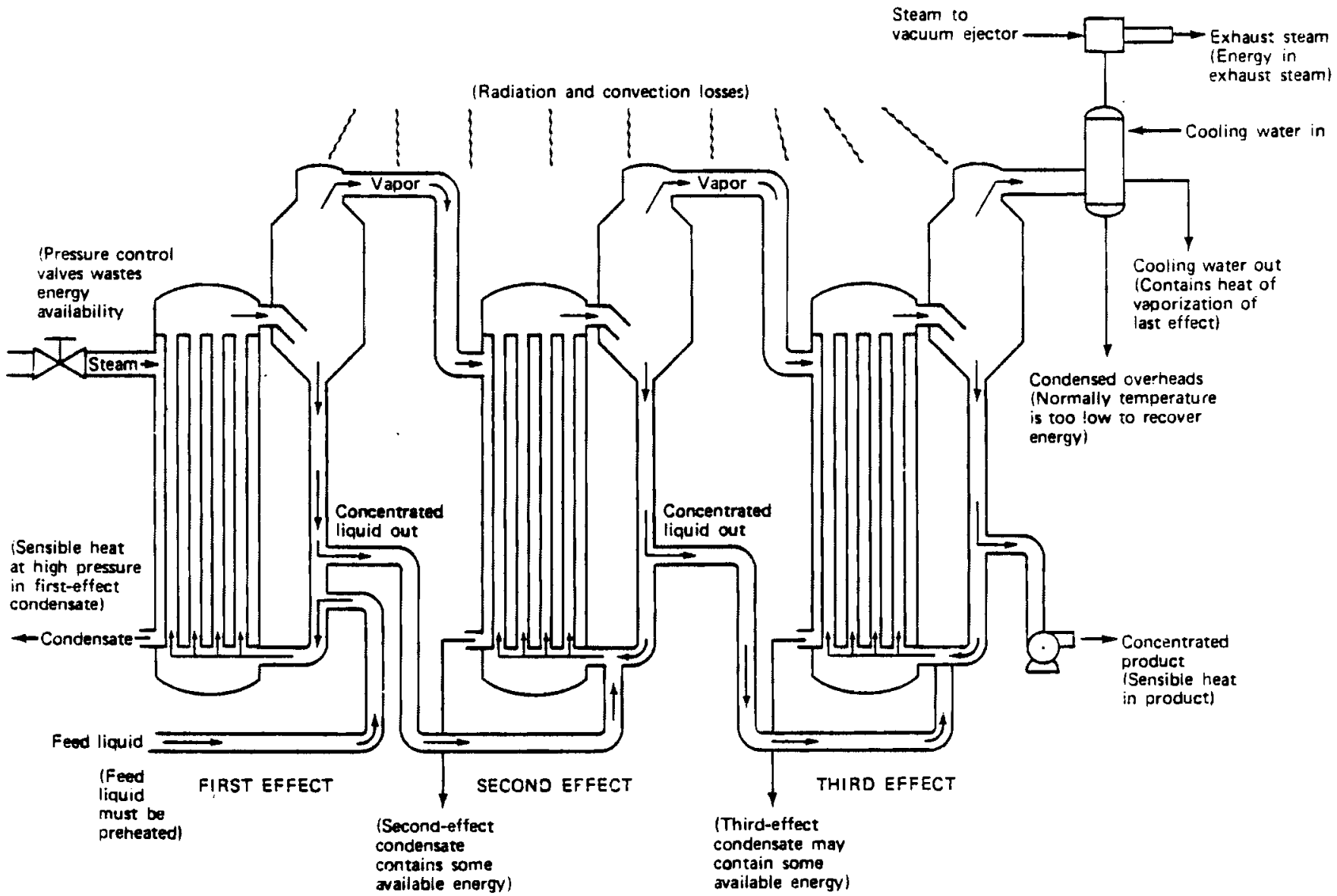

FIGURE D.8 Sources of Energy and Process Flows in the Typical Multieffect Evaporator (ERDA 1977) 
Secondary concentration of the black liquor can occur by direct contact evaporation, which uses hot exhaust gases from the recovery boiler.

When the liquor is concentrated to. $62 \%$ solids it can be burned in a recovery boiler (Jansen 1979). Example operating temperatures are shown in Table D.6. An illustration of a recovery boiler is shown in Figure D.9.

The smelt obtained is dissolved in a dilute basic solution to form "green liquor." Green liquor is treated with calcium oxide ( $\mathrm{CaO}$ ) in causticizers to cause the precipitation of calcium carbonate (also called lime mud). The lime mud is sent to a lime kiln to be converted back to lime at temperatures above $1500^{\circ} \mathrm{F}$ (Battelle 1976). In a simple form, a rotary lime kiln consists of a refractory-lined, open-end steel cylinder inclined about $12^{\circ}$ from feed end to discharge end, and is rotated about the longitudinal axis. Lime kiln sizes range from 6 to 13 feet inside diameter and are 100 to 350 feet long (Mellon Institute 1983). Figure D.10 shows a chemical recovery flow diagram. Waste Heat Recovery

Direct-contact and non-contact heat recovery techniques are practiced by this industry. Table D.7 lists common pulp mill sources of recoverable waste heat.

\section{Digestion}

Waste heat can be recovered from digester blowdown. When the digester is blown during the cooking process, the pressure is reduced and steam is flashed

TABLE D.6 Recovery Boiler Operating Temperatures (Jansen 1979)

\begin{tabular}{lc} 
& Temperature ${ }^{0} F$ \\
\cline { 2 - 2 } 1. Liquor heaters & $220-235$ \\
2. Combustion air & $300-400$ \\
3. Boiler furnace flue gases & $2,200-2,600$ \\
4. Superheater section & $800-1700$ \\
5. Economizer & $350-800$ \\
6. Molten smelt & $1,350-1500$
\end{tabular}




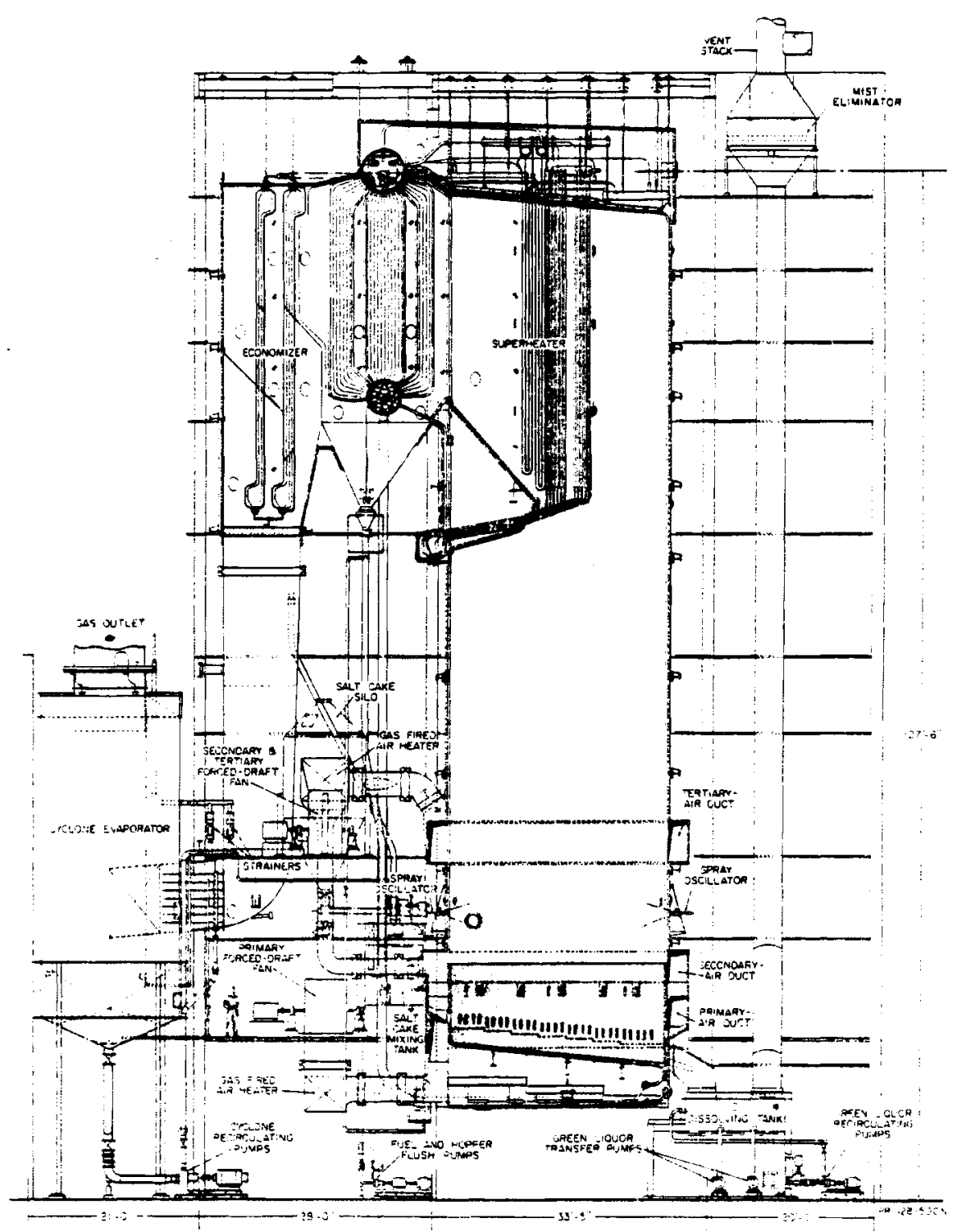

FIGURE D.9 Recovery Boiler (Pierce 1973)

off, resulting in an estimated loss of 2 million Btu/air dry ton of pulp if recovery techniques are not practiced (McDonald 1969).

Two types of recovery systems are commonly used and are classified as 1) the surface condenser, and 2) the jet or direct-contact condenser (McDonald 1969). Table 0.8 presents calculations for an example blow down recovery system. 


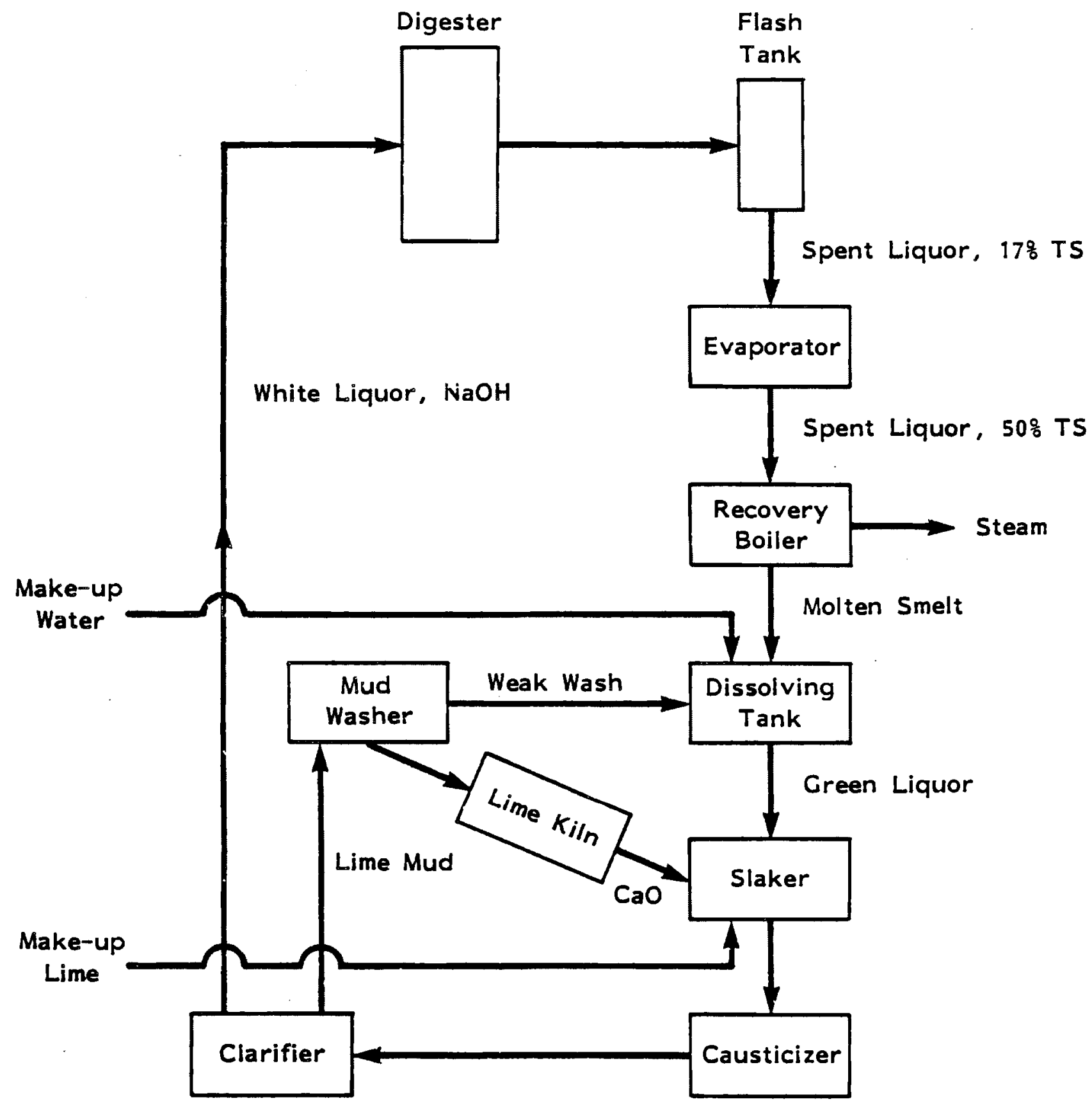

FIGURE D.10. Chemical Recovery Flow Diagram (Battelle 1980) 
TABLE D.7 Major Sources of Recoverable Waste Heat (Gilbreath 1980)

\begin{tabular}{|c|c|c|c|}
\hline Mill Source & Temp ${ }^{{ }^{O}} \mathrm{~F}$ & $\begin{array}{l}\text { orted Ranges } \\
\text { it Available } \\
\text { Btu/ADT }\end{array}$ & Availability \\
\hline 1. Blow heat primary condenser & $195-210$ & $1.5-1.8$ & Intermittent \\
\hline 2. Blow heat secondary cond. & $140-150$ & $0.15-0.25$ & Intermittent \\
\hline 3. Evap. surface condenser & $110-120$ & $2.8-3.2$ & Continuous \\
\hline 4. Turbine condenser & 105 & $0.0-7.5$ & Variable \\
\hline 5. Steam strippers & $130-150$ & $0.4-0.65$ & Continuous \\
\hline
\end{tabular}

TABLE D.8 Example Blowdown Recovery System (McDonald 1969)

Expected

Steam Flash

$2031.31 \mathrm{~b} /$

ton a.d. pulp
Heat rej.

to Sewer

Pulp Prod. (at $110^{\circ} \mathrm{F}$

$78 \mathrm{Btu} /$

1b steam
Average Heat Recovery

$18,200,000 \mathrm{Btu} / \mathrm{hr}$

The quantity of heat that can be recovered will vary from mill to mill and depends on cooking conditions used.

\section{Recovery Operations}

Economizers use waste heat from recovery boiler flue gases to preheat boiler feedwater. Recovery boiler flue gases can also be used by direct contact evaporators to concentrate spent pulping liquors. Basically an economizer consists of enclosed finned heat transfer tubes. The shape and size of a unit depends on the shape of the stack, stream temperature and exhaust flow rate.

Heat recovered through installation of an economizer in the main exhaust stack was reported by one mill as equivalent to $5 \times 10^{16} \mathrm{Btu} / \mathrm{hr}$ (Minnesota Energy Agency 1979).

Hoods can be installed over the hot end of lime kilns to recover heat. These hoods, which can be $100 \mathrm{ft}$ long, contain an air collection pipe the 
length of the hood. One mill uses this system to preheat combustion air and reports heating values of $100^{\circ} \mathrm{F}$ over ambient (Lane 1979).

Another kiln had a heat exchanger added to the exhaust duct system. Combustion air was preheated to $650^{\circ} \mathrm{F}$ and exhaust stack temperatures were reduced from $1250^{\circ} \mathrm{F}$ to $800^{\circ} \mathrm{F}$ (Hogeland 1983).

\section{Waste Streams}

Waste waters and saturated vapors at temperatures less than $350^{\circ} \mathrm{F}$ are the most significant waste heat discharge from pulp mills (Latour 1981). Groundwood pulping has one significant waste heat stream. Waste water from bleaching operations has a waste heat discharge equivalent to $1.10 \times 10^{6}$ Btu/BDT (Latour 1981).

Total useful energy delivered from Kraft pulping operations has been estimated at $42.6 \times 10^{6} \mathrm{Btu} / \mathrm{ton}$ (Streb 1977). Steam flashed during digestor blowdown accounts for approximately $2.8 \times 10^{6}$ Btu/BDT of the pulping operation waste heat (Latour 1981).

In a five stage Kraft pulp bleaching operation, the waste heat content is estimated at $0.4 \times 10^{6}$ Btu/BDT. A three-stage bleaching operation has a discharge of $1.2 \times 10^{6}$ Btu/BDT (Latour 1981). Since most bleaching effluents are sent to the sewer at approximately $100^{\circ} \mathrm{F}$, it is not considered practical to recover the heat (Palmer 1980).

There are two significant rejected heat streams from multiple effect evaporators: 1) saturated steam with an estimated heat content of $3.3 \times 10^{6}$ Btu/BDT, and 2) foul condensate with $1.9 \times 10^{6}$ Btu/BDT waste heat (Latour $1981)$.

The gases that leave recovery boilers at approximately $300^{\circ} \mathrm{F}$ are estimated to have a waste heat discharge of $5.4 \times 10^{6}$ Btu/BDT (Latour 1981).

Off-gases are produced during lime kiln operation. At a temperature of $400^{\circ} \mathrm{F}$ a waste heat discharge of $8.20 \times 10^{5}$ Btu/BDT can be expected (Latour 1981). The lime kiln dryer is estimated to have a waste heat stream of $219 x$ $10^{12} \mathrm{Btu} / \mathrm{yr}$ (Grogan 1981). Exhaust from kiln operations have recoveraple 
heat down to $160^{\circ} \mathrm{F}$ (CTAS 1980). Table 0.9 shows waste heat streams and theoretical energy content.

\section{PAPER AND PAPERBOARD MILLS}

Paper and paperboard mills are establishments primarily engaged in the manufacture of paper and paperboard (paper thicker than 0.012 in.). They may also manufacture converted paper and paperboard products.

Some mills may be integrated, meaning they combine pulping and papermaking at the same site. Because many mills are integrated, energy consumption figures for SIC 2621 and SIC 2631 wi11 reflect energy requirements necessary for pulping operations in addition to the papermaking process. Non-integrated mills buy market pulp to process into paper.

\section{Process Description}

Paper is made by depositing, from a dilute water suspension of pulp, a layer of fiber on a moving wire screen or filament mesh that allows the water to drain and forms a fiber layer. This fiber layer or web is pressed and dried to remove the remaining water. Paper usually leaves the drier section with a 5 to $10 \%$ moisture content (Hersh 1981).

\section{TABLE D.9 Waste Heat Streams - Pulp Mills}

(Latour 1981; CTAS 1980; Palmer 1980)

\begin{tabular}{l} 
Source \\
\hline 1. Ground wood bleaching \\
2. Digestor blowdown \\
3. Kraft bleaching \\
4. Multiple effect \\
evaporators \\
5. Recovery boilers \\
6. Kiln
\end{tabular}

\begin{tabular}{lc} 
Medium & $\begin{array}{c}\text { Reported } \\
\text { Theoretical } \\
\text { Heat Content } \\
\left(10^{\text {Btu/BDT })}\right.\end{array}$ \\
\hline Bleaching effluent & 1.1 \\
Steam & 2.8 \\
Bleach effluent & $0.4-1.2$ \\
Steam & 3.3 \\
Foul condensate & 1.9 \\
Flue gases & 5.4 \\
Off gases & 0.82
\end{tabular}


It is usual for paper to be made on a fourdrinier machine and board or heavy, multilayer papers on cylinder machines. The basic difference between these two machines is in the web formation (Figures D.11 and D.12). A cylinder

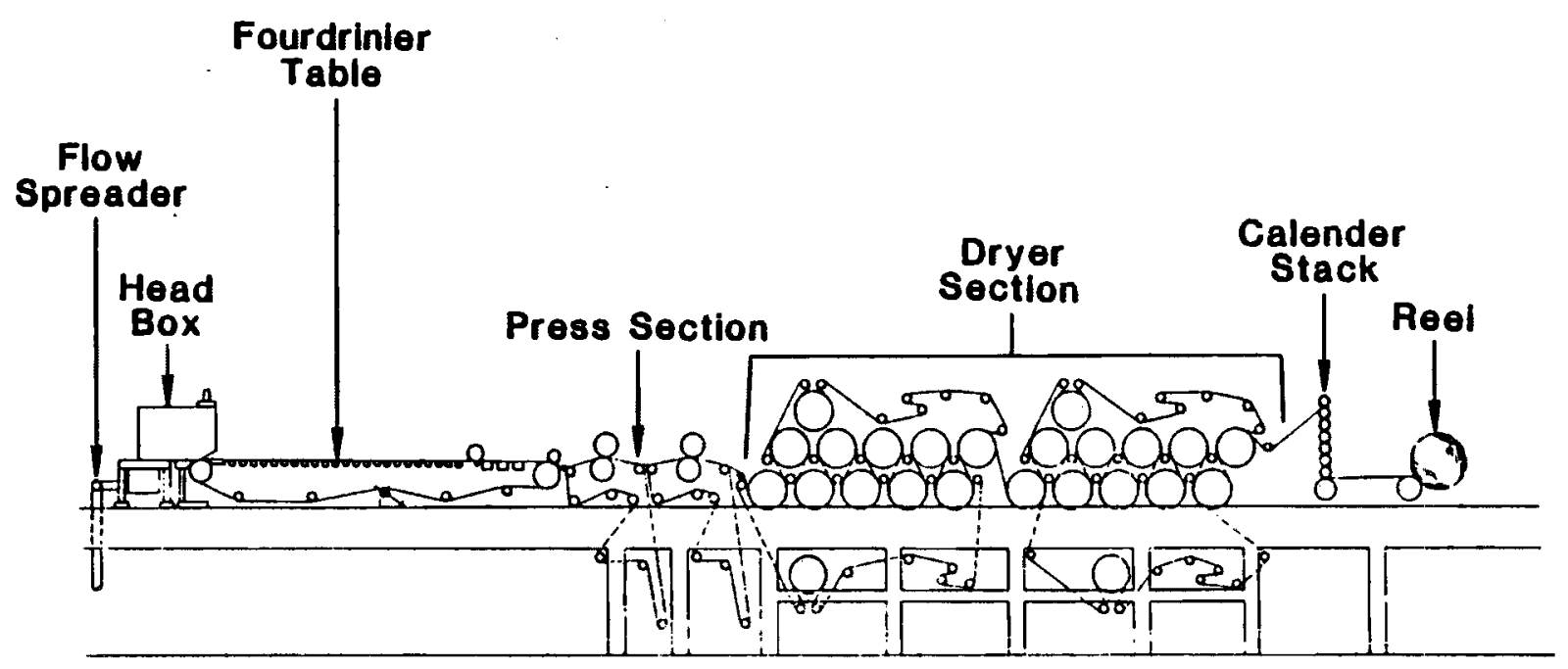

FIGURE D.11 Fourdrinier Paper Machine (TAPPI Press, 1976)

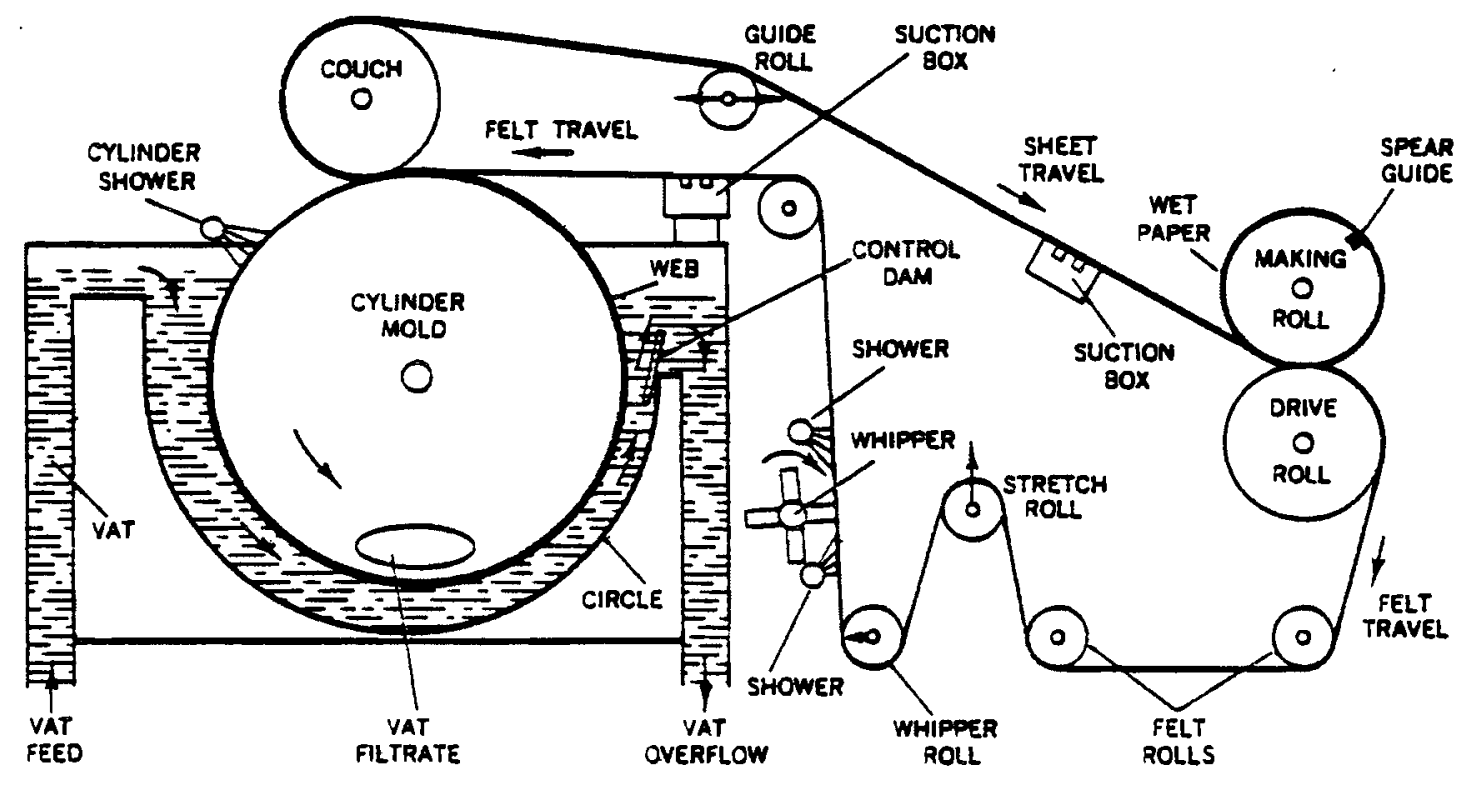

FIGURE D.12 Cylinder Machine Wet End (TAPPI Press, 1976) 
machine has a series of wire-covered cylinder molds that rotate while supporting and draining the moving fiber web. A fourdrinier machine has a flat sheetforming surface. A flow sheet of the fourdrinier operation is presented in Figure D.13.

Heat-Transfer Operations

The major heat transfer operations in the production of paper and paperboard occur during the drying of the paper web.

Drying

Drying is the most energy-intensive unit operation in the production of paper. About two-thirds of the energy used by the forming, pressing and drying section goes into drying the paper (Mellon Institute 1983).

The dryer section is comprised of many rotating heated drying cylinders. The cylinders are usually steam heated and the entire section is often enclosed or hooded. An enclosed hood drying system requires about 10 lbs of air to remove one pound of moisture while open hoods require 25 lbs of air per pound of moisture (Midland-Ross 1983). Air temperatures range from 300 to $800^{\circ} \mathrm{F}$ in the dryer section (McDonald 1960). Electrical energy required in the drier section is usually associated with machine drives.

Waste Heat Recovery

The most common type of heat recovery from the dryer section is the indirect heating of air using cross-flow heat exchange (Midland-Ross 1983).

Using an air-to-air economizer to heat the air, a 50 to $100^{\circ} \mathrm{F}$ temperature rise could be expected in a single pass and from 100 to $300^{\circ} \mathrm{F}$ rise with multiple passes (Midland-Ross 1983). The single-pass would be used for makeup air to the paper machine and multiple passes for makeup to a dryer section.

Heating of water can be accomplished directly or indirectly. The direct heating method requires that water be sprayed directly on the hot exhaust surface or into the hot air stream. This results in dirty hot water that must be filtered. Finned-tube coil heat exchanger systems allow indirect heating of water. 


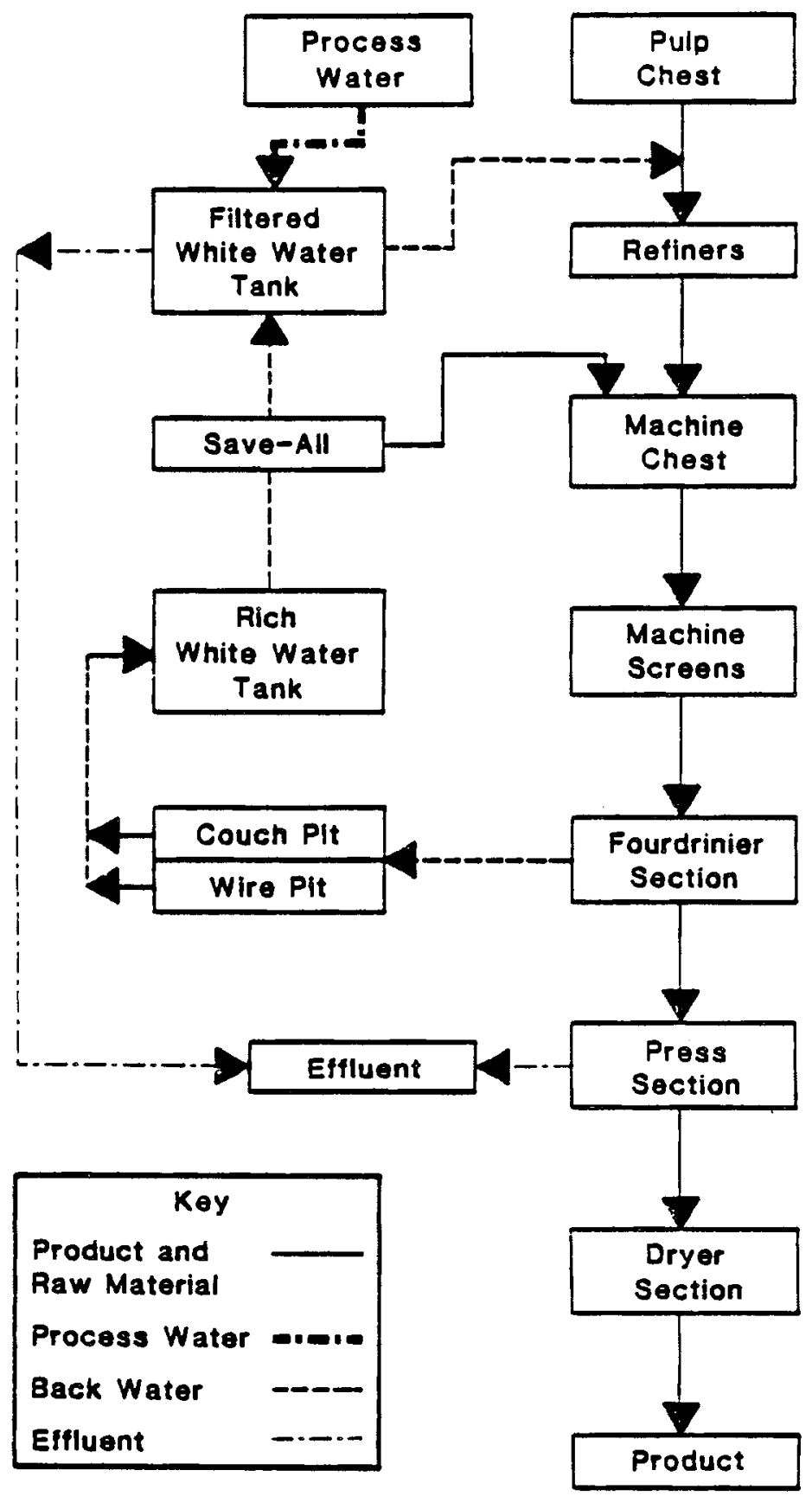

FIGURE D.13 Fourdrinier Paper Machine Process (Hersh 1981) 


\section{Waste Streams}

Waste heat discharged by paper and paperboard mills accounts for about 19 to $40 \%$ of their purchased fuels and electricity. There are two important waste streams: 1) hot moist air with about $3.1 \times 10^{6}$ Btu/BDT of waste heat, and 2) hot water containing about $690,000 \mathrm{Btu} / \mathrm{BDT}$ at a temperature of $250^{\circ} \mathrm{F}$ (Latour 1981). Table D.10 shows different heat contents of exhaust from paper machine hood sections. These are typical values and vary from mill to mill and from wet end to dry end of the hood.

CORRUGATED AND SOLID FIBER BOXES AND BUILDING PAPER AND BOARD MILLS SIC 2653, SIC 2661

Corrugated and solid fiber box plants manufacture products from purchased paperboard, whereas building paper and board mills either purchase market pulp or operate integrated facilities.

In 1979, the construction paper and board production was reported at 5,516 thousand short tons (Post Pulp and Paper Directory 1982). A flowsheet for a typical boxboard operation is shown in Figure D.14.

Unit operations encountered are similar to those discussed in previous sections.

\section{TABLE D.10 Hood Exhaust Heat Contents \\ (Midland-Ross 1983)}

Type of Hood

Open canopy, uninsulated

Insulated totally enclosed

Pulp dryer enclosure

Gas-fired Yankee dryer
Temp. Dry Bulb Dew Point

$\begin{array}{cccc}\left({ }^{\circ} \mathrm{F}\right) & -\left({ }^{0} \mathrm{~F}\right) & & \text { Btu/1b } \\ 130 & 109 & & 111 \\ 165 & 131 & & 170 \\ 275 & 155 & 345 \\ 550 & 164 & & 570\end{array}$




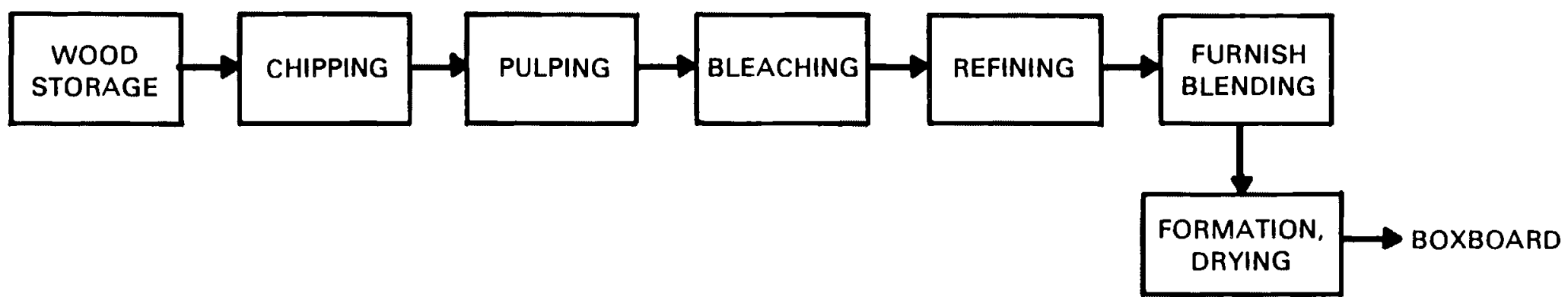

FIGURE D. 14 Folding Boxboard Flow Diagram (Sittig 1977) 
APPENDIX E

STONE, CLAY AND GLASS - SIC 32 


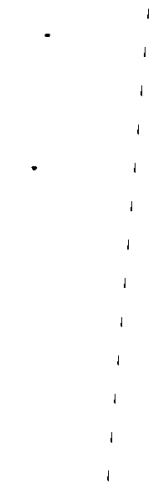


APPENDIX E

STONE, CLAY and GLASS - SIC 32

The major products included in this industry classification are cement, glass (flat, container, pressed and blown), gypsum, brick and structural clay, mineral wool and ready-mix concrete. The production processes for each of these products, with the exception of ready-mix concrete, are characterized by their use of high-temperature, direct-heat operations.

The stone, clay and glass industry ranks fifth in terms of energy use at the two-digit SIC level, accounting for about six percent of the energy used by the manufacturing sector (Radian 1980). Table 5.1 ranks the major processes within this classification according to energy use.

TABLE E.1. Ranking of Stone, Clay and Glass Processes by Energy Use (Bureau of Census 1980)

\begin{tabular}{l} 
SIC \\
\hline 3241 \\
3221 \\
3274 \\
3229 \\
3251 \\
3296 \\
3211 \\
3275
\end{tabular}

Name

Percent of SIC 32 Energy Use

Cement
Glass Containers
Lime
Pressed and Blown Glass
Brick and Structural Clay
Mineral Wool
Flat Glass
Gypsum

34.8

11.8

7.6

5.9

5.1

4.8

4.5

3.8

Three manufacturing processes, cement, lime, and glass (including flat glass, container glass, pressed and blown glass, and mineral wool), consume $70 \%$ of the energy used by this industry grouping. These industries reject almost 0.4 quads $\left(393 \times 10^{12}\right.$ Btu) of waste heat each year in kiln and furnace exhausts (Latour 1981). A description of the manufacturing processes, the major heat transfer operations, and the waste heat streams is provided below for each of the industries.

\section{CEMENT}

At the end of 1980, there were 142 plants producing Portland cement clinker, operated by 44 separate companies (PCA 1980). The plants encompass a wide range of raw materials and product specifications, making it difficult to generalize cement plant design. The following discussion of cement-making processes, heat-transfer operations, and waste-heat streams provides ranges for

E. 1 
the various parameters. These ranges are indicative of the variation in operating parameters encountered in the industry.

\section{Process Description}

Two types of processes are used to manufacture cement: the wet process and the dry process. The basic processing steps (shown in Figure E.1) are the same in both cases, with the exception that in the wet process, raw materials enter the kiln as a slurry rather than as a powder as in the dry process. Currently, about half of the cement produced in the United States is by the wet process (PCA 1980).

Feed preparation involves crushing the limestone rock to mill size, proportioning the feed materials, and, in the dry process, feed drying. The

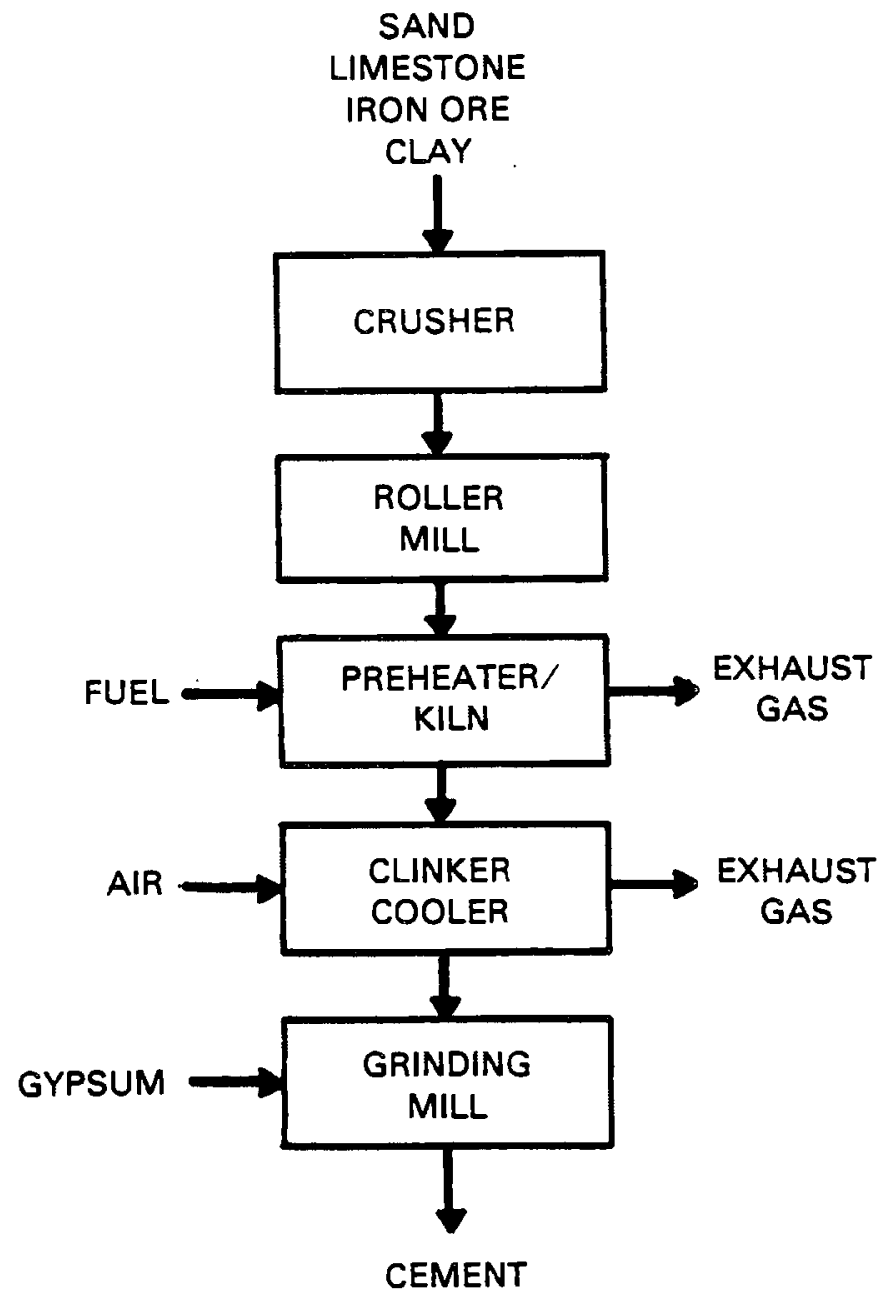

FIGURE E.1. Cement Process

E.2 
grinding step further pulverizes feed materials to a fine powder. In the wet process, water is added during the grinding step to produce a slurry containing about $65 \%$ solids (BuMines 1980).

Calcining or burning involves heating the feed as high as $2900^{\circ} \mathrm{F}$ in a rotary kiln. In the kiln, moisture is driven off, calcium carbonate $\left(\mathrm{CaCO}_{3}\right)$ is converted to calcium oxide $(\mathrm{CaO})$, alkalies are vaporized and any organic material is burned. After calcining, the hot clinker is air-quenched and then fine ground with gypsum to produce cement.

Fossil fuels used to supply direct heat to the kiln account for about $80 \%$ of the energy used in cement manufacture (Sapp 1981). The remaining $20 \%$ is electricity for crushing, grinding, fan power and materials handling. In 1980, $87 \%$ of the cement plants in the United States used coal, 10\% used natural gas, and $1 \%$ used fuel oil (PCA 1980). The trend toward converting to coal is expected to continue, along with an interest in burning waste materials, such as wood chips or waste oils.

\section{Heat-Transfer Operations}

The major heat-transfer operations in the production of cement are burning and clinker cooling. Both are direct-contact operations. Other heat-transfer operations may occur if waste heat recovery is practiced.

\section{Burning}

Burning is the most energy-intensive step in manufacturing cement. Fossi 1 fuels are burned and the hot combustion gases directly contact the feed materials as the two streams move countercurrently through the kiln. Table E.2 shows the events that occur at each temperature zone in the kiln. In the wet

TABLE E.2. Clinker Production-Temperature Zones and Sequential Events Process Temperature $\left({ }^{0} \mathrm{~F}\right)$

$$
\begin{array}{r}
100-300 \\
300-2000 \\
2000-2900
\end{array}
$$
Sequential Events evaporation of water

- vaporize alkalies

- combust organics

- convert $\mathrm{CaCO}_{3}$ to $\mathrm{CaO}$

clinker formation 
process, the water in the slurry is evaporated in the first section of the kiln. The remaining temperature zones are the same in both the wet and the dry process.

Four basic kiln configurations are used to produce clinker: (1) wet kiln, (2) dry kiln, (3) dry kiln with preheater, and (4) dry kiln with precalciner. In each case, the kiln is a rotary kiln which consists of a refractory-lined, cylindrical, steel shell that rotates around an inclined axis. Feed materials enter the top of the kiln and are conveyed by gravity to the lower end where the combustion occurs.

Kiln sizes range from 6 feet in diameter and 120 feet long to 25 feet in diameter and 760 feet long, and rotate at 50 to 90 revolutions per hour (Bumines 1980). In general, the wet process requires the longest kilns since the water must be evaporated. As much as one-fourth the length of the wet kiln contains hanging chains to facilitate heat transfer in the evaporation zone (BuMines 1980). Precalciner and preheater systems allow the use of shorter kilns since much of the preheating and calcining occurs in this equipment before the feed enters the kiln.

Suspension preheaters (see Figure E.2) are used with dry kilns to provide for preheating the incoming kiln feed by direct contact with the kiln exit gases. Between 40 and $50 \%$ of the feed is calcined in the preheater, reducing energy requirements to about $3.0 \times 10^{6}$ Btu/ton of clinker (Garrett 1980). If auxiliary fuel burning is introduced in the last stage of the preheater, up to $90 \%$ of the calcining occurs before the feed enters the kiln (UTC 1980). The latter configuration is referred to as a precalciner.

The theoretical enthalpy requirement for clinker manufacture is $1.5 \mathrm{x}$ $10^{6}$ Btu/short ton (Gartner 1982). Table E.3 summarizes the average kiln energy use for each process.

TABLE E.3. Average Kiln Energy Use by Process (Garrett 1980)

\begin{tabular}{lc}
\multicolumn{1}{c}{ Process } & $\begin{array}{c}\text { Average Fuel Use } \\
\left(10^{6} \text { Btu/Ton Clinker }\right)\end{array}$ \\
\hline Wet & 5.15 \\
Dry & 4.2 \\
Dry with Preheater & 3.0
\end{tabular}




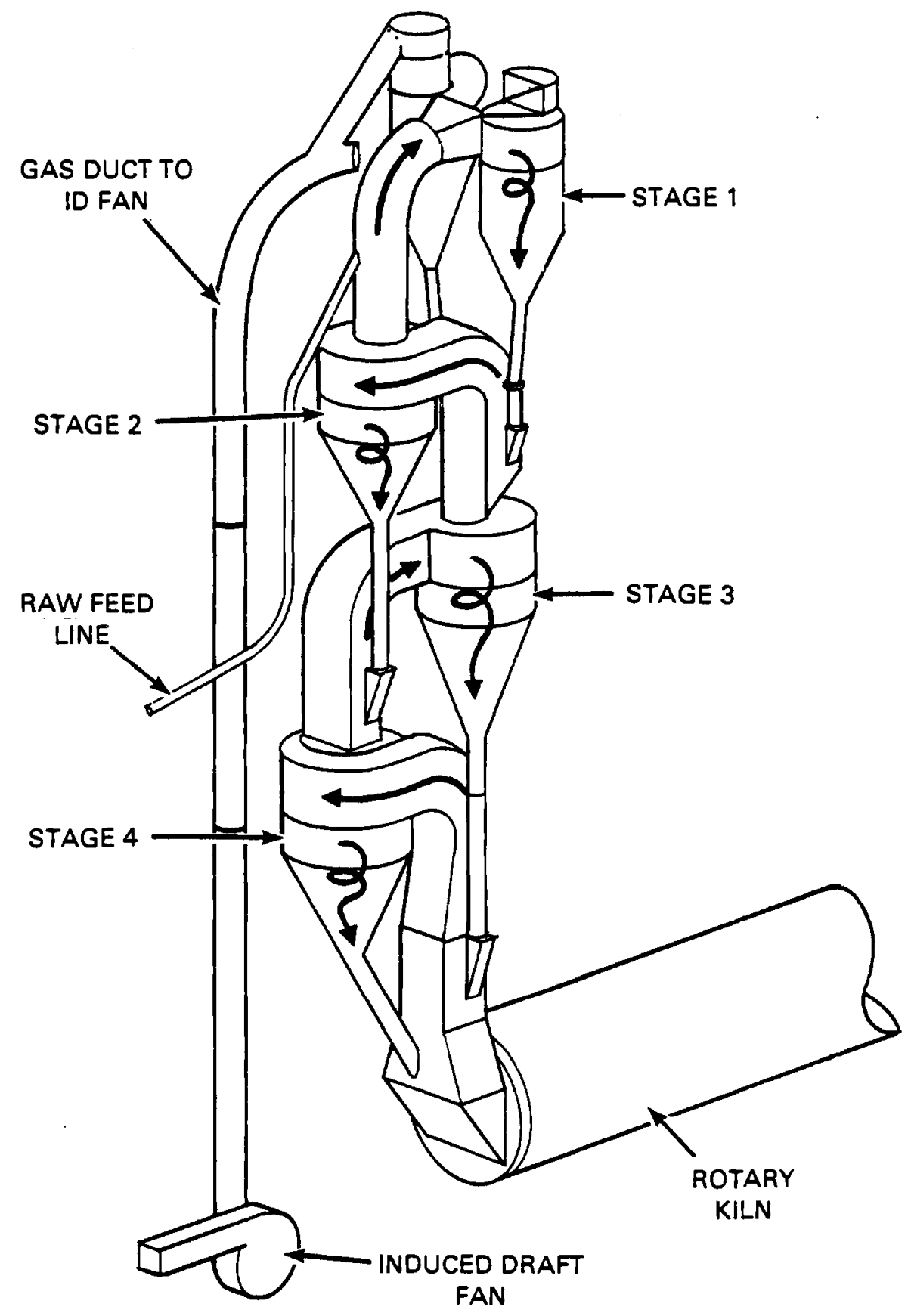

FIGURE E.2 Suspension Preheater 
The fuel efficiency of the precalciner is reportedly 3 to $6 \%$ better than a four-stage preheater (Howlett 1981). Despite their increased fuel efficiency, adoption of preheaters and precalciners in the United States has been slow. Fouling problems have acted as a barrier to their adoption (Marner 1982). Currently about 48 of the long-dry kilns in the United States use suspension preheaters, eleven of which are precalciners (Gartner 1982). Table E.4 summarizes the U.S. clinker capacity and number of kilns by type of process.

TABLE E.4. Number of Kilns and Capacity by Type of Process (PCA 1980)

\begin{tabular}{|c|c|c|}
\hline Process & $\begin{array}{c}\text { Number of } \\
\text { Kilns } \\
\end{array}$ & $\left(10^{6}\right.$ Short Tons/Year $)$ \\
\hline Wet & 175 & 45.6 \\
\hline Dry & 104 & 23.6 \\
\hline Preheater (a) & 48 & 20.2 \\
\hline Total & 327 & 89.4 \\
\hline
\end{tabular}

(a) 11 are precalciners

The average capacity of a single kiln ranges from 170,000 tons/year to $2,322,000$ tons/year with a current average of about 264,000 tons/year (PCA 1980). Table E.5 lists average capacity figures for each type of process. The preheater and precalciner systems have the highest capacity capability and the wet process systems have the lowest.

TABLE E.5. Typical Kiln Capacities (PCA 1980)

\begin{tabular}{lc} 
& \multicolumn{1}{c}{ Tons/Year } \\
\cline { 2 - 2 } Wet Kiln & 250,000 \\
Dry Kiln & 300,000 \\
Preheater or Precalciner & $600,000-1,400,000$
\end{tabular}

E. 6 


\section{Clinker Cooling}

Clinker cooling involves air-quenching the hot clinker as it is discharged from the kiln. In the U.S., most clinker is air cooled in a traveling grate or reciprocating grate cooler in which air is passed up through a moving bed (Bumines 1980). The clinker is cooled from about $2500^{\circ} \mathrm{F}$ to about $150^{\circ} \mathrm{F}$ (Marner 1982). The heated air from the cooler is often routed back to the kiln to serve as combustion air.

\section{Waste Heat Recovery}

Both direct-contact and non-contact heat recovery techniques are used to recover waste heat from cement manufacturing operations, al though waste-heat recovery is not practiced extensively. Table E. 6 summarizes the types of heat recovery currently practiced.

TABLE E.6. Current Waste-Heat-Recovery Practices

\begin{tabular}{|c|c|c|}
\hline Waste Heat Source & $\begin{array}{c}\text { Temperature } \\
\left({ }^{\circ} \mathrm{F}\right)\end{array}$ & Use \\
\hline Clinker Cooler Excess Air & Above 700 & Combustion Air \\
\hline Clinker Cooler Vent Air & Below 700 & $\begin{array}{l}\text { Coal Drying or Raw Feed } \\
\text { Drying }\end{array}$ \\
\hline Kiln Exhaust (dry process) & $700-1800$ & $\begin{array}{l}\text { Preheating Feed or Power } \\
\text { Generation }\end{array}$ \\
\hline
\end{tabular}

The high-temperature air from clinker cooling operations is routed to the $k i l n$ to serve as combustion air. The low-temperature vent air may be used to dry coal or raw feed materials. In some cases, the vent air is passed through an air-to-dir exchanger to cool the exhaust to: (1) $150^{\circ} \mathrm{F}$ for recirculation, or (2) to about $400^{\circ} \mathrm{F}$ before routing to a baghouse (Marner 1982).

About $15 \%$ of the $k i l n s$ in the U.S. utilize kiln exhaust gases to preheat feed materials in suspension preheater systems. This is a direct-contact operation. Currently, only four plants recover kiln exhausts to operate wasteheat boilers for power production. These are listed in Table E.7 (Marner 1982). 
TABLE E.7. Waste-Heat Boiler Installations

$\begin{array}{ll}\text { National Gypsum Co. } & \text { Type of Fuel } \\ \text { Alpena, Michigan } & \text { Coal } \\ \text { Gifford-Hill Co. } & \\ \text { Oro Grande, California } & \text { Coal } \\ \begin{array}{l}\text { Medusa Cement Company } \\ \text { Sylvania, Ohio }\end{array} & \text { Coal } \\ \text { Lehigh Portland Cement Co. } & \\ \text { Independence, Kansas } & \text { Natural Gas }\end{array}$

Exhaust gases from kilns and preheaters and preheater by-pass gases are generally cooled to less than about $500^{\circ} \mathrm{F}$ in air-to-air exchangers before entering the baghouse (Marner 1982).

Waste Heat Streams

The theoretical enthalpy requirement for clinker production is about $1.5 \times$ $10^{6} \mathrm{Btu} /$ ton (Gartner 1982). In 1980, the average fossil fuel requirement for clinker production was about $5.4 \times 10^{6}$ Btu/ton, suggesting that about 3.9 $x 10^{6}$ Btu/ton of heat is lost during cement manufacture (Gartner 1982). This amounts to about $266 \times 10^{12}$ Btu of lost heat in 1980 from clinker production. Table E.8 provides an estimated heat balance for an average kiln.

TABLE E.8. Kiln Heat Balance

$\begin{array}{lll} & \frac{\text { Btu/Short Ton }}{2} & \frac{\%}{28} \\ \text { Theoretical Heat Requirement } & 1.5 \times 10^{6} & 44 \\ \text { Kiln Exit Gases } & 2.4 \times 10^{6} & 17 \\ \text { Shell Losses } & 0.9 \times 10^{6} & 10 \\ \text { Clinker Cooling Air } & 0.54 \times 10^{6} & 1 \\ \text { Cooling Water } \quad \text { Total } & \frac{0.05 \times 10^{6}}{5.4 \times 10^{6}} & 100\end{array}$


Reducing shell radiative and convective losses may be possible through better insulation practices or improved insulating materials. However, this source of waste heat is barred from recovery due to practical considerations. Thus, only the exhaust gases and the cooling water will be considered further.

Waste streams and waste stream temperatures are summarized in Table E.9. Non-contact waste cooling water is from two sources. One source is equipment cooling, including $k i l n$ bearings, grinding equipment, air compressors and burner pipes. The other source is cement cooling. Cement cooling removes the heat.generated by finish grinding prior to storage or shipment. The average waste heat rejected in this cooling water has been estimated as shown in Table E.10.

TABLE E.9. Cement Industry Waste Streams and Temperatures

\begin{tabular}{|c|c|c|}
\hline Type & Source & Temperature $\left({ }^{0} \mathrm{~F}\right)$ \\
\hline \multirow{2}{*}{$\begin{array}{l}\text { Non-Contact Cooling } \\
\text { Water }\end{array}$} & 1) Equipment Cooling & $5^{(a)}$ \\
\hline & 2) Cement Cooling & $25(a)$ \\
\hline \multirow[t]{3}{*}{ Exhaust Gas } & $\begin{array}{l}\text { 1) Kiln - wet } \\
\text { dry } \\
\text { preheater } \\
\text { preheater bypass }\end{array}$ & $\begin{array}{r}150 \\
700 \\
500-700 \\
1700-2000\end{array}$ \\
\hline & 2) $\begin{aligned} \text { Clinker Cooler }- & \text { wet } \\
& \text { dry } \\
& \text { preheater }\end{aligned}$ & $\begin{array}{l}150-250 \\
300-450 \\
300-450\end{array}$ \\
\hline & 3) Finishing Mill & 160 \\
\hline
\end{tabular}

a) temperature above ambient

TABLE E.10. Heat Rejected in Non-Contact Cooling water (Latour 1981)

$\begin{array}{cc}\begin{array}{c}\text { Volume } \\ \text { (gal/ton cement) }\end{array} & \begin{array}{c}\text { Waste Heat } \\ \text { (Btu/ton cement) }\end{array} \\ 400 & 16,690 \\ 200 & 41,725\end{array}$


The temperature of kiln exhaust gases varies depending on whether the process is wet or dry, and, if dry, whether a preheater is used. The exhaust gases from efficient, well maintained wet process kilns are too low in temperature and too high in moisture content for practical heat recovery using currently available techniques (Garrett 1974).

Both the quantity and temperature of the exhausts from long dry kilns is greater than those of preheater kilns. However, several drawbacks have limited the use of waste heat fom the long dry kilns (Garrett 1974):

- exhaust gases are above the inlet temperature limits for roller mills

- the high volumetric flowrate would require large-diameter shells for rotary dryers to keep dryer gas velocity low to prevent excessive dust entrainment.

Table E.11 provides estimates of the volume of kiln exit gases for the three types of systems from two sources.

TABLE E.11. Voliume of Kiln Exit Gas per Ton of Clinker

$\begin{array}{lrr}\text { Type of kiln } & \begin{array}{c}\text { (Garrett) } \\ \mathrm{ft}^{3} / \text { ton }\end{array} & \begin{array}{l}\text { (Marner) } \\ \text { wet kilns }\end{array} \\ \text { dry kilns } & 100,000 & 140,000 \\ \text { preheater kilns } & 50,000 & 64,000 \\ \end{array}$

In the cement industry, the primary heating and cooling operations are direct contact. Therefore, exhaust gases from heating operations contain constituents from the product stream as well as from the fuel. Cooling air that directly contacts the product also carries entrained particles from the product. These waste streams are dusty and highly abrasive. Wet kiln exhausts are estimated to contain between 3 and 18 percent dust and dry kiln exhausts are estimated to contain between 25 and 35 percent dust (Sapp 1981).

Table E.12 provides chemical analyses of various kiln dusts. These constituents are responsible for buildups in the kilns, preheaters and waste- 
heat boilers as well as on the surfaces of induced-draft fan blades. Tables E.13 and E.14 provide chemical analyses of the buildups that occur in wasteheat boilers.

TABLE E.12. Chemical Analyses of Kiln Dusts (Bhatty 1981)

\begin{tabular}{|c|c|c|c|c|}
\hline \multirow[b]{2}{*}{ Item } & \multicolumn{4}{|c|}{ (we ight \%) } \\
\hline & $A^{(a)}$ & $\mathrm{B}^{(\mathrm{b})}$ & $c^{(c)}$ & $D^{(d)}$ \\
\hline $\mathrm{SiO}_{2}$ & 14.6 & 13.4 & 11.4 & 16.4 \\
\hline $\mathrm{Al}_{2} \mathrm{O}_{3}$ & 4.0 & 2.66 & 3.48 & 2.89 \\
\hline $\mathrm{Fe}_{2} \mathrm{O}_{3}$ & 2.2 & 1.25 & 1.16 & 2.33 \\
\hline $\mathrm{CaO}$ & 43.5 & 37.0 & 43.2 & 56.68 \\
\hline $\mathrm{MgO}$ & 1.23 & 1.3 & 1.35 & 0.85 \\
\hline $\mathrm{SO}_{3}$ & 16.9 & 2.01 & 0.73 & 4.36 \\
\hline $\mathrm{Na}_{2} \mathrm{O}$ & 0.39 & 0.44 & 0.08 & 0.07 \\
\hline $\mathrm{K}_{2} \mathrm{O}$ & 5.57 & 10.31 & 2.22 & 0.82 \\
\hline $\mathrm{P}_{2} \mathrm{O}_{5}$ & 0.07 & 0.19 & 0.05 & 0.17 \\
\hline $\mathrm{TiO}_{2}$ & 0.19 & 0.15 & 0.16 & 0.17 \\
\hline L.0.I. & 10.20 & 24.04 & 35.24 & 17.86 \\
\hline$F^{-}$ & 0.21 & 0.11 & 0.02 & 0.1 \\
\hline $\mathrm{Cl}^{-}$ & 0.45 & 6.24 & 0.52 & 0.03 \\
\hline
\end{tabular}
a) high sulfate
b) high chloride
c) low chloride - low sulfate
d) moderate sulfate 
TABLE E.13. Typical Chemical Analysis of Waste-Heat Boiler Build-Ups (Bhatty 1983)

\begin{tabular}{|c|c|c|}
\hline $\begin{array}{c}\text { Component } \\
\text { (wt.\%) }\end{array}$ & Sample A & Sample B \\
\hline $\mathrm{SiO}_{2}$ & 14.26 & 12.12 \\
\hline $\mathrm{Al}_{2} \mathrm{O}_{3}$ & 4.38 & 4.00 \\
\hline $\mathrm{Fe}_{2} \mathrm{O}_{3}$ & 2.64 & 2.24 \\
\hline $\mathrm{CaO}$ & 44.16 & 32.40 \\
\hline $\mathrm{MgO}$ & 1.31 & 0.95 \\
\hline $\mathrm{SO}_{3}$ & 23.25 & 30.64 \\
\hline $\mathrm{Na}_{2} \mathrm{O}$ & 0.80 & 1.12 \\
\hline $\mathrm{K}_{2} \mathrm{O}$ & 9.44 & 14.20 \\
\hline
\end{tabular}

TABLE E.14. Compounds in Waste-Heat Boiler Buildups(a) (Bhatty 1983)

\begin{tabular}{|c|c|c|c|c|}
\hline \multicolumn{3}{|c|}{ First Batch } & \multicolumn{2}{|c|}{ Second Batch } \\
\hline Calcite & Calcite & $\begin{array}{c}3 \\
\text { Anhydrite }\end{array}$ & $\begin{array}{l}\quad 1 \\
\text { Calcium } \\
\text { langbeinite }\end{array}$ & Anhydrite \\
\hline $\begin{array}{l}\text { Calcium } \\
\text { langbeinite }\end{array}$ & $\begin{array}{l}\text { Calcium } \\
\text { langbeinite }\end{array}$ & $\begin{array}{l}\text { Calcium } \\
\text { langbeinite }\end{array}$ & $\begin{array}{l}\text { Calcium } \\
\text { oxide }\end{array}$ & $\begin{array}{l}\text { Calcium } \\
\text { langbeinite }\end{array}$ \\
\hline Anhydrite & Anhydrite & Calcite & $\begin{array}{l}\text { Dicalcium } \\
\text { silicate }\end{array}$ & Calcite \\
\hline Quartz & $\begin{array}{l}\text { Calcium } \\
\text { oxide }\end{array}$ & Quartz & Aphthitalite & Quartz \\
\hline $\begin{array}{l}\text { Calcium } \\
\text { oxide }\end{array}$ & Quartz & $\begin{array}{l}\text { Calcium } \\
\text { oxide }\end{array}$ & Syngenite & $\begin{array}{l}\text { Calcium } \\
\text { oxide }\end{array}$ \\
\hline
\end{tabular}

(a) Relative amounts in decreasing order of $X$-ray diffraction intensity 
GLASS INDUSTRY - SIC 3211, 3221, 3229 and 3296

Although the end products differ, the major heat-transfer operation (melting) is the same for each of the product categories listed below:

SIC 3211 Flat Glass

SIC 3221 Glass Containers

SIC 3229 Pressed and Blown Glass

SIC 3296 Mineral Wool

Glass container manufacturing accounts for about $70 \%$ of glass production, flat glass $15 \%$, pressed and blown glass $12 \%$, and fibers $3 \%$ (EPRI 1982).

The following discussion applies generally to the glass industry. Where significant differences occur among product categories, they will be highlighted.

\section{Process Description}

Glass-making operations are typically grouped into the five basic operations shown in Figure E.3: (1) batch handling, (2) melting and fining, (3) forming, (4) post forming, and (5) product handling.

Batch handling involves preparing and transferring the feed to the melting furnace. The feed is primarily composed of sand, soda ash, limestone, and feldspar. In addition, the feed may contain significant amounts of cullet, which is scrap glass. Cullet is used as a batch material to reduce the melting energy requirement and to reduce the amount of dust and particulate matter in the exhaust gases. Some forming operations generate as much as $70 \%$ waste glass which must be recycled as cullet (Kirk-0thmer undated). More efficient operations may require purchasing cullet from flat-glass companies or recycling centers.

Melting and fining occurs in refractory-lined furnaces at temperatures up to about $2800^{\circ} \mathrm{F}$. The temperature of the batch is raised as quickly as possible to the melting temperature where various dissolution, volatilization and redox reactions occur. These reactions are as follows (Kirk-0thmer): 

$1020^{\circ} \mathrm{F}$
$\mathrm{Na}_{2} \mathrm{CO}_{3}+\mathrm{SiO}_{2}$
$\mathrm{Na}_{2} \mathrm{SiO}_{3}+\mathrm{CO}^{2}$
$1290^{\circ} \mathrm{F}$
$\mathrm{Na}_{2} \mathrm{SiO}_{3}+\mathrm{SiO}_{2}$
$\mathrm{Na}_{2} \mathrm{Si}_{2} \mathrm{O}_{5}$
$1435^{\circ} \mathrm{F}$
$3 \mathrm{Na}_{2} \mathrm{Si}_{2} \mathrm{O}_{5}+\mathrm{SiO}_{2}$
forms a mixture approach- ing eutectic

Fining involves holding the glass in the furnace to allow entrapped gas bubbles to escape. The glass must be raised to nearly $2730^{\circ} \mathrm{F}$ to eliminate seeds of entrained air (Berg 1982). Current melting practices of raising glass temperatures to about $2500^{\circ} \mathrm{F}$ represents a trade-off between product quality and energy costs (Berg 1982).

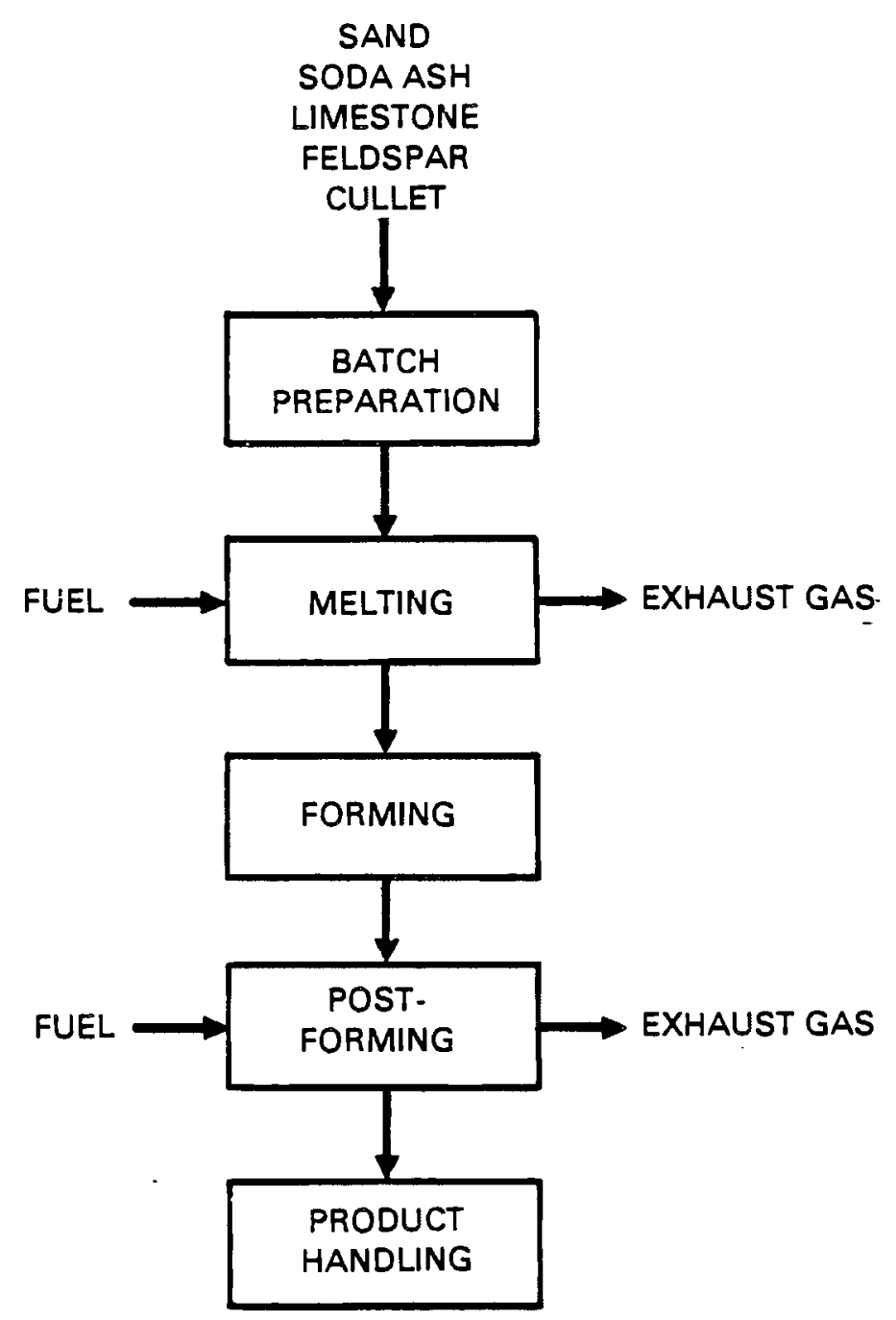

FIGURE E.3. Glass Production Process 
Forming converts the molten glass to an intermediate shape by pressing, blowing, drawing, or casting. A major post-forming operation through which most glass products (except glass fibers) pass is annealing, in which internal stresses are removed. Annealing involves heating the glass to about $1100-$ $1200^{\circ} \mathrm{F}$, followed by controlled cooling.

Natural gas is preferred over other fuels for glass melting because it has been relatively cheap and it is an exceptionally pure fuel. Natural gas accounts for about 80 percent of the energy used by the glass industry (Webb 1982). The remaining melters use fuel oil or are electric. Heat-Transfer Operations

The major heat-transfer operations in the glass industry are melting, heat recovery from melter exhausts, and annealing.

Glass Melting

Melting is the most energy-intensive step in glass manufacture. Typically, the heat transfer occurs through direct contact with hot combustion gases. However, a larger number of glass manufacturers now use resistive electric melting. Current estimates indicate that about $5 \%$ of the energy used for melting is electricity (Berg 1982).

Two basic melter configurations are used to produce glass: (1) pot furnaces and (2) tank furnaces. Pot furnaces are used for melting smaller quantities of glass, particularly special glasses where the melting batch must be protected from the products of combustion. These furnaces are generally inefficient and have poor temperature-control capability.

In tank furnaces, batch materials are charged to one end of the tank and fined glass is pulled from the opposite end in a continuous operation. Small, continuous tanks are used to produce high-quality glasses in low volumes. Tank capacity is about 7 tons/day. Some of the tanks operate as cold-crown units with the unmelted batch covering the molten glass in the premelt zone. Pollution from glass melting is substantially reduced by using cold-crown units. These are electrically heated from the bottom or the sides.

The largest furnaces are continuous regenerative furnaces that recover waste heat from the melter off-gases. These furnaces may be cross-fired or end- 
fired. End-port furnaces fire and exhaust from the back wall forming a Ushaped flame over the glass surface (Kirk-Othmer). Cross-firing allows more even heating across a larger surface. Production capacities range from less than 150 tons/day to over 700 tons/day.

Energy consumption is a function of furnace type and size, and of the type of glass. All flat glass is produced in side-port regenerative furnaces. The capacity and energy use varies considerably, as shown in Table E.15. A number of different types of furnaces are used for container glass. More than half of the container glass is produced in side-port regenerative furnaces. The number of ports on a side varies from 3 to 7 depending on furnace size. Table E.16 shows the types of furnaces used to produce container glass and their energy consumption. Furnaces now used in the container glass industry range in capacity from about 75 to 480 tons/day, with an average capacity of 180 tons/ day (Berg 1982). Because of the wide variety of products produced, a variety of furnaces are used to produce pressed and blown glass. These are listed in Table E.17 along with their capacity ranges and energy use.

TABLE E.15. Flat Glass Furnace Capacity and Energy Use (Schoor 1975)

\begin{tabular}{lcc} 
Capacity & $\begin{array}{c}\text { Energy Use } \\
\left(10^{6} \text { Btu } / \text { Ton }\right)\end{array}$ \\
\cline { 2 - 3 } Sheet & $100-250$ & $12-20$ \\
Plate & $100-300$ & $10-20$ \\
Float & $300-750$ & $6-11$
\end{tabular}

TABLE E.16. Container Glass Furnace Energy Use (Schoor 1975)

Furnace Type

Side-Port Regenerative

End-Port Regenerative

Electric

Electrically Boosted Regenerative

\section{Energy Use}

$\left(10^{6}\right.$ Btu/Ton $)$

$\begin{aligned} 5 & -9 \\ 5 & -14 \\ 3 & -5 \\ 4.5 & -7.5\end{aligned}$

E. 16 
TABLE E.17. Pressed and Blown Glass Furnace Capacities and Energy Use

\begin{tabular}{lcc}
\multicolumn{1}{c}{ Furnace Type } & $\begin{array}{c}\text { Capacity } \\
\text { (Tons/Day) }\end{array}$ & $\begin{array}{c}\text { Energy Use } \\
\left(10^{6} \text { Btu/Ton) }\right.\end{array}$ \\
\cline { 1 - 1 } Side-Port Regenerative & $100-300$ & $5-20$ \\
End-Port Regenerative & $60-150$ & $5-25$ \\
Unit Melters & $<100$ & $12-35$ \\
Electric & & $3-5$ \\
Electrically Boosted Regenerative & -- & $4.5-7.5$ \\
Day Tanks & $1-4$ & $12-50$ \\
Pot Furnaces & 2 & --
\end{tabular}

\section{Waste Heat Recovery}

It is common practice in the glass industry to recover heat from melter exhausts to preheat combustion air. Brick regenerators (checkers) are the predominant heat-recovery device. In the glass industry, approximately $90 \%$ of all glass is melted in regenerative furnaces (A. D. Little 1976). The remaining $10 \%$ is melted in recuperated glass furnaces.

The regenerators are constructed of refractory bricks in an open matrix arrangement (see Figure E.4). Combustion gases are directed across the batch surface and are routed downward through one of the checkers, through the reversing valve and finally to the stack. The hot gases transfer a significant amount of heat to the checker bricks. Combustion air is preheated as it is drawn up through the opposing checker. The reversing valve is alternated at 20 to 30 minute intervals, thus directing the exhaust gas and combustion air to the opposing checkers (Wei 1981).

Checker size averages about $46 \mathrm{ft}^{3}$ per ton of glass melted. Typical gas passage dimensions within the checker range from $4^{\prime \prime} \times 4^{\prime \prime}$ to $6 "$ " $\times 9^{\prime \prime}$ (Webb 1982). Smaller openings have been found to become plugged quickly by constituents in the melter exhaust. 

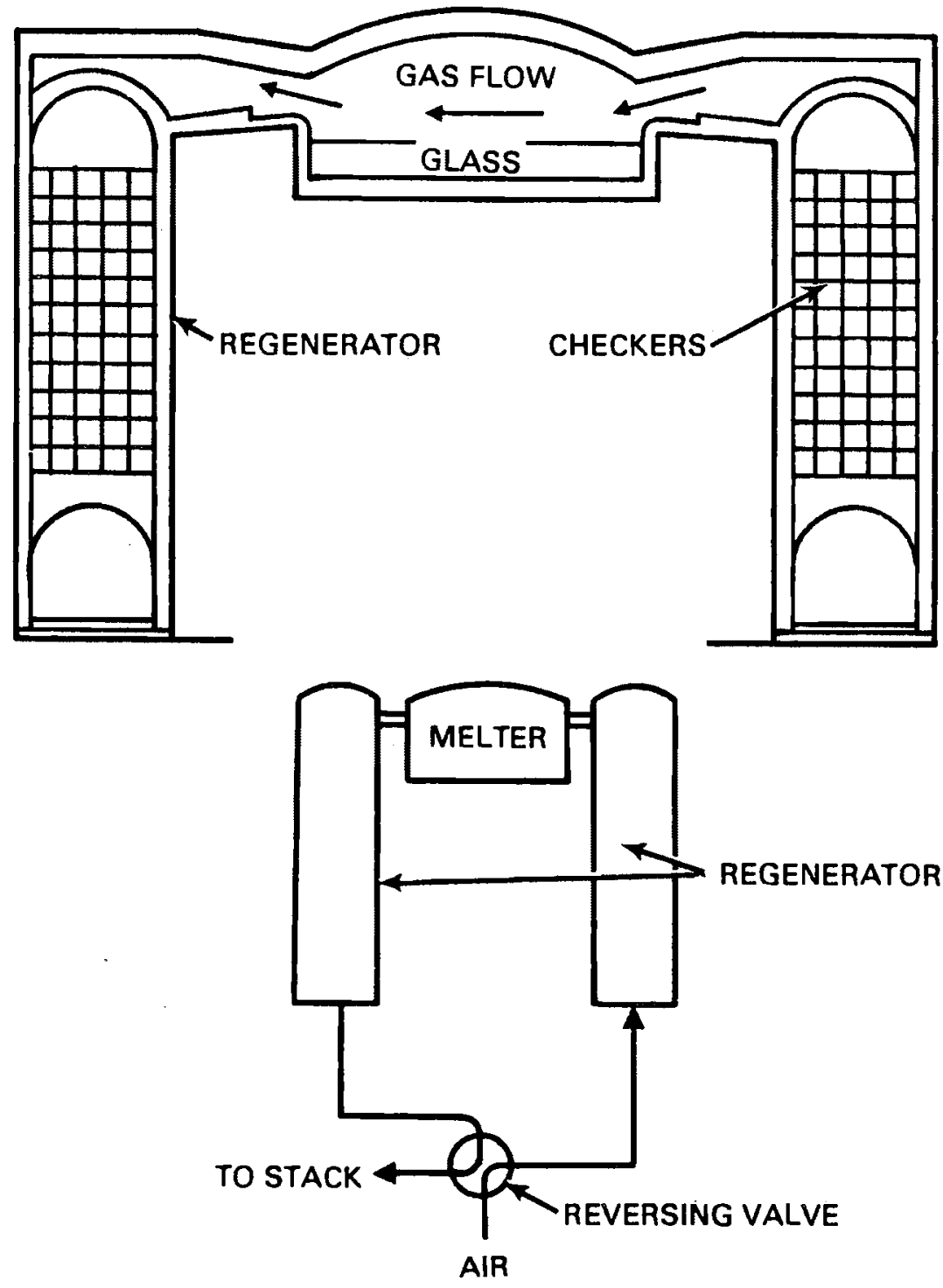

FIGURE E.4. Regenerative Glass Furnace

Chrome-magnesite bricks are typically used in the upper regenerator chamber which must have high-temperature capability to resist slagging and creep due to batch carryover. High-magnesium oxide bricks are used in the upper part of the checker, whereas chrome-magnesite bricks are used in the middle and lower levels of the checkers, because magnesium oxide reacts with $\mathrm{SO}_{3}$ to form magnesium sulfate at temperatures below $2055^{\circ} \mathrm{F}$ (Wei 1981). Direct-bonded chrome-magnesite or super duty fireclay bricks are used for the rider arches which must bear the load of the entire checker. Lower walls are typically constructed of fireclay bricks. 
Exhausts enter the regenerator at temperatures from $2300^{\circ} \mathrm{F}$ to $2600^{\circ} \mathrm{F}$. Air preheat temperatures range from as low as $800^{\circ} \mathrm{F}$ for inefficient, one-pass designs to about $1600^{\circ} \mathrm{F}$ for more efficient one-pass designs or two- and fourpass designs (Webb 1982). Using secondary checkers, air preheat temperatures as high as $1100^{\circ} \mathrm{F}$ can be achieved.

Metallic recuperators are used on small, continuous melting furnaces (generally less than 100 tons/day) and on day tanks. They are not generally used on larger furnaces because the size required would be prohibitively expensive. There are two basic recuperator designs. One is an annular type, with hot gases flowing in the inner cylinder. The second design has a group of tubes arranged around the circumference of the flue, parallel to the waste gas flow (Webb 1982).

Metallic recuperators provide lower air preheat temperatures of about 1000 to $1400^{\circ} \mathrm{F}$. Their thermal effectiveness ranges from about $35 \%$ to $50 \%$ (Webb 1982). Expensive alloys, such as stainless steel and Inconel are required to withstand the high-temperature, corrosive waste gases.

Annealing

Annealing involves heating the glass after it has been formed to 1000$1200^{\circ} \mathrm{F}$ to reduce internal stresses, followed by controlled cooling to ambient temperatures. Annealing takes place in gas-fired lehrs. The lehr is a heated chamber in which the rate of cooling can be controlled. Annealing may consume as much as 10 percent of the total plant process heat. In flat glass manufacture, 500 to $1000 \mathrm{Btu} / \mathrm{ft}^{2}$ are required for annealing (Battelle 1976). Waste Heat Streams

Waste heat streams from glass manufacturing operations include exhaust gases from melting and annealing operations and contact and non-contact cooling water.

The theoretical enthalpy requirement for glass melting is $2.24 \times 10^{6}$ Btu/ton. Actual melting energy averages about $7 \times 10^{6}$ Btu/ton (A. D. Little 1976). This suggests that about $4.4 \times 10^{6} \mathrm{Btu} /$ ton is lost during glass melting. Based on an estimated 1980 production of $23 \times 10^{6}$ tons of glass, the annual waste heat from glass melting would be $101.2 \times 10^{12}$ Btu/year. Table E.18 provides an estimated heat balance for a typical melter. 
TABLE E.18. Melter Heat Balance

\begin{tabular}{|c|c|c|}
\hline & Btu/Short Ton & $\%$ \\
\hline Theoretical Heat Requirement & $2.24 \times 10^{6}$ & 32 \\
\hline Melter Exit Gases & $1.40 \times 10^{6}$ & 20 \\
\hline Radiation Losses & $\underline{3.40} \times 10^{6}$ & 48 \\
\hline Total & $7.04 \times 10^{6}$ & 100 \\
\hline
\end{tabular}

Radiation losses are barred from recovery due to practical considerations. These losses, however, can be reduced through better insulation practices or improved insulating materials.

The sources and temperatures of waste gases are listed in Table E.19. The average flow rate of gases from checker regenerators was found to be about $100,000 \mathrm{scf} /$ ton (AiResearch 1982).

Waste heat recovery from kiln exhausts is impeded by the fouling and corrosion potential of the waste streams. Potential foulants in glass melter exhausts include constituents from fuel combustion and from the batch, as well as degradation products from the refractory-lined furnaces and from the checkers.

TABLE E.19. Exhaust Gas Sources and Temperatures

\begin{tabular}{lr}
\multicolumn{1}{c}{ Source } & $\begin{array}{c}\text { Exit } \\
\text { Melting Furnace }\end{array}$ \\
Primary Regenerator & $2000-2800$ \\
Secondary Regenerator & $1200-1500$ \\
Metallic Recuperator & $800-1000$ \\
Annealing Lehrs & $1600-1800$ \\
& $1000-1200$
\end{tabular}

Because of the direct contact between the combustion gases and the glass batch in fuel-fired furnaces, the furnace exhausts are laden with particles and 
volatilized species from the glass. Gases bubble up through the melted glass and propel particles from the batch (Sittig 1977). These become entrained in the combustion gases which are flowing across the surface of the batch. The particulates consist primarily of sodium sulfate $\left(\mathrm{Na}_{2} \mathrm{SO}_{4}\right)$ from the salt cake added to the feed. Particulates al so include $\mathrm{Na}_{2} \mathrm{O}, \mathrm{SiO}_{2}, \mathrm{CaO}$ and cullet dust. The primary liquid component is sodium sulfate and $\mathrm{SO}_{3}$ (AiResearch 1980). The gas phase constiuents are primarily $\mathrm{NO}_{x}$ and $\mathrm{SO}_{2}$ (Sittig 1977; AiResearch, 1982).

Particulates collect in the regenerator and in the off-gas passages following the regenerator. The ultimate results of particulate deposition may include: (1) loss of regenerator effectiveness, (2) complete plugging of regenerator passages, or (3) destruction of regenerator brick. Particles collect within the checkers by impingement and settling, with impingement as the dominant mechanism (Sittig 1977). Dust and condensate collect on the surface of the bricks in the upper part of the checkers and form a slag that drips down into the lower zones. Here, because of the lower temperature, the slag solidifies and blocks the passages. Slag may also stay in the upper zones, causing plugging (Sittig 1977). The loss of effectiveness occurs as the pressure drop increases across the regenerator through constriction of the flow passages.

In the top zone of the regenerator, the following mechanisms are reported to contribute to erosion and corrosion of the brick (Kulkarni 1982):

- High-temperature flow of gases erode brick.

- MgO in magnesite brick reacts with $\mathrm{SiO}_{2}$ in the exhaust to form forsterite $\left(\mathrm{Mg}_{2} \mathrm{SiO}_{4}\right)$. This leads to volume expansion and subsequent crumbling of the brick.

- $\mathrm{MgO}$ and $\mathrm{CaO}$ combine with $\mathrm{SO}_{3}$ to form sulfates. The melting temperature of the bricks is lowered, leading to subsequent crumbling. This process is accelerated by the presence of vanadium oxide and sodium sulfate.

In the bottom zone of the regenerator, the following mechanisms are reported to lead to fouling and corrosion problems (Kulkarni 1982) $\cdot \mathrm{Na}_{2} \mathrm{O}$ 
and $\mathrm{SO}_{\mathrm{x}}$ combine to form sodium sulfate which condenses on the brick surface. The liquid sodium sulfate dissolves $\mathrm{SO}_{3}$ from the exhaust and it is transferred through the liquid to the refractory surface. The $\mathrm{SO}_{3}$ attacks the magnesium oxide (MgO) in the magnesite bricks and the calcium oxide ( $\mathrm{Ca} 0$ ) in the silicate bond to form magnesium sulfate $\left(\mathrm{MgSO}_{4}\right)$ and calcium sulfate $\left(\mathrm{CaSO}_{4}\right)$, respectively. Both reactions weaken the brick. These compounds form a eutectic mixture with the sodium sulfate, which drips down the bricks. When the gas flow is reversed, the eutectic is thought to form a white slag upon cooling. The sodium sulfate, still in the vapor phase, condenses when the gas flow is reversed to form a light powder.

Currently glass manufacturers do not employ secondary heat recovery equipment downstream from the checker regenerator. Potential fouling problems are the barrier to the adoption of secondary heat recovery equipment. Although much of the particulate carried over from the glass furnace collects in the regenerator, the exhausts leaving the regenerator still contain a high concentration of particulate. The composition of the particulates and the vapor contaminants leaving the regenerator are listed in Table E.20 for sodalime glass.

TABLE E.20. Constituents in Regenerator Exhausts (Webb 1982)

\begin{tabular}{|c|c|c|c|c|}
\hline & & Particulate $\mathrm{C}$ & Composition & (ppm) \\
\hline & & $\mathrm{Na}_{2} \mathrm{SO}_{4}$ & & 90 \\
\hline & & $\mathrm{K}_{2} \mathrm{SO}_{4}$ & & 3 \\
\hline & & $\mathrm{CaSO}_{4}$ & & 4 \\
\hline & & $\mathrm{SiO}_{2}, \mathrm{Al}_{2} \mathrm{O}_{3}$, & others & 3 \\
\hline Vapor & Comp & osition & Gas-Fired (ppm) & 0 il-Fired (ppm) \\
\hline & $\mathrm{SO}_{2}$ & & $10-80$ & up to 270 \\
\hline & $\mathrm{SO}_{3}$ & & $0-2$ & $2-10$ \\
\hline & ${ }^{N} 0_{x}$ & & 433 & 433 \\
\hline & $\mathrm{HCl}$ & & 13.7 & 13.7 \\
\hline & HF & & 0.1 & 0.1 \\
\hline
\end{tabular}


The following potential fouling problems in heat exchangers for secondary recovery have been reported for the hot end and the cold end of the exchanger (Webb 1982):

HOT END: - Corrosion may result from the reaction of $\mathrm{SO}_{3}$ with $\mathrm{K}_{2} \mathrm{SO}_{4}$ and $\mathrm{Na}_{2} \mathrm{SO}_{4}$, which form corrosive pyrosulfates, $\mathrm{K}_{2} \mathrm{~S}_{2} \mathrm{O}_{7}$ and $\mathrm{Na}_{2} \mathrm{~S}_{2} \mathrm{O}_{7}$, respectively.

- The liquid pyrosulfates entrap particulates.

- The pyrosulfates dry to a hard scale as the surface temperature is lowered. Scales are not removable by air blowing, but are water soluable.

COLD END: - If the surface temperature is below the dewpoint temperature, $\mathrm{H}_{2} \mathrm{SO}_{4}$ condenses. The condensed acid may react with sodium sulfate to form corrosive $\mathrm{Na}_{2} \mathrm{SO}_{7}$.

- If water condenses with the acid, $\mathrm{NaHSO}_{4}$ may be formed.

- The $\mathrm{Na}_{2} \mathrm{~S}_{2} \mathrm{O}_{7}$ and $\mathrm{NaHSO}_{4}$ form a sticky surface which traps particulates, forming a sludge.

Non-contact cooling water is used for equipment cooling. Cullet quench water is used to dissipate the heat from the scrap glass from product rejection, breakage or intentional wasting of molten glass during production interruptions. Cooling and process water for cullet quenching vary considerably between the product operations. Table E.21 summarizes the temperature above ambient, average flow rate and heat content of cooling and process waters by product category. 
TABLE E.21. Waste Heat Discharged in Cooling and Process Waters (Latour 1981)

$\begin{array}{lccc} & \begin{array}{c}\text { Flow } \\ \text { (Gal/Ton) }\end{array} & \begin{array}{c}\text { T } \\ \text { Flat Glass }\end{array} & \begin{array}{c}\text { Waste Heat } \\ \text { (Btu/Ton) }\end{array} \\ \text { Container Glass } & 500-20,000 & 10-40 & 2 \times 10^{6} \\ \text { Pressed and Blown Glass } & 1500 & 11 & 6 \times 10^{4} \\ \text { - Pressed and Blown } & 1350 & 18 & 2 \times 10^{5} \\ \text { - Glass Tubing } & 2000 & 8 & 1 \times 10^{5} \\ \text { - T. V. Tubes } & 3000 & 25 & 6 \times 10^{5} \\ \text { - Incandescent Lights } & 1080 & 14 & 1 \times 10^{5} \\ \text { - Frosting } & 950 & 20 & 1 \times 10^{5}\end{array}$

LIME - SIC 3274

In 1982, lime producers at 147 plants in the United States sold or used 14.6 million tons of lime. About $23 \%$ ( $3.3 \mathrm{million}$ tons) of this is captive lime (BuMines 1983). Captive lime is produced for consumption within a process rather than for sale.

Process Description

The lime production process is shown in Figure E.5. Basically, limestone is produced by calcining high-calcium or dolomitic limestone. The reactions that occur in the calcination step are given below for each type of limestone:

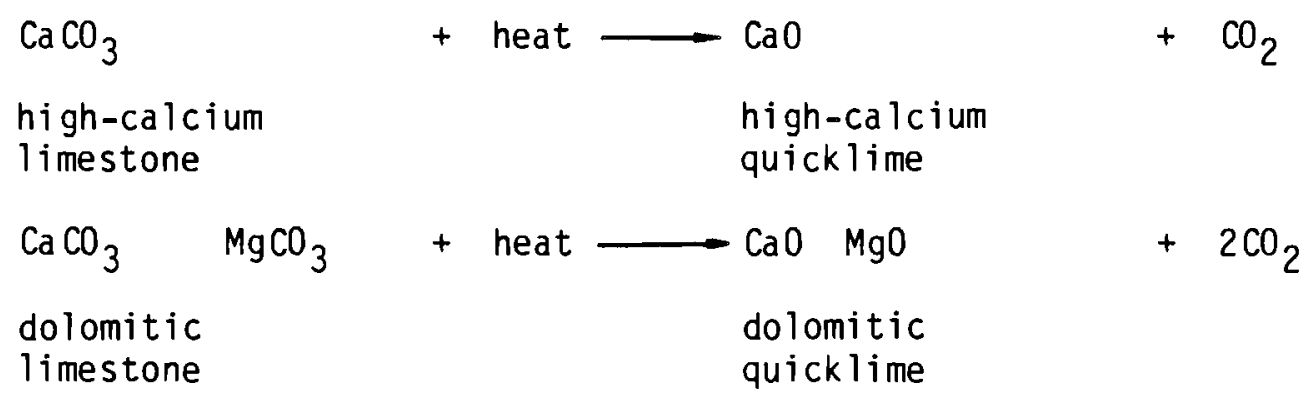




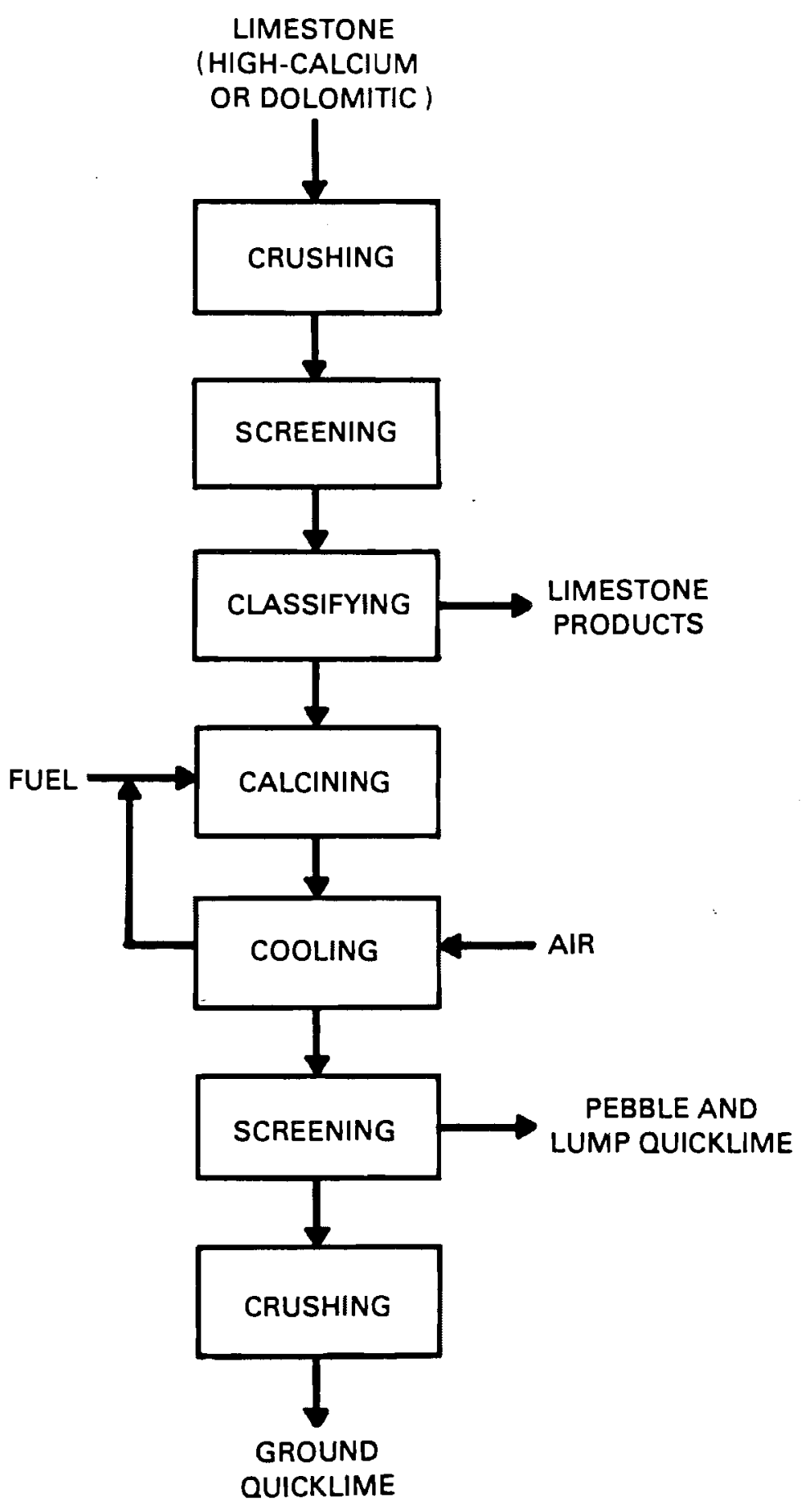

FIGURE E.5 Lime Production Process 
At temperatures between 1700 and $2450^{\circ} \mathrm{F}$, dissociation of limestone proceeds gradually from the outer surface of the particle toward the center. In general, larger diameter particles require higher calcining temperatures. Thus, feed crushing and screening have a significant effect on kiln energy use. Temperature control in the kiln must be maintained to obtain a highquality product. Insufficient temperatures result in incomplete calcination, which tends to dissipate the concentration of lime in the final product. Overheating the lime may reduce the reactivity of the lime through desensification and occlusion of surface micropores and fissures (Kirk-0thmer, 3rd Ed.).

The lime industry is energy-intensive, consuming about 7.1 million Btu/ton of lime productd (BuMines 1983). More than $70 \%$ of the fuel used in lime production is coal and about $25 \%$ is natural gas. The remaining $5 \%$ is fuel oil and other fuels. It is expected that the industry will continue to convert to coal within the limits of kiln fuel use capabilities.

Heat-Transfer Operations

The major heat-transfer operation in lime manufacture is calcining. Calcining accounts for 90 to $95 \%$ of the energy used in lime production (Warren 1980). Other heat-transfer operations include air cooling of calcined limestone and non-contact water cooling of equipment.

\section{Calcining}

Calcining is a direct-heat operation carried out at $2000^{\circ} \mathrm{F}$ to $2400^{\circ} \mathrm{F}$. A variety of refractory-lined kilns are used to calcine limestone. They vary with respect to capacity, energy use, and product-quality capability.

About $85 \%$ of the commercial and $70 \%$ of the captive lime is produced in rotary kilns (BuMines 1983). The production capacity of rotary kilns ranges from 50 tons/day to 1000 tons/day (Schwarzkopf 1970). Use of large rotary kilns in the cement industry suggests that it is possible to produce 3000 tons/day lime in a single rotary kiln system (Schwarzkopf 1970). Rotary kilns have three distinct advantages over other kiln systems: (1) they can use any type of fuel, (2) they produce a higher quality product, and (3) they have a larger capacity. Rotary kilns generally have a higher fuel consumption per 
unit of product and a higher capital cost than other types of kilns. Using a preheater with a rotary kiln is one means of reducing energy consumption. In the preheater, the exit gases from the kiln are mixed with the incoming feed and some surface calcination occurs.

Vertical kilns are available in capacities from 100 to 680 tons/day. These use less fuel than the rotary kilns per ton of output, but they are restricted to gaseous and liquid fuels. These kilns also produce a lower quality product. Because of their lack of fuel flexibility and lower-quality output, these kilns are being phased out.

Two other types of $k i l n s$ that have limited application in the United States are the rotary hearth calciner and the fluid bed. Table E.22 summarizes some of the pertinent features of the four types of kilns.

Lime Cooling

Calcined lime from the rotary kiln drops onto a cooler through which cooling air is passed. Coolers may be contact-, rotary- or grate-type coolers. The calcined lime is cooled from about $2000^{\circ} \mathrm{F}$ to $200^{\circ} \mathrm{F}$ (Intertechnology 1977). The cooling air is heated from ambient to about $1000^{\circ} \mathrm{F}$. The heated air serves as combustion air in the $k i l n$.

TABLE E.22. Comparison of Lime Calcining Systems (Schwarzkopf 1970; Intertechnology 1977)

\begin{tabular}{|c|c|c|c|c|c|}
\hline & Rotary & $\begin{array}{l}\text { Rotary with } \\
\text { Preheater }\end{array}$ & Vertical & $\begin{array}{l}\text { Rotary } \\
\text { Hearth } \\
\end{array}$ & $\begin{array}{c}\text { Fluid } \\
\text { Bed }\end{array}$ \\
\hline $\begin{array}{l}\text { Capacity } \\
\text { (tons/day) }\end{array}$ & $100-1000$ & $150-1000$ & $100-680$ & $100-600$ & $50-300$ \\
\hline Type of Fuel & any & any & gas & gas/oil 1 & gas/oi 1 \\
\hline $\begin{array}{l}\text { Fuel Use } \\
\text { (10 Btu/ton) }\end{array}$ & $6-12$ & $5-6$ & 5 & $4.7-5$ & 4.5 \\
\hline $\begin{array}{l}\text { Ower Use } \\
\text { kWh/ton) }\end{array}$ & $24-32$ & $32-36$ & $10-25$ & $28-32$ & 25 \\
\hline
\end{tabular}




\section{Waste Heat Streams}

Waste heat streams from lime manufacture include kiln exhausts and noncontact cooling water.

The theoretical enthalpy requirement for lime production is $4.23 \times 10^{6}$ Btu/ton for high-calcium quicklime and $3.85 \times 10^{6}$ Btu/ton for dolomitic quicklime (Kirk-0thmer, 3rd Ed.). Most of the lime produced is high-calcium. Comparison of the theoretical enthalpy requirement with the energy consumption figures presented earlier in Table E.22 shows that in practice a great deal more heat is required because of losses through the kiln shell, in the exhaust gases, and as sensible heat in the product. Table E.23 provides an estimated energy balance for a rotary lime kiln.

\section{TABLE E.23. Estimated Energy Balance for Rotary Lime Kiln}

$\begin{array}{lcc} & \frac{10^{6} \text { Btu/ton }}{4} & \frac{\%}{60} \\ \text { Theoretical Heat Requirement } & 4.23 & 23 \\ \mathrm{Kiln} \text { Exhaust } & 1.63 & 16 \\ \text { Product Sensible Heat } & 1.14 & \frac{1}{100} \\ \text { Power Use } & \frac{0.10}{7.10} & 100\end{array}$

Kilns with preheaters recover some of the heat rejected in the $k i$ in exhausts and some of the sensible heat in the product is recovered for combustion air preheat. The temperatures of kiln exhausts range from 1800$2000^{\circ} \mathrm{F}$. If a preheater is used, the exit gases from the preheater range from about $450^{\circ} \mathrm{F}$ to $550^{\circ} \mathrm{F}$ (Intertechnology 1977).

Kiln exhausts are laden with dust particles. The average dust loading in a rotary $k i \ln$ is reported to be $10 \%$ of the $k i l n$ feed or approximately 44 lbs/ton of lime, allowing for about 2 tons limestone/ton lime (Kirk-0thmer, 3rd Ed.). The hot combustion gases from direct-fired combustion blow the dust from the kiln. The dust is hot, dry, and difficult to wet. In addition, the 
particles are prone to becoming electrostatically charged (Sittig 1977). The dust varies in composition from raw limestone to calcined lime. It will also contain fly ash and unburned carbon if pulverized coal is the fuel.

Flow rates of water for non-contact equipment cooling are estimated to be 100 to 400 gallons/ton of lime produced. The discharged water ranges from $5^{0} \mathrm{~F}$ to $10^{\circ} \mathrm{F}$ above the inlet, corresponding to between 5000 and $30,000 \mathrm{Btu}$ of heat rejected per ton of lime produced (Latour 1981).

GYPSUM - SIC 3275

The primary uses of gypsum are in cement production and in the manufacture of plaster products used as building materials. Over $60 \%$ of the gypsum produced is used for building materials and plaster products (BuMines, 1983). Because of its association with the construction industry, gypsum manufacturing is somehat seasonal. About $60 \%$ of the industry energy requirement is consumed between April and September (Battelle 1976).

Process Description

Figure E.6 shows the processing steps in gypsum manufacture. Gypsum occurs as a relatively pure hydrated calcium sulfate, $\mathrm{CaSO}_{4} \cdot 2: \mathrm{H}_{2} \mathrm{O}$. The crude gypsum is crushed and ground in roller mills. Typically, drying occurs during grinding. About $40 \%$ of the gypsum is withdrawn after this step for sale as Portland cement retarder or agricultural gypsum.

Calcining converts the hydrate to the hemihydrate as follows:

$$
\mathrm{CaSO}_{4} \cdot 2 \mathrm{H}_{2} \mathrm{O} \longrightarrow \mathrm{CaSO}_{4} \cdot 1 / 2 \mathrm{H}_{2} \mathrm{O}+1-1 / 2 \mathrm{H}_{2} \mathrm{O}(\mathrm{g})
$$

A portion of the calcined gypsum is sold as gypsum building plaster following grinding and packaging.

The majority of calcined limestone is further processed to manufacture prefabricated forms. This involves extruding a layer of wet plaster on a moving sheet of cardboard, followed by covering with another sheet of cardboard. Initial setting and hardening is promoted through the use of additives. After setting, the sheets are cut and sent to the dryers. In the dryers, the moisture content of the plasterboard is reduced from about $50 \%$ to between 1 and 2\% (Battelle 1976). The dried plasterboard is then cooled and sent to storage. 


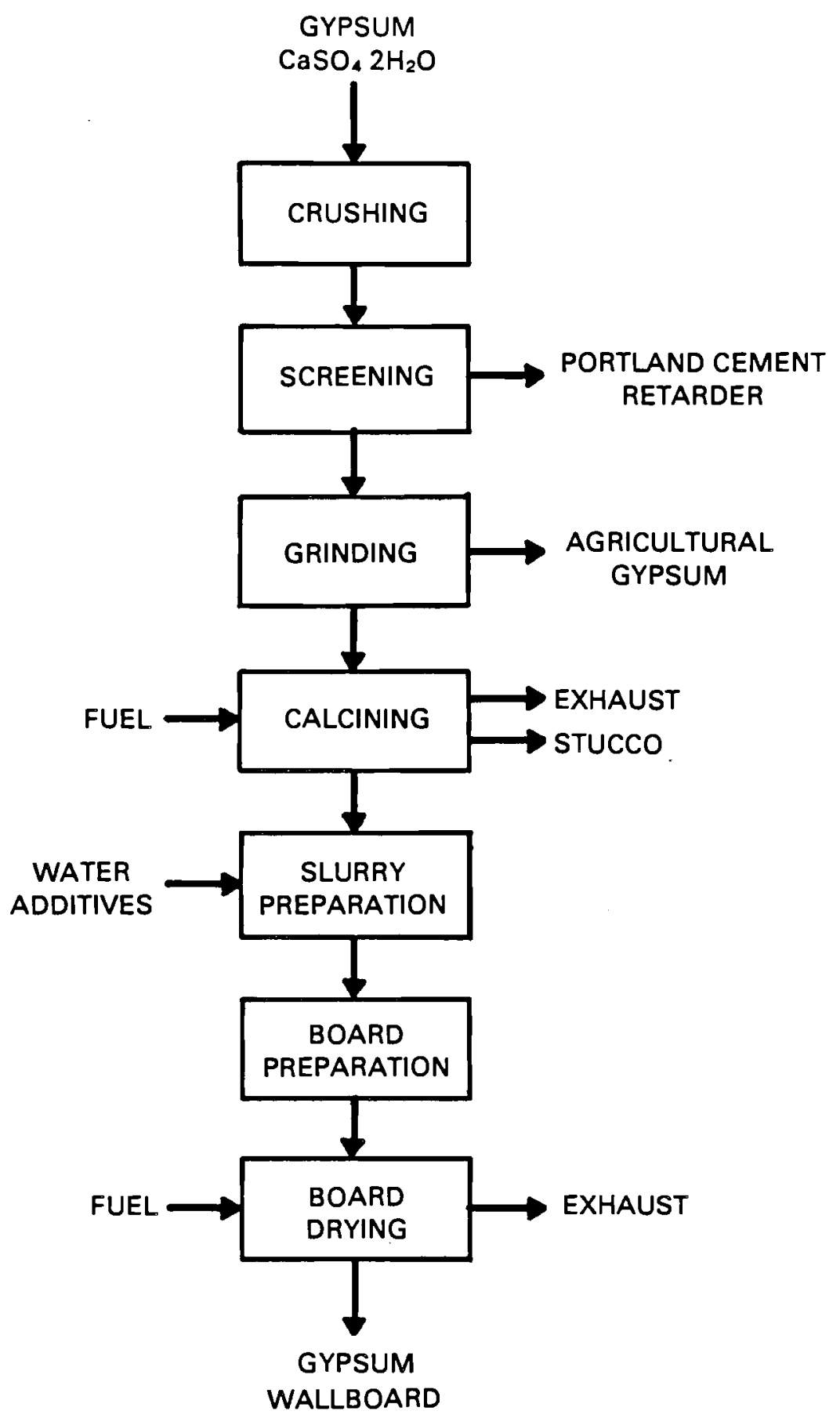

FIGURE E.6 Gypsum Processing 
The breakdown of energy use by the gypsum industry is as follows (BuMines 1980):

$\begin{array}{ll}\text { Natural Gas } & 82 \% \\ \text { Electricity } & 7 \% \\ \text { Fuel 0il No. } 2 & 5 \% \\ \text { Fuel 0il No. } 4 \& 6 & 3 \% \\ \text { Propane } & 2 \% \\ \text { Coal } & 1 \% \text {. }\end{array}$

Heat-Transfer Operations

The major heat-transfer operations in gypsum manufacture are calcining and plasterboard drying.

Gypsum Calcining

Most gypsum is calcined in large, direct-fired kettles. A few rotary kilns are used. The steel kettles are 8 to 15 feet in diameter and 6 to 14 feet deep (Battelle 1976). The kettles hold between 10 and 25 tons of gypsum (Shreve 1977). Ports are provided to charge the feed and withdraw the product.

A series of burners located under the kettle provide the heat for calcination. The hot combustion gases flow through the annular space between the kettle and a brick shell. In addition, a number of horizontal flues run through the kettle to enhance heat transfer (Intertechnology 1977). The gypsum is continuously stirred by several sweep arms or blades.

Calcining is a batch operation, requiring from one to two and one-half hours, depending on the batch size and firing rate. The batch is charged to the kettle and it is then fired. The water of hydration begins to be driven off at about $120^{\circ} \mathrm{F}$. The temperature then increases and is maintained at 250$260^{\circ} \mathrm{F}$ until calcination to the hemihydrate is completed. At this point, a rapid temperature rise occurs. When the temperature reaches $320-340^{\circ} \mathrm{F}$ the batch is withdrawn.

Between $0.8 \times 10^{6}$ and $1.1 \times 10^{6}$ Btu/ton of hemihydrate is used. Excess air ranges from 40 to $100 \%$ and overall efficiency is about $50 \%$ (Intertechnology 1977). 
Wallboard Drying

The moisture in the wallboard is removed by drying. Dryers usually contain several tiers and have two or three zones at different, but constant, temperatures. Typically, the temperature zones are as follows (Intertechnology 1977):

$\begin{array}{ll}\text { first zone } & 450^{\circ} \mathrm{F} \\ \text { second zone } & 440^{\circ} \mathrm{F} \\ \text { third zone } & 150-230^{\circ} \mathrm{F} .\end{array}$

Temperatures of the drying gases generally do not exceed $450^{\circ} \mathrm{F}$ to avoid warping and possible recalcination of the gypsum (Intertechnology 1977). Each zone is equipped with a fan and a heater for the air. Partial recycling of the air stream helps to reduce heat losses. Heat for the air heaters is most commonly supplied by steam, although the use of heat-transfer liquids is increasing (Battelle 1976).

Waste Heat Streams

The major waste heat streams from gypsum manufacturing are the exhaust gases from calcining and from wallboard drying.

The stack gases from calcining operations range from 700 to $1100^{\circ} \mathrm{F}$ (Inter-technology 1977). Apparentiy no heat recovery techniques are commonly employed to recover heat from these exhausts. Dryer exhausts are generally less than about $200^{\circ} \mathrm{F}$ and are high in moisture.

BRICK AND STRUCTURAL CLAY - SIC 3251

Common brick has historically accounted for most of the value of shipments for this industry. Minor products include ceramic glazed brick and tile and structural tile.

Process Description

Figure E.7 shows the major steps in brick manufacture. Clay from mines or quarries is crushed, ground and classified to obtain uniform particle diameters for feed materials.

There are three forming processes: (1) the soft-mud, (2) the stiff-mud, and (3) the dry-press. The stiff-mud process is now predominant (Shreve 1977). In the soft-mud process, the clay is mixed with water, excess water is 


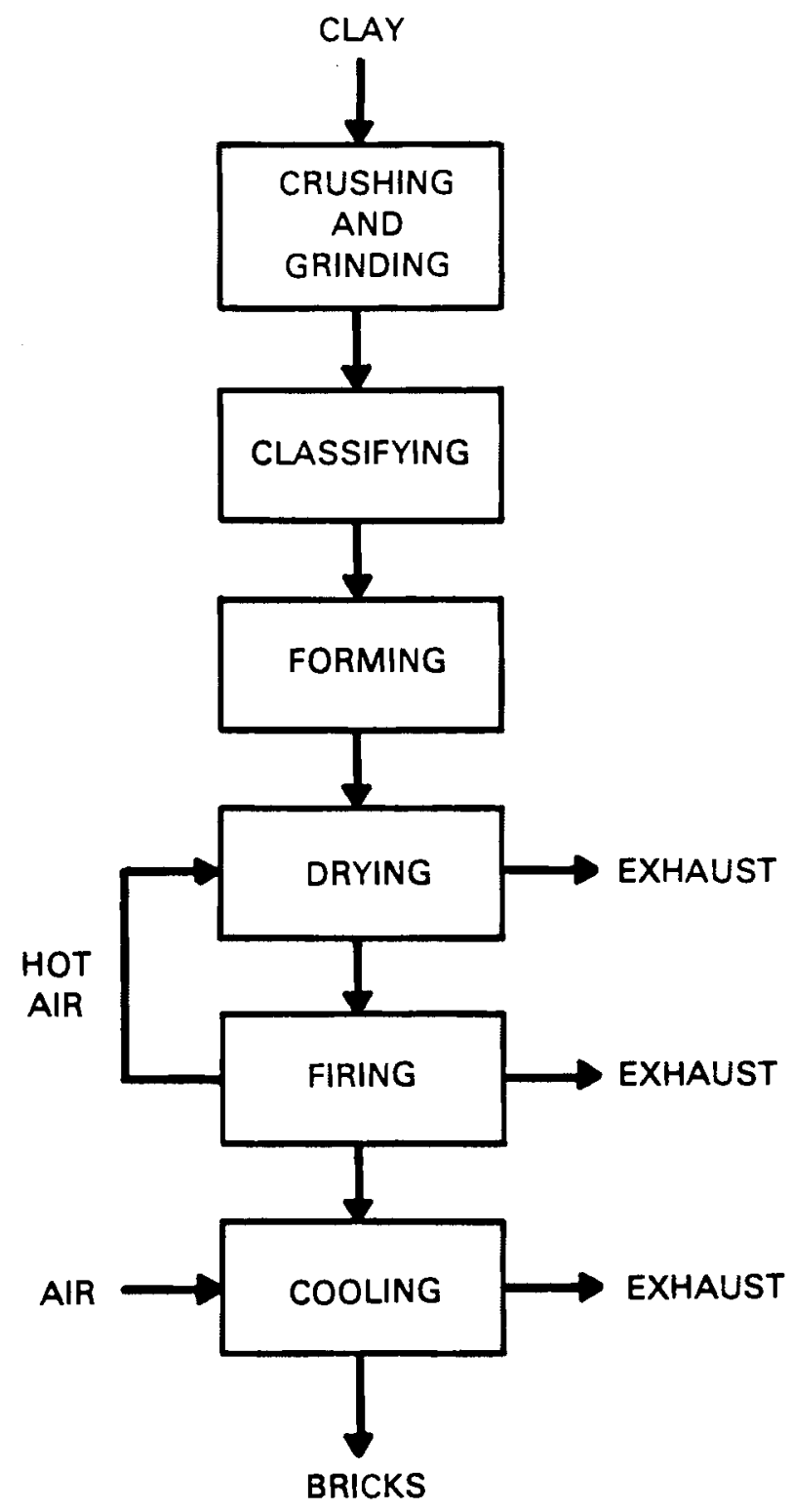

FIGURE E.7. Brick Manufacturing

filtered off (leaving 20 to $30 \%$ water), and the mixture is de-aerated to reduce porosity. The batch is then extruded into slugs which are soft and easy to form.

The stiff-mud process involves adding 12 to $15 \%$ water to the clay. The moistened clay is passed through a die and into a de-airing chamber in which a vacuum is maintained to remove gases in the clay. Finally, the mass is extruded continuously in columns, which are wire cut to the appropriate length. 
In dry pressing, only 3 to $4 \%$ moisture is added to the dry clay. A press that delivers about 5 tons $/ \mathrm{in}^{2}$ is used to mold the brick. This process is not commonly used.

Following forming, bricks are dried and then fired. Fired bricks are slowly cooled to avoid thermal shock. The combined drying and firing steps account for about $90 \%$ of the total energy consumed by the process (Battelle, 1976).

Heat-Transfer Operations

The major heat-transfer operations in brick manufacturing are drying and firing.

Drying

Historically, bricks were dried outside at ambient temperatures. However, most brick is now dried in ovens at temperatures between $300^{\circ} \mathrm{F}$ and $400^{\circ} \mathrm{F}$ (Intertechnology 1977). These dryers may be direct fired, steam heated, or resistance heated. A significant portion of the dryers use waste heat from kiln exhausts exclusively.

Firing

Most brick is fired in continuous tunnel kilns rather than in the older, less efficient batch kilns. Kiln capacity ranges from about 80 to 1000 tons/day (Intertechnology 1977). The trend toward replacing batch kilns with continuous tunnel kilns is expected to continue.

Kiln firing temperatures range from about 1800 to $2200^{\circ} \mathrm{F}$ (Warren 1980 ). Total process heat requirements for a kiln which also supplies all dryer heat requirements is about $3.3 \times 10^{6}$ to $3.4 \times 10^{6} \mathrm{Btu} /$ ton of product (Intertechnology 1977).

Waste Heat Streams

The most significant waste heat streams in brick manufacture are the exhaust gases from kilns and drying ovens. Exhaust temperatures from the kiln that are recycled to the dryers are between $300^{\circ} \mathrm{F}$ and $400^{\circ} \mathrm{F}$ (Intertechnology 1977 . 
APPENDIX $F$

TEXTILES - SIC 22 
. 
APPENDIX $F$

TEXTILES - SIC 22

The textile industry produces a wide variety of products from both natural and synthetic fibers. These products include knit and woven fabrics, rugs and carpets, and thread and yarn. In 1977, there were 7,202 textile mills in the United States (U.S. Department of Commerce 1981). The industry is concentrated in the Southeast, primarily in North and South Carolina and Georgia.

Textile plants range from highly integrated mills that process fibers into finished products to smaller, non-integrated plants that process goods owned by other producers on a contract basis. The industry has been classified according to the Standard Industrial Classification (SIC) as shown in Table F.1. Knitting mills constitute the largest group within the industry with 2,617 plants.

Within the textile industry, textile finishing (SIC 226) is the largest user of purchased fuels and energy. Energy purchases for 1980 are shown in Table F.2 by SIC number. Two-thirds of the energy consumed in the industry is

TABLE F.1. Textile Mill Industry Classification (U.S. Department of Commerce 1981)

SIC Number

22

2211

2221

2231

2241

225

226

227

228

229
Description

Textile Mill Products

Weaving Mills, Cotton

Weaving Mills, Manmade Fiber, Silk

Weaving and Finishing Mills, Wool

Narrow Fabric Mills

Knitting Mills

Textile Finishing, Except Wool

Floor Covering Mills

Yarn and Thread Mills

Miscellaneous Textile Goods
Number of Establ i shments

7,202

314

449

165

335

2,617

678

592

798

1,254

F. 1 
TABLE F.2. Textile Industry Energy Use, 1980

(Annual Survey of Manufactures 1980)

SIC Number

2211

2221

2231

2241

225

226

227

228

229
Industry Category

Weaving Mills, Cotton

Weaving Mills, Manmade Fiber, Silk

Weaving and Finishing Mills, Wool

Narrow Fabric Mills

Knitting Mills

Textile Finishing, Except Wool

Floor Covering Mills

Yarn and Thread Mills

Miscellaneous Textile Goods
Energy Use

(10 $12^{\mathrm{Btu})}$

35.9

44.9

4.9

3.7

52.8

65.4

27.9

35.5

23.9

used in fabric finishing (Streb 1977). Most of the energy is used to raise steam, which is used for drying, and for heating water for washing or chemical preparation. Between 50 and $70 \%$ of the fossil energy consumed by the textile industry is used for steam production (Georgia Institute of Technology 1982). Direct firing of natural gas in dryers and ovens is the second largest use of energy, accounting for $25 \%$ of the total energy use.

Textile production consists of two basic operations: (1) the production of undyed, unfinished (greige) fabric, and (2) the production of finished goods from the greige fabric.

\section{PROCESS DESCRIPTION}

Figure F.1 shows typical processing steps for knit and woven greige goods production. These front-end operations are essentially dry, with the exception of slashing.

Yarn preparation converts the natural or synthetic fibers to yarn. Yarn which is to be used in knitting operations is treated with an oil or wax 


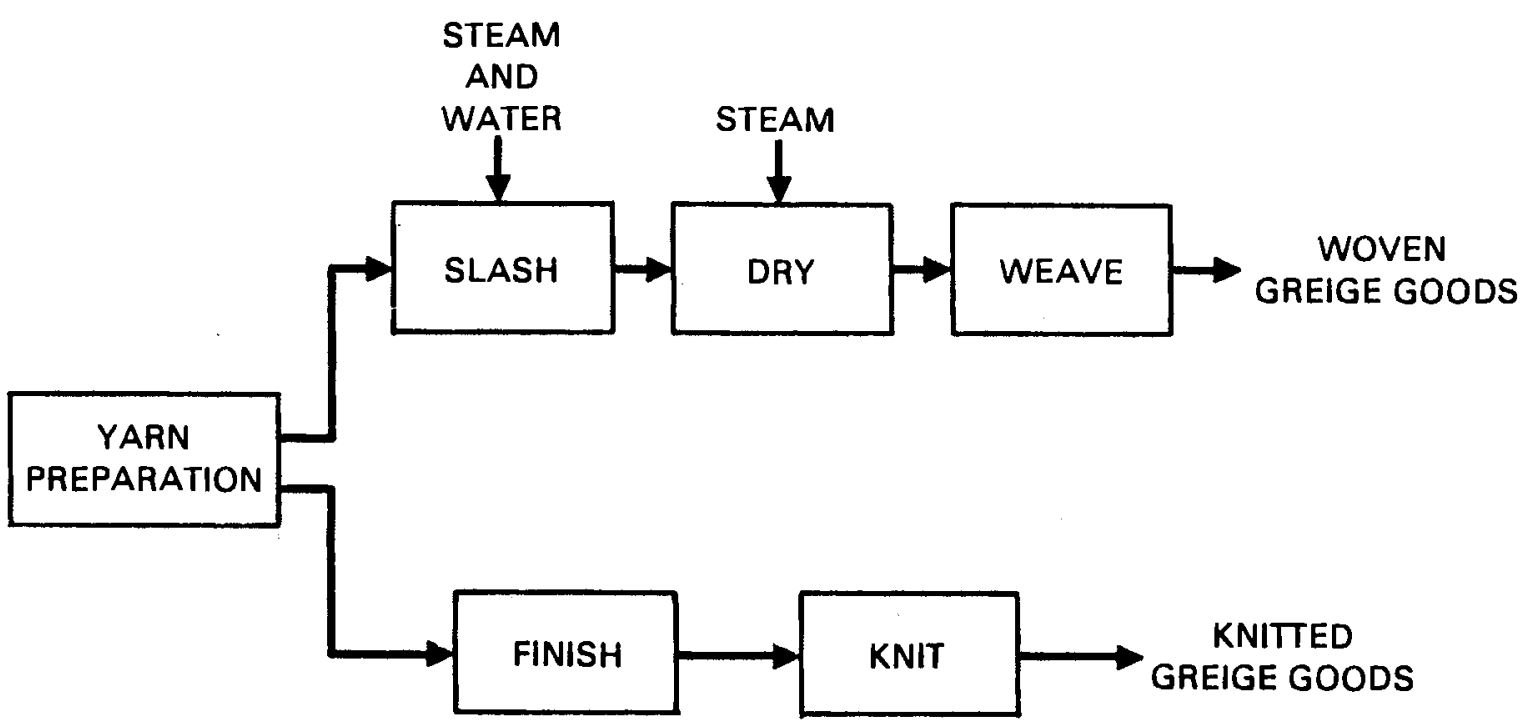

FIGURE F.1 Typical Processing Steps for Knit and Woven Greige Goods Production

emulsion in the finishing process. This treatment helps to increase the speed of the knitting process.

Yarn destined for weaving mills is treated with compounds such as carboxymethylcellulose, polyvinyl alcohol, or starch in the slashing (sizing) process. These compounds protect the thread against abrasion during weaving. The size compounds are applied by passing the yarns through a hot aqueous solution containing the sizing chemicals. The solution is generally heated through direct steam injection. Following sizing, the thread is dried by passing it over steam-heated drying cylinders. The thread is then sent to the weaving looms for production of woven greige fabric.

Finishing operations (dyeing and finishing) transform the greige goods into finished fabric. Figure F.2 shows the typical processing steps in finishing woven and knit greige fabric. Many of the finishing steps are wet operations which use water heated by direct steam injection. Textile mills use a great deal of process water for product washing, air-washing, boiler makeup, cooling of process equipment, and as a dilutent for process chemicals. Drying operations use direct-fired natural gas or indirect steam heating of drying cyl inders. 

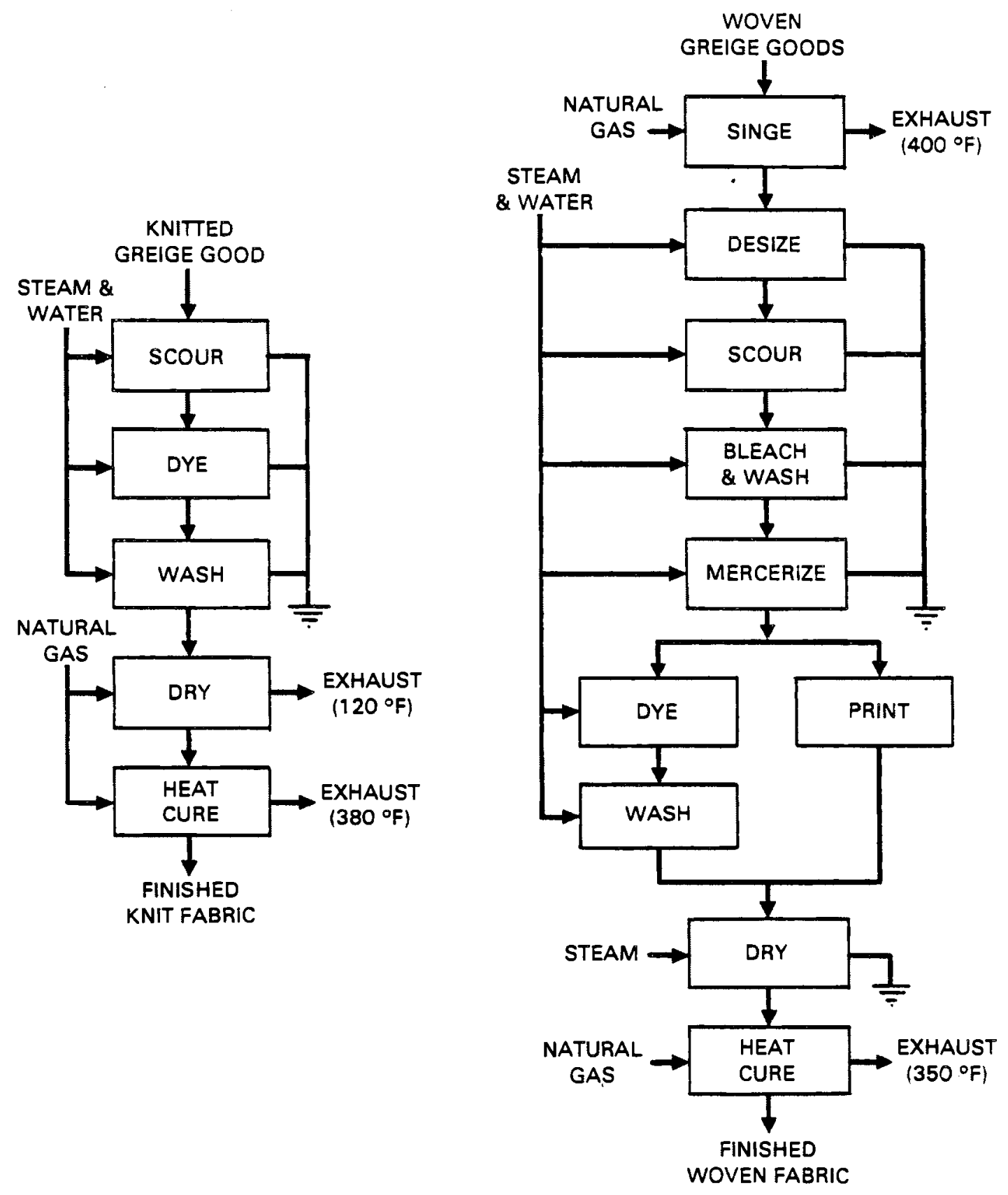

FIGURE F.2 Typical Processing Steps in Finishing Woven and Knit Greige Fabric 
HEAT-TRANSFER OPERATIONS

The major heat-transfer operations in textile production are listed in Table F.3. An operation is designated as "non-contact" if the heat-transfer fluids do not mix with the media that is being heated and as "contact" if they are in contact.

Steam Generation

Steam process heat demands from plant-to-plant range from 4000 to 40,000 lbs/hr (Battelle 1976). The steam temperature at the boiler is in the range of 250 to $400^{\circ} \mathrm{F}$ (Battelle 1976). Gas and oil-fired boilers have largely been converted back to coal, with standby capability provided by fuel oil or natural gas. Several textile plants now purchase steam from a central source, rather than generate steam on-site (Power 1983). For all greige mills, steam pressures range between $15 \mathrm{psig}$ and $125 \mathrm{psig}$. Integrated mills generally use steam between 100 and 150 psig (UTC 1980).

Drying (Drying Cylinders)

Steam-heated drying cylinders (drying cans) are used for drying both in woven greige goods production and in fabric finishing. In greige goods production, the treated warp yarns from the sizing operation are passed over the drying cylinders prior to weaving. Drying cans are used for predrying fabric prior to drying in tenter-frame dryers.

TABLE F.3. Textile Mill Heat-Transfer Operations

\begin{tabular}{lllll} 
& \multicolumn{1}{c}{ Operation } & \multicolumn{1}{c}{ Equipment } & & \multicolumn{1}{c}{$\begin{array}{c}\text { Heat-Transfer } \\
\text { Medium }\end{array}$} \\
\cline { 2 - 2 } NON-CONTACT: & $\begin{array}{l}\text { Steam Generation } \\
\text { Drying }\end{array}$ & Boiler & Combustion Gases \\
CONTACT: & Singeing & Drying Cans & & Steam \\
& Drying & Ovens & Natural Gas Flame \\
& Water and & Tenter Frames & & Combustion Gases \\
& Air Tempering & Injection Nozzle & Steam \\
& & Air Washers and & Chilled or Hot
\end{tabular}


Heat is transferred to the yarn or the fabric via conduction through the cylinder to the yarn. Steam is used at pressures ranging from 15 to 35 psig and from 75 to 125 psig, with the higher pressures used to increase the production rate (UTC 1980). The steam pressures are sufficient to keep the can surface temperature at about $315^{\circ} \mathrm{F}$ (Cook October 1978). About 2000 Btu are used in steam drying cans per pound of water evaporated (Hendrix 1983). Steam is condensed and returned to the boiler.

\section{Singeing}

Singeing is the process by which protruding fibers from yarn or fabric are burned off by passing over an open flame. Some fabrics are singed only on one side and some are singed on both sides.

Natural gas is burned to provide the flame for singeing. It is estimated that about $200 \mathrm{Btu} / 1 \mathrm{~b}$ of fabric is used in the singeing process (Cook January 1978).

Drying (Tenter Frames and Ovens)

Convective drying of fabric occurs as it moves through the tenter-frame dryers. Dryers are of two general types that differ in air flow arangement:

(1) flow-through dryers -- hot air is forced through the fabric from one side

(2) impingement dryers - high-velocity air is directed toward both surfaces of the fabric.

Most dryers have several zones with individual burners. Each zone has a separate temperature controller and exhaust duct. Table F.4 provides energy consumption information collected for several different dryers. The average energy use figure from this sample was determined to be about 2100 Btu/1b of fabric. A figure of 3000 Btu has been reported for tenter-frame dryers per pound of water evaporated (Hendrix 1983).

\section{Water or Chemical Heating}

Water and textile chemical heating may occur through direct or indirect heat transfer with steam. Examples of unit operations that require steam heating are slashing, dyeing, washing, and mercerizing. 
TABLE F.4. Dryer Performance Values (Cook January 1978)

\begin{tabular}{|c|c|c|c|c|c|c|}
\hline Sample & Fuel & $\begin{array}{r}\text { Fabric } \\
\text { Weight } \\
\text { lbs./yd. }\end{array}$ & $\begin{array}{l}\mathcal{X} \text { Moisture } \\
\text { Entering }\end{array}$ & $\begin{array}{c}\text { Highest } \\
\text { Exhaust Temp. } \\
{ }^{\circ} \mathrm{F} \\
\end{array}$ & $\begin{array}{c}\text { Exhaust } \\
\text { Humidity } \\
\text { Ratio } \\
\end{array}$ & $\begin{array}{c}\text { Energy } \\
\text { Requi red } \\
\text { Btu/lb. }\end{array}$ \\
\hline IA & Propane & 2.96 & 129 & 247 & .04 & 2,690 \\
\hline 18 & Propane & 2.76 & 122 & 210 & .04 & 2,250 \\
\hline $2 A$ & Steam & 2.12 & 26 & 254 & .015 & 1,800 \\
\hline 2B & Steam & & 22 & 254 & .015 & 1,400 \\
\hline $3 A$ & Steam & .534 & $161 / 2$ & 193 & .012 & 2,880 \\
\hline $3 B$ & Steam & 2.19 & 55 & 220 & .03 & 1,610 \\
\hline 4 & Propane & .77 & 30 & 300 & .04 & 1,075 \\
\hline 5 & & & & & & \\
\hline 6 & Natural Gas & & 35 & 320 & .04 & 1,160 \\
\hline 7 & Natural Gas & .85 & 77 & 380 & .03 & 3,150 \\
\hline 8 & Natural Gas & .85 & 29 & 350 & .03 & 2,460 \\
\hline 9 & Natural Gas & .64 & 50 & 408 & NA & 1,220 \\
\hline 10 & Natural Gas & .53 & 50 & 400 & NA & 2,750 \\
\hline
\end{tabular}




\section{Air Tempering}

Ambient temperature and humidity control is extremely important in yarn and fabric processing. These variables are controlled by large, air-tempering systems. The textile industry is one of the largest users of air-washing equipment to clean and temper air in the processing areas. Tempering requires adding heat in the winter and removing heat in the summer. Chilled water is used in the air washers in the summer at temperatures of 40 to $50^{\circ} \mathrm{F}$ (Kemmer 1979). Heat collected in the chilled water is dissipated through evaporative cooling in cooling towers.

In the air tempering system, airborne particles (particularly lint) can build up on heat-transfer surfaces, reducing effectiveness. Lint can enter the cooling water loop through the natural scrubbing effect of the cooling tower, which tends to entrain airborne particulates (Capper 1974). Chiller tube sheets are vulnerable to fouling and corrosion. Dirt, fiber, oil, and microbiological deposits can form on tube sheet surfaces, impeding heat transfer. Severe pitting can occur under the slimes (Kemmer 1979).

\section{WASTE HEAT STREAMS}

Sources and approximate temperatures of waste heat streams from textile operations are summarized in Table F.5. Table F.6 provides flow quantities for a typical interated knit mill and an integrated weaving mill.

Exhaust yases from boilers may contain fly ash, unburned hydrocarbons, and $\mathrm{SO}_{3}$, which may condense at lower temperatures. The volume and enthalpy of the exhaust depends on the firing rate of the boiler, the amount of excess air, and the efficiency of primary and secondary heat-recovery equipment. It is difficult to generalize because of the variability across the industry.

Exhausts from singeing and drying operations contain lint, and exhausts from heat setting operations contain finishing chemicals as well as lint. Two major barriers to the adoption of heat-recovery equipment for textile dryers and ovens are the buildup of lint and the condensation of finishing chemicals on heat exchanger surfaces (Georgia Institute of Technology 1982). Finishing chemicals include anti-statics, fire retardants, softeners, water repellants, 
TABLEF.5. Sources of Waste Heat from Textile Mil1 Operations

\begin{tabular}{|c|c|c|}
\hline Type & Source & $\begin{array}{c}\text { Temperature } \\
\left({ }^{F}\right) \\
\end{array}$ \\
\hline \multirow[t]{3}{*}{ Exhaust Gas } & Boilers & $400-650$ \\
\hline & Singeing Ovens & $390-400$ \\
\hline & Tenter-Frame Dryers & $250-400$ \\
\hline Process Water & $\begin{array}{l}\text { Slashing, Bleaching, } \\
\text { Washing, Mercerizing, } \\
\text { Dyeing }\end{array}$ & $100-120$ \\
\hline Non-Contact Cooling Water & Product Cooling & 140 \\
\hline
\end{tabular}

TABLE F.6. Flow Rate of Waste Heat Streams (A. D. Little 1976) INTEGRATED KNIT MILL $(22,000$ ibs/day knit fabric $):$

\begin{tabular}{|c|c|c|}
\hline Type & Source & Flow Rate \\
\hline $\begin{array}{l}\text { non-contact cooling } \\
\text { water }\end{array}$ & dyeing operations & $114,000 \mathrm{gal} / \mathrm{day}$ \\
\hline process water & washing & $136,000 \mathrm{gal} /$ day \\
\hline flue gas & drying & $586 \times 10^{6} \mathrm{ft}^{3} / \mathrm{day}$ \\
\hline & heat setting & $5.78 \times 10^{9} \mathrm{ft}^{3} / \mathrm{day}$ \\
\hline
\end{tabular}

INTEGRATED WEAVING MILL $(66,000$ 1bs/day woven fabric):

$\begin{array}{lll}\text { flue gas } & \text { single \& heat set } & 17.7 \times 10^{12} \mathrm{ft}^{3} / \mathrm{day} \\ & \text { drying \& setting } & 18.7 \times 10^{12} \mathrm{ft}^{3} / \mathrm{day}^{2} \\ & \text { heat cure } & 17.5 \times 10^{12} \mathrm{ft}^{3} / \mathrm{day} \\ \text { process water } & \text { fabric preparation } & 1.11 \times 10^{6} \mathrm{gal} / \mathrm{day} \\ & \text { fabric dyeing } & 1.11 \times 10^{6} \mathrm{gal} / \mathrm{day}\end{array}$

or durable-press resins (Cooper 1978). These chemicals act as adhesives to hold the lint on the surface.

A heat recovery system with glass rather than metal tubes has been developed for use with dryer exhausts. The smooth, nonporous surface is more effective than metal tubes in preventing deposition of finishing chemicals or 
lint. In addition, the glass tubes are assembled in modules that can be easily removed for cleaning or replacement. The manufacturer recommends cleaning at six-month intervals. Cleaning takes approximately 12 hours in a heated tank with a circulating pump that is part of the system package (Georgia Institute of Technology 1982).

Another heat-recovery system that may mitigate the lint and finishingchemical deposit problems consists of two regenerator units that attach to the dryer. The dryer exit gas passes through one regenerator that stores heat from the exhaust while the cool, makeup air is heated as it passes through the opposite regenerator. Flow direction is alternated through the regenerators at set intervals. Deposition problems are minimized because lint builds up at the hot end of the regenerator and the finishing chemicals do not condense until they reach the cold end. The lint can then be easily removed by blowing with air. Weekly air cleaning is recommended (Georgia Institute of Technology 1982).

A large portion of the energy and water used in wet processing operations is used in batch operations. Thus, the waste heat rejected from these operations is not continuously available. Batch operations which reject low temperature waste heat in process water include various types of dyeing. Atmospheric dye becks $(a)$ reject about all of their input energy as waste heat. A heat balance for a typical atmospheric dye beck is given in Table F.7. The process water from dyeing contains unspent dye chemicals and lint.

(a) A vessel for drying fabric.

TABLE F.7. Heat Balance for Atmospheric Dye Beck (Cook January 1978)

$\begin{array}{lr} & \frac{\text { Percent }}{2} \\ \text { Radiation and Convection } & 49 \\ \text { Exhaust } & \frac{49}{100} \\ \text { Process Water } & \text { Total }\end{array}$


A. D. Little. 1976. Environmental Considerations of Selected EnergyConserving Manufacturing Process Options: Volume IV Petroleum Refining Industry Report, Cambridge, Massachusetts.

A. D. Little. 1976. Environmental Considerations of Selected Energy Conserving Manufacturing Process Options: Volume VPulp and Paper, Cambridge, Massachusetts.

A. D. Little. 1976. Environmental Considerations of Selected Energy Conserving Manufacturing Process Options: Volume VI Olefins Industry Report. PB-264-275, Cambridge, Massachusetts.

A. D. Little. 1976. Environmental Considerations of Selected Energy Conserving Manufacturing Process Options: Volume VII Ammonia Industry Report. PB-264 273, Cambridge, Mas sachusetts.

A. D. Little. 1976. Environmental Considerations of Selected Energy Conserving Manufacturing Process Options: Volume IX Textile Industry Report. PB-264 275, Cambridge, Massachusetts.

A. D. Little. 1976. Environmental Considerations of Selected Energy Conserving Manufacturing Process Options: Volume XI GTass Industry Report. PB-264 277, Cambridge, Massachusetts.

A. D. Little. 1976. Environmental Considerations of Selected Energy Conserving Manufacturing Process Options: Volume XII Chlor-Alkali Industry Report. PB-264 27, Cambridge, Massachusetts.

A. D. Little. 1976. Environmental Considerations of Selected Energy Conserving Manufacturing Process Options: Volume XIII Phosphoric Acid Industry Report. PB-264 279, Cambridge, Mas sachusetts.

A. D. Little. 1976. Environmental Considerations of Selected Energy Conserving Manufacturing Process Options: Volume XV Fertilizer Industry Report. PB-264 281, Cambridge, Massachusetts.

"Annual Refining Survey". March 22, 1982. 0 il \& Gas Journal.

Anonymous. 1983. "Industry Focuses on Reorganization." Power. 127(9):138140.

Babcock and Wilcox. 1975. Steam - Its Generation and Use. New York, New York.

Battelle Columbus Laboratories. 1976. Developing a Maximum Energy Efficiency Improvement Target for Sic 28: Chemicals and Allied Products. Columbus, ohio. 
Battelle, Columbus Laboratories and Battelle, Pacific Northwest Laboratories. November 1976. Survey of the Applications of Solar Thermal Energy Systems to Industrial Process Heat, Volume 2 - Industrial Process Heat Survey. Columbus, ohio.

Bhatty, M. S. Y. 1983. "Cement Kiln System Buildups." Proceedings of the 16th International Cement Seminar, Chicago, Illinois.

Bhatty, M. S. Y. 1983. "Composition of Waste Heat Boiler Buildups in Cement Kiln Systems." ASME Thermal Engineering Joint Conference 1983, Volume I. Honolulu, Hawai i.

Bland, William F. and Robert L. Davidson. 1967. Petroleum Processing Handbook. McGraw-Hi11 Book Company, New York, New York.

Bodine, J. F and M. Vitul10. 1980. Industrial Energy Use Data Book ORAU160, Oak Ridge Associated Universities, Oak Ridge, Tennessee.

Bureau of Census. 1980. 1980 Annual Survey of Manufactures: Fuels and Electric Energy Consumed. M80(AS)-4.1. U.S. Department of Commerce, Washington, D.C.

Bureau of Mines. 1980. Mineral Facts and Problems. Bulletin 671, U.S. Department of Interior, washington, D.C.

Bureau of Mines. 1983. Mineral Commodity Summaries. U.S. Department of the Interior, Washington, D.C.

Capper, C. G. 1974. "Fouling of Heat Exchangers from Cooling Water and Process Materials." Effluent and Water Treatment Journal.

Census of Manufactures. 1977. Industry Statistics, Volume II, Part 1. U.S. Department of Commerce, Washington, D.C.

Charles A. Berg Associates. 1982. Industrial Electrification Potential: Case Studies of the Primary Metals and Glass Industries. EPRI EA-2761, Prepared for Electric Power Research Institute, Pa To Alto, California.

Cook, F. L., et al. 1978. Energy Conservation in the Textile Industry, Phase I. OR0-5099-T1, prepared for the U.S. Department of Energy, Washington, D.C.

Cook, F. L., et al. 1978. Energy Conservation in the Textile Industry, Phase I I. OR0-5099-T2, prepared for the U.S. Department of Energy, Washington, $\overline{\mathrm{D} . \mathrm{C}}$.

Cooper, S. G. 1978. The Textile Industry: Environmental Control and Energy Conservation. Noyes Data Corporation, Park Ridge, New Jersey.

Drexel University. 1980. Energy Analysis of 108 Industrial Processes: Industrial Applications Study. Philadelphia, Pennsylvania. 
Electric Power Research Institute. 1982. Evaluation of Industrial Advanced Heat Recovery/Thermal Energy Storage Systems, Volume 2. EPRI EM-2573, Palo ATto, California.

Energy and Environmental Analysis, Inc. February 1983. Industrial Energy Productivity Project Final Report - The Petroleum Refining Industry. DOE/CS/40151-1, Vol. 3 of 9. Arlington, Virginia.

Energy Research and Development Administration. 1977. Upgrading Existing Evaporators to Reduce Energy Consumption. C00/2870-2, National Technical Information.Service, Springfield, Virginia.

Farrar, Gerald L., Editor. Questions and Answers on Refining Technology 19751979. National Petroleum Refiners Association, Washington, D.C.

Federal Energy Administration. 1974. Project Independence Blueprint-Final Task Force Report--Energy Conservation in the Manufacturing Sector, 1954-1990. Volume 3, Washington, D.C.

Gaines, L. L., et al. 1980. Energy and Materials Flows in the Production of 0lefins and The ir Derivatives. ANL/CNSV-9, Argonne National Laboratory, Argonne, Illinois.

Garrett AiResearch Manufacturing Company. 1982. Glass Furnace Recuperator Laboratory Development Program. Prepared for the U.S. Department of EnergyIdaho Operations, Idaho Falls, Idaho.

Garrett, H. M. 1980. "Coal and the Cement Industry." Mining Engineering pp. 188-198.

Garrett, H. M. and J. A. Murray. 1974. "Improving Kiln Thermal Efficiency." Rock Products, Reprint. Maclean Hunter Publishing Corportion, Chicago,

Tlinois.

Gartner, E. M. and S. W. Tresouthick. 1980. "Potential for Energy Conservation in the U.S. Cement Industry." World Cement Technology 13(5).

General Accounting Office. June 1980. How the Petroleum Refining Industry Approaches Energy Conservation - A Case Study.

Georgia Institute of Technology. 1982. Energy Conservation in the Textile Industry: 10 Case Histories. PNL-4363, washington, D.C.

Gilbreath, K. 1980. "A Balance of Water Conservation and Energy Recovery". TAPPI Engineering Conference 1980.

Grogan, P. J. 1981. Economic Analysis of Industrial Waste-Heat Recovery: Fluidized-Bed Technology Versus Three Conventional Alternatives. ANL/CNSV-TM66. Argonne National Laboratory, Argonne, Illinois.

Hamel, B. B. and H. L. Brown. 1979. Energy Analysis of 108 Industrial Processes. Drexel University. Philadelphia, Pennsylvania. 
Haynes, V. 0. September 1976. Energy Use in Petroleum Refineries. ORNL/TM5433. Oak Ridge National Laboratory. Oak Ridge, Tennessee.

Hendrix, W. A. 1983. "Research Needs and Priorities for Textile Process Electrification." Georgia Tech Engineering Experiment Station, Atlanta, Georgia.

Hersh, H.N. 1981. Energy and Material Flows in the Production of Pulp and Paper. Technical Report No. ANL/CNSV-16, Argonne National Laboratory, Argonne, Illinois.

Hogeland, A. 1983. Analyzing Heat-Recovery Projects for Optimum Energy Use. pp 63-65, Plant Engineering.

Howlett, C. and H. Garrett. 1981. "Precalciners Today." Presented at the IEEE Cement Industry Technical Conference, Lancaster, Pennsylvania.

International Research and Technology Corportion. 1974. Industrial Energy Study of the Industrial Chemicals Group (Final Report). IRT-342-R, Arlington, Virginia.

Intertechnology Corporation. 1977. Analysis of the Economic Potential of Solar Thermal Energy to Provide Industrial Process Heat, Final Report, Volume III. COO-28293.

Jansen, J. H. 1979. "Operation Parameters of a Black Liquid Chemicals and Heat Recovery Boiler". In ISA Transactions. 18:1(87-93).

Kemmer, F. N. (ed). 1979. The NALCO Water Handbook. McGraw-Hill Book Company, New York, New York.

Kirk-Othmer Encyclopedia of Chemical Technology. Third Edition, Wiley, New York, New York.

Kozureck, M. 1983. "Introduction to Pulp and Paper Technology" - Short Course, Atlanta, Georgia.

Kulkarni, A. K., et a1. 1982. "Fouling and Corrosion in Glass Furnace Regenerators" Proceedings of the International Conference on Fouling of Heat Exchange Surfaces. White Haven, Pennsylvania, Oct. 31-Nov. 5, 1982.

Lane, S. 1979. "Energy Reduction in a New Lime Kiln and Recausticsying

Plant." Tappi Engineering Conference Proceedings, 1979, Bk. 1.

Latour, S. R. and J. G. Menningman. 1981. Waste Heat Recovery Potential in Selected Industries. Prepared for Environmental Protection Agency, Cincinnati, Ohio.

Marner, W. J. 1982. An Assessment of Gas-Side Fouling in Cement Plants. DOE/ID-12138-2, Jet Propulsion Laboratory, Pasadena, California. 
McDonald, R. G., and J. N. Franklin. 1969. Pulp and Paper Manufacture Volume I: The Pulping of Wood. McGraw-Hill, New York, New York.

Mellon Institute. 1983. Industrial Energy Productivity Project - Final: The Pulp and Paper Industry. DOE Report No. DE83 00691, Pittsburgh, Pennsylvania.

Midland-Ross. 1983. Energy Conservation in the Paper Industry. Canada.

Minnesota Energy Agency. 1979. Minnesota Guide to Waste Heat Recovery for Industrial, Commercial and Institutional Organizations. PB80-150576, St. PauT, Minnesota.

Nelson, W. L. 1969. Petroleum Refinery Engineering. McGraw-Hill Book Company, New York, New York.

Nydick, S. E., et a 1. June 1976. A Study of Inplant Electric Power Generation in the Chemical, Petroleum Refining, and Paper and Pulp Industries. FEA/D76/321. Thermo Electron Corporation. WaTtham, Massachusetts.

Pacific Northwest Laboratory. 1980. Ten Case History Studies of Energy Efficiency Improvements in Pulp and Paper Mills. National Technical Information Service, Springfield, VA.

Palmer, W. B., H. E. Gerlaugh, and R. R. Priestley. April 1980. Cogeneration Technology Alternatives Study (CTAS), General Electric Company Final Report, Volume III - Industrial Processes. D0E/NASA-0031-80/3. General Electric Company, Schenectady, New York.

Pierce, D. P. et al. 1973. Emissions froma Kraft Recovery Boiler - the Effects of Operational ValuabTes. Babcock and Wilcox, Barberton, Ohio.

Portland Cement Association. 1980. U.S. and Canadian Portland Cement Industry: Plant Information Summary. Stokie, MTinois.

Post's Pulp and Paper Directory. 1982. Ed. James Ledbetter, Miller Freeman, New York.

Radian Corporation. 1980. Energy Use Patterns and Environmental Implications of Direct-Fired Industrial Processes. EPA-600/7-81-132, prepared for Industrial Environmental Research Laboratory, Cincinnati, Ohio.

Redding, J., et al. 1975. Fuel Consumption: Fuel Utilization and Conservation in Industry. Document No. PB-246-999. Down Chemical, Freeport, Texas.

Reding, J. T., et al. 1975. Energy Consumption: The Chemical Industry. EPA 650/2-75-032a, PB 241 927, Environmental Protection Agency, washington, D.C.

Sapp, J. E. 1981. Energy and Materials Flows in the Cement Industry. ANL/CNSV-17, Argonne National Laboratory, Argonne, Illinois. 
Schorr, J. R. and G. A. Anderson. 1975. Industrial Energy Study of the Glass Industry. Battelle Columbus Laboratories, Columbus, Ohio.

Schwartzkopf, Florian. 1970. "A Comparison of Modern Lime Calcining Systems." Rock Products. Maclean Hunter Publishing Corporation, Chicago, Illinois.

Shen, S. Y., et al. 1980. Energy and Materials Flows in the Production of Liquid and Gaseous 0xygen. ANL/CNSV-15, Argonne National Laboratory, Argonne, Ilínois.

Shreve, R. N. and J. A. Brink, Jr. 1977. Chemical Process Industries. McGraw-Hill Book Company, New York, New York.

Sittig, M. 1977. Particulates and Fine Dust Removal: Equipment and Control Practices. Noyes Data Corporation, Park Ridge, New Jersey.

Sittig, M. 1977. Practical Techniques for Saving Energy in the Chemical, Petroleum, and MetaTs Industries. Noyes Data Corporation, Park Ridge, New Jersey.

Sittig, M. 1977. Pulp and Paper Manufacture Energy Conservation and Pollution Prevention. Noyes Data Corp., Park Ridge, New Jersey.

Streb, A. J. 1977. Final Report for Priority Listing of Industrial Processes by Total Energy Consumption and Potential for Savings. Zonex Corp., Battimore, Maryland.

TAPPI Press. 1976. Introduction to Papermaking Technology, Atlanta, Georgia. Tarrillion, T. B. 1982. "Ethylene: Feedstocks and Their Impact on ByProducts." Chemical Engineering. 89(26):30-32.

United Technologies Corporation. January 1980. Cogeneration Technology Alternatives Study (CTAS), United Technologies Corporation Final Report, Volume II - Industrial Process Characteristics. D0E/NASA/0030-80/2. South Windsor, Connecticut.

Unzelman, George H. and Norman H. Gerber. October 1965. "Hydrocracking Today and Tomorrow". Petro/Chem Engineer.

U.S. Department of Commerce. 1982. Annual Survey of Manufactures: Fuels and Electric Energy Consumed. Bureau of Census, Washington, D.C.

U.S. Department of Energy. 1977. "Workshop on Energy Conservation in the Pulp and Paper Industry." U.S. Department of Energy, Washington, D.C.

U. S. Department of Health, Education, and Welfare. 1960. Atmospheric Emissions from Petroleum Refineries - A Guide for Measurement and Control. Public Health Service Publication No. 763. U.S. Government Printing Office, Washington, D.C. 
U.S. Tarriff Commission. 1975. Synthetic Organic Chemicals.

Vogt, C. 1974. Development Document for Effluent Limitations Guidelines and New Source Performance Standards for the Unbleached Kraft and Semichemical Pulp Segment of the Pulp, Paper and Paperboard Mills Point Source Category, EPA440/1-74-025-a, National Technical Information Service, Springfield, Virginia.

Warren, K. and H. S. Leff. 1980. "Stone, Clay and Glass Products." Industrial Energy Use Data Book. ORAU-160, Oak Ridge Associated Universities, Oak Ridge, Tennessee.

Webb, R. L. and A. K. Kulkarni. 1982. Heat Exchanger Needs for Recovering Waste Heat in the Glass Making Industry. DOE/ID/12225-T1, prepared for the U.S. Department of Energy, Washington, D.C.

Wei, G. G., et al. 1981. "Brayton Cycle Heat Recovery for Container Glass Melting Furnace." Proceedings of the 16th Intersociety Energy Conversion Engineering Conference, ASME, New York, New York.

"1982 Refining Process Handbook". September 1982. Hydrocarbon Processing. 
. 
PNL -4912

UC $-95 f$

DISTR IBUTION

No. of

Copies

OFFSITE

William B. Williams

U.S. Department of Energy

Office of Industrial Programs

Forrestal Building

Washington, D.C. 20585

John Rossmeissl

U.S. Department of Energy

Office of Industrial Programs

Forrestal Building

Washington, D.C. 20585

Al Hayes

U.S. Department of Energy

Office of Industrial Programs

Forrestal Building

Washington, D.C. 20585

\author{
Al Streb \\ Deputy Assistant Secretary for \\ Conservation
}

U.S. Department of Energy

Forrestal Building

Washington, D.C.

W. H. Thielbahr, Manager

Energy Conservation Branch

Energy \& Technology Division

U.S. Department of Energy

Idaho Operations Office

550 Second Street

Idaho Falls, Idaho 83401

27 DOE Technical Information Center
No. of

Copies

ONSITE

DOE Richland Operations Office

H. E. Ransom/D. R. Segna

42 Pacific Northwest Laboratory

C. H. Bloomster

D. R. Brown

R. E. Dodge

L. L. Fassbender

R. M. Fleischman

B. A. Garrett Price

E. A. Griffin

H. D. Huber

N. L. Moore (15)

S. A. Smith

R. L. Watts

G. L. Wilfert (10)

Publishing Coordination (2)

Technical Information (5) 
